\title{
SEARCH FOR THE STANDARD MODEL HIGGS BOSON IN THE MISSING TRANSVERSE ENERGY AND B-JET SIGNATURE IN PROTON-ANTIPROTON COLLISIONS AT A CENTER OF MASS ENERGY OF $1.96 \mathrm{TEV}$
}

\author{
A Dissertation \\ Submitted to the Faculty \\ of \\ Purdue University \\ by \\ Artur Apresyan
}

\author{
In Partial Fulfillment of the \\ Requirements for the Degree \\ of \\ Doctor of Philosophy
}

May 2009

Purdue University

West Lafayette, Indiana 
To my parents, Henrik and Iskuhi, my wife Paytsar and daughter Ani. 


\section{ACKNOWLEDGMENTS}

While working on this dissertation, I often imagined of the moment when I would get to this part. I now look back at the last six years spent at Purdue with pleasure, nostalgia and gratitude. There are many people I would like to thank, without whose contributions this work would have been impossible. I am glad to have an opportunity to remember wonderful people who have contributed to this thesis in direct or indirect way.

My utmost gratitude goes to my adviser, Daniela Bortoletto, for her unlimited guidance and support throughout my graduate studies at Purdue, and for the love of physics that she radiates. She taught me how to be a researcher, starting from a thorough understanding of the problem and working persistently to achieve the solution. I have been fortunate to have an adviser that gave me the freedom to explore ideas on my own, and at the same time gave me the encouragement and invaluable advice when I was confused and uncertain. I hope that at some point I can be as good an adviser to my students as Daniela has been to me.

I would like to express a deep gratitude to Gino Bolla and Petra Merkel. Over the years that we know each other, you have constantly supported me both professionally and personally, and I can never thank you enough for that. I hope that I was able to learn from your work attitude and efficiency during the time I spent with you in the Silicon Lab at Purdue. You showed me what the right approaches are, both in work and in life outside of it.

It is my pleasure to thank everybody directly involved in this analysis, for their great help and advice. I would like to thank Viktor Veszpremi for his help when I was starting my work at CDF, for countless useful advices and laying out the foundations of the MET+Jets analysis. Oscar Gonzalez and Song Ming Wang for their physics insights and numerous opportunities they gave me to learn from discussion with them. 
I would like to thank Karolos Potamianos for his help in this analysis and for making me a big fan of Belgian chocolate.

Special thanks goes to Fabrizio Margaroli, for his enormous support and guidance during this work. Our discussions about physics and unrelated topics have had the greatest impact on me, and I would like to think that I became a better physicist and person as a result. Working on this analysis without your "fountain" of new ideas, enthusiasm and inspiration would have been a lot harder, and less enjoyable. You always encouraged me by praising my work and always preserved confidence in my ability to achieve the goal.

I would like to thank the people who inspired, supported and helped me towards this work in many different ways, they all gave me an abundance of lasting memories. Iuri Oksuzian and Sergo Jindariani for your encouragement and fun discussions we had in the CDF outback. Diego Tonelli and Nathan Goldschmidt for numerous thoughtful advices you have given, making difficult situations appear much less challenging. Federica Moscato for countless discussions, which at times transformed into passionate arguments, but have dramatically changed my views on many things. I would like to thank Nathan, Diego, Karolos, Fabrizio and Federica for helping me to formulate the content of a challenging proposal, it was certainly one of the most fun moments during my stay at CDF. Taisuke Minagawa for being a true friend and sharing all of the ups and downs, and for the all-nighters we spent together trying to finish the assignments in time. Edwin Antillon, with whom I have shared many ideas and laughs, I will most certainly miss the conversations with you.

Vladimir Solovyev, Valeria Starovoitova and Vassili Maroussov, you made my stay at Purdue appear much closer to home. Thank you Valeria for your priceless friendship and most enjoyable parties. Arshak Petrosyan was like a big brother to me and was always there to help whenever I encountered a problem. Arshak, Karen and Liana Hayrapetyan, you became my family in the US and helped me keep my culture alive while being away from home. 
I am deeply indebted to my family for their continued support and unwavering faith in me throughout my academic career and life. Dear Mom and Dad, thank you for giving me a solid foundation and for your active presence in my life. You have always accepted my dreams even if they were different from yours, and did your best in helping me realize them. My sister Karine for the support and care that you gave me. I want to especially thank my wife, Paytsar for her unlimited support throughout my life, and the sacrifices that she selflessly made to help me advance. I acknowledge a debt and appreciation which extends beyond any words at my command. Without your love, encouragement, and help I couldn't have finished my thesis. I would also like to thank my daughter, Ani, for giving me unlimited happiness, pleasure and a completely new meaning to my life, and for patiently waiting for me to play with her. 


\section{TABLE OF CONTENTS}

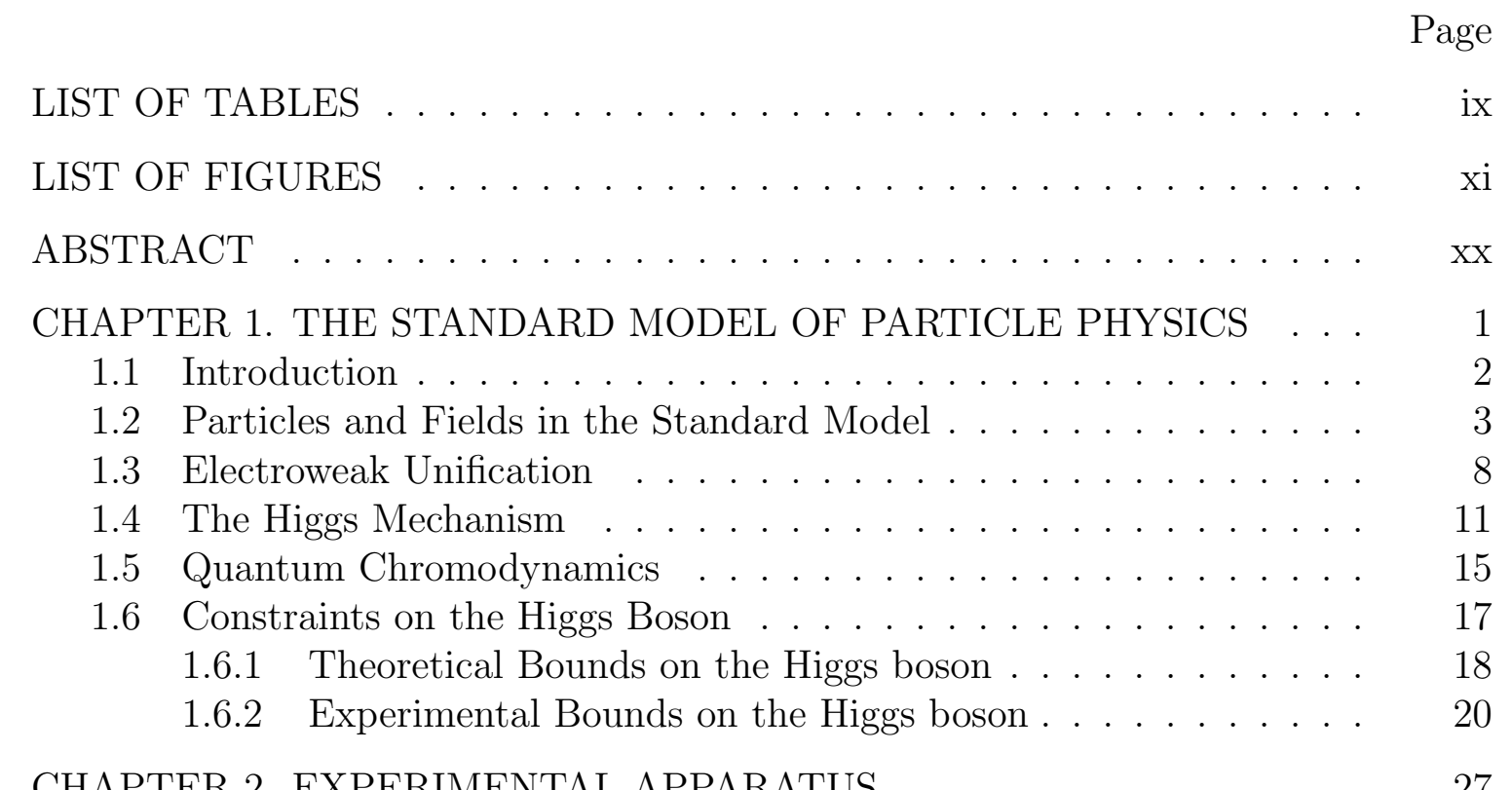

2.1 Accelerator Complex . . . . . . . . . . . . . . . . 27

2.1.1 The Linear Accelerator and Booster . . . . . . . . . . . 29

2.1.2 The Antiproton Source . . . . . . . . . . . . . . . . . . 30

2.1 .3 The Recycler Ring . . . . . . . . . . . . . . . . . . 31

2.1 .4 The Main Injector . . . . . . . . . . . . . . . . . 31

2.1 .5 The Tevatron . . . . . . . . . . . . . . . . . . 32

2.2 The CDF II detector . . . . . . . . . . . . . . . . . . . . . . . 32

2.2 .1 Tracking System . . . . . . . . . . . . . . . 34

2.2 .2 Central Outer Tracker . . . . . . . . . . . . . . 36

2.2 .3 Time of Flight Detector . . . . . . . . . . . . . . . . . 38

2.2 .4 Superconducting Solenoid . . . . . . . . . . . . . . . 39

2.2 .5 Calorimetry System . . . . . . . . . . . . . . . . . . . 39

2.2 .6 Muon Detector . . . . . . . . . . . . . . . . . . 43

2.3 Luminosity Counting . . . . . . . . . . . . . . . . . 45

2.4 Trigger and Data Acquisition . . . . . . . . . . . . 45

2.4 .1 Level 1 Trigger . . . . . . . . . . . . . . . . . . . . 48

2.4 .2 Level 2 Trigger . . . . . . . . . . . . . . . . . . . . 49

2.4.3 Level 3 Trigger . . . . . . . . . . . . . . . . . . . . . 49

CHAPTER 3. EVENT RECONSTRUCTION . . . . . . . . . . . . 50

3.1 Event Kinematics . . . . . . . . . . . . . . . . . . . . . 50 
3.2 Data Format and Analysis Software . . . . . . . . . . . . 51

3.3 Track Reconstruction . . . . . . . . . . . . . . . . 51

3.4 Primary Vertex Reconstruction . . . . . . . . . . . . . 53

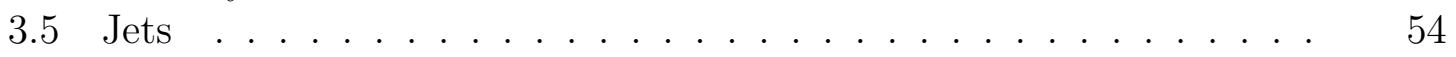

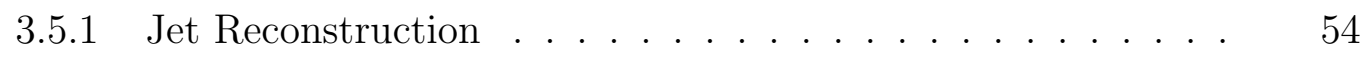

3.5.2 Generic Jet Energy Corrections . . . . . . . . . . . . . 56

3.5.3 Jet Energy Corrections using H1 algorithm . . . . . . . . . . 58

3.6 Missing Transverse Energy: $\mathbb{t}_{T} \ldots \ldots \ldots \ldots 1$

3.6.1 $\mathbb{E}_{T}$ Corrections . . . . . . . . . . . . . 63

3.6.2 Missing Transverse Momentum: $\not T_{T}^{t r} \ldots \ldots . . . . . . .663$

3.7 Identification of $b$-quark Jets: $b$-tagging . . . . . . . . . . . 66

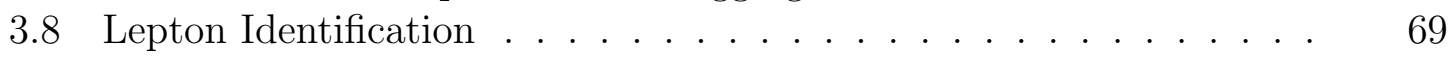

3.8.1 Electron Reconstruction . . . . . . . . . . . . 69

3.8.2 Isolated Tracks . . . . . . . . . . . . . . . 71

CHAPTER 4. TRIGGER PATH AND EVENT PRESELECTION . . . . . 72

$4.1 \notin_{T}$ Triggers at CDF . . . . . . . . . . . . . . . . 73

4.1.1 MET35_\&_TWO_JETS Trigger . . . . . . . . . . . . 74

4.1.2 Level $1 \notin_{T}$ Reconstruction . . . . . . . . . . . . . . . . . . . 75

4.1.3 Level 2 Jet Reconstruction . . . . . . . . . . . . . . . . . 76

4.1.4 Level $3 \notin_{T}$ Reconstruction . . . . . . . . . . . . . . . . 76

4.2 Event Preselection . . . . . . . . . . . . . . . . . 76

4.2.1 Quality selections for $\mathbb{E}_{T}$ analysis ............ 76

4.2.2 Run Selection . . . . . . . . . . . . . . . . . . 78

4.2.3 Trigger Efficiency . . . . . . . . . . . . . . . . . 79

4.2.4 Event Selection for the Analysis Sample . . . . . . . . . 84

CHAPTER 5. SIGNAL AND BACKGROUND MODELING . . . . . . . . . 88

5.1 Signal Modeling . . . . . . . . . . . . . . . . . . . . 88

5.2 Background Modeling . . . . . . . . . . . . . . . . . . . 89

5.2.1 Multijet Production . . . . . . . . . . . . . . . . . . 90

5.2.2 Di-boson and $W / Z$ backgrounds . . . . . . . . . . . . . 100

5.2 .3 Top Production . . . . . . . . . . . . . . 100

CHAPTER 6. CONTROL REGIONS AND SIGNAL BOX . . . . . . . . . . 102

6.1 Definitions . . . . . . . . . . . . . . . 103

6.2 Background Model Comparison with Data in Control Regions . . . 104

CHAPTER 7. SIGNAL REGION OPTIMIZATION . . . . . . . . . . . . 115

7.1 Statistical Learning Methods . . . . . . . . . . . . . . . 115

7.1.1 Artificial Neural Networks . . . . . . . . . . . . . . . 117

7.2 Multijet Rejection Neural Network . . . . . . . . . . . . . . . 119

7.3 QCD Enriched Control Region 3 . . . . . . . . . . . . . 129

7.4 Normalization of the Multijet Background . . . . . . . . . . . . 134 
7.5 Signal Region Definition . . . . . . . . . . . . . . . 135

CHAPTER 8. SEARCH FOR THE HIGGS BOSON . . . . . . . . . . . . . 136

8.1 Discriminant Neural Network . . . . . . . . . . . . . 136

8.2 Systematic Uncertainties . . . . . . . . . . . . . . . . . . 140

8.3 Measurement in the Signal Region . . . . . . . . . . . . . . . . . . 147

8.4 Limit Calculation . . . . . . . . . . . . . . . . . . . 153

8.5 Results . . . . . . . . . . . . . . . . 156

8.6 Conclusion . . . . . . . . . . . . . . . . . . . 159

LIST OF REFERENCES . . . . . . . . . . . . . . . . . . . . . . 164

APPENDICES

Appendix A: Missing $E_{T}$ and Additional Triggers . . . . . . . . . . . . 170

Appendix B: Additional Distributions in Control Regions . . . . . . . . . 173

Appendix C: Correlations in Control Regions . . . . . . . . . . . . . . . 201

Appendix D: Additional Plots for ANNs . . . . . . . . . . . . 208

VITA . . . . . . . . . . . . . . . . . . . 213 


\section{LIST OF TABLES}

Table

Page

1.1 Properties of elementary particles in the Standard Model . . . . . . . .

1.2 Masses of the $W$ and $Z$ bosons as measured by UA1 and UA2 experiments, and their most precise current values [10]. . . . . . . . . 14

1.3 The Higgs boson couplings to fermions and bosons . . . . . . . . . . . 18

2.1 Characteristic parameters of the Tevatron accelerator . . . . . . . . . 29

2.2 Main characteristics of the EM and HAD calorimeters of CDF. . . . . . 41

3.1 Quality requirements for tracks used in $\not P_{T}^{t r}$ calculation. The rows are listed in the order the selections are checked. If the first row requirements fail, we next check the second row, etc. . . . . . . . . . . . . . 65

3.2 Electron identification selections using central and plug calorimeters. . 70

3.3 Summary of isolated track identification selections. . . . . . . . . . . 71

4.1 Contributions to $2 / 3$ jet events from different decay modes of the $W$-boson in $W H$ events . . . . . . . . . . . . . . . . . . . . . . . . . . . . . . . . 85

5.1 The cross-section times branching ratios (number of events produced per $\mathrm{fb}^{-1}$ ) of the $Z H / W H$ processes at various Higgs mass hypothesis . . .

5.2 The binning used for the ST+ST Tag Rate Matrix _ . . . . . . . . . . 94

5.3 The binning used for the Exclusive ST Tag Rate Matrix . . . . . . . . 99

6.1 Number of expected and observed events in control regions in Exclusive ST events. The expected Higgs boson signal is shown for $m_{H}=115 \mathrm{GeV} / c^{2}$. The uncertainties contain both Monte Carlo statistical error and systematic uncertainties. . . . . . . . . . . . . . .

6.2 Number of expected and observed events in control regions in $\mathrm{ST}+\mathrm{ST}$ events. The expected Higgs boson signal is shown for $m_{H}=115 \mathrm{GeV} / c^{2}$. The uncertainties contain both Monte Carlo statistical error and systematic uncertainties. . . . . . . . . . . . . . . . . .

6.3 Number of expected and observed events in control regions in ST+JP events. The expected Higgs boson signal is shown for $m_{H}=115 \mathrm{GeV} / c^{2}$. The uncertainties contain both Monte Carlo statistical error and systematic uncertainties. . . . . . . . . . . . . . . . . 
Table

7.1 Rejection rates after $\mathrm{ANN}_{\mathrm{MJ}}>0$ selection for signal, all backgrounds and QCD MC only. . . . . . . . . . . . . . . . . . . . .

7.2 Acceptance of signal and backgrounds after selection criteria in $2.1 \mathrm{fb}^{-1}$. The events were weighted by cross-section and efficiencies after applying the selections. . . . . . . . . . . . . . . . . .

7.3 Number of expected and observed events in CR3. The expected Higgs boson signal is shown for $m_{H}=115 \mathrm{GeV} / c^{2}$. The uncertainties contain both Monte Carlo statistical error and systematic uncertainties. . . .

7.4 Multijet background scale factors in different control regions, for different tagging categories. . . . . . . . . . . . . . . .

8.1 Uncertainties due to trigger efficiency . . . . . . . . . . . . . . . 143

8.2 ISR/FSR uncertainties . . . . . . . . . . . . . . . . . . . 144

8.3 Summary of systematic uncertainties in the Exclusive ST sample . . . 145

8.4 Summary of systematic uncertainties in the ST+ST sample . . . . . . . 145

8.5 Summary of systematic uncertainties in the ST+JP sample . . . . . . . 146

8.6 Comparison of the total number of expected and observed events in the signal region for different $b$-tagging categories. The uncertainties contain both statistical and systematic uncertainties. The expected signal is shown

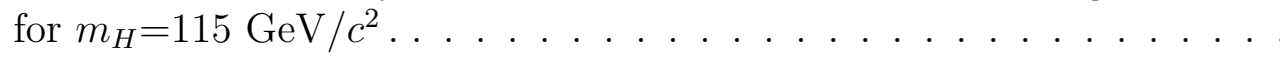

8.7 The combined $95 \%$ C.L. upper limits on cross section time $H \rightarrow b \bar{b}$ branching fraction when the Higgs boson is produced in association with weak boson. The last two columns give the ratio of the expected and observed limits with respect to the SM cross section. . . . . . . . . . .

D.1 Variables used in Fig. D.2, as they appear from top to bottom (left to right) on the correlation comparison plots. . . . . . . . . . . . .

D.2 Variables used in Fig. D.3-D.4, as they appear from top to bottom (left to right) on the correlation comparison plots. . . . . . . . . . . . . 


\section{LIST OF FIGURES}

Figure

Page

$1.1 e^{+} e^{-} \rightarrow \mu^{+} \mu^{-}$scattering at the leading order (left), and with one loop (right) . . . . . . . . . . . . . . . .

1.2 Feynman diagrams for (a) strong, (b) electromagnetic and (c) weak interactions. $Q^{2}$ is the square of 4 -momentum transferred between the particles. . . . . . . . . . . . . . . . .

1.3 Perturbative and stability bounds on Higgs boson mass as a function of the Planck Scale $\Lambda[28]$. . . . . . . . . . . . . . . . . .

1.4 $\Delta \chi^{2}$ of the global fit to the Standard Model as a function of the Higgs boson mass [32] . . . . . . . . . . . . . . . . . . . . 21

1.5 Constraints on Higgs Mass from latest Tevatron measurement [33] . . . 22

1.6 Standard Model Higgs boson at the Tevatron . . . . . . . . . . . . . 23

2.1 A schematic view of the Teavtron . . . . . . . . . . . . . . . 28

2.2 A cross-sectional view of one half of the CDF II detector. . . . . . . . . 33

2.3 A view of the CDF tracking system: (a) cross section view of the CDF II tracking system, (b) the $r-z$ view of the CDF II silicon system . . . .

2.4 The L00 detector: (a) a schematic overview of the L00 detector and (b) the impact parameter resolution of tracks with L00 hits (red) and without L00 hits (blue) . . . . . . . . . . . . . . .

2.5 The SVX II detector: (a) the view of the three SVXII barrels, (b) a schematic overview of the SVX components . . . . . . . . . .

2.6 The ISL detector: (a) a perspective view of the ISL space frame and silicon placement, (b) a schematic overview of the ISL components . . . . .

2.7 The COT detector: (a) 1/6 section of the COT end plate. For each superlayer the total number of supercells, the wire orientation (axial or stereo), and the average radius is given. (b) Three supercells looking along the beam $z$ direction. . . . . . . . . . . . . . .

2.8 Mass distribution from TOF measurement versus momentum for positive and negative tracks. The three horizontal lines correspond to nominal $p$, $K$, and $\pi$ masses. . . . . . . . . . . . . . . . . . . . 
2.9 (a) elevation view of one half of the CDF detector displaying the components of the CDF calorimeter, (b) the central calorimetry wedges and the location of the preshower and crack detectors. . . . . . . . . . .

2.10 Muon detectors in CDF: (a) map of the muon coverage as a function of azimuthal angle $\phi$ and pseudorapidity $\eta$, (b) transverse view of the CMU, showing the four layers in the radial direction . . . . . . . . . .

2.11 Cherekov luminosity monitors in CDF: (a) the CLC module shown inside the CDF end-plug calorimeters, (b) schematic view of the luminosity monitor inside a quadrant of $\mathrm{CDF}$

2.12 The CDF trigger and data acquisition system. Typical trigger rates and rejection factors for the three-level system are shown. . . . . . . . . . 47

2.13 The Run II trigger-system block diagram. . . . . . . . . . . . . . . . . 48

3.1 Track of a positively charged particle. . . . . . . . . . . . . . . 52

3.2 Total systematic uncertainty on the jet energy scale. . . . . . . . . . 58

3.3 Performance of the $\mathrm{H} 1$ corrections compared to the generic jet energy corrections [65]: (a) comparison of the jet energy resolution for jets in $0.1<|\eta|<0.7$ after generic and H1 corrections, (b) comparison of the average uncertainty on the jet energy scale. . . . . . . . . . .

3.4 Tagging efficiencies for the SecVtx algorithm as a function of jet (a) $E_{T}$

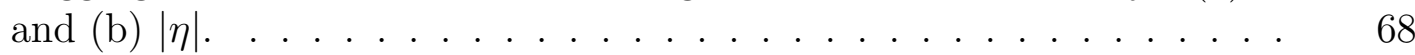

3.5 Tagging efficiencies for the JetProb algorithm as a function of jet $E_{T}$. . 68

4.1 Efficiency of the L1 MET25 trigger in $2.1 \mathrm{fb}^{-1}$ for (a) CMUP18, (b) JET20, and (c) JET50. Parametrization of the trigger efficiency is shown in red, as a function of corrected $\mathbb{E}_{T} \ldots \ldots \ldots \ldots$

4.2 Efficiency of the L3 MET35 trigger in $2.1 \mathrm{fb}^{-1}$ for (a) CMUP18, (b) JET20, and (c) JET50. Parametrization of the trigger efficiency is shown in red, as a function of corrected $\mathbb{E}_{T} \ldots \ldots \ldots \ldots$. . . . . . . . .

4.3 Comparison of total trigger efficiency of the MET+Jets sample, obtained from several samples. The lower plot shows the relative difference from the nominal values. The band indicates the relative uncertainty. . . . .

5.1 The distribution of $\Delta \phi\left(\overrightarrow{\mathscr{E}}_{T}, \vec{J}_{2}\right)$ in MET+Jets sample compared to $Z H$ Monte Carlo simulation (the scale is arbitrary). . . . . . . . . . 
5.2 The definitions of the control regions and the TRM region. The line separating the Signal Box and CR2 is for illustrative purpose only. The only difference between these two regions is that we require at lease one charged lepton in CR2, while in Signal Box all events with charged leptons are vetoed. . . . . . . . . . . . . . . . . . . . .

5.3 The distributions of jet fragmentation function $Z$ for jets in QCD Monte Carlo simulation (the scale is arbitrary). The red distribution correspond to $b$-quark jets, the green curve to $c$-quark jets and the black curve to light flavor jets. . . . . . . . . . . . . . . . . .

5.4 Tagging probabilities for ST+ST events, as a function of TRM variables: (a) jet $E_{T}$, (b) jet $\eta$, (c) event $H_{T}$, (d) jet $Z$. The distributions are shown using the same bin sizes as in Tab. $5.2 \ldots \ldots$. . . . . . . .

5.5 Tagging probabilities for ST+JP events, as a function of TRM variables: (a) jet $E_{T}$, (b) jet $\eta$, (c) event $H_{T}$, (d) jet $Z$. The distributions are shown using the same bin sizes as in Tab. $5.2 \ldots \ldots$. . . . . . . .

5.6 Tagging probabilities for Exclusive ST events, as a function of TRM variables: (a) jet $E_{T}$, (b) jet $\eta$, (c) event $H_{T}$, (d) jet $Z$. The distributions are shown using the same bin sizes as in Tab. 5.2 . . . . . . . . . . .

6.1 Kinematic distributions in QCD CR1 for events with single b-tag: (a) dijet invariant mass, (b) event $\not_{T}$, (c) $\Delta \phi\left(\vec{\not}_{T}^{t r}, \vec{\notin}_{T}\right)$, (d) event $\not P_{T}^{t r}$, (e) maximum of the difference in $\phi$ between two jets directions, (f) transverse energy of the $3^{r d}$ jet . . . . . . . . . . . . . . . .

6.2 Kinematic distributions in CR2 for events with single b-tag: (a ) dijet invariant mass, (b) invariant mass of $\mathbb{E}_{T}$ and all jets, (c) number of jets, (d) $\Delta \phi\left(\vec{\not}_{T}^{t r}, \vec{E}_{T}\right)$, (e) minimum of the difference in $\phi$ between the missing transverse energy and each jet, (f) transverse energy of the $3^{\text {rd }}$ jet . . .

6.3 Kinematic distributions in QCD CR1 for ST+ST events: (a) dijet invariant mass, (b) event $\not_{T}$, (c) $\Delta \phi\left(\vec{\not}_{T}^{t r}, \vec{\notin}_{T}\right)$, (d) event $\not_{T}^{t r}$, (e) maximum of the difference in $\phi$ between two jets directions, (f) transverse energy of the $3^{r d}$ jet . . . . . . . . . . . . . . . . . .

6.4 Kinematic distributions in CR2 for ST+ST events: (a ) dijet invariant mass, (b) invariant mass of $\mathscr{E}_{T}$ and all jets, (c) number of jets, (d) $\Delta \phi\left(\vec{\phi}_{T}^{t r}, \vec{\notin}_{T}\right)$, (e) minimum of the difference in $\phi$ between the missing transverse energy and each jet, (f) transverse energy of the $3^{\text {rd }}$ jet . . . . . . . . . 
Figure

6.5 Kinematic distributions in QCD CR1 for ST+JP events: (a) dijet invariant mass, (b) event $\not_{T}$, (c) $\Delta \phi\left(\vec{\not}_{T}^{t r}, \vec{\notin}_{T}\right)$, (d) event $\not_{T}^{t r}$, (e) maximum of the difference in $\phi$ between two jets directions, (f) transverse energy of the $3^{\text {rd }}$

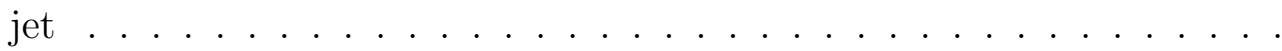

6.6 Kinematic distributions in CR2 for ST+JP events: (a ) dijet invariant mass, (b) invariant mass of $\mathscr{E}_{T}$ and all jets, (c) number of jets, (d) $\Delta \phi\left(\vec{\not}_{T}^{t r}, \vec{E}_{T}\right)$, (e) minimum of the difference in $\phi$ between the missing transverse energy and each jet, (f) transverse energy of the $3^{\text {rd }}$ jet . . . . . . . . . . .

7.1 The training of a statistical learning method, that builds a model (classifier) which is then used to evaluate new events. . . . . . . . . . .

7.2 Architecture of a one-hidden-layer feed-forward ANN [88].

7.3 Kinematic distributions of background and signal samples used in the $\mathrm{ANN}_{\mathrm{MJ}}$ training. The distributions are normalised to an arbitrary area. The signal is shown in solid histogram, and the QCD background in hatched. . . . . . . . . . . . . . . . . .

7.4 Multijet rejection ANN performance: (a) $\mathrm{ANN}_{\mathrm{MJ}}$ output for the testing and training samples, (b) the convergence test of $\mathrm{ANN}_{\mathrm{MJ}}$. . . . . . .

7.5 Signal acceptance versus background rejection plot, scanning over values of $\mathrm{ANN}_{\mathrm{MJ}}$. The dotted line shows that using the $\mathrm{ANN}_{\mathrm{MJ}}$, we can keep the same background rejection as in the cut-based selection while increasing the signal acceptance by $\sim 10 \%$. . . . . . . . . . . . . . .

7.6 The distribution of $\mathrm{ANN}_{\mathrm{MJ}}$ in control regions: Exclusive $\mathrm{ST}$ events in (a) CR1 and (b) CR2; ST+ST events in (c) CR1 and (d) CR2; ST+JP events in $(\mathrm{e}) \mathrm{CR} 1$ and $(\mathrm{f}) \mathrm{CR} 2 \ldots \ldots \ldots$. . . . . . . . . . . . .

7.7 The distribution of $\mathrm{ANN}_{\mathrm{MJ}}$ before applying the final selection of $\mathrm{ANN}_{\mathrm{MJ}}>$ 0: events in (a) exclusive ET and (b) ST+ST and (c) ST+JP . . . . .

7.8 Kinematic distributions in CR3 for events with single b-tag: (a) dijet invariant mass, (b) event $\not_{T}$, (c) $\Delta \phi\left(\vec{\not}_{T}^{t r}, \vec{\not}_{T}\right)$, (d) event $\not_{T}^{t r}$, (e) maximum of the difference in $\phi$ between two jets directions, (f) transverse energy of the $3^{r d}$ jet . . . . . . . . . . . . . . . . .

7.9 Kinematic distributions in CR3 for ST+ST events: (a) dijet invariant mass, (b) event $\not_{T}$, (c) $\Delta \phi\left(\vec{\not}_{T}^{t r}, \vec{E}_{T}\right)$, (d) event $\not_{T}^{t r}$, (e) maximum of the difference in $\phi$ between two jets directions, (f) transverse energy of the $3^{\text {rd }}$

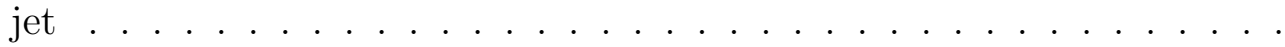


7.10 Kinematic distributions in QCD CR1 for ST+JP events: (a) dijet invariant mass, (b) event $\notin_{T}$, (c) $\Delta \phi\left(\vec{\phi}_{T}^{t r}, \vec{E}_{T}\right)$, (d) event $\not P_{T}^{t r}$, (e) maximum of the difference in $\phi$ between two jets directions, (f) transverse energy of the $3^{\text {rd }}$

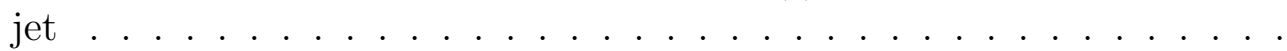

8.1 Kinematic distributions of background and signal samples used in the training of $\mathrm{ANN}_{\mathrm{SIG}}$ for 2 jet events. The distributions are normalized to an arbitrary area. The signal is shown in solid histogram, and the sum of MET+Jets untagged data and $t \bar{t}$ background in hatched. . . . . . . . .

8.2 Performance of $\mathrm{ANN}_{\mathrm{SIG}}$ for events with 2 jets: (a) $\mathrm{ANN}_{\mathrm{SIG}}$ output for the testing and training samples, (b) the convergence test of $\mathrm{ANN}_{\mathrm{SIG}}$. . . .

8.3 Kinematic distributions of background and signal samples used in the training for 3 jet events. The distributions are normalised to an arbitrary area. The signal is shown in solid histogram, and the sum of MET+Jets untagged data and $t \bar{t}$ background in hatched.

8.4 Performance of $\mathrm{ANN}_{\mathrm{SIG}}$ for events with 3 jets: (a) $\mathrm{ANN}_{\mathrm{SIG}}$ output for the testing and training samples, (b) the convergence test of $\mathrm{ANN}_{\mathrm{SIG}}$. A spike of signal events observed in the background-like region of $\mathrm{ANN}_{\mathrm{SIG}}$ output $(-0.8,-0.6)$ is due to events where one of jets from the Higgs boson is detected as the third leading jet. This results in the wrong value of $M_{j j}$, which is constructed from the two leading jets. . . . . . . . . .

8.5 Kinematic distributions of the inputs to the $\mathrm{ANN}_{\mathrm{SIG}}$ in the signal region for Exclusive ST events: (a) dijet invariant mass, (b) invariant mass of all jets and $\not_{T}$, (c) event $H_{T}-\notin_{T}$, (d) event $\not H_{T}-\not_{T}$, (e) ANN trackMET, (f) maximum $\Delta R$ between all jets. The distributions for the $m_{H}=115 \mathrm{GeV} / c^{2}$ Higgs boson signal are shown in the overlaid black histogram (scaled by a factor of 25).

8.6 Kinematic distributions of the inputs to the $\mathrm{ANN}_{\mathrm{SIG}}$ in the signal region for ST+ST events: (a) dijet invariant mass, (b) invariant mass of all jets and

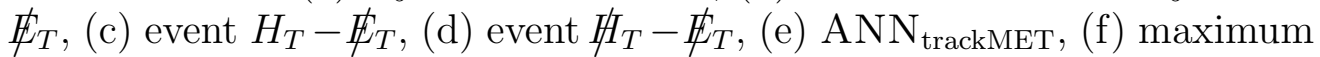
$\Delta R$ between all jets. The distributions for the $m_{H}=115 \mathrm{GeV} / c^{2}$ Higgs boson signal are shown in the overlaid black histogram (scaled by a factor of 5$) \ldots \ldots \ldots \ldots \ldots \ldots \ldots \ldots \ldots \ldots \ldots \ldots$

8.7 Kinematic distributions of the inputs to the $\mathrm{ANN}_{\mathrm{SIG}}$ in the signal region for ST+JP events: (a) dijet invariant mass, (b) invariant mass of all jets and

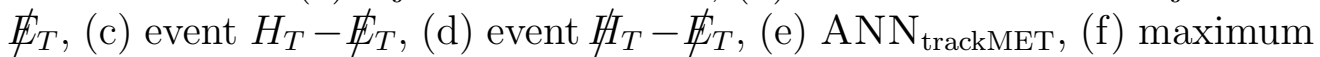
$\Delta R$ between all jets. The distributions for the $m_{H}=115 \mathrm{GeV} / c^{2}$ Higgs boson signal are shown in the overlaid black histogram (scaled by a factor of 5$) \ldots \ldots \ldots \ldots \ldots \ldots \ldots \ldots \ldots \ldots \ldots$ 
Figure

8.8 $\mathrm{ANN}_{\mathrm{SIG}}$ distributions in the signal region. The distributions for the $m_{H}=$ $115 \mathrm{GeV} / c^{2}$ Higgs boson signal are shown in an overlaid black histogram (scaled by a factor of 25 for single-tagged events and a factor of 5 for double-tagged events). . . . . . . . . . . . . . .

8.9 Expected and observed limits as a ratio compared to Standard Model cross sections. Black line represents the $R_{\text {obs }}$ values and the red dotted line shows the $R_{\text {exp }}$ values at different masses . . . . . . . . . . .

8.10 The Tevatron combined 95\% C.L. upper limit as a function of the Higgs boson mass between 100 and $200 \mathrm{GeV} / c^{2}$ Solid black: observed limit/SM; Dashed black: median expected limit/SM. Colored bands: $\pm 1,2 \sigma$ distributions around median expected limit. . . . . . . . . . . . . . .

8.11 The CDF combined 95\% C.L. upper limit as a function of the Higgs boson mass between 100 and $200 \mathrm{GeV} / c^{2}$. Solid lines indicate the observed upper limit for each channel and combined result (dark red). Dashed lines indicate the median expected upper limit for each channel and the combined result (dark red).

8.12 The projected sensitivity of CDF searches for the Higgs boson (a) for $m_{H}=115 \mathrm{GeV} / c^{2}$ and (b) for $m_{H}=160 \mathrm{GeV} / c^{2}$. The combination of $\mathrm{CDF}$ and D0 searches will allow to further increase the sensitivity of the Higgs searches, by doubling the analyzed data. . . . . . . . . . . .

B.1 Kinematic distributions in CR1 for events with single $b$-tag: (a) $1^{\text {st }}$ jet $E_{T}$, (b) $2^{\text {nd }}$ jet $E_{T}$, (c) event $H_{T}$, (d) $1^{\text {st }}$ jet fragmentation function $Z$, (e) $2^{\text {nd }}$ jet fragmentation function $Z$, (f) invariant transverse mass of all jets and $\mathbb{E}_{T} \ldots \ldots \ldots \ldots \ldots \ldots \ldots$

B.2 Kinematic distributions in CR1 for events with single $b$-tag: (a) minimum of the difference in $\phi$ between $\overrightarrow{\not P}_{T}^{t r}$ and each jet, (b) minimum of the difference in $\phi$ between $\vec{\notin}_{T}$ and each jet, (c) maximum of the difference in $R$ space between any two jets, (d) $\not H_{T}-\mathscr{E}_{T}$, (e) $H_{T}-\mathscr{E}_{T}$, (f) $\not_{T} / \mathscr{E}_{T} \ldots$.

B.3 Kinematic distributions in CR1 for events with single b-tag: (a) output

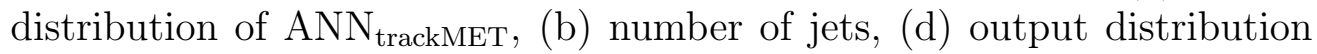

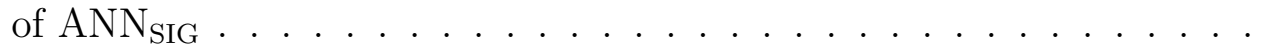

B.4 Kinematic distributions in CR2 for events with single $b$-tag: (a) $1^{\text {st }}$ jet $E_{T}$, (b) $2^{\text {nd }}$ jet $E_{T}$, (c) event $H_{T}$, (d) $1^{\text {st }}$ jet fragmentation function $Z$, (e) $2^{\text {nd }}$ jet fragmentation function $Z,(\mathrm{f})$ event $\mathbb{E}_{T} \ldots \ldots \ldots$ 
B.5 Kinematic distributions in CR2 for events with single b-tag: (a) minimum of the difference in $\phi$ between $\vec{\not}_{T}^{t r}$ and each jet, (b) maximum of the difference in phi between two jets directions, (c) maximum of the difference in $R$ space between any two jets, (d) $\not_{T}-\mathscr{E}_{T}$, (e) $H_{T}-\mathbb{E}_{T}$, (f) $\not H_{T} / \mathbb{E}_{T}$

B.6 Kinematic distributions in CR2 for events with single $b$-tag: (a) output distribution of $\mathrm{ANN}_{\text {trackMET, }}$ (b) event $\not_{T}^{t r}$, (d) output distribution of $\mathrm{ANN}_{\mathrm{SIG}}$

B.7 Kinematic distributions in CR3 for events with single $b$-tag: (a) $1^{\text {st }}$ jet $E_{T}$, (b) $2^{\text {nd }}$ jet $E_{T}$, (c) event $H_{T}$, (d) $1^{\text {st }}$ jet fragmentation function $Z$, (e) $2^{\text {nd }}$ jet fragmentation function $Z$, (f) invariant transverse mass of all jets and

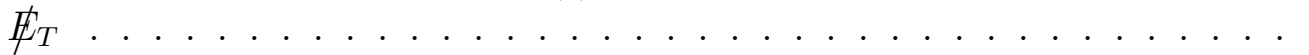

B.8 Kinematic distributions in CR3 for events with single $b$-tag: (a) minimum of the difference in $\phi$ between $\overrightarrow{\not P}_{T}^{t r}$ and each jet, (b) minimum of the difference in $\phi$ between $\vec{\notin}_{T}$ and each jet, (c) maximum of the difference in $R$ space between any two jets, (d) $\not H_{T}-\not_{T}$, (e) $H_{T}-\not_{T}$, (f) $\not H_{T} / \mathscr{E}_{T} \ldots$.

B.9 Kinematic distributions in CR3 for events with single b-tag: (a) output distribution of $\mathrm{ANN}_{\text {trackMET, }}$ (b) number of jets, (d) output distribution of $\mathrm{ANN}_{\mathrm{SIG}} \ldots \ldots \ldots \ldots \ldots$

B.10 Kinematic distributions in CR1 for ST+ST events: (a) $1^{\text {st }}$ jet $E_{T}$, (b) $2^{\text {nd }}$ jet $E_{T}$, (c) event $H_{T}$, (d) $1^{\text {st }}$ jet fragmentation function $Z$, (e) $2^{\text {nd }}$ jet fragmentation function $Z$, (f) invariant transverse mass of all jets and $\not_{T}$

B.11 Kinematic distributions in CR1 for ST+ST events: (a) minimum of the difference in $\phi$ between $\overrightarrow{\not P}_{T}^{t r}$ and each jet, (b) minimum of the difference in $\phi$ between $\vec{E}_{T}$ and each jet, (c) maximum of the difference in $R$ space between any two jets, (d) $\not H_{T}-\mathscr{E}_{T}$, (e) $H_{T}-\mathscr{E}_{T}$, (f) $\not H_{T} / \mathbb{E}_{T} \ldots \ldots$

B.12 Kinematic distributions in CR1 for ST+ST events: (a) output distribution of $\mathrm{ANN}_{\text {trackMET, }}$, (b) number of jets, (d) output distribution of $\mathrm{ANN}_{\mathrm{SIG}}$

B.13 Kinematic distributions in CR2 for ST+ST events: (a) $1^{\text {st }}$ jet $E_{T}$, (b) $2^{\text {nd }}$ jet $E_{T}$, (c) event $H_{T}$, (d) $1^{\text {st }}$ jet fragmentation function $Z$, (e) $2^{\text {nd }}$ jet fragmentation function $Z$, (f) event $\mathbb{E}_{T} \ldots \ldots \ldots$

B.14 Kinematic distributions in CR2 for ST+ST events: (a) minimum of the difference in $\phi$ between $\overrightarrow{\not P}_{T}^{t r}$ and each jet, (b) maximum of the difference in phi between two jets directions, (c) maximum of the difference in $R$ space between any two jets, (d) $\not_{T}-\mathbb{E}_{T}$, (e) $H_{T}-\mathbb{E}_{T}$, (f) $\not_{T} / \mathbb{E}_{T} \ldots \ldots$

B.15 Kinematic distributions in CR2 for ST+ST events: (a) output distribution of $\mathrm{ANN}_{\text {trackMET }}$, (b) event $\not_{T}^{t r}$, (d) output distribution of $\mathrm{ANN}_{\mathrm{SIG}}$. . . 
B.16 Kinematic distributions in CR3 for ST+ST events: (a) $1^{\text {st }}$ jet $E_{T}$, (b) $2^{\text {nd }}$ jet $E_{T}$, (c) event $H_{T}$, (d) $1^{\text {st }}$ jet fragmentation function $Z$, (e) $2^{\text {nd }}$ jet fragmentation function $Z$, (f) invariant transverse mass of all jets and $\mathbb{E}_{T}$

B.17 Kinematic distributions in CR3 for ST+ST events: (a) minimum of the difference in $\phi$ between $\overrightarrow{\not P}_{T}^{t r}$ and each jet, (b) minimum of the difference in $\phi$ between $\vec{E}_{T}$ and each jet, (c) maximum of the difference in $R$ space between any two jets, (d) $\not_{T}-\mathscr{E}_{T}$, (e) $H_{T}-\mathscr{E}_{T}$, (f) $\not H_{T} / \mathbb{E}_{T} \ldots \ldots$

B.18 Kinematic distributions in CR3 for ST+ST events: (a) output distribution of $\mathrm{ANN}_{\text {trackMET, }}$ (b) number of jets, (d) output distribution of $\mathrm{ANN}_{\mathrm{SIG}}$

B.19 Kinematic distributions in CR1 for ST+JP events : (a) $1^{\text {st }}$ jet $E_{T}$, (b) $2^{\text {nd }}$ jet $E_{T},(\mathrm{c})$ event $H_{T}$, (d) $1^{\text {st }}$ jet fragmentation function $Z$, (e) $2^{\text {nd }}$ jet fragmentation function $Z$, (f) invariant transverse mass of all jets and $\mathbb{E}_{T}$

B.20 Kinematic distributions in CR1 for ST+JP events: (a) minimum of the difference in $\phi$ between $\vec{\not}_{T}^{t r}$ and each jet, (b) minimum of the difference in $\phi$ between $\vec{E}_{T}$ and each jet, (c) maximum of the difference in $R$ space between any two jets, (d) $\not_{T}-\mathbb{E}_{T}$, (e) $H_{T}-\mathscr{E}_{T}$, (f) $\not H_{T} / \mathscr{E}_{T} \ldots \ldots$

B.21 Kinematic distributions in CR1 for ST+JP events: (a) output distribution of $\mathrm{ANN}_{\text {trackMET }}$, (b) number of jets, (d) output distribution of $\mathrm{ANN}_{\mathrm{SIG}}$

B.22 Kinematic distributions in CR2 for ST+JP events: (a) $1^{\text {st }}$ jet $E_{T}$, (b) $2^{\text {nd }}$ jet $E_{T}$, (c) event $H_{T}$, (d) $1^{\text {st }}$ jet fragmentation function $Z$, (e) $2^{\text {nd }}$ jet fragmentation function $Z$, (f) event $\mathbb{E}_{T} \ldots \ldots \ldots$

B.23 Kinematic distributions in CR2 for ST+JP events: (a) minimum of the difference in $\phi$ between $\vec{\not}_{T}^{t r}$ and each jet, (b) maximum of the difference in phi between two jets directions, (c) maximum of the difference in $R$ space between any two jets, (d) $H_{T}-\mathscr{E}_{T}$, (e) $H_{T}-\mathbb{E}_{T}$, (f) $\not_{T} / \mathbb{E}_{T} \ldots .$.

B.24 Kinematic distributions in CR2 for ST+JP events: (a) output distribution of $\mathrm{ANN}_{\text {trackMET }}$, (b) event $\not P_{T}^{t r}$, (d) output distribution of $\mathrm{ANN}_{\mathrm{SIG}}$. . .

B.25 Kinematic distributions in CR3 for ST+JP events: (a) $1^{\text {st }}$ jet $E_{T}$, (b) $2^{\text {nd }}$ jet $E_{T}$, (c) event $H_{T}$, (d) $1^{\text {st }}$ jet fragmentation function $Z$, (e) $2^{\text {nd }}$ jet fragmentation function $Z$, (f) invariant transverse mass of all jets and $\mathbb{E}_{T}$

B.26 Kinematic distributions in CR3 for ST+JP events: (a) minimum of the difference in $\phi$ between $\overrightarrow{\not P}_{T}^{t r}$ and each jet, (b) minimum of the difference in $\phi$ between $\vec{E}_{T}$ and each jet, (c) maximum of the difference in $R$ space between any two jets, (d) $\not_{T}-\mathscr{E}_{T}$, (e) $H_{T}-\mathscr{E}_{T}$, (f) $\not H_{T} / \mathscr{E}_{T} \ldots \ldots$ 
Figure

B.27 Kinematic distributions in CR3 for ST+JP events: (a) output distribution of $\mathrm{ANN}_{\text {trackMET }}$, (b) number of jets, (d) output distribution of $\mathrm{ANN}_{\mathrm{SIG}}$

C.1 Correlations between some of the important variables in CR1, Exclusive ST events: (a) $r\left(\min \left(\Delta \phi\left(\vec{E}_{T}, \overrightarrow{J_{i}}\right)\right), \not_{T}^{t r}\right)$, (b) $r\left(\max \left(\Delta \phi\left(\vec{J}_{i}, \vec{J}_{k}\right)\right), \not_{T}\right)$, (c) $r\left(\notin_{T}, \not t_{T}^{t r}\right),(\mathrm{d}) r\left(\notin_{T}, M_{j j}\right),(\mathrm{e}) r\left(\Delta \phi\left(\overrightarrow{\phi_{T}}, \overrightarrow{\not_{T}^{t r}}\right), M_{j j}\right)$,

(f) $r\left(\max \left(\Delta R\left(\vec{J}_{i}, \vec{J}_{k}\right)\right), \not_{T}^{t r}\right) \ldots \ldots \ldots \ldots$

C.2 Correlations between some of the important variables in CR2, Exclusive ST events: (a) $r\left(\Delta \phi\left(\overrightarrow{\mathbb{E}_{T}}, \overrightarrow{P_{T}^{t r}}\right), \mathbb{E}_{T}\right),(\mathrm{b}) \min \left(\Delta \phi\left(\overrightarrow{\mathscr{E}_{T}}, \overrightarrow{J_{i}}\right), M_{j j}\right),(\mathrm{c}) r\left(\mathbb{E}_{T}, \not_{T}^{t r}\right)$, (d) $r\left(\left.\right|_{T}, M_{j j}\right)$, (e) $r\left(\Delta \phi\left(\overrightarrow{\mathbb{E}_{T}}, \overrightarrow{P_{T}^{t r}}\right), \not P_{T}^{t r}\right)$, (f) $r\left(\Delta \phi\left(\overrightarrow{\mathbb{E}_{T}}, \overrightarrow{\not_{T}^{t r}}\right), M_{j j}\right) \ldots \ldots$

C.3 Correlations between some of the important variables in CR1, ST+ST events: (a) $r\left(\min \left(\Delta \phi\left(\overrightarrow{\mathscr{E}_{T}}, \overrightarrow{J_{i}}\right)\right), \not_{T}^{t r}\right)$, (b) $r\left(\max \left(\Delta \phi\left(\overrightarrow{J_{i}}, \overrightarrow{J_{k}}\right)\right), \notin_{T}\right)$,

(c) $r\left(\notin_{T}, \not t_{T}^{t r}\right),(\mathrm{d}) r\left(\notin_{T}, M_{j j}\right),(\mathrm{e}) r\left(\Delta \phi\left(\overrightarrow{\phi_{T}}, \overrightarrow{\not_{T}^{t r}}\right), M_{j j}\right)$,

(f) $r\left(\max \left(\Delta R\left(\vec{J}_{i}, \vec{J}_{k}\right)\right), \not_{T}^{t r}\right) \ldots \ldots \ldots \ldots$

C.4 Correlations between some of the important variables in CR2, ST+ST events: (a) $r\left(\Delta \phi\left(\overrightarrow{\mathscr{E}_{T}}, \overrightarrow{\not_{T}^{t r}}\right), \notin_{T}\right)$, (b) $\min \left(\Delta \phi\left(\overrightarrow{\mathbb{E}_{T}}, \overrightarrow{J_{i}}\right), M_{j j}\right)$, (c) $r\left(\mathbb{E}_{T}, \not_{T}^{t r}\right)$, (d) $r\left(\left.\right|_{T}, M_{j j}\right),(\mathrm{e}) r\left(\Delta \phi\left(\overrightarrow{\mathbb{E}_{T}}, \overrightarrow{\not_{T}^{t r}}\right), \not \not_{T}^{t r}\right),(\mathrm{f}) r\left(\Delta \phi\left(\overrightarrow{\mathbb{E}_{T}}, \overrightarrow{\not_{T}^{t r}}\right), M_{j j}\right) \ldots \ldots$

C.5 Correlations between some of the important variables in CR1, ST+JP events: (a) $r\left(\min \left(\Delta \phi\left(\overrightarrow{\mathbb{E}_{T}}, \overrightarrow{J_{i}}\right)\right), \not p_{T}^{t r}\right)$, (b) $r\left(\max \left(\Delta \phi\left(\overrightarrow{J_{i}}, \overrightarrow{J_{k}}\right)\right), \mathscr{E}_{T}\right)$,

(c) $r\left(\notin_{T}, \not t_{T}^{t r}\right),(\mathrm{d}) r\left(\notin_{T}, M_{j j}\right),(\mathrm{e}) r\left(\Delta \phi\left(\overrightarrow{\mathbb{E}_{T}}, \overrightarrow{\not_{T}^{t r}}\right), M_{j j}\right)$,

(f) $r\left(\max \left(\Delta R\left(\vec{J}_{i}, \overrightarrow{J_{k}}\right)\right), \not_{T}^{t r}\right)$

C.6 Correlations between some of the important variables in CR2, ST+JP events: (a) $r\left(\Delta \phi\left(\overrightarrow{\mathscr{E}_{T}}, \overrightarrow{P_{T}^{t r}}\right), \mathbb{E}_{T}\right)$, (b) $\min \left(\Delta \phi\left(\overrightarrow{\mathbb{E}_{T}}, \overrightarrow{J_{i}}\right), M_{j j}\right)$, (c) $r\left(\mathbb{E}_{T}, \not_{T}^{t r}\right)$, (d) $r\left(\not_{T}, M_{j j}\right)$, (e) $r\left(\Delta \phi\left(\overrightarrow{\mathscr{E}_{T}}, \overrightarrow{\not_{T}^{t r}}\right), \not \not_{T}^{t r}\right)$, (f) $r\left(\Delta \phi\left(\overrightarrow{\mathscr{E}_{T}}, \overrightarrow{\not_{T}^{t r}}\right), M_{j j}\right) \ldots \ldots$

D.1 Significance of the sample as a function of the cut on $\mathrm{ANN}_{\mathrm{MJ}}$ output: (a) Exclusive ST, (b) ST+ST, (c) ST+JP. . . . . . . . . . . . . 208

D.2 Correlations between the $\mathrm{ANN}_{\mathrm{MJ}}$ input variables, for (a) background and (b) signal samples.

D.3 Correlations between the $\mathrm{ANN}_{\mathrm{SIG}}$ input variables for 2 jet events, for (a) background and (b) signal samples. . . . . . . . . . . .

D.4 Correlations between the $\mathrm{ANN}_{\mathrm{SIG}}$ input variables for 3 jet events, for (a) background and (b) signal samples. . . . . . . . . . . . . . . . 


\begin{abstract}
Apresyan, Artur. Ph.D., Purdue University, May, 2009. Search for the Standard Model Higgs boson in the Missing Transverse Energy and b-jet signature in protonantiproton collisions at a center of mass energy of $1.96 \mathrm{TeV}$. Major Professor: Daniela Bortoletto.
\end{abstract}

We report on the results of a search for the standard model Higgs boson produced in association with a $W$ or $Z$ boson in $p \bar{p}$ collisions at $\sqrt{s}=1.96 \mathrm{TeV}$ recorded by the CDF II experiment at the Tevatron in a data sample corresponding to an integrated luminosity of $2.1 \mathrm{fb}^{-1}$. We consider events having no identified charged leptons, a large imbalance in transverse momentum, and two or three jets where at least one jet contains a secondary vertex consistent with the decay of a $b$ hadron. The main backgrounds are modeled with innovative techniques using data. The sensitivity of the search is optimized using multivariate discriminant techniques. We find good agreement between data and the standard model predictions. We place 95\% confidence level upper limits on production cross section times branching ratio for several Higgs boson masses ranging from $110 \mathrm{GeV} / c^{2}$ to $150 \mathrm{GeV} / c^{2}$. For a mass of $115 \mathrm{GeV} / c^{2}$ the observed (expected) limit is 6.9 (5.6) times the standard model prediction. 


\title{
Chapter 1. The Standard Model of Particle Physics
}

\author{
By convention sweet, by convention bitter, \\ By convention hot, by convention cold, \\ By convention color: but in reality atoms and void
}

Democritus (circa 400 B.C.)

The goal to understand the world around us has been driving humankind since the beginning of conscious thinking. Various theoretical models of the rules governing the Universe were proposed in the past. This gave rise to a need for a method that could distinguish the correct model from the rest. The "experimental method" was developed in the process, which required an accumulation of experimental facts that could serve as a proof in a scientific inquiry. One of the questions that physicists and philosophers alike pondered on, was on the question of what matter is. In this dissertation we attempt to reassure one of the most ambitious theories describing matter at its most fundamental level, the standard model of particle physics.

Until the beginning of the $19^{\text {th }}$ century the majority of scientific explorations of matter focused on identification of various elements and their chemical interactions. A new era of the understanding of the basic constituents of the matter opened when in 1803 Dalton revived the study of matter, stating that atoms are the fundamental building blocks of Nature, which combine to form chemical compounds. The era of of the elementary particle physics started with the discovery of an electron by J.J.Thomson [1], and the understanding of the microscopic world was revolutionized in 1911 after E.Rutherford's experiments on gold atoms [2]. Another revolution was the discovery of the quark structure of the proton at Stanford Linear Accelerator Center in 1968, which was essentially a scattering experiment of electrons on protons, similar to Rutherford's foil experiments. The big difference between these two experiments, 
however, was that they probed different scales: the typical size of an atom is $\sim 1 \AA=$ $10^{-10} \mathrm{~m}$, while for proton it is about $1 \mathrm{fm}=10^{-15} \mathrm{~m}$. As follows from the uncertainty

principle, $\Delta E \Delta x \gtrsim \hbar c \sim 0.2 \mathrm{GeV} \cdot \mathrm{fm}$, in order to probe smaller distances one needs to increase the energies. To see a structure inside of a proton $(\Delta x \ll 1 f m)$, therefore, requires energies of $E \gg 1 \mathrm{GeV}$. It is this need for higher energies that separated the two discoveries by half a century, and the need to probe even smaller distances has determined design of various experimental apparatuses which were built since then. Another reason for going to higher energies is to enable studies of some of the elementary particles, which are too heavy to exist as free particles, but nevertheless play an important role in nature, such as $W^{ \pm} / Z$ bosons or the top quark.

A crucial role in the advancement of particle physics has been played by collider experiments performed at synchrotron accelerators. In this machines beams of particles, such as $e^{+} e^{-}$or $p \bar{p}$ are accelerated to high speed and made to collide. High energies achieved in such collisions allow the production and study of elementary particles or searches for new particles predicted by theory. The work presented in this dissertation is based on experimental data collected in proton-antiproton collisions, recorded at CDF experiment, which operates at the Tevatron accelerator at center-of-mass energy $\sqrt{s}=1.96 \mathrm{TeV}$.

\subsection{Introduction}

At our current level of understanding the basic constituents of matter are the quarks and leptons, which are spin 1/2 particles, called fermions. There are four basic interactions among these particles - gravitational, weak, electromagnetic, and strong. The interactions between fermions are mediated by spin 1 particles, which are called bosons. All the quarks and leptons, as well as the force carriers have been experimentally observed. These particles have masses varying over a very wide range, from $511 \times 10^{-6} \mathrm{GeV} / c^{2}$ for an electron to the mass of the heaviest observed elementary particle: the top quark with a mass of around $172 \mathrm{GeV} / c^{2}$. One of the biggest 
questions of the modern physics is to understand the mechanism which gives masses to the elementary particles. Lagrangian density with explicit mass terms for any of the particles would violate the gauge symmetry. However, if the gauge symmetry is imposed, then the $W^{ \pm} / Z$ bosons that mediate the electro-weak interactions are required to be massless and the fermions' masses are required to be zero as well.

The way to give a mass to $W^{ \pm} / Z$ bosons, while preserving a local gauge symmetry, leads to the concept of the spontaneous symmetry breaking. This concept does not rely on mass terms in the Lagrangian, but rather on the assumption that there exists a scalar field with a specific form of interaction responsible for the mass of all the particles. Particles then acquire mass as a result of the interaction with this scalar field, which extends over all space-time continuum [3].

The spontaneous symmetry breaking, called the "Higgs mechanism" if applied to the local symmetry, is then considered as an "origin" of the mass of fermions and gauge bosons. The existence of the Higgs scalar is expected to be the direct physical manifestation of this mechanism. Search for the Higgs boson is thus currently one of the most active areas of research in experimental particle physics.

A brief overview of the Standard Model of particle physics is given in this chapter. The Higgs mechanism is introduced in Sec. 1.4. The current state of the theoretical and experimental bounds and various searches of the Higgs boson is presented in Sec. 1.6.

\subsection{Particles and Fields in the Standard Model}

The Standard Model (SM) of particle physics is a consistent and calculable (within the limitations of current technical abilities) theory of fundamental interactions that successfully explains most of the known phenomena in elementary particle physics $[4,5]$. The SM describes weak, electromagnetic and strong interactions. Although it is desired to have a unified treatment of all known forces including gravity, at 
the energies currently accessible in particle physics experiments the role of gravity is negligible.

The SM describes the microscopic world in terms of interacting particles and fields in the framework of relativistic quantum field theory [6]. In this framework one postulates the set of symmetries of the system and constructs the most general Lagrangian from the particle and field content of the theory that obeys these symmetries. All relativistic quantum field theories must obey the global Poincaré symmetry.

The choice of the symmetries essentially defines the theory, so this choice has to be made carefully. Early in the development of quantum field theories it was noticed that divergent integrals appeared in many perturbative calculations, such as in the process $e^{+} e^{-} \rightarrow \mu^{+} \mu^{-}$shown in Fig. 1.1 .
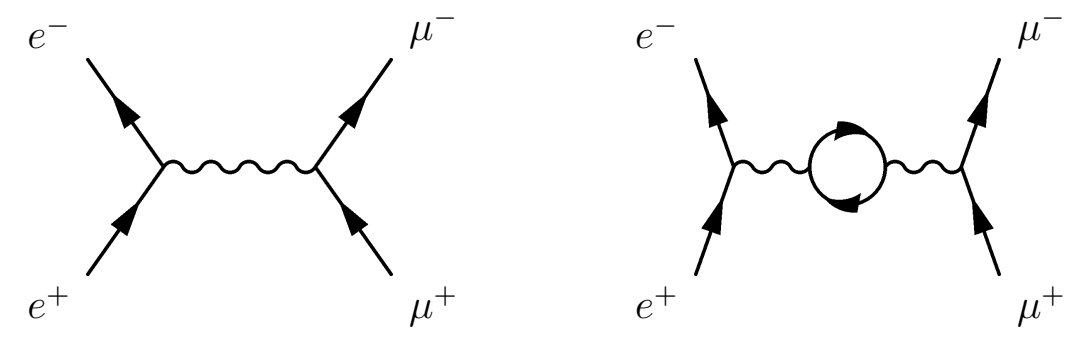

Figure 1.1.: $e^{+} e^{-} \rightarrow \mu^{+} \mu^{-}$scattering at the leading order (left), and with one loop (right)

The amplitude of this process at the leading order is of the order of $\propto \alpha=\frac{e^{2}}{4 \pi}$, the fine structure constant. However, when including the next order of perturbative expansion, a diagram like the one shown on the right in Fig. 1.1 also contributes. A pair of particle-antiparticle is created inside such a loop. The momentum of the particles in the loop is not determined by the momentum of the incoming and outgoing particles. Therefore, to find the amplitude of such a process one needs to integrate over all possible values of energy and momentum of the particles inside the loop. This procedure leads to a problem though, since such an integral diverges logarithmically!

The solution of this and other divergences was found to be a procedure called renormalization [7]. The procedure involves separating out the parts which are di- 
vergent, and getting rid of those divergences which turn out to be non-physical. The procedure of renormalization does not work for all types of fields though, so it serves as a guiding principle when constructing field theories. It was shown in the beginning of 1970's that the type of theories called gauge theories can be renormalized $[8,9]$. On the other hand, the classical theory of gravity, general relativity, becomes nonrenormalizable if one tries to treat it in the framework of quantum field theories. The standard model of particle physics is a renormalizable theory based on the gauge group:

$$
S U(3) \otimes S U(2) \otimes U(1)
$$

The three groups correspond to three fundamental interactions: $S U(3)$ describes the strong interactions in Quantum Chromodynamics (QCD), $S U(2)$ corresponds to the weak interactions and the group $U(1)$ gives rise to the electromagnetic interactions of the Quantum Electrodynamics (QED). All of the so far observed evidence suggests that all the phenomena in microscopic world are governed by a combination of these forces.

The particle content of the SM and their properties [10] are summarized in the Tab. 1.1. In the SM the matter fields are all fermions and are organized in 3 generations with identical quantum numbers but different masses.

$$
\left[\begin{array}{llll}
u & u & u & \nu_{e} \\
d & d & d & e
\end{array}\right] \quad\left[\begin{array}{llll}
c & c & c & \nu_{\mu} \\
s & s & s & \mu
\end{array}\right] \quad\left[\begin{array}{llll}
t & t & t & \nu_{\tau} \\
b & b & b & \tau
\end{array}\right]
$$

The pattern is shown in Eq.1.1. Each family contains three pairs of quarks with different color and a colorless doublet with a neutrino and a charged lepton. At our present level of understanding it is not clear why the Nature chose these three repetitions of fermion groups. The interactions between the particles in SM are mediated by spin 1 particles which are called gauge bosons: the eight gluons of the strong interactions, the photon of the electromagnetic interactions and the $W^{+}, W^{-}$ and $Z^{0}$ of the weak interactions. An example Feynman diagram for each type of 
interaction in SM is presented in Fig.1.2 along with the corresponding scattering amplitudes.
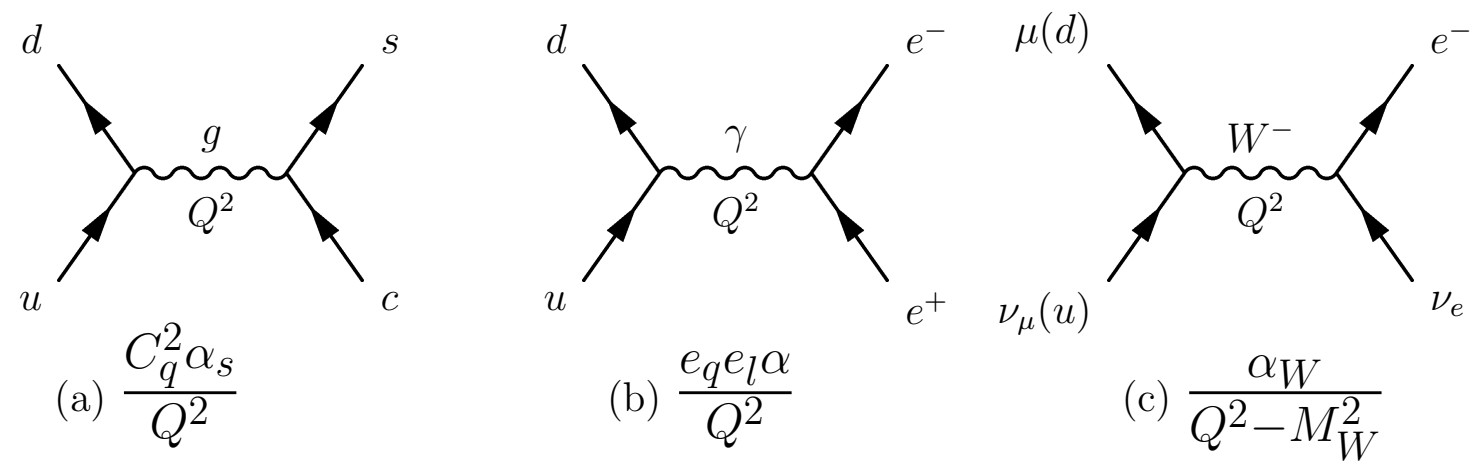
(b) $\frac{e_{q} e_{l} \alpha}{Q^{2}}$
(c) $\frac{\alpha_{W}}{Q^{2}-M_{W}^{2}}$

Figure 1.2.: Feynman diagrams for (a) strong, (b) electromagnetic and (c) weak interactions. $Q^{2}$ is the square of 4-momentum transferred between the particles.

The gluon coupling is proportional to the color charge $C_{q}$, similar to the situation in electrodynamics, where the coupling is proportional to the electric charge $e_{q}$. In the case of strong interactions the coefficient of proportionality is labeled as $\alpha_{s}$ similar to fine structure constant $\alpha$ in electrodynamics. However, unlike the situation in QED, the force carriers in QCD are colored, hence self-coupled. As a result of this selfinteraction, the strong force increases linearly with distance, making quarks tightly bound inside hadrons, since it would require an infinite amount of energy to split them apart. The impossibility of separating color charges, such as individual quarks and gluons, is called color confinement. So far, no free quarks or gluons have been observed, and they occur in bound states which are color-neutral: states with three quarks all of different color (baryons) and a pair of color-anticolor quarks (mesons).

When quarks or gluons are created in an interaction, they are never observed in a free state, since they quickly transform to colorless states. For example if a pair of quarks is created in a collision, the final state quark and antiquark have large energies, and travel in opposite directions very fast. Color confinement forces create new quarkantiquark pairs from vacuum which combine to create color-neutral mesons. Or one of the quarks radiates a gluon, which in turn radiates a quark pair and so on. The 
Table 1.1: Properties of elementary particles in the Standard Model

\begin{tabular}{|c|c|c|c|c|}
\hline & Particle Name & $\operatorname{Mass}\left(\mathrm{GeV} / c^{2}\right)$ & Charge & Interaction \\
\hline \multirow{6}{*}{ Leptons } & electron $(e)$ & $511 \times 10^{-6}$ & \pm 1 & EM, Weak \\
\hline & $e$ neutrino $\left(\nu_{e}\right)$ & $<2.3 \times 10^{-6}$ & 0 & Weak \\
\hline & muon $(\mu)$ & $106 \times 10^{-3}$ & \pm 1 & EM, Weak \\
\hline & $\mu$ neutrino $\left(\nu_{\mu}\right)$ & $<0.17 \times 10^{-3}$ & 0 & Weak \\
\hline & $\operatorname{tau}(\tau)$ & 1.78 & \pm 1 & EM, Weak \\
\hline & $\tau$ neutrino $\left(\nu_{\tau}\right)$ & $<15.5 \times 10^{-3}$ & 0 & Weak \\
\hline \multirow{6}{*}{ Quarks } & up $(u)$ & $(1.5-3.3) \times 10^{-3}$ & $\pm 2 / 3$ & Strong, EM, Weak \\
\hline & down $(d)$ & $(3.5-6.0) \times 10^{-3}$ & $\mp 1 / 3$ & Strong, EM, Weak \\
\hline & $\operatorname{charm}(c)$ & $1.27_{-0.11}^{+0.07}$ & $\pm 2 / 3$ & Strong, EM, Weak \\
\hline & strange $(s)$ & $(70-130) \times 10^{-3}$ & $\mp 1 / 3$ & Strong, EM, Weak \\
\hline & top $(t)$ & $171.2 \pm 2.1$ & $\pm 2 / 3$ & Strong, EM, Weak \\
\hline & bottom $(b)$ & $4.20_{-0.07}^{+0.17}$ & $\mp 1 / 3$ & Strong, EM, Weak \\
\hline \multirow{4}{*}{ Gauge Bosons } & gluon $(g)$ & 0 & 0 & Strong \\
\hline & photon $(\gamma)$ & 0 & 0 & EM \\
\hline & $\mathrm{W}$ boson $\left(W^{ \pm}\right)$ & $80.40 \pm 0.03$ & \pm 1 & Weak \\
\hline & $\mathrm{Z}$ boson $\left(Z^{0}\right)$ & $91.188 \pm 0.002$ & 0 & Weak \\
\hline
\end{tabular}

new partons are nearly collinear with the original parton, and what is observed is a spray of color-less particles which move in the same direction. This process is referred to as fragmentation and the spray of collimated particles is called a jet.

The coupling constant $\alpha_{s}$ in QCD is a function of transferred momentum $Q^{2}$ which decreases with increasing $Q^{2}$ and vanishes asymptotically. This leads to the property of QCD called asymptotic freedom and allows to calculate the interaction of elementary particles using perturbative methods. Since the momentum in collisions in modern particle accelerators is very high, it is possible to calculate the interactions using perturbative expansions. However, in the process of jets formation the energies 
of the particles are successively smaller, and perturbative QCD is no longer applicable. Phenomenological models are usually applied in order to perform the calculations in such energy regimes.

The weak interaction is mediated by massive particles: the $W^{ \pm}$and $Z^{0}$ bosons. Since the force carrier in the case of weak interactions is a massive particle, it follows from the uncertainty principle that this interactions are restricted to a short range

$$
\Delta E=M_{W} \cdot c^{2} \Rightarrow \Delta x \sim \frac{\hbar}{M_{W} \cdot c}<1 \mathrm{fm}
$$

\subsection{Electroweak Unification}

The earliest successful attempt in constructing a quantum field theory was the development of the Quantum Electrodynamics (QED), which describes electromagnetic interactions. As was discussed above, the interaction need to be gauge invariant in order for the theory to be renormalizable, and QED is a gauge invariant theory described by the $U(1)$ symmetry group.

The invariance of classic electrodynamics under the gauge transformation:

$$
A_{\mu} \rightarrow A_{\mu}-\frac{1}{e} \partial_{\mu} \alpha(x)
$$

is recast into QED in terms of group theory. The Lagrangian of a free Dirac particle,

$$
\mathscr{L}=\bar{\Psi}\left(i \gamma^{\mu} \partial_{\mu}-m\right) \Psi
$$

is invariant under global $U(1)$ transformations $\Psi \rightarrow e^{-i \alpha} \Psi$. However, it is not invariant under local $U(1)$ transformations $\Psi \rightarrow e^{-i \alpha(x)} \Psi$, where now $\alpha(x)$ is a function of space-time. By replacing the derivative in Eq. 1.2 with the covariant derivative

$$
D_{\mu} \equiv \partial_{\mu}+i e A_{\mu}
$$

where a real field $A_{\mu}$ transforms as in Eq. 1.2, the Lagrangian becomes invariant under $U(1)$ transformations. For completeness a kinetic term is introduced:

$$
\mathscr{L}^{K . E .}=-\frac{1}{4} F_{\mu \nu} F^{\mu \nu}
$$


where the field tensor $F_{\mu \nu}$ is given by:

$$
F_{\mu \nu}=\partial_{\mu} A_{\nu}-\partial_{\nu} A_{\mu}
$$

The final QED Lagrangian is given by:

$$
\begin{aligned}
\mathscr{L}_{Q E D} & =\bar{\Psi}\left(i \gamma^{\mu} D_{\mu}-m\right) \Psi-\frac{1}{4} F_{\mu \nu} F^{\mu \nu} \\
& =\bar{\Psi}\left(i \gamma^{\mu} \partial_{\mu}-m\right) \Psi-e \bar{\Psi} \gamma^{\mu} \Psi A_{\mu}-\frac{1}{4} F_{\mu \nu} F^{\mu \nu}
\end{aligned}
$$

As it can be seen from the second line in Eq. 8.3 there is a term $e \bar{\Psi} \gamma^{\mu} \Psi A_{\mu}$ that describes the interaction between the charged particles, represented by the wave function $\Psi$ and the gauge field $A_{\mu}$. The requirement of the gauge invariance has transformed the free system into a system of interacting particles and fields! In a sense, the existence and properties of the photon follow from the requirement of local gauge invariance under $U(1)$ transformations. Note that the Lagrangian does not contain a mass term for the field $A_{\mu}$, such as $m^{2} A_{\mu} A^{\mu}$ since such a term would violate local gauge invariance. Therefore, the photon is massless in QED.

The electroweak theory, which unifies the weak and electromagnetic interactions is constructed similarly to QED. The theory of weak interactions was initially developed to explain the properties of the $\beta$-decay by E.Fermi in 1934 [11]. In an analogy with electromagnetic interactions in QED, such as electron proton scattering, whose amplitude is given by a product of electron and proton currents:

$$
\mathcal{M}=\left(e \bar{u}_{p} \gamma^{\mu} u_{p}\right)\left(-\frac{1}{q^{2}}\right)\left(-e \bar{u}_{e} \gamma_{\mu} u_{e}\right)
$$

Fermi proposed a theory of $\beta$-decays with similar amplitudes:

$$
\mathcal{M}=G_{F}\left(\bar{u}_{n} \gamma^{\mu} u_{p}\right)\left(\bar{u}_{\nu_{e}} \gamma_{\mu} u_{e}\right)
$$

for the charged currents of weak decays. Fermi's model provided a satisfactory description of the phenomenology of $\beta$-decays, but it was soon evident that the it had some problems, e.g. indefinite growth of the cross-sections as the energy increases.

After the discovery of the parity violation in weak interactions it was realised that the vector-vector interaction term in Fermi's theory had to be modified, with 
the replacement of $\gamma^{\mu}$ in Eq. 1.9, by $\gamma^{\mu}\left(1-\gamma^{5}\right)[12,13]$. In fact, parity was violated maximally, and weak interaction affected only left-handed particles. All the experimental evidence until now suggests that neutrinos indeed interact with leptons only through weak interaction, and therefore are left-handed. The weak interaction term is customarily referred to as the " $V-A$ " structure of weak interactions.

The first attempt to incorporate the $V-A$ structure into a gauge theory was made by Bludman in 1958 [14]. His model, based on the $S U(2)$ weak isospin group, required three vector bosons (number of generators of the group) and predicted a neutral massive boson. The detection of neutrino scattering in CERN experiments in 1973 [15] opened a new chapter in particle physics, with the observation of weak neutral currents.

The next step of unifying the electromagnetic and weak interactions was made by Glashow in 1961 [16], when he utilized the gauge group $S U(2) \otimes U(1) . U(1)$ was associated to the weak hypercharge $Y_{W}$ and related to the weak isospin $(I)$ and electric charge $(Q)$ through

$$
Q=I_{3}+\frac{Y_{W}}{2}
$$

This theory required four gauge bosons: a triplet $\left(W_{\mu}^{1}, W_{\mu}^{2}, W_{\mu}^{3}\right)$ corresponding to the $S U(2)$ group, and a neutral field $B_{\mu}$ corresponding to $U(1)$. Similar to the QED, a covariant derivative was introduced:

$$
D_{\mu} \equiv \partial_{\mu}+i g \frac{\tau_{a}}{2} W_{\mu}^{a}+i \frac{g^{\prime}}{2} Y_{W} B_{\mu}
$$

where $g$ and $g^{\prime}$ are the coupling constants and $\tau^{a}$ are Pauli spin matrices. As a result, the charged weak currents appeared as linear combinations of $W_{\mu}^{1}$ and $W_{\mu}^{2}$, while the photon and the neutral vector boson $Z$ as mixture of $W_{\mu}^{3}$ and $B_{\mu}$.

$$
\begin{aligned}
W_{\mu}^{ \pm} & \equiv \sqrt{\frac{1}{2}}\left(W_{\mu}^{1} \mp W_{\mu}^{2}\right) \\
A_{\mu} & \equiv B_{\mu} \cos \theta_{W}+W_{\mu}^{3} \sin \theta_{W} \\
Z_{\mu} & \equiv-B_{\mu} \sin \theta_{W}+W_{\mu}^{3} \cos \theta_{W} \\
\frac{g^{\prime}}{g} & =\tan \theta_{W}
\end{aligned}
$$


The coupling of electromagnetic current to the photon field $A^{\mu}$ was given by electric charge:

$$
Q=g \cdot \sin \theta_{W}=g^{\prime} \cdot \cos \theta_{W}
$$

However, the masses of the vector bosons in this theory were introduced "by hand", breaking the gauge invariance of the theory. The problem was overcome by employing the idea of spontaneous symmetry breaking, the "Higgs mechanism".

\subsection{The Higgs Mechanism}

The Higgs mechanism was proposed by P. Higgs in 1964 [17] and implemented to give masses to $W$ and $Z$ bosons by Weinberg and Salam [18,19] in the $S U(2) \otimes$ $U(1)$ theory. The mechanism is best illustrated in the case of $U(1)$ gauge invariant Lagrangian for a complex scalar field, which will be shown in this section.

Let us consider the Higgs mechanism in for a system containing a gauge boson $A^{\mu}$. Here one introduces one complex scalar boson field $\Phi$ :

$$
\Phi=\frac{1}{\sqrt{2}}\left(\phi_{1}+i \phi_{2}\right)
$$

The interaction with the gauge boson is described by the Lagrangian density with a local gauge group $U(1)$ in the following form:

$$
\mathscr{L}=\left(D_{\mu} \Phi\right)\left(D^{\mu} \Phi\right)^{*}+\mu^{2} \Phi^{*} \Phi-\lambda\left(\Phi^{*} \Phi\right)^{2}-\frac{1}{4} F^{\mu \nu} F_{\mu \nu}
$$

where $F^{\mu \nu}$ is defined in Eq. 8.2. The covariant derivative

$$
D^{\mu}=\partial^{\mu}+i g A^{\mu}
$$

contains the term related to the interaction between the scalar and the gauge field with a coupling $g$ (similar to the electric charge in Eq. 7.3). The considered Lagrangian density is manifestly symmetric under the local $U(1)$ symmetry transformation and does not contain any explicit mass terms.

The parameters in the potential part:

$$
V=-\mu^{2} \Phi^{*} \Phi+\lambda\left(\Phi^{*} \Phi\right)^{2},
$$


are:

$$
\mu^{2}>0 \quad \text { and } \lambda>0
$$

leading to a potential bounded from below. Note that a negative $\mu^{2}$ would correspond to the mass term for $\Phi$. The potential 8.4 now has a circle of minima with a radius $v$ :

$$
v=\phi_{1}^{2}+\phi_{2}^{2}=\sqrt{\frac{\mu^{2}}{\lambda}},
$$

where $v$ is called vacuum expectation value. By choosing one of these minima as a a true minimum of the energy, the symmetry of the physical system is spontaneously broken, since the Lagrangian $\mathscr{L}$ is invariant under this transformation but the vacuum is not.

The original $\Phi(x)$ field can be expressed by new real fields, $\xi$ and $h$, with zero vacuum expectation values, as in:

$$
\Phi(x)=\frac{e^{i \xi / v}}{\sqrt{2}}(v+h(x)),
$$

By choosing a gauge with $\xi=0$ we get

$$
\begin{array}{r}
\mathscr{L}=\frac{1}{2}\left(\partial_{\mu}-i g A_{\mu}\right)(v+h)\left(\partial^{\mu}+i g A^{\mu}\right)(v+h) \\
+\frac{\mu^{2}}{2}(v+h)^{2}-\frac{\lambda}{4}(v+h)^{4}-\frac{1}{4} F^{\mu \nu} F_{\mu \nu}= \\
\frac{1}{2}\left(\partial_{\mu} h\right)\left(\partial^{\mu} h\right)-\mu^{2} h^{2}+\frac{(g v)^{2}}{2} A^{\mu} A_{\mu}+g^{2} v h A_{\mu} A^{\mu}+. .
\end{array}
$$

Interpreting the individual terms in the Lagrangian density $\mathscr{L}$ one can find that the theory contains:

- a mass term for the gauge boson $M=g v$,

- a neutral scalar boson $h$ (a real field) with a mass $\sqrt{2} \mu$,

- the interaction terms $g M h A^{\mu} A_{\mu}$ with the coupling proportional to the mass of the gauge boson,

- the self interaction terms $h h h$, hhhh etc. 
By measuring the gauge boson mass one can determine the parameter $v$, provided there is independent constraint on the coupling $g$ :

$$
M=g v .
$$

The second term in 1.23 predicts a scalar particle, with mass $\sqrt{2} \mu$, which is called Higgs boson. However to obtain the mass of the Higgs boson we should know the self interaction, i.e. parameter $\lambda$, since

$$
m_{H}=\sqrt{2 \lambda} v .
$$

To generalize the Higgs mechanism for $S U(2) \otimes U(1)$ group we introduce a complex scalar $S U(2)$ doublet

$$
\Phi=\left(\begin{array}{c}
\phi^{+} \\
\phi^{0}
\end{array}\right),
$$

The electroweak sector in SM is described by a covariant derivative:

$$
D^{\mu}=\partial^{\mu}+i g \frac{\vec{\tau}}{2} \vec{W}^{\mu}+i g^{\prime} \frac{Y_{W}}{2} B^{\mu}
$$

where the ratio of couplings $g$ and $g^{\prime}$ is described by the Weinberg angle $\theta_{W}, \tan \theta_{W}=$ $g^{\prime} / g$. The original vector gauge fields:

$$
W_{1}^{\mu}, W_{2}^{\mu} \text { and } W_{3}^{\mu}, B^{\mu}
$$

after mixing between the neutral fields, lead to the following physical charged and neutral fields

$$
W_{\mu}^{+}, W_{\mu}^{-} \text {and } Z_{\mu}, A_{\mu}
$$

with the corresponding particles known as $W^{ \pm}, Z$ bosons and the photon, $\gamma$. They mediate the charged (CC), neutral current (NC) processes and electromagnetic processes, respectively. Their masses are then predicted by the resulting Lagrangian:

$$
\begin{aligned}
M_{W} & =\frac{1}{2} g v \\
M_{Z} & =\frac{1}{2} v \sqrt{g^{2}+g^{\prime 2}} \\
\frac{M_{W}}{M_{Z}} & =\cos \theta_{W} \\
M_{A} & =0
\end{aligned}
$$


As noted above, in 1973 Gargamelle collaboration at CERN performed the first measurement of neutrino-induced weak neutral currents. From the measurement of the ratio of Neutral Currents (NC) to Charged Currents (CC) [15] it was found that at $90 \%$ C.L.

$$
0.1<\sin \theta_{W}^{2}<0.6
$$

From low-energy phenomenology one can obtain a relation [4]

$$
\frac{g}{2 \sqrt{2}}=\sqrt{\frac{M_{W}^{2} G_{F}}{\sqrt{2}}}
$$

which together with 1.15 provided the first hints of the mass of $\mathrm{W}$ and $\mathrm{Z}$ bosons:

$$
M_{W}^{2}=\frac{Q^{2}}{4 \sin \theta_{W}^{2}} v^{2} \approx\left(\frac{37.2}{\sin \theta_{W}} \mathrm{GeV}\right)^{2} \sim(48-118 \mathrm{GeV})^{2}
$$

and a similar relation may be obtained for $M_{Z}$ :

$$
M_{Z}^{2} \approx\left(\frac{37.2}{\sin \theta_{W} \cos \theta_{W}} G e V\right)^{2} \sim(76-124 G e V)^{2}
$$

In 1983, at the CERN SPS $p \bar{p}$ collider, both $Z[20,21]$ and $W[22,23]$ bosons were discovered. Their masses were determined with a precision of a few $\mathrm{GeV}$ as shown in Tab. 1.2, in a good agreement with SM predictions. Current most precise measurements of $\sin ^{2} \theta_{W}=0.23119 \pm 0.00014$ [10].

Table 1.2: Masses of the $W$ and $Z$ bosons as measured by UA1 and UA2 experiments, and their most precise current values [10].

\begin{tabular}{|c|c|c|c|}
\hline & UA1 & UA2 & Current Value \\
\hline$M_{W}$ & $83.5 \pm 1.1 \pm 2.7$ & $80.2 \pm 0.6 \pm 0.5$ & $80.40 \pm 0.03$ \\
$M_{Z}$ & $93.0 \pm 1.4 \pm 3.0$ & $91.5 \pm 1.2 \pm 1.7$ & $91.188 \pm 0.002$ \\
\hline
\end{tabular}

In addition to giving masses to the gauge bosons, the interaction with the scalar field yields masses to fermions. Namely, for $f_{L}$ being a $S U(2)$ doublet and $f_{R}$ a $S U(2)$ singlet we get a mass term for the fermion $f$

$$
g_{f}\left[\left(\bar{f}_{L} \Phi\right) f_{R}+h c\right] \rightarrow \frac{g_{f} v}{\sqrt{2}}(\bar{f} f) .
$$


Here $g_{f}$ is the so called Yukawa coupling for the fermion $f$. The parameter in front of the bracket in (1.36) can be interpreted as the mass of a fermion $f$, and therefore

$$
m_{f}=\frac{g_{f} v}{\sqrt{2}} .
$$

Therefore, the scalar field generates mass terms for fermions. However the fermions' masses are not fixed by the parameters of the Higgs potential, nor the fermion mass pattern can be driven from the assumed mechanism. Additionally, in spite of predicting the existence of the Higgs boson, the theory does not predict its mass, since the parameter $\lambda$ is a priori unknown.

\subsection{Quantum Chromodynamics}

The theory describing the interactions of quarks and gluons is called Quantum Chromodynamics. After the discovery of the neutron, around 1932, it was realized that the forces keeping nucleons in nuclei together should be different from electromagnetic forces. The first theoretical model of strong interactions was constructed by Yukawa around 1935 [24]. Yukawa assumed that the interactions of nucleons is mediated by a new force, whose quanta, the mesons represent new type of particles. Since the strong interaction is a short-range force, the theory contained massive force carriers, "mesons", with a mass $\sim 200 \mathrm{MeV}$. Another important characteristic of the force was obtained from the realization that since the interaction is strong, the meson-nucleon coupling should be large. The Yukawa theory was confirmed with the discovery of $\pi$-mesons in cosmic rays, with a mass close to that predicted by his model. It was soon however discovered that the model cannot be the ultimate theory of the strong interaction. Many other mesons and baryons were discovered after the pion discovery, and physicist were faced with a problem of classifying the fast growing "zoo of elementary particles and resonances".

It was found that all known hadrons can be grouped into groups of octets and decuplets that can be represented as multiplets of $S U(3)$. The theory was confirmed with the observations of $\Omega^{-}$-hyperon, which was predicted by the theory. The theory 
introduced quarks, which were the particles that belong to the fundamental representation of $S U(3)$. This was the foundation of the quark model of hadrons. In this theory, mesons were formed as a bound state of a quark and anti-quark, while the baryons consist of three quarks. Assuming that the quarks are fermions they were assigned fractional electric charge: $Q_{u}=2 / 3$ and $Q_{d}=-1 / 3$.

The quark model was very successful in describing the known properties of particles and in making predictions. However, the dynamics of the strong force was not clear. It was not understood why the quarks are always in bound states, and many attempts to measure fractional charges were unsuccessful. Another difficulty arose from the observations of $\Delta^{++}$, which according to the quark model should contain $3 u$-quarks in the same quantum state. Since two fermions cannot occupy the same quantum state, according to the Pauli's principle, a new quantum number called color was introduced. It was postulated that only color-less states are allowed, hence quarks are "confined" within hadrons. The color states are conventionally referred to as Red $(R)$, Green $(G)$ and Blue $(B)$.

The relativistic quantum field theory of strong interactions was constructed using the $S U(3)$ group, in a similar fashion to that of weak interactions. The force carriers in QCD are massless gluons. Due to non-Abelian nature of the transformation group, the gluons are self-interacting, which means that gluons can also carry color charge. A breakthrough occurred when the charge renormalization was calculated by Gross, Wilczek and Politzer and the phenomenon of asymptotic freedom was discovered [25, 26]. This allowed to make QCD a self-consistent theory. It also allowed to explain why the strong interactions are strong: if the coupling falls off at small distances it must grow at large distances becoming around 1 at scales $\sim 1 \mathrm{fm}$.

The quark mass eigenstates are not the same as the weak eigenstates that are given by 1.36. The transformation from one eigenstate to another is performed using the Cabibbo-Kobayashi-Maskawa (CKM) matrix. The matrix is not diagonal, and 
therefore it allows mixing of quark flavors. The current most precise values of the CKM matrix are presented in Eq. 1.38 [10]

$$
\left(\begin{array}{c}
d^{\prime} \\
s^{\prime} \\
b^{\prime}
\end{array}\right)=\left(\begin{array}{ccc}
\left|V_{u d}\right| \approx 0.974 & \left|V_{u s}\right| \approx 0.23 & \left|V_{u b}\right| \approx 0.004 \\
\left|V_{c d}\right| \approx 0.230 & \left|V_{c s}\right| \approx 1.04 & \left|V_{c b}\right| \approx 0.04 \\
\left|V_{t d}\right| \approx 0.008 & \left|V_{t s}\right| \approx 0.04 & \left|V_{t b}\right|>0.74
\end{array}\right) \times\left(\begin{array}{c}
d \\
s \\
b
\end{array}\right)
$$

The 9 elements of the CKM matrix can be written in terms of 4 parameters, which need to be determined from experiments: $V_{C K M}=R_{1}\left(\theta_{23}\right) R_{2}\left(\theta_{13}, \delta_{13}\right) R_{3}\left(\theta_{12}\right)$. The $R_{i}\left(\theta_{j k}\right)$ are rotational matrices around the axis $i$, the angle $\theta_{j k}$ describes the mixing of the generations $j$ and $k$ and $\delta_{13}$ is a phase. For three generations the CKM matrix does not have to be real, i.e. the phase $\delta_{13}$ does not have to be zero. This fact explains the $\mathrm{CP}$ violation in weak interactions.

Overall, the Standard Model of particle physics contains 18 free parameters, which need to be determined from experiments: 3 masses of leptons, 6 masses of quarks, 4 parameters of the CKM matrix, gauge couplings of $U(1), S U(2)$ and $S U(3)$, the Higgs quadratic coupling $\mu$ and the Higgs self-coupling $\lambda$.

\subsection{Constraints on the Higgs Boson}

While the Higgs mechanism is successful in generating masses of particles in the SM, the Higgs boson has not yet been discovered. Therefore, the discovery of the Higgs boson is one of the main challenges of experimental particle physics. If the Higgs boson is discovered, it will prove that the standard model of particle physics is correct. Further studies will be required to measure the properties of the Higgs boson, and to see if they match the expectations from the SM. Deviations from the SM will indicate that our knowledge of Nature is not complete and it will help the discovery of new phenomena. If it is found that the Higgs boson does not exist, it will revolutionize our view of the Universe and require that we rethink our approaches. 
The Higgs boson couples to particles through spontaneous symmetry breaking of the $S U(2) \otimes U(1)$. Examples of couplings of the Higgs boson to fermions and boson are listed in Tab. 1.3

Table 1.3: The Higgs boson couplings to fermions and bosons

\begin{tabular}{c|c}
\hline Coupling & Intensity \\
\hline$H f \bar{f}$ & $m_{f} / v$ \\
$H W^{+} W^{-}$ & $m_{W}^{2} / v$ \\
$H Z Z$ & $m_{Z}^{2} / v$ \\
\hline
\end{tabular}

As shown in the Tab.1.3, the Higgs boson couples proportionally to the mass. Therefore, it is most easily produced in association with heavy particles, and its decays branching fractions are the largest for the heavy particles, if kinematically allowed. The Higgs boson can also couple to $\gamma \gamma$ through a loop of charged particles, e.g. $W$ bosons. In a similar way, the Higgs boson can couple to gluons via a quark loop. The couplings of the Higgs boson largely define the experimental techniques that can be used in the searches for the Higgs boson.

Since the Higgs boson has not been observed experimentally, the searches rely on various indirect constraints or theoretical bounds on the Higgs boson mass to guide the direction of the searches. A brief summary of the current state of various searches and limits is presented in the following chapters.

\subsubsection{Theoretical Bounds on the Higgs boson}

It is possible to construct lower bounds on the Higgs boson mass from the requirement of the stability of the Higgs potential. By the requirement that the Standard Model should be valid up to the Planck scale, $\Lambda \sim 10^{19} \mathrm{GeV}$ the following relation can be obtained [27]:

$$
m_{H}>133+1.92\left(m_{t}-175\right)-4.28\left(\frac{\alpha_{s}-0.12}{0.006}\right)
$$


The lower curve on Fig. 1.3 shows the dependence of the Higgs boson mass on the Planck scale $\Lambda$. From this plot, if the Higgs boson is discovered at $m_{H} \simeq 100 \mathrm{GeV}$ the SM would break down around $10^{5} \mathrm{GeV}$.

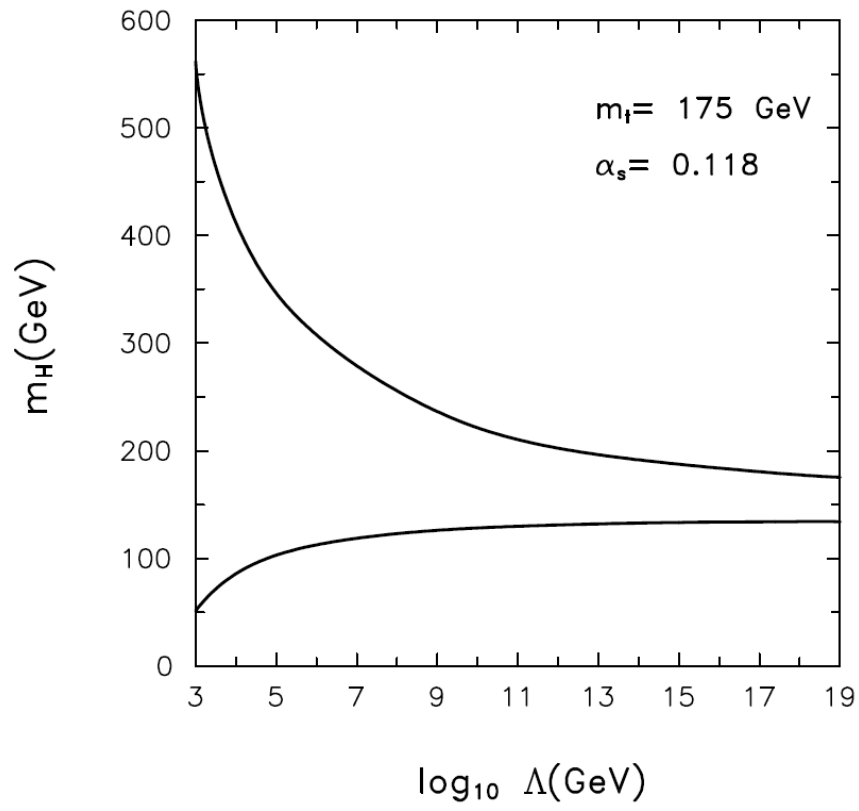

Figure 1.3.: Perturbative and stability bounds on Higgs boson mass as a function of the Planck Scale $\Lambda$ [28]

The upper bounds on the Higgs boson mass can be obtained by requiring that the unitarity is not violated in $W^{+} W^{-}$scattering, which yields an upper limit of $m_{H} \lesssim 1 \mathrm{TeV}$. Another convenient method to obtain the upper bounds for the Higgs boson mass is from the requirement of non-triviality of the Higgs potential, i.e. that the theory does not become non-interacting [29]. The following relation for the quartic coupling $\lambda$ in Eq. 1.16 can be obtained from the renormalization group equation:

$$
\frac{d \lambda}{d t}=\frac{1}{16 \pi^{2}}\left(12 \lambda^{2}\right)+\left(\text { terms involving Yukawa couplings, } g, g^{\prime}\right)
$$


with $t=\log \frac{Q^{2}}{Q_{0}^{2}}$, where $Q$ is the renormalization scale and $Q_{0}$ is a reference scale, which is often taken to be equal to the vacuum expectation value $v$. Neglecting the terms with Yukawa couplings one can obtain the following solution to the equation 1.40:

$$
\frac{1}{\lambda\left(Q_{0}\right)}-\frac{1}{\lambda(Q)}=\frac{3}{4 \pi^{2}} \log \frac{Q^{2}}{Q_{0}^{2}}
$$

It can be shown [30] that the stability of the Higgs potential $d V(\phi) / d \phi>0$ is equivalent to the requirement $\lambda\left(Q_{0}\right) \geq 0$. Therefore, Eq. 1.41 can be written as:

$$
\lambda(\mu) \leq \frac{4 \pi^{2}}{3 \log \left(Q^{2} / \mu^{2}\right)}
$$

From Eq. 1.42 one can deduce that in the limit of large values of $Q^{2}$ the theory becomes trivial, i.e. $\lambda(\mu) \rightarrow 0$. If we assume that the new physics appears at a scale $\Lambda \sim M_{P l}$ and take the $\lambda(\Lambda)$ to have its maximum value $(\infty)$ and let the coupling evolve to the weak scale $v$, we find the maximum allowed value for the Higgs mass:

$$
\lambda(v)=\frac{m_{H}^{2}}{2 v^{2}} \leq \frac{4 \pi^{2}}{3 \log \frac{\Lambda^{2}}{v^{2}}}
$$

which yields a maximum scale for a given value $m_{H}$ of the Higgs boson mass. This bounds are represented by the upper curve in Fig. 1.3

\subsubsection{Experimental Bounds on the Higgs boson}

The experimental bounds on the Higgs boson can be classified in two categories: constraints from indirect measurements and those from direct searches. While the most definitive proof of the existence of the Higgs boson will come from the direct observation, the indirect bounds on its mass help in determining the direction of the searches. Since the Higgs boson enters in SM radiative corrections, the precision electroweak measurements allow to provide bounds on its mass. However, because of the Veltman's "screening theorem" [31] the electroweak corrections have a logarithmic dependence on $m_{H}$. In general, electroweak corrections involving the Higgs boson have the form:

$$
g^{2}\left(\log \frac{m_{H}}{M_{W}}+g^{2} \frac{m_{H}^{2}}{M_{W}^{2}}\right)
$$


Eq. 1.44 shows that the effects that are quadratic in $m_{H}$ are screened by an additional power of $g^{2}$. The LEP Electroweak Working Group has been performing global fits to the available precision data in order to determine the preferred value of the Higgs boson mass. Using various measurements from LEP, SLC, NuTeV and Tevatron experiments the fit suggests a preferred value of $m_{H}=84_{-26}^{+34} \mathrm{GeV}$ and an upper limit of $m_{H}<154 \mathrm{GeV}$ at $95 \%$ C.L. [32]. Fig. 1.4 shows the $\Delta \chi^{2}$ curve derived from high $Q^{2}$ data from precision electroweak measurements, performed by the LEP experiments and by SLD, CDF, and D0. Precision measurements of the top quark and $W$ boson masses also allow to constrain the allowed Higgs boson mass as summarized in Fig. 1.5.

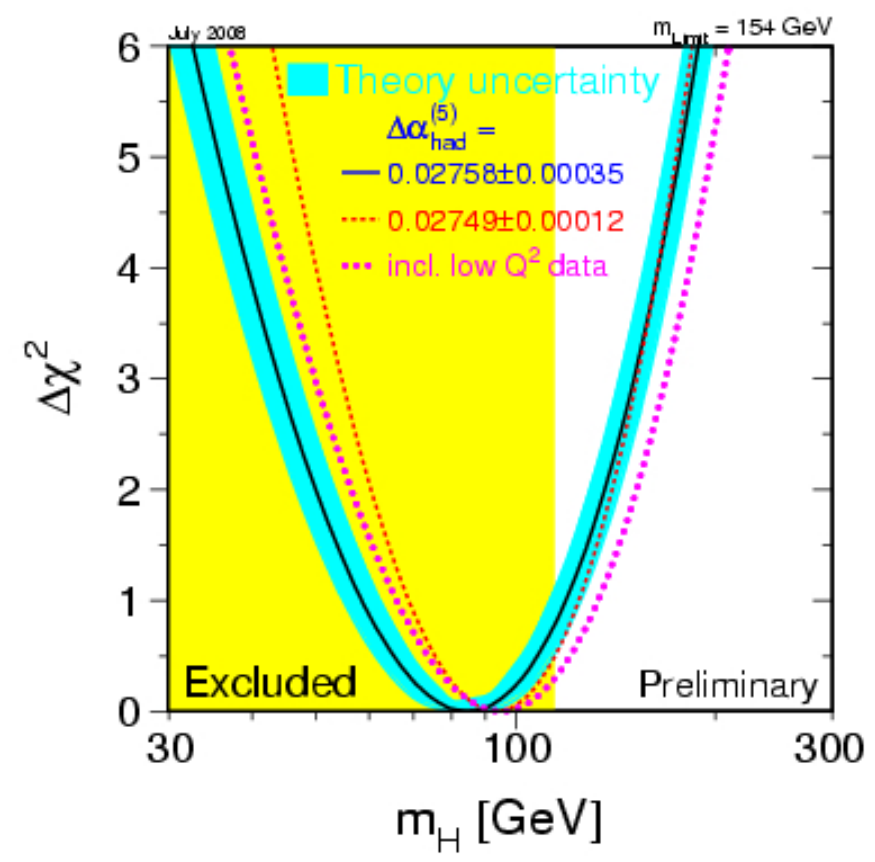

Figure 1.4.: $\Delta \chi^{2}$ of the global fit to the Standard Model as a function of the Higgs boson mass [32]

One of the main physics goals the LEP2, $e^{+} e^{-}$collider which operated at CERN from 1995 to 2000, was the search for the Higgs boson. The Standard Model Higgs 


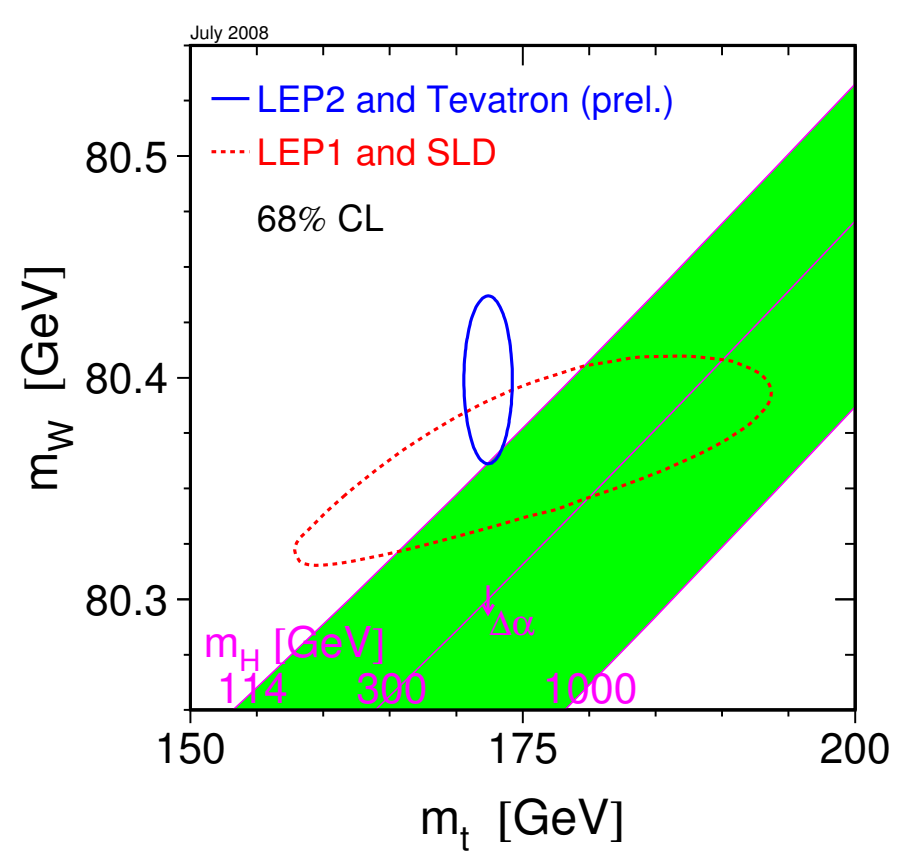

Figure 1.5.: Constraints on Higgs Mass from latest Tevatron measurement [33]

boson could be produced at LEP2 in the reaction $e^{+} e^{-} \rightarrow Z H$ which proceeds by $Z$ exchange. The non observation of the Higgs particle at LEP2 allowed to establish a lower limit on its mass: $m_{H} \gtrsim 114 \mathrm{GeV}$ [33], which is the most stringent bound on the Higgs boson mass to this date.

The direct searches for the Higgs boson at Tevatron are mainly driven by the production cross-section and the decay signatures at a given mass value. The Higgs boson production cross-sections and the branching fractions as a function of the Higgs mass are shown in Figures 1.6(a) and 1.6(b).

The main production mechanisms for the searches at Tevatron are:

- $g g \rightarrow H:$ gluon fusion is the process with the largest production cross-section. The cross section varies between 1.0-0.2 pb for $100 \mathrm{GeV} / c^{2}<m_{H}<200 \mathrm{GeV} / c^{2}$. 


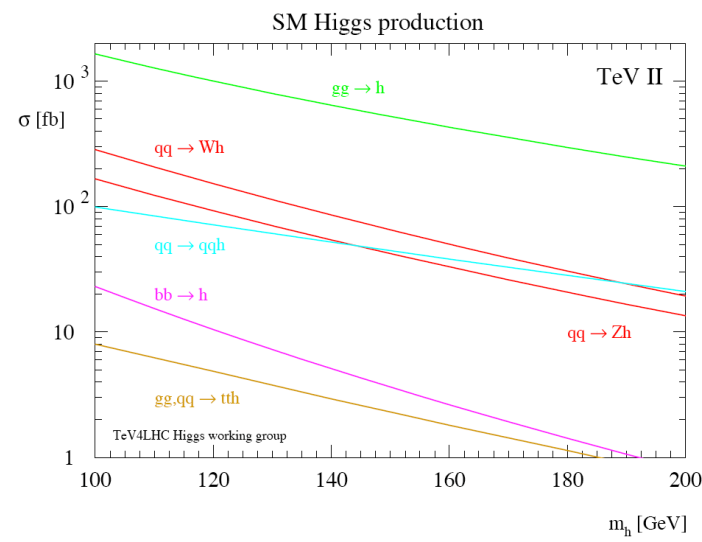

(a) SM Higgs boson production crosssections [34]

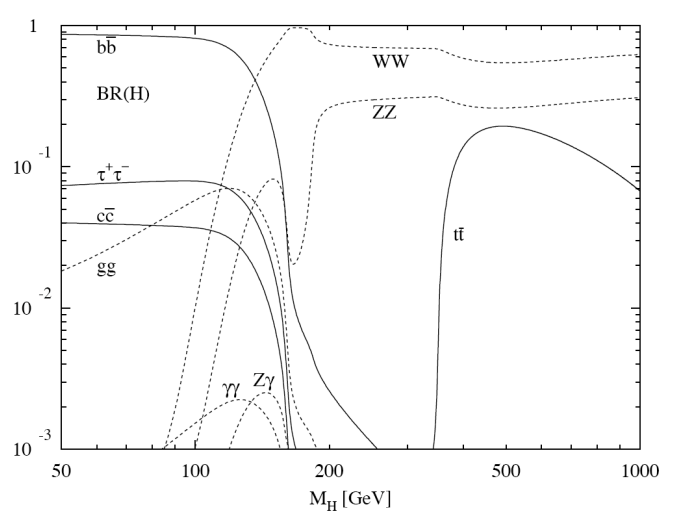

(b) SM Higgs boson branching ratios [35]

Figure 1.6.: Standard Model Higgs boson at the Tevatron

- $q q \rightarrow V H$ : associated vector boson production. The production cross-section in this channel is in the range $0.5-0.2 \mathrm{pb}$, if $100 \mathrm{GeV} / c^{2}<m_{H}<130 \mathrm{GeV} / c^{2}$.

While there are many other production mechanisms available at the Tevatron, the two mentioned above contribute the most. The searches for the Higgs boson are largely driven by possible decays of the Higgs boson and the ease of triggering on its decay products. Since the coupling of the Higgs boson to massive particles depends on their masses, the most frequent decays of the Higgs boson are to the heaviest particles.

If the Higgs boson is just above the LEP limit, i.e. $m_{H} \gtrsim 115 \mathrm{GeV}$, than the Higgs boson predominantly decays to $b \bar{b}$, as shown in Fig. 1.6(b). The $b$ quarks from the Higgs decay transform into hadrons and form collimated sprays of particles, called jets, which are identified in the detectors. The cross section of QCD production of quarks at hadron colliders is several orders of magnitude higher than $g g \rightarrow H$ , e.g. direct production of quark pairs or gluon splitting. Therefore, the searches in the range of $100 \mathrm{GeV}<m_{H}<130 \mathrm{GeV}$ the Higgs production is overwhelmed by large, essentially irreducible background from QCD multi-jet production. In the case of associated vector boson production, the decay products of the $W$ or $Z$ boson 
provide additional handles to reduce the amount of the backgrounds, and these are the production channels that are explored in the low mass searches.

If the Higgs boson mass is $m_{H} \gtrsim 130 \mathrm{GeV}$, the Higgs boson decays primarily to a pair of vector bosons. The decays of the $W$ and $Z$ bosons allow to highly suppress the backgrounds, and provide additional constraints through conservation of angular momentum, since the Higgs boson is a scalar particle. The searches in this mass range focus on the $g g \rightarrow H$ gluon fusion production with subsequent decays to charged leptons, which provide the highest signal yield.

Various search channels are then optimized for a particular final state, depending on the production mechanism and the decays of the produced particles. The main analysis channels at the Tevatron are the following:

1. If $100 \mathrm{GeV} / c^{2}<m_{H}<135 \mathrm{GeV} / c^{2}$ the searches focus on associated vector boson production

- $q q \rightarrow Z H \rightarrow l^{+} l^{-} b \bar{b}$ : this channel provides the cleanest signature, since the SM processes rarely produce a similar final state. Additionally, it is possible to fully reconstruct both $Z$ and $H$ bosons, allowing to further constrain the backgrounds. Traditionally the decays of a $Z$ boson to a pair of electrons or muons are considered, since the $\tau$ identification at hadron colliders is more challenging. Due to the low branching fraction $\left(B r\left[Z \rightarrow l^{+} l^{-}\right] \sim 0.07\right.$ for $e, \mu$ combined) the number of expected signal events in this channel is relatively low, with an expectation of around 6 events per $f b^{-1}$ at the Tevatron (if $m_{H}=115 \mathrm{GeV} / c^{2}$ )

- $q q \rightarrow W^{ \pm} H \rightarrow l^{ \pm} \nu b \bar{b}$ : due to the higher production cross-section compared to $Z$ associated production, and a higher branching fraction $\left(\operatorname{Br}\left[W^{ \pm} \rightarrow\right.\right.$ $\left.l^{ \pm} \nu\right] \sim 0.22$ for $e, \mu$ combined). This final state provides one of the most sensitive channels for Higgs boson searches. The expected number of Higgs boson events produced in this channel is around 31 per $f b^{-1}$ (if $m_{H}=$ $\left.115 \mathrm{GeV} / c^{2}\right)$. 
- $q q \rightarrow V H \rightarrow \not_{T} b \bar{b}$ : the main feature of this channel is the presence of a large energy imbalance in the transverse plane $\left(\mathscr{E}_{T}\right)$ and the absence of identified charged leptons from the decays of the vector bosons. The $\mathbb{E}_{T}$ in the events originates either from the $Z \rightarrow \nu \nu$ decays or from $W^{ \pm} \rightarrow l^{ \pm} \nu$ when the charged lepton escapes the detection. As a result, the effective production cross-section increases, but the lack of charged leptons weakens the constraints on the backgrounds. The expected number of Higgs boson events produced in this channel is around 32 per $f b^{-1}$ (if $\left.m_{H}=115 \mathrm{GeV} / c^{2}\right)$.

2. If $135 \mathrm{GeV} / c^{2}<m_{H}<200 \mathrm{GeV} / c^{2}$ the searches focus on the Higgs boson production through the gluon fusion:

- $g g \rightarrow H \rightarrow W^{+} W^{-} \rightarrow l^{+} l^{-} \nu \nu$ : searches in this channel benefit from large signal yields and the lack of large backgrounds with jets. Angular correlation between the leptons from the $W$ decays provide one of the most distinguishing feature of the signal events. The expected number of Higgs boson events produced in this channel is around 113 per $\mathrm{fb}^{-1}$ (if $m_{H}=170 \mathrm{GeV} / c^{2}$ in $e$ and $\mu$ channels combined).

The focus of this dissertation is on the search for the Higgs boson in the $q q \rightarrow$ $V H \rightarrow \not_{T} b \bar{b}$ channel in $2.1 \mathrm{fb}^{-1}$ of data collected by CDF experiment at Tevatron. This channel has an advantage of large amount of expected Higgs boson signal events, as described above. However, due to the final event signature, it suffers from contribution from many background sources, the most prominent of which is the QCD multi-jet production. Previous analysis in this channel used stringent event selections, to minimize the amount of QCD events with fake $\mathscr{E}_{T}$ passing into the search dataset, as a result of this strategy many expected signal events were removed. We devise a novel approach of removing the large backgrounds while keeping a large acceptance to signal events. Additionally, since a substantial contribution from QCD events still remains, we develop a new method to estimate this background from experimental 
data, without relying on simulation. These and other improvements in the analysis technique result in an improvement of a factor of 2 in sensitivity when compared with the latest CDF published result with $1 \mathrm{fb}^{-1}$, scaled to the same luminosity. 


\title{
Chapter 2. Experimental Apparatus
}

\author{
Measure what is measurable, and make measurable \\ what is not so.
}

Galileo Galilei (1564 - 1642)

\subsection{Accelerator Complex}

The Tevatron is a proton-antiproton superconducting collider at the Fermi National Accelerator Laboratory (FNAL) in Batavia, Illinois. It currently operates with 36 proton on 36 antiproton bunches at a center-of-mass energy of $\sqrt{s}=1.96 \mathrm{TeV}$ and a bunch spacing of $396 \mathrm{~ns}$. The bunches are equally divided into three $4.4 \mu \mathrm{s}$ bunch trains separated by $2.6 \mu$ s abort gaps. The bunch revolution time is approximately $21 \mu \mathrm{s}$. In collider operations the Tevatron takes $150 \mathrm{GeV}$ protons and antiprotons provided by the main injector and accelerates them to $980 \mathrm{GeV}$. Fig. 2.1 provides a schematic view of the Fermilab accelerator complex.

Proton and antiproton bunches circulate around the Tevatron in opposite directions. Their orbits cross at the B0 and D0 collision points, where interactions are observed by the CDF and D0 detectors respectively.

A large part of the physics program carried out by the CDF and D0 collaborations at the Tevatron involves searches for, and measurements of processes which have very small cross-sections compared to cross-sections of total inelastic collisions. In order to increase the significance of an observed signal the quantity $N_{\text {signal }} / N_{\text {background }}$ is

optimized, where $N_{\text {signal }}$ is the number of expected signal events and $N_{\text {background }}$ is the number of background events. These necessitates to collect as many $p \bar{p}$ collisions as possible. The measure of how many particle collisions are produced per unit time 


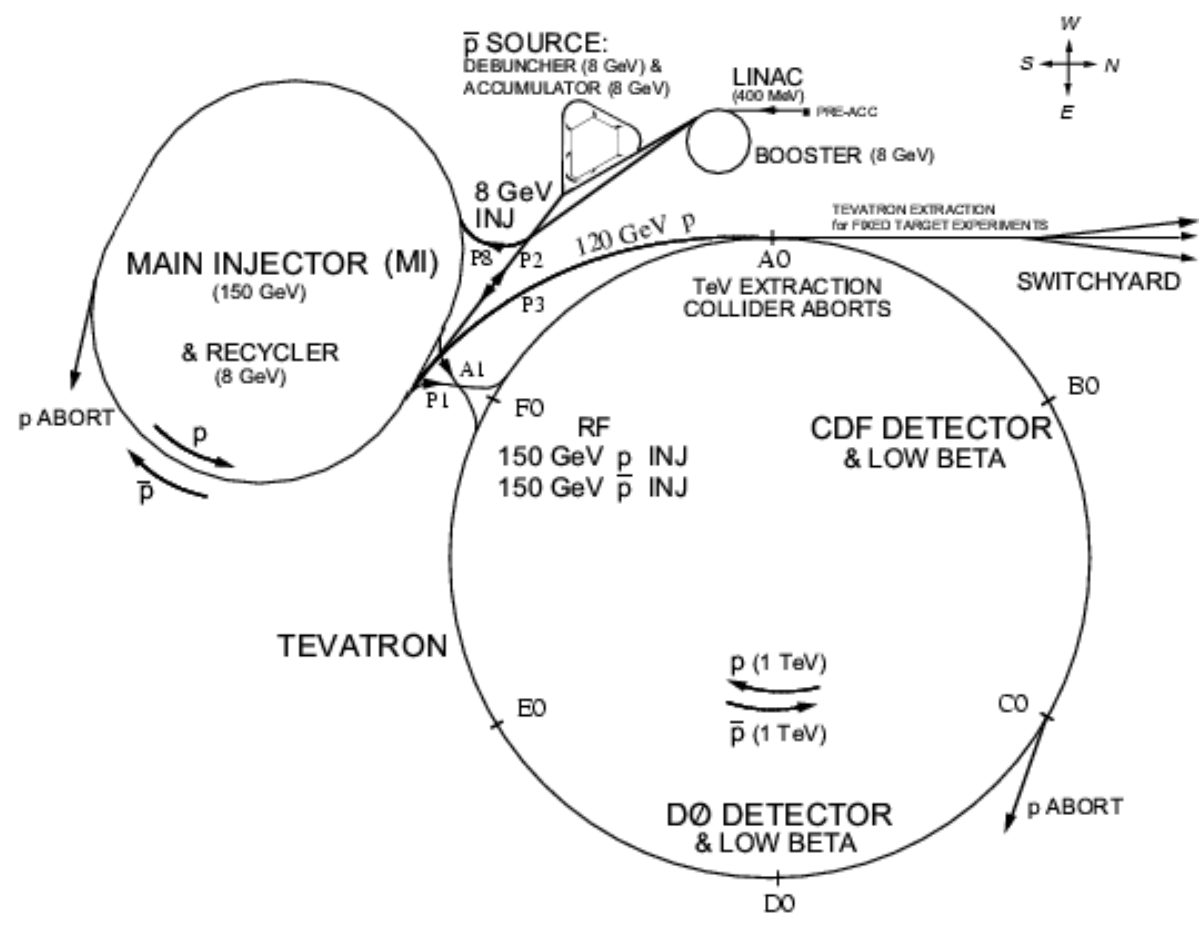

Figure 2.1.: A schematic view of the Teavtron

is referred to as the "instantaneous luminosity" $\mathcal{L}(t)$. The number $N_{\text {events }}$ due to a process with a cross section $\sigma$ is given by:

$$
N_{\text {events }}=\sigma \cdot \mathcal{L}_{\text {int }}
$$

where $\mathcal{L}_{\text {int }}$ is the integrated luminosity:

$$
\mathcal{L}_{\text {int }}=\int \mathcal{L}(t) d t
$$

The instantaneous luminosity is one of the key parameters defining an accelerator, and can be calculated using:

$$
\mathcal{L}=\frac{f B N_{p} N_{\bar{p}}}{2 \pi\left(\sigma_{p}^{2}+\sigma_{\bar{p}}^{2}\right)} F\left(\frac{\sigma_{l}}{\beta^{*}}\right)
$$

where $f$ is the revolution frequency, $B$ is the number of bunches in each beam, $N_{p}$ $\left(N_{\bar{p}}\right)$ is the number of protons (antiprotons) in a bunch, $\sigma_{p}\left(\sigma_{\bar{p}}\right)$ is the rms proton (antiproton) beam size at the interaction point, and $F$ is a form factor that depends 
on the ratio of the bunch length, $\sigma_{l}$, to the beta function at the interaction point $\beta^{*}[36]$. The beta function is a measure of the beam width, and is proportional to the beam's $x$ and $y$ spread in phase space. The parameters of the Tevatron accelerator are summarized in the Tab.

Table 2.1: Characteristic parameters of the Tevatron accelerator

\begin{tabular}{|c|c|}
\hline Parameter & Value \\
\hline energy at the center-of-mass & $1.96 \mathrm{TeV}$ \\
number of bunches & 36 \\
bunch length (rms) & $0.37 \mathrm{~m}$ \\
bunch spacing & $396 \mathrm{nsec}$ \\
abort gap & $2.6 \mu \mathrm{s}$ \\
protons per bunch & $2.7 \cdot 10^{11}$ \\
antiprotons per bunch & $3.0 \cdot 10^{10}$ \\
antiproton production rate & $2.0 \cdot 10^{11} \mathrm{hr}^{-1}$ \\
$\beta^{*}$ & $35 \mathrm{~cm}^{*}$ \\
\hline
\end{tabular}

The unit adopted to measure the cross-sections in high energy collisions is the "barn" b, and is equivalent to $10^{-24} \mathrm{~cm}^{2}$. Typical values of cross-sections of processes observed at particle accelerators are fractions of a barn, such as $\mathrm{pb}=10^{-12} \mathrm{~b}$ or $\mathrm{fb}=10^{-15} \mathrm{~b}$. For example, the $b \bar{b}$ production cross-section is of the order of a few $\mu \mathrm{b}$ and the $t \bar{t}$ production cross-section is $\sim 7 \mathrm{pb}$. The integrated luminosity is measured in units of $b^{-1}$.

\subsubsection{The Linear Accelerator and Booster}

A schematic overview of the accelerator chain at Fermilab is given in Fig. 2.1. The acceleration occurs in a number of stages and system of succeeding accelerators is utilized in order to generate and accelerate the protons and antiprotons that collide 
with a center-of-mass energy $\sqrt{s}=1.96 \mathrm{TeV}$. The Cockcroft-Walton pre-accelerator provides the first stage of acceleration. Inside this device, hydrogen gas is ionized to create negative ions $H^{-}$. The hydrogen ions are accelerated to $750 \mathrm{keV}$ kinetic energy in the Cockcroft-Walton pre-accelerator. Those preaccelerated ions are passed to the LINAC (LINear ACcelerator) where they are accelerated to $400 \mathrm{MeV}$ by radio frequency $(\mathrm{RF})$ resonators. At the end of the LINAC, after about $130 \mathrm{~m}$, the negative hydrogen ions enter the first synchrotron in the acceleration chain, the Booster with a circumference of about $475 \mathrm{~m}$, after passing a carbon foil where the electrons are stripped off. In the Booster, the remaining protons are grouped into 84 bunches, each containing around $6 \cdot 10^{6}$ protons, and are accelerated to $8 \mathrm{GeV}$ before entering another synchrotron: the Main Injector.

\subsubsection{The Antiproton Source}

The Antiproton Source is made up of three parts: the nickel target, the Debuncher Ring and the Accumulator. For antiproton production, or "stacking" as it is called, one proton batch from the Booster is injected into the Main Injector and accelerated to $120 \mathrm{GeV}$. The proton beam is extracted from the Main Injector and sent to the Antiproton Source where it hits a nickel target, producing a spray of particles including antiprotons. Antiprotons with energies of approximately $8 \mathrm{GeV}$ are collected out of this spray by a lithium lens and sent to the Debuncher where the large spread in energy of the antiprotons is reduced. This is achieved by transforming the beam with a large energy spread and a narrow time distribution (i.e., a bunched beam) into a beam with a narrow energy spread and a large time distribution (i.e., a continuous beam). As a result, the bunch structure of the beam is lost. The antiproton bunches are collected and stacked in the Accumulator ring, where they are cooled down and the bunch structure of the beam is recovered. 


\subsubsection{The Recycler Ring}

A limiting factor in producing high luminosities at the Tevatron is the number of antiprotons available. The purpose of the Recycler Ring (RR) is to store excess antiprotons. The RR stores antiprotons from the Antiproton Source and cools them further than the Accumulator can achieve. The Main Injector transfers antiprotons from the Accumulator to the RR, which has its own RF system to facilitate transfers to and from the Main Injector. When needed, the RR sends its antiprotons to the Main Injector. The RR can hold up to $5 \cdot 10^{12}$ antiprotons.

\subsubsection{The Main Injector}

The Main Injector (MI) is a circular synchrotron which has a circumference of $3319 \mathrm{~m}$. It accelerates $8 \mathrm{GeV}$ protons from the Booster to either $120 \mathrm{GeV}$ or $150 \mathrm{GeV}$, depending on their destination. When used for stacking, the final energy is $120 \mathrm{GeV}$, while when used to inject into the Tevatron, the final beam energy is $150 \mathrm{GeV}$. The most complex mode of the Tevatron operations is the "Collider Mode", and the MI plays various roles in this mode. The MI receives about 7 of 84 proton bunches from the Booster and accelerates them to $150 \mathrm{GeV}$. These bunches are "coalesced" (pushed together to form a narrow, high intensity bunch), and injected to the Tevatron continuously, until 36 coalesced proton bunches are transferred. Once all of the protons are transferred to the Tevatron, four groups of antiprotons leave the Accumulator and enter the Main Injector and circulate clockwise, in the opposite direction of the protons. The four antiproton groups are then accelerated to $150 \mathrm{GeV}$ and injected to the Tevatron. This process repeats until there are 36 coalesced bunches of antiprotons and protons circulating in the Tevatron at $150 \mathrm{GeV}$. After the process of injection of protons and antiprotons is finished the MI returns to stacking. 


\subsubsection{The Tevatron}

The Tevatron is the largest of the Fermilab accelerators, with a circumference of approximately $6 \mathrm{~km}$ [37]. It is the worlds first superconducting synchrotron and is currently the highest energy operating accelerator in the world. The Tevatron accepts protons and antiprotons from Main Injector and accelerates them from 150 $\mathrm{GeV}$ to $980 \mathrm{GeV}$. It uses cryogenically cooled magnets, made of niobium/titanium alloy kept at $T \approx 4 \mathrm{~K}$, to accelerate the beam. The proton and antiproton beams are circulated in opposite directions in the Tevatron, and are split into three trains, each containing 12 bunches with 396 ns separation. Empty spaces between the trains are called "abort gaps", and allow time for the kicker magnets to ramp up. The kicker magnets are used to abort the beam by guiding it into graphite beam dumps. There are two points at points B0 and D0 (Fig. 2.1) where the two beams are crossed and proton-antiproton collisions can occur. The products of the collisions are recorded by CDF and DØ detectors.

\subsection{The CDF II detector}

The Collider Detector at Fermilab (CDF II) [38] shown in Fig. 2.2 is a multipurpose experiment with azimuthal and forward-backward symmetry, designed to study high energy $p \bar{p}$ collisions. It combines precision charged particle tracking with projective calorimetry and fine grained muon detection. The tracking system is contained inside a superconducting solenoid of $4.8 \mathrm{~m}$ length and $1.5 \mathrm{~m}$ radius that generates a 1.4 T magnetic field parallel to the beam axis. The magnetic field is uniform with an accuracy of $0.1 \%$ throughout the entire tracking volume. The muon and calorimetry systems are located outside the solenoid. 
CDF uses a coordinate system with the positive z-axis lies along the direction of the incident proton beam, $\phi$ is the azimuthal angle, $\theta$ is the polar angle (measured from the detector center). The rapidity, $y$, of a particle is given by

$$
y=\frac{1}{2} \ln \left(\frac{E+p_{z}}{E-p_{z}}\right) .
$$

Rapidity differences are useful in physics analysis, since they are invariant to a Lorentz boost. The pseudo-rapidity, $\eta$, is defined as $\eta=-\ln \left(\tan \frac{\theta}{2}\right)$. The pseudorapidity $\eta$ equals the rapidity $y$ in the massless approximation $\left(E \gg m c^{2}\right)$.

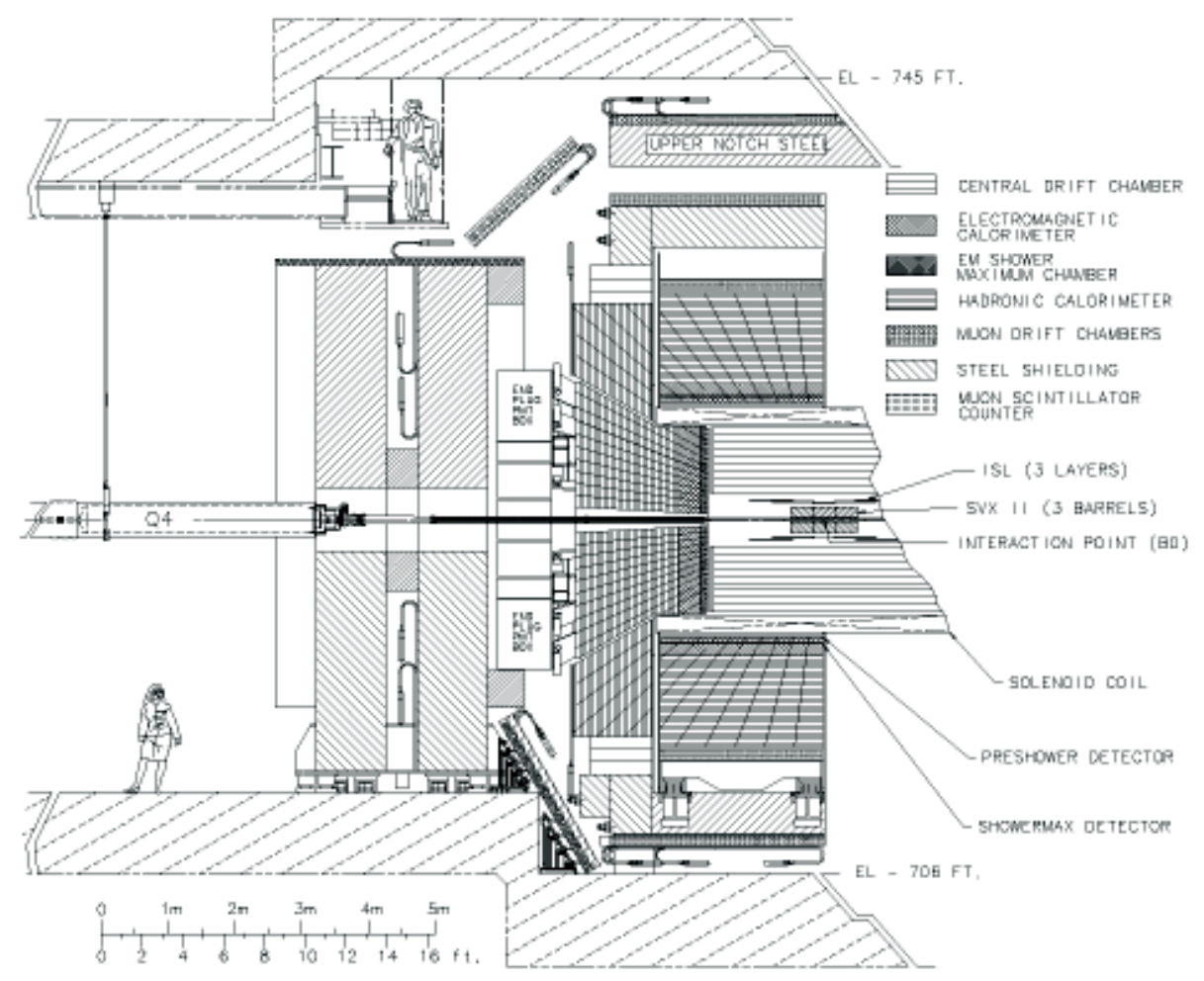

Figure 2.2.: A cross-sectional view of one half of the CDF II detector. 


\subsubsection{Tracking System}

The tracking system is used to reconstruct the trajectories of charged particles: "tracks" and precisely measure their momenta.

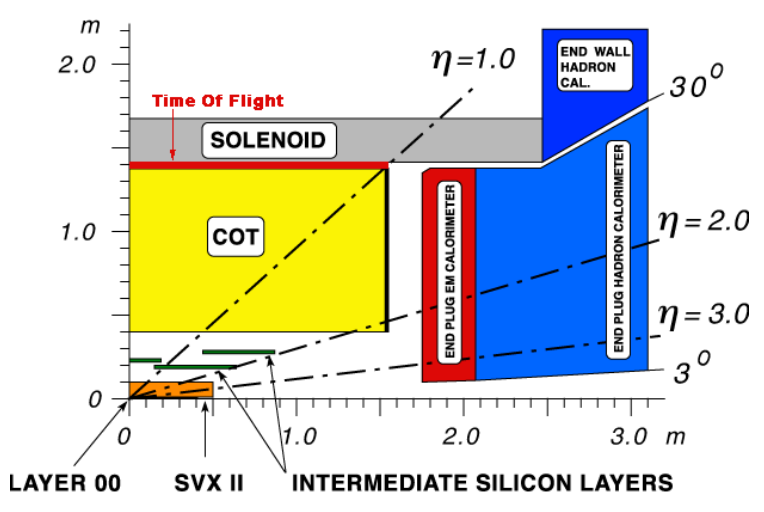

(a)

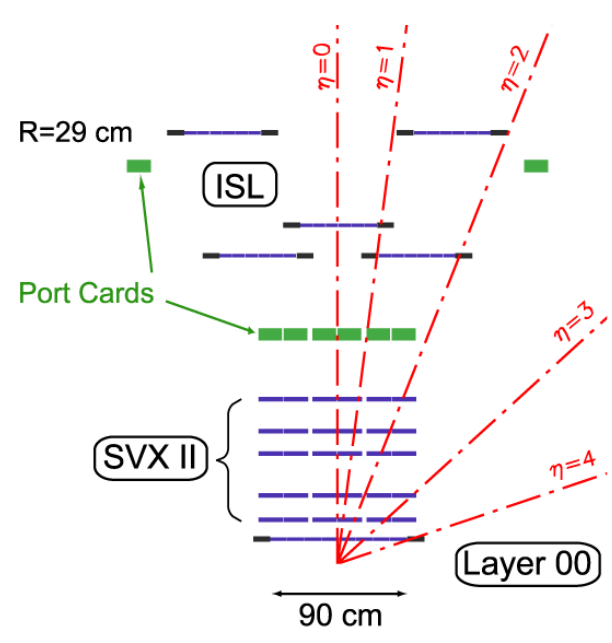

(b)

Figure 2.3.: A view of the CDF tracking system: (a) cross section view of the CDF II tracking system, (b) the $r-z$ view of the CDF II silicon system

The tracking system is installed close to the beam pipe and is composed of an "inner" and "outer" tracker (Fig. ??). The inner tracker relies on a silicon strip detector comprised of three sub-detectors:

- L00 (Layer 00) [39] is a radiation-hard single-sided detector and is the first detector particles encounter after leaving the beam pipe, since the L00 detector is directly attached to the beam pipe. The silicon sensors of L00 are arranged in two overlapping sublayers at $r=1.35 \mathrm{~cm}$ and $r=1.62 \mathrm{~cm}$ (Fig. 2.4(a)), covering $|\eta| \leq 4$.0. While the L00 provides information only in the $r-\phi$ plane, it provides an important improvement the impact parameter resolution, as shown in Fig. 2.4(b). L00 was designed to recover degraded impact parameter resolution due to multiple scattering off passive material in SVX II. 


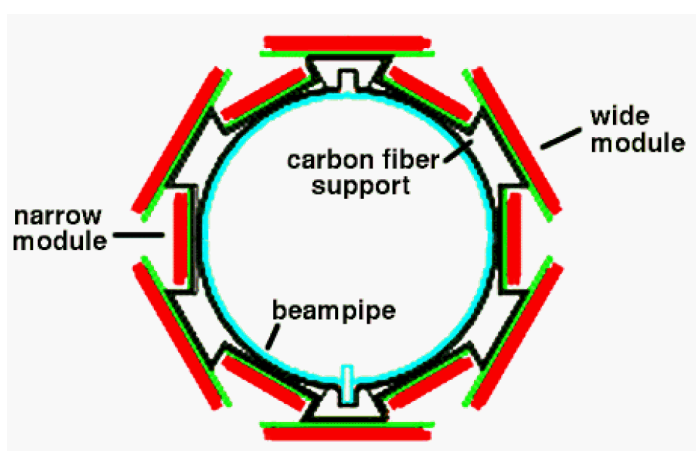

(a)

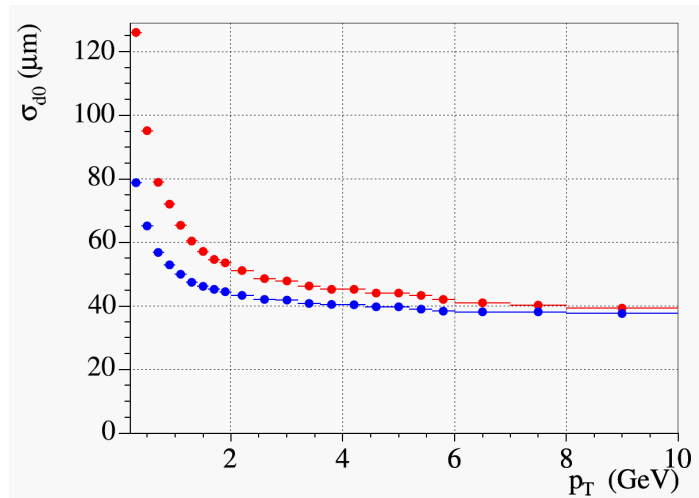

(b)

Figure 2.4.: The L00 detector: (a) a schematic overview of the L00 detector and (b) the impact parameter resolution of tracks with L00 hits (red) and without L00 hits (blue)

- Silicon Vertex Detector (SVX II) $[40,41]$ is built in three cylindrical barrels of five layers of double-sided microstrip detectors (Fig. 2.5(a)). One side of the detectors measure the $\phi$ position and the other side in $z$. The strips on one side of all detectors run along the $z$-axis ( "axial" strips), while on the other side the strips ( "stereo" strips) are perpendicular to the $z$-axis in three layers and tilted by $1.2^{\circ}$ with respect to the transverse direction on the other two (layers 2 and 4 in Fig. 2.5(b)). This design allows for a three-dimensional reconstruction of the tracks of the charged particles.

- The last element of the silicon inner tracker is the Intermediate Silicon Layer (ISL) [42], which links the inner tracking region and the outer tracker. The $1.9 \mathrm{~m}$ long ISL consists of three layers: a central layer at $r=22 \mathrm{~cm}$ and $|\eta<| 1$, and two forward layers at $r=20$ and $28 \mathrm{~cm}$ and $1<|\eta|<2$, as shown in Fig. 2.6(b). The silicon sensors of ISL are double-sided small angle stereo $\left(1.2^{\circ}\right)$ ladders which measure the positions of the hist in both $r-\phi$ and $z$ directions. 


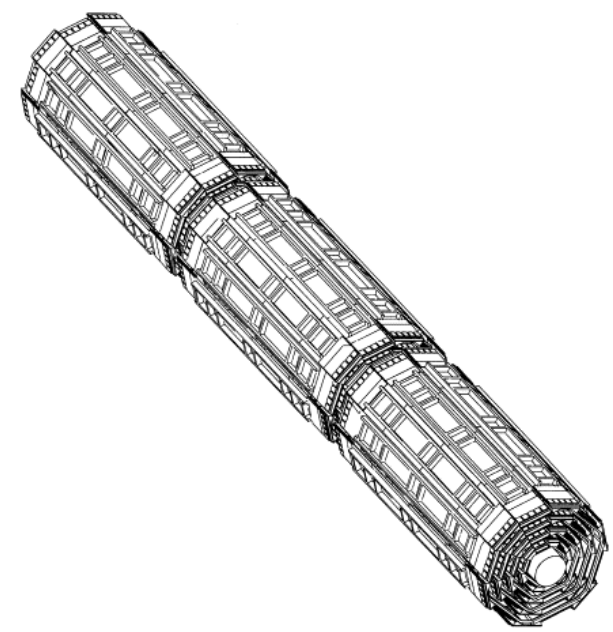

(a)

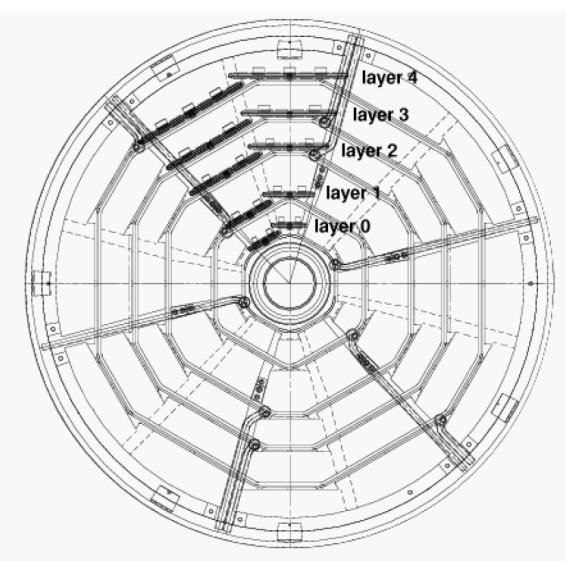

(b)

Figure 2.5.: The SVX II detector: (a) the view of the three SVXII barrels, (b) a schematic overview of the SVX components

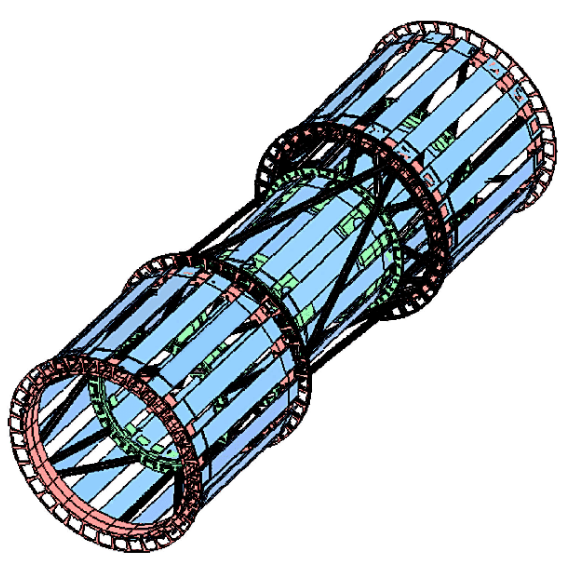

(a)

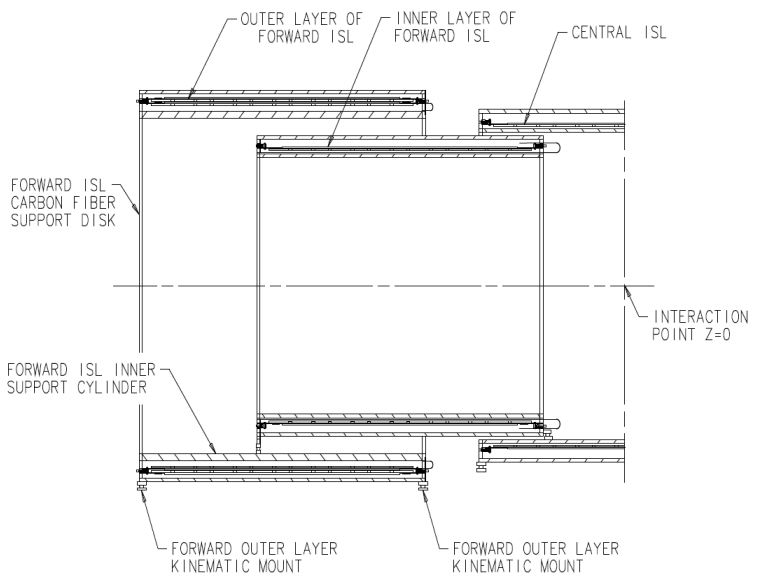

(b)

Figure 2.6.: The ISL detector: (a) a perspective view of the ISL space frame and silicon placement, (b) a schematic overview of the ISL components

\subsubsection{Central Outer Tracker}

The Central Outer Tracker (COT) [43] is a cylindrical open-cell drift chamber, that is located between the inner silicon tracker and the Time Of Flight detector, in the radial range from $40 \mathrm{~cm}$ to $138 \mathrm{~cm}$ (Fig. 2.3(a)). In $z$ it extends from $-155 \mathrm{~cm}$ to 
$+155 \mathrm{~cm}$, providing full coverage for $|\eta|<1$ and has a maximum acceptance of $|\eta|<$ 2.0 .

The COT contains 96 sense wire layers in radius that are grouped into 8 "superlayers", as shown in Fig. 2.7(a). Each superlayer is divided in $\phi$ into "supercells", and each supercell has 12 sense wires. Each sense wire has a potential wire on both sides to form the drift cell, as seen in Fig. 2.7(b), and the nominal spacing between sense and potential wires is around $0.36 \mathrm{~cm}$. Alternating layers have the wires either

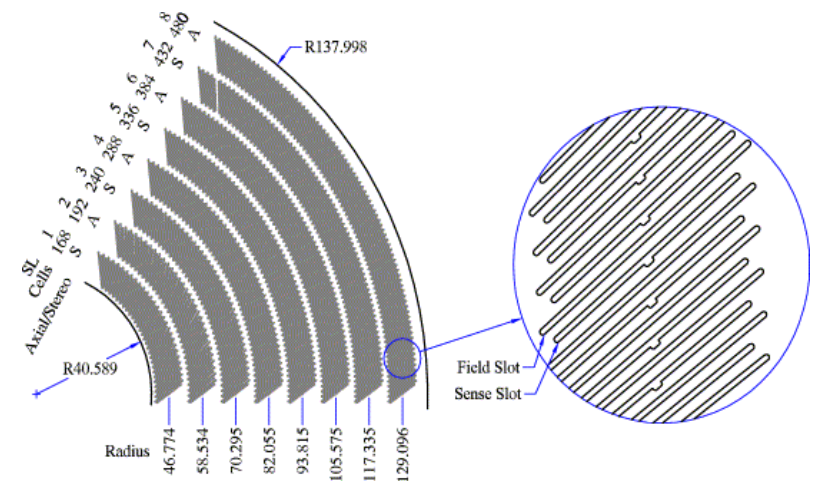

(a)

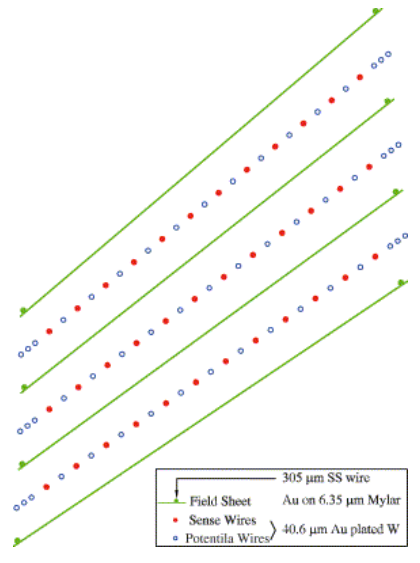

(b)

Figure 2.7.: The COT detector: (a) 1/6 section of the COT end plate. For each superlayer the total number of supercells, the wire orientation (axial or stereo), and the average radius is given. (b) Three supercells looking along the beam $z$ direction.

in the axial direction (along the $z$ axis), or at a small stereo angle $\left( \pm 2^{\circ}\right)$. The axial layers provide tracking information in $r-z$ plane and the stereo layers in the $z$ direction. The voltages on the sense wires are 2600-3000 Volts and 1000-2000 Volts on the potential wires. The chambers are filled with a 50:50 mixture of Argon-Ethane gas bubbled through isopropyl alcohol (1.7\%).

As charged particles travel through the COT, they ionize the gas mixture. Electrons created in the ionization, drift towards the sense wires, creating an avalanche of electrons through secondary ionization. The velocity of the drift in the Argon-Ethane mixture is $\sim 50 \mu \mathrm{m} / \mathrm{s}$, and the hit signals are collected in less than $200 \mathrm{~ns}$. Since the 
COT detector in enclosed in the $1.4 \mathrm{~T}$ magnetic field, the drifting electrons experience a Lorentz force that rotates their path. To compensate for this effect, the supercells are tilted by $35^{\circ}$ with respect to the radial direction, which equals the Lorentz angle in the COT. As a result, the electron drift is approximately perpendicular to the wires. The hit position resolution in the COT is approximately $140 \mu \mathrm{m}$, and the momentum resolution $\sigma\left(p_{T}\right) / p_{T}^{2}=0.0015(\mathrm{GeV} / \mathrm{c})^{-1}$. Additionally, the COT provides important information for particle identification. Measurements of the ionization per unit track length, $d E / d x$, which are characteristic of particle velocity, can be used to separate kaons, pions and protons. The separating power between kaons and pions with $p_{T}>2 \mathrm{GeV} / \mathrm{c}$ is 1.4 standard deviations.

\subsubsection{Time of Flight Detector}

As mentioned above, the ionization energy loss measured through $d E / d x$ in the COT, can be used for particle identification. However, the measurement from COT provides about one standard deviation separation between charged kaons and pions for momenta greater than $2 \mathrm{GeV} / c$. The goal of the Time Of Flight (TOF) detector [44] is to complement the measurement from the COT by distinguishing $K^{ \pm}$and $\pi^{ \pm}$in the region of cross-over in $d E / d x$.

The TOF is located between the COT and the CDF solenoid at a radius of $140 \mathrm{~cm}$ and provides coverage in $|\eta| \leq 1$, as shown in Fig 2.3(a). It consists of 216 scintillator bars that measure the arrival time $t_{f l i g h t}$ of a charged particle with respect to the collision time. In combination with the precise measurement of momentum $p$ and path length $L$ from the tracking system, the TOF detector provides particle identification by determining the particles mass using the formula:

$$
m=\frac{p}{c} \sqrt{\frac{c^{2} t_{\text {flight }}^{2}}{L^{2}}-1} .
$$

The TOF system achieves a resolution of $100 \mathrm{ps}$, which allows for at least two standard deviation separation between $\mathrm{K}^{ \pm}$and $\pi^{ \pm}$for momenta $\mathrm{p}<1.6 \mathrm{GeV} / c$, Fig. 2.8, complementing the $d E / d x$ measurement from COT. 


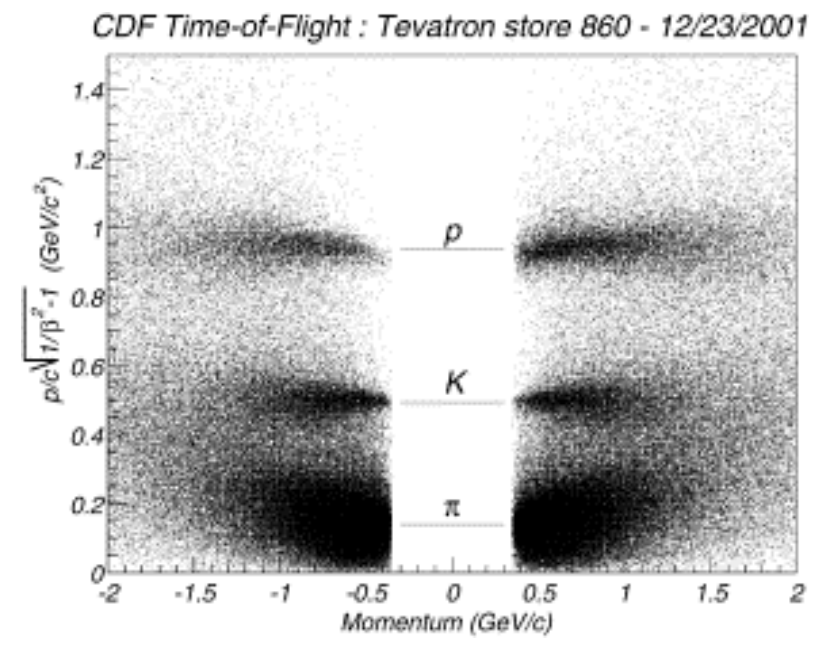

Figure 2.8.: Mass distribution from TOF measurement versus momentum for positive and negative tracks. The three horizontal lines correspond to nominal $p, K$, and $\pi$ masses.

\subsubsection{Superconducting Solenoid}

The entire CDF tracking system and the TOF detector are contained inside a $3 \mathrm{~m}$ diameter $\times 5 \mathrm{~m}$ superconducting solenoid [45]. An aluminum-stabilized $\mathrm{NbTi} / \mathrm{Cu}$ superconductor is used, which is cooled with liquid helium. The solenoid operates with a current of $\sim 4650$ A providing a magnetic field of $1.4 \mathrm{~T}$, which points in the $z$ direction. Since various detector elements, such as the calorimeters and muon detectors, are placed radially outside the solenoid, the solenoid was required to be thin in terms of radiation and absorption lengths in order to minimize absorption and scattering of particles passing through it. The overall material thickness of the solenoid is 0.85 radiation length in radial direction.

\subsubsection{Calorimetry System}

Scintillator-based calorimeters, located outside of the solenoid, allow the measurement of the particle and jet energies by absorbing all charged and neutral particles, 
except muons and neutrinos. Energies of the particles are measured through the showers that they produce, while transversing the detector volume. The calorimeters can also be used for measuring transverse energy of weakly interacting particles, such as neutrinos that escape detection, by computing the imbalance in the total transverse energy.

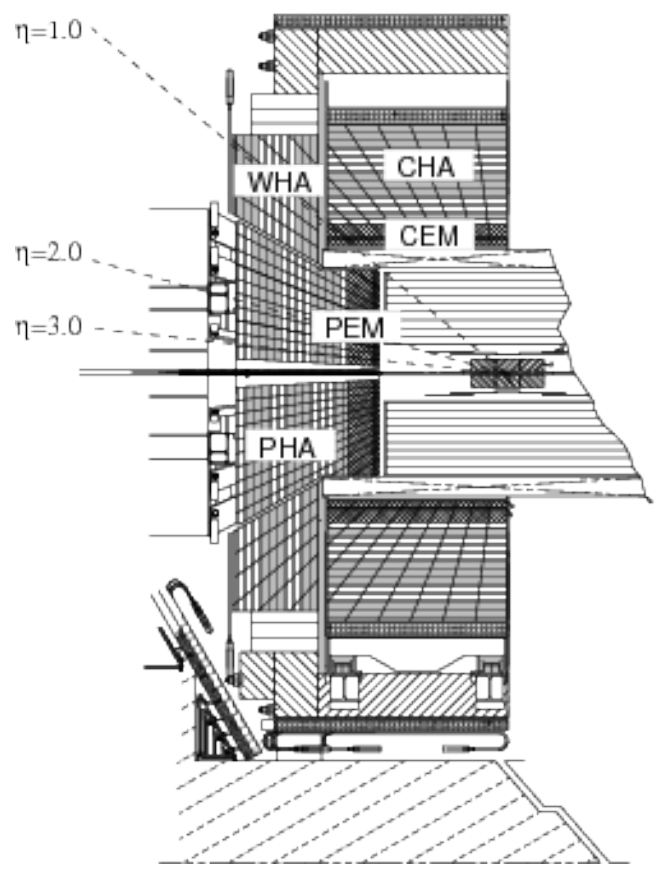

(a)

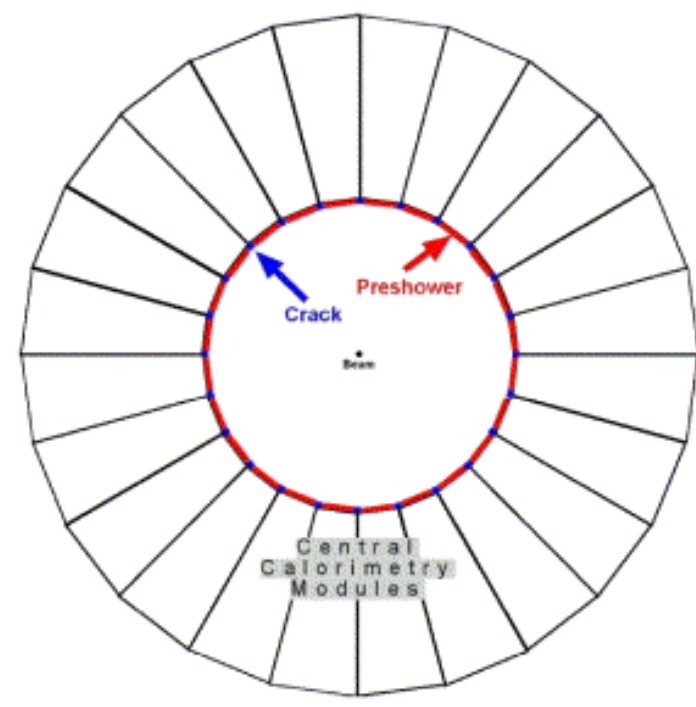

(b)

Figure 2.9.: (a) elevation view of one half of the CDF detector displaying the components of the CDF calorimeter, (b) the central calorimetry wedges and the location of the preshower and crack detectors.

The calorimeters are designed to be thick enough to fully capture all of the energy that the particle possesses. CDF uses sampling calorimeters, which are divided into an inner electromagnetic and an outer hadronic calorimeters. Both calorimeters are segmented into projective towers, consisting of alternating layers of passive absorber (made from a heavy element) and active scintillator tiles for shower sampling. The coverage of the calorimeters is nearly $4 \pi$ solid angle, and covers all of $\phi$ and the region 
$|\eta|<3.6$. The summary of the main characteristics of the various parts of the CDF calorimeters is shown in Tab. 2.2.

Table 2.2: Main characteristics of the EM and HAD calorimeters of CDF.

\begin{tabular}{|c|c|c|c|}
\hline Calorimeter & $\eta$ coverage & Depth & Resolution \\
\hline CEM & $|\eta| \leq 1$ & $18 X_{0}$ & $13.5 \% / \sqrt{E} \oplus 1.5 \%$ \\
PEM & $1.1 \leq|\eta| \leq 3.6$ & $23.2 X_{0}$ & $16 \% / \sqrt{E} \oplus 1 \%$ \\
CHA & $|\eta| \leq 0.9$ & $4.7 \lambda_{0}$ & $50 \% / \sqrt{E} \oplus 3 \%$ \\
WHA & $0.9 \leq|\eta| \leq 1.3$ & $4.7 \lambda_{0}$ & $75 \% / \sqrt{E} \oplus 4 \%$ \\
PHA & $1.3 \leq|\eta| \leq 3.6$ & $6.8 \lambda_{0}$ & $80 \% / \sqrt{E} \oplus 5 \%$ \\
\hline
\end{tabular}

The amount of energy lost by a particle passing through the calorimeter depends on the particle type and its initial energy. Energies of photons and electrons are measured by the electromagnetic calorimeter [46]. Electrons passing through the detector material lose their energy mostly through bremsstrahlung, while the photons produce electron-positron pairs. A cascade of these two processes forms a shower in the detector. As the particles transverse the detector volume, the amount of energy deposited by the showers increases until reaches a maximum depth, at which point the particles in the shower are no more enough energetic for further particle multiplication. This is referred to as the "shower maximum". The Central Electromagnetic Showermax (CES [47]) and the Plug Electromagnetic Showermax (PES [48]) detectors are used for photon and electron identification, and are located at around $6 X_{0}$ distance into the detector. The distance over which a traversing electron loses on average $1-e^{-1}$ fraction of its energy in a given material is called radiation length $X_{0}$, and is of the main characteristics of the detector.

The hadronic calorimeter [49] is used to measure the energies of charged and neutral hadrons, since the energy losses due to bremsstrahlung for most charged particles, except for electrons are negligible. Hadrons can lose their energy by ionization or secondary nuclear interactions, forming showers similar to electrons and photons. 
The mean free path necessary for a hadron to undergo a nuclear inelastic interaction is referred to as the nuclear interaction length, $\lambda_{0}$. Due to a higher multiplicity in nuclear interactions, the number of interaction lengths needed for the hadrons to lose their energy is fewer than for electrons or photons, although the interaction length $\lambda_{0}$ is usually larger than the radiation length $X_{0}$.

Overall, the CDF calorimeter system consists of Central Electromagnetic and Hadronic Calorimeters (CEM and CHA), Plug Electromagnetic and Wall Hadronic Calorimeters (PEM and WHA) and the Plug Hadronic Calorimeter (PHA), as shown in Fig. 2.9.

\section{Central Calorimeters}

The central calorimeters CEM, CHA and WHA are inherited from CDF Run I and cover the region $|\eta|<1$.1, divided into two halves along the $z$ direction at $|\eta|=0$ where there is a small uninstrumented region ( "crack"). The CEM consists of 478 towers, each of which measures $\Delta \eta \times \Delta \phi=0.1 \times 15^{\circ}$ in the $\eta-\phi$ plane. Two locations where there could have been colorimetric towers installed, were left uninstrumented to allow for space for the cryogenic system of the solenoid. The CEM consists of 31 layers of $5 \mathrm{~mm}$ thick polystyrene scintillator as the active medium, interspersed with $3.2 \mathrm{~mm}$ thick lead.

The Central Hadronic Calorimeter (CHA) is constructed with 32 layers of $2.5 \mathrm{~cm}$ thick steel alternated with $10 \mathrm{~mm}$ thick layers of scintillator. The Wall Hadronic Calorimeter contains only 15 layers of $5.1 \mathrm{~cm}$ thick absorber, which is why the energy resolution of the WHA detector is worse, as seen in Tab. 2.2.

\section{Plug Calorimeters}

The plug calorimeters PEM and PHA extend the CDF detector's coverage to $|\eta|<3.6$, and were a major part of Run II upgrade at CDF. The PEM is composed of 23 layers and 12 towers in $\eta$. The four most forward towers in $\eta$ are segmented 
in $15^{\circ}$, similar to the central detectors, while the remaining 8 towers have finer $\phi$ segmentation of $7.5^{\circ}$. The PEM detector consists of 23 layers of $4.5 \mathrm{~mm}$ of lead, interspersed with $4 \mathrm{~mm}$ thick scintillator. The PHA contains 23 layers of $6 \mathrm{~mm}$ of scintillator, alternated with $50 \mathrm{~mm}$ of iron.

\subsubsection{Muon Detector}

Muons are able to pass through large amounts of material before losing a significant amount of energy. The reason is that muons are minimum ionizing particles in a large energy range, from a few hundred $\mathrm{MeV}$ to several $\mathrm{TeV}$, and lose much less energy in bremsstrahlung than electrons. In contrast to muons, the majority of particles produced in $p \bar{p}$ collisions are absorbed by the calorimeter material. Hence, the muon system is the outermost layer of the CDF detector. To purify the muon sample, additional $60 \mathrm{~cm}$ of steel absorber is placed immediately after the calorimeter and the magnet return yoke. Four independent systems of scintillators and wire chambers, filled with a 50:50 ratio of Argon-Ethane mixture, are used for muon detection at $\mathrm{CDF}$, and provide muon coverage in $|\eta|<1.5$, as shown in Fig. 2.10(a). The chambers are stacked in 4 layers in the radial direction, and are coupled to scintillators (Fig. 2.10(b)). Hits in three matching layers are referred to as a "muon stub". Muon stubs that match to the extrapolation of a COT track constitute a muon candidate.

The four muon detectors at CDF are the following:

- Central MUon detector (CMU)

- Central Muon uPgrade (CMP)

- Central Muon eXtension (CMX)

- Barrel MUon detector (BMU)

The CMU detector [50] consists of 144 modules with 16 rectangular cells per module, covering the $|\eta| \leq 0.6$ region and providing the measurement of the $\phi$ and 


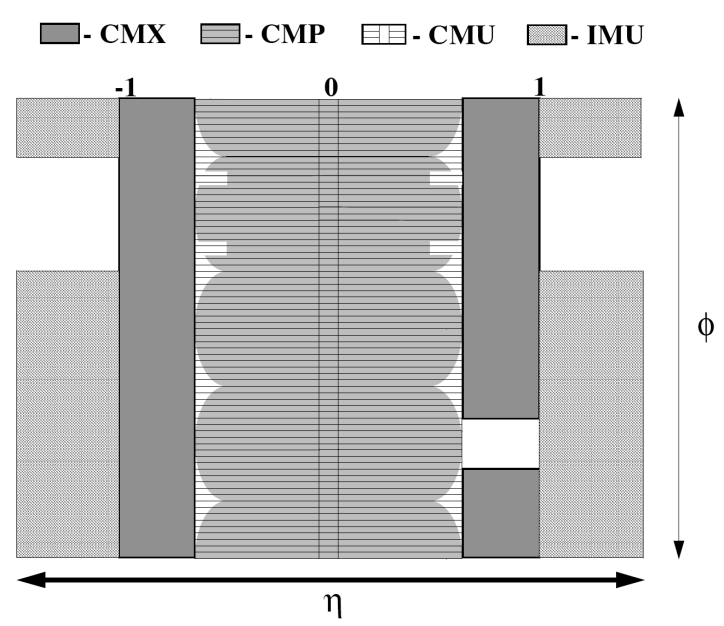

(a)

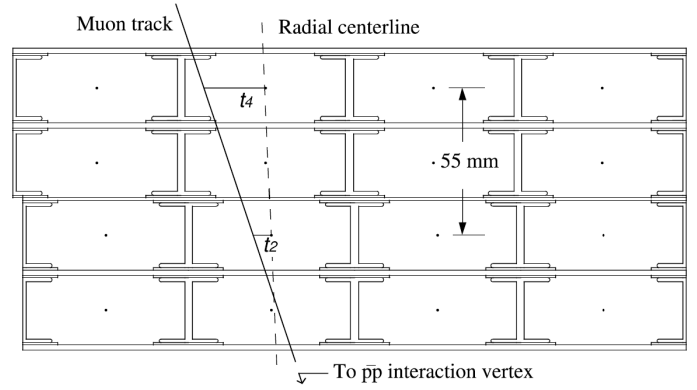

(b)

Figure 2.10.: Muon detectors in CDF: (a) map of the muon coverage as a function of azimuthal angle $\phi$ and pseudorapidity $\eta$, (b) transverse view of the CMU, showing the four layers in the radial direction

$z$ coordinates of the muon candidates. The CMU is installed at a distance of $5.5 \lambda_{0}$ from the interaction point. Wires in cell pairs are read out with a TDC to measure the $\phi$ coordinates. The difference of charge collected at the two ends of the wires allows the measurement of the $z$ coordinate with a precision of $\sim 1.2 \mathrm{~mm}$.

The CMP detector is the second set of muon detectors at CDF, providing a coverage of $|\eta| \leq 0.6$, similar to CMU. The CMP detector is located behind an additional layer of $60 \mathrm{~cm}$ steel, after the CMU. Unlike other parts of the CDF detector, the CMP is not symmetric in $\phi$ coordinate, as can be seen in Fig. 2.10(a), because it is built around the magnet's return yoke. However, since it covers the same $|\eta|$ region as the CMU, the CMP provides enhanced muon identification capabilities. Additionally, the CMP provides a coverage for regions of CMU which are uninstrumented in $\phi$, and also helps to efficiently reject punch-through hadrons, that fake muons in CMU.

The CMX detector extends the muon coverage to $0.6<|\eta|<1.0$. It consists of $15^{\circ}$ conical sections of 12 drift tubes. With the exception of the $30^{\circ}$ slice in $\phi$ in the 
east part of the detector (Fig. 2.10(a)), the east and west parts of CMX are identical. This $30^{\circ}$ region is left empty, to accommodate for the cryogenic electronics for the solenoid.

The IMU detector [51] is the last muon detector, and is used to identify the muons in the $1<|\eta|<1.5$ region, and covers $3 / 4$ of the $\phi$ azimuthal region. The IMU consists of barrel shaped array of muon chambers (BMU) and scintillators (BSU), and is installed on top of the forward toroids (torroids are not powered in Run II). Due to the geometry of installation, the amount of material that particles travel before hitting the IMU varies between around 6 to 20 interaction lengths.

\subsection{Luminosity Counting}

The luminosity at CDF is measured using low pressure gaseous Cherenkov Luminosity Counter (CLC) detector [52], placed at small angles relative to the beam direction, around $3.7<|\eta|<4.7$ at both sides, Fig. 2.11(a). The CLC luminosity monitoring detector is composed of highly segmented array of counters shown schematically in Figure 2.11(b).

The luminosity $\mathcal{L}$ is recorded by the CLC detectors after counting the number of interactions per bunch crossing $\mu$ and using the following equation:

$$
\mu \cdot f_{B C}=\sigma_{i} \cdot \mathcal{L}
$$

where $f_{B C}$ is the frequency of collisions, which for Tevatron is equal to $1.7 \mathrm{MHz}$, and $\sigma_{i}$ is the cross-section of inelastic collisions, which is equal to $\sim 60 \mathrm{mb}$ [53].

\subsection{Trigger and Data Acquisition}

The interaction rate at $\mathrm{CDF}$ is orders of magnitude higher than the rates that the data acquisition system can handle. The bunch crossing rate at the Tevatron is approximately $2.5 \mathrm{MHz}$, since the bunch spacing is $396 \mathrm{~ns}$. However, due to the train structure of the beam, the actual crossing rate is $\sim 1.7 \mathrm{MHz}$. Since it is impossible to 


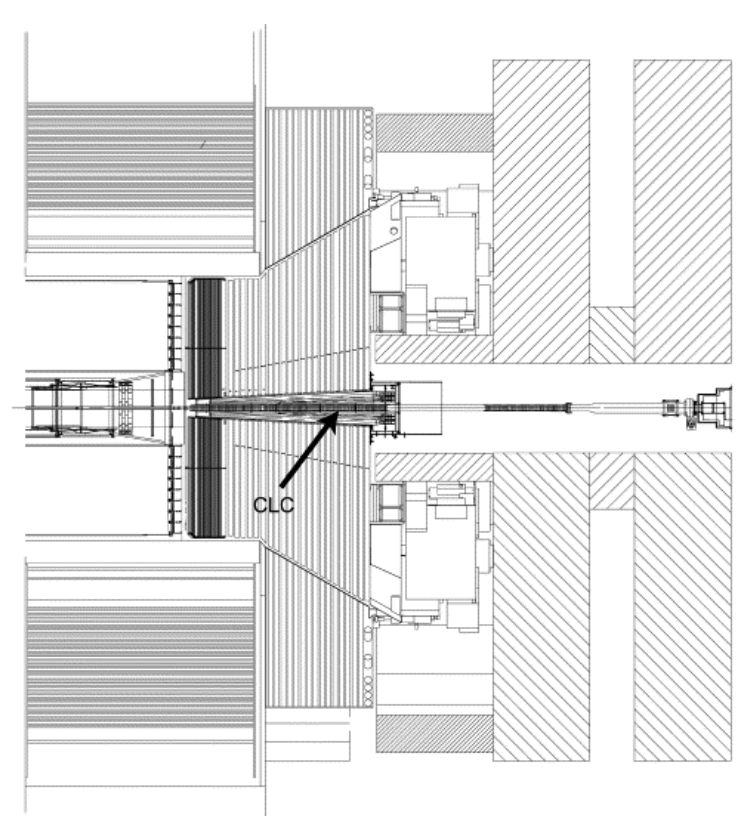

(a)

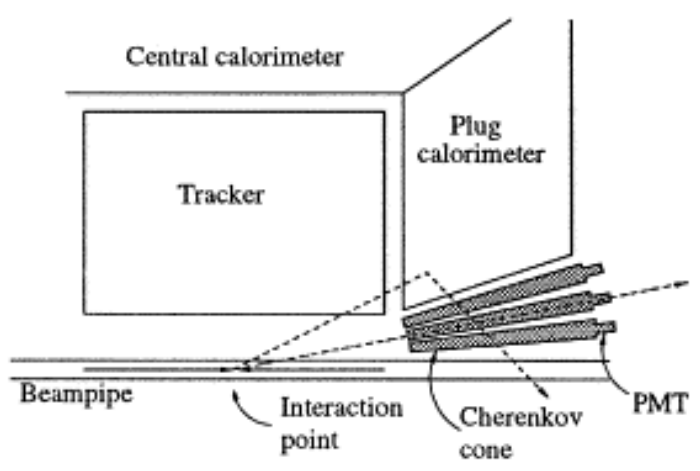

(b)

Figure 2.11.: Cherekov luminosity monitors in CDF: (a) the CLC module shown inside the CDF end-plug calorimeters, (b) schematic view of the luminosity monitor inside a quadrant of CDF.

record each collision, it is necessary to draw decisions whether a specific event is worth to be recorded on an eventbyevent basis. Furthermore, the majority of collisions are not of interest. This leads to an implementation of a system that preselects events online and decides if the corresponding detector data of an event is written to tape or discarded. This is the task of the trigger system, which evaluates if a given event should be read out, reconstructed and stored.

CDF utilizes a three level trigger system [54]. Decisions are made at each level, based on increasingly more complex event information. The functionality of the three level pipelined and buffered trigger system is shown in Fig. 2.12

Level 1 (L1) and Level 2 (L2) are hardware based systems while the Level 3 (L3) filters run on a dedicated computer farm. L1 and L2 hold only a subset of event data, on which they make a decision either to pass it on for subsequent processing or to reject it. 
If an event is accepted at L2 the entire detector is read out and processed in the L3 trigger which consists of a Linux PC farm, where the events are reconstructed and a final Level 3 trigger decision is made. This leads to a further reduction in the output rate by about a factor four. Events that satisfy the L3 trigger requirements are then transfered to the mass storage.

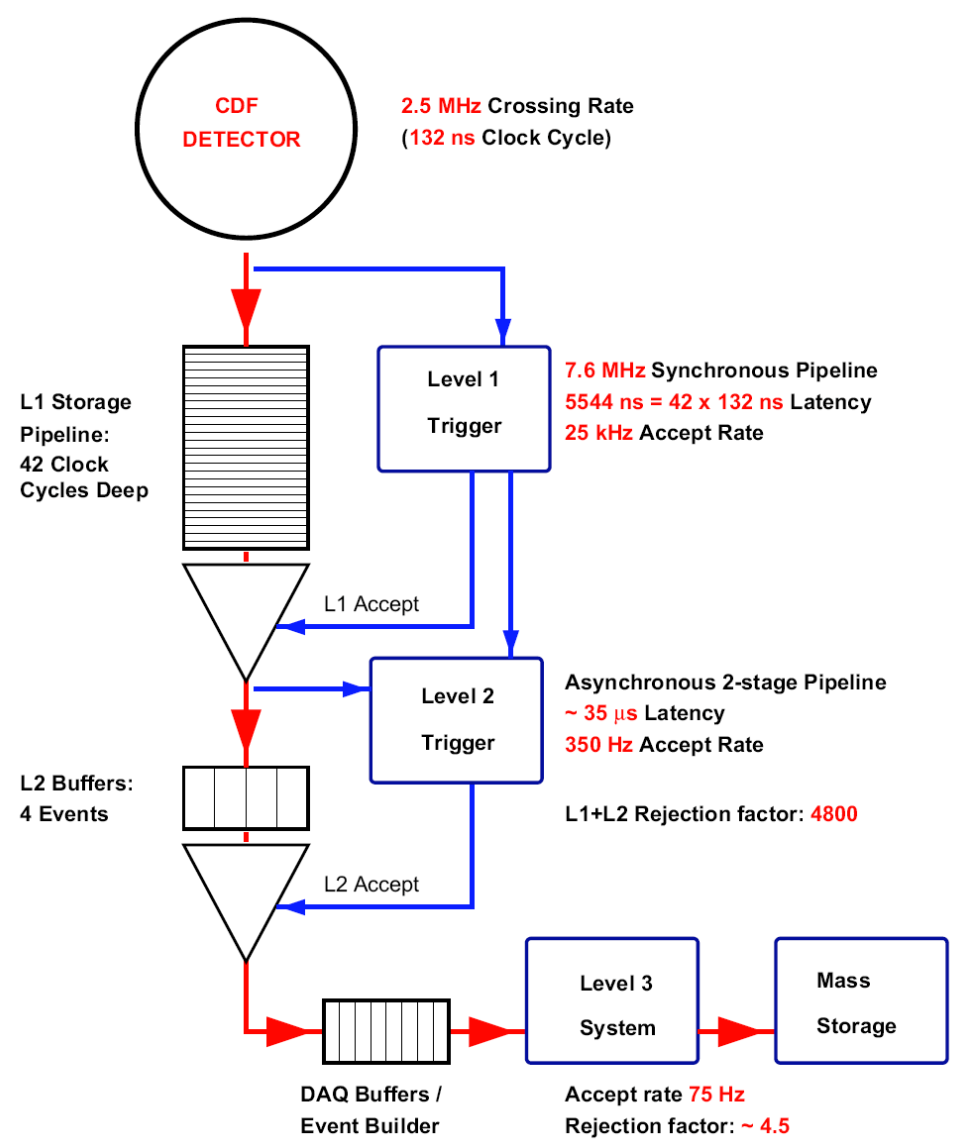

Figure 2.12.: The CDF trigger and data acquisition system. Typical trigger rates and rejection factors for the three-level system are shown. 


\section{RUN II TRIGGER SYSTEM}

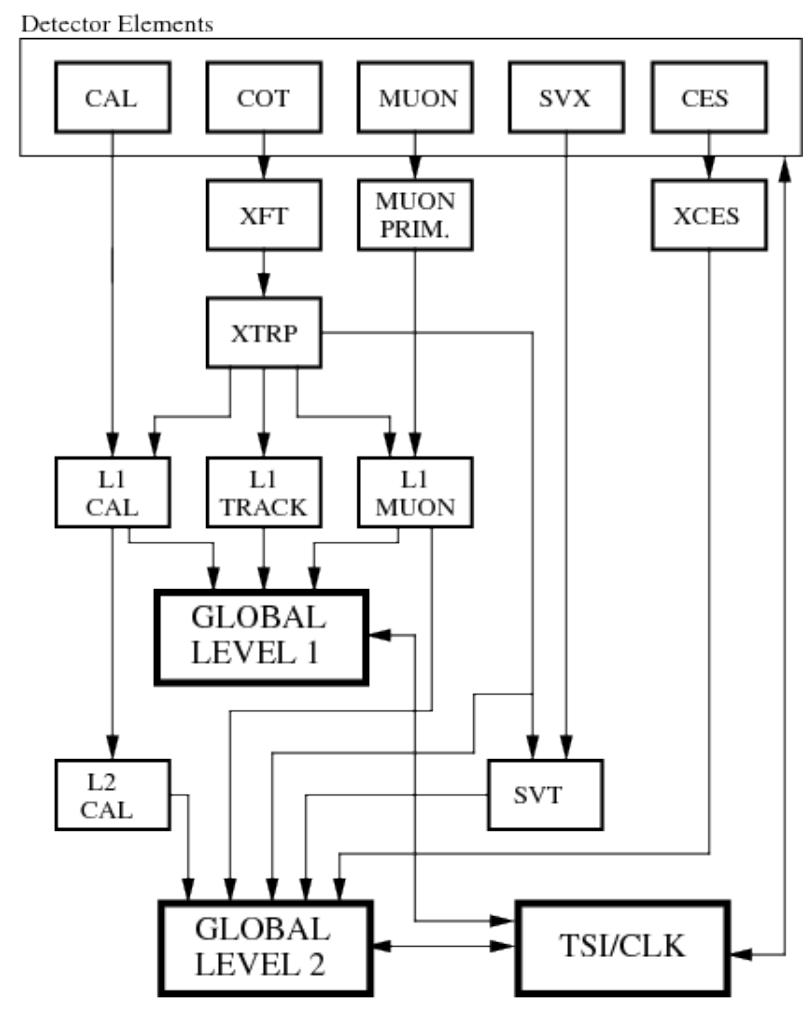

PJW 9/23,96

Figure 2.13.: The Run II trigger-system block diagram.

\subsubsection{Level 1 Trigger}

The L1 trigger finds physics objects based on a subset of the detector. Three parallel systems examine each event: calorimeter trigger boards find calorimeter-based objects, muon trigger cards identify muons and the eXtremely Fast Trigger (XFT) reconstructs tracks in the COT and matches those tracks to energy depositions in calorimeter towers or hits in the muon chambers, Fig. 2.13. Information from all three systems is used independently to determine whether or not to pass the event to the L2 trigger. The decision to keep an event is based on the number and energies of track, electron, photon, muon, $\tau$ lepton and jet candidates, as well as the total 
energy and missing transverse energy in the event. If an event satisfies any of the requirements, it is passed to L2. Several detector systems provide information for the Level-1 trigger decision: the calorimeters, COT, muon systems and TOF. The Trigger Supervisor System (TSI) is responsible for maintaining synchronization and allocating buffer space for each event accepted at Level-1. The typical L1 accept rate is $25 \mathrm{kHz}$.

\subsubsection{Level 2 Trigger}

If an event is accepted by the L1 trigger, all frontend readout components move the data to one of four asynchronous L2 buffers. This buffering is sufficient to allow a $25 \mathrm{kHz}$ L1 accept rate with $\sim 5 \%$ deadtime. At L2 the information from calorimeter, track, and muon based systems, CES and SVX is used to reconstruct physics objects. The L2 cluster finding algorithm combines contiguous regions of calorimeter to form jet candidates. Additionally, CES showermax detectors allow to obtain further positional information on the calorimeter clusters with a better spatial resolution than from calorimeters alone. The Silicon Vertex Trigger (SVT) [55] combines SVX information with the Level 1 tracking, allowing selection of tracks with large impact parameter. Information from the muon system is combined with with tracking system for the muon triggers. The typical L2 accept rate is $350 \mathrm{~Hz}$.

\subsubsection{Level 3 Trigger}

If an event satisfies the L2 trigger, the full detector is read out, and the data from each of the front-end elements is assembled into a single event which is fed to one of the processors in the Level 3 computing farms. In the L3 trigger processor farm, the events are reconstructed and filtered using full event reconstruction, with $\sim 100 \mathrm{~Hz}$ written to permanent storage. 


\title{
Chapter 3. Event Reconstruction
}

\author{
What we observe is not nature itself, but nature \\ exposed to our method of questioning.
}

W. Heisenberg (1901-1976)

\subsection{Event Kinematics}

The dominant production mode for the SM Higgs boson at the Tevatron is through gluon fusion, as shown in Fig.1.6(a). If the Higgs boson mass is below $\sim 135 \mathrm{GeV} / c$, then its dominant decay mode is to two b-quarks, as shown in Fig.1.6(b). Quarks are detected in the calorimeters as showers of particles, or "jets". The searches for a Higgs boson produced via gluon fusion in this mass range have a low sensitivity, due to overwhelming production of QCD multijet events with $b$-quarks. Therefore, the searches mainly focus on the $W / Z$ boson associated production, where the decay products of the vector bosons allow to suppress the backgrounds and provide signatures for triggering.

The three most sensitive signatures for the low mass Higgs searches at the Tevatron are the following:

- events with two identified leptons: $Z H \rightarrow l l b \bar{b}$, cleanest signature, low $\sigma \times B r$,

- events with one identified lepton: $W H \rightarrow l \nu b \bar{b}$, clean signature, high $\sigma \times B r$

- events with no identified leptons: $Z H \rightarrow \nu \nu b \bar{b}$ and $W H \rightarrow \not \nu b \bar{b}$, high $\sigma \times B r$, huge backgrounds from QCD multijet production.

We focus on the latter channel in this analysis. Since the neutrinos from $Z H \rightarrow$ $\nu \nu b \bar{b}$ cannot be detected, their transverse momentum is missing from the total transverse momentum of the event, thus the main characteristic of these events is a large 
imbalance in the transverse energy, called missing transverse energy $\left(\mathbb{E}_{T}\right)$. The same statement holds for the $W H$ events when the lepton produced in the $W$-decay escapes detection. Another important characteristic of the signal is the lack of isolated charged leptons. Hence, the experimental signature for the signal events is: the presence of a large $\mathbb{E}_{T}$ from the decays of the $W / Z$ and at least two jets from the Higgs boson decay.

This section introduces the definitions of the experimental observables used in the Higgs boson search: charged particle tracks, jets, missing transverse energy, $b$-jet identification and charged lepton identification algorithms.

\subsection{Data Format and Analysis Software}

CDF experiment uses the ROOT format [56] for data handling and storage for Run II. This format allows to develop a fully object-oriented data model [57]. The data is stored in a ROOT "ntuple", where the data is organized in data blocks, each block containing a collection of objects of the same type. We use a type of ntuple developed for storage of CDF data called Standard Ntuple ("StNtuple") [58], version 2.4.3. The StNtuples are then processed using ROOT analysis framework, based on $\mathrm{C}++$ programming language.

\subsection{Track Reconstruction}

The tracking system of CDF experiment is introduced in Section 2.2.1. Charged particle trajectories ("tracks") are reconstructed using the tracking system, by combining the measurements ("hits") along the trajectory of a particle.

Inside the CDF tracking system the charged particles move in a uniform magnetic field provided by the solenoid magnet (Section 2.2.4), and follow a helical trajectory. The curvature of the helix depends on the momentum and charge of the particle. At CDF, such helices are described using five parameters, defined with respect to the point of minimum approach to the origin $\left(X_{0}, Y_{0}\right.$ in Figure 3.1$)$ : 


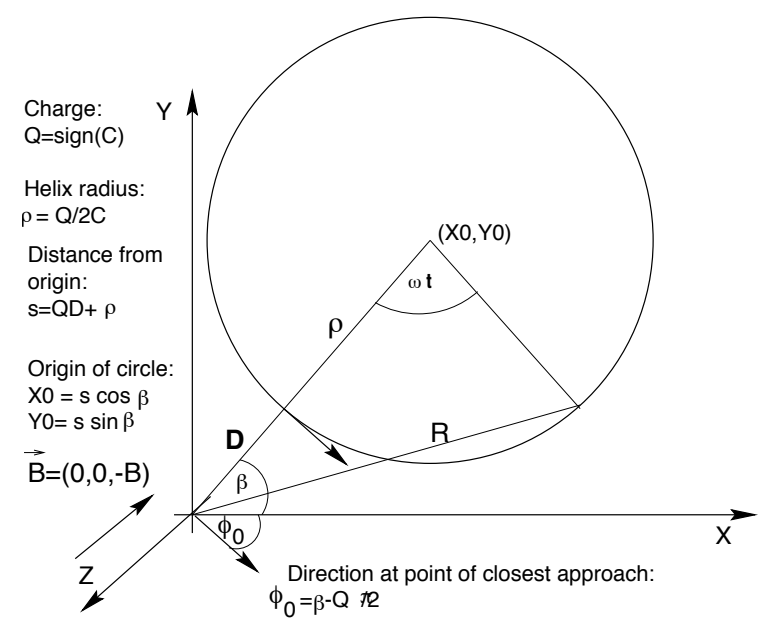

Figure 3.1.: Track of a positively charged particle.

- $\cot \theta$ : cotangent of the polar angle at the trajectory point of closest approach to the origin.

- $C$ : half curvature of the helix circle in the $r-\phi$ plane. $C$ is defined as:

$$
C=\operatorname{sign}(Q) / 2 \rho
$$

where $\rho$ is the radius of the projection of the trajectory to the $r-\phi$ plane.

- $Z_{0}$ : the $z$ position of the trajectory point closest to the origin

- $D$ : signed impact parameter: distance between helix and origin at minimum approach.

$$
D=\operatorname{sign}(Q) \cdot\left(\sqrt{X_{0}^{2}+Y_{0}^{2}}-\rho\right)
$$

- $\phi_{0}$ : direction of track at the point of minimum approach.

The CDF tracking system is composed of two sub-detectors: the inner silicon tracker and the COT (see Section 2.2.1). Track reconstruction [59] starts in the COT, which surrounds the silicon tracker. Because of the larger radius this leads to a lower track density and better separated tracks. The track reconstruction is performed in two steps. First, $r-\phi$ tracks are reconstructed using four axial superlayers. Second, 
for each $r-\phi$ track, the algorithm performs a 3D fit using all combinations of segments in the stereo super-layers that are consistent with the reference track. The newly estimated trajectory is then used to determine which of the segments in the remaining stereo layers are consistent with belonging to the track. The COT tracking efficiency is nearly $100 \%$ for isolated tracks with $p_{T}>5 \mathrm{GeV} / \mathrm{c}$.

The "Outside-In" tracking algorithm extends the COT tracks into the silicon, by extrapolating them into the silicon detectors and attaching silicon hits to the COT tracks. Hits in the silicon tracker that are not associated with COT tracks are used to construct the "Silicon Stand-Alone" tracks. In order to reduce combinatorics, hits that are attached to any other track are not used in this algorithm. The advantage of the Silicon Stand-Alone algorithm is that it allows to extend the tracking coverage up to $|\eta|<2$, while the COT covers only $|\eta|<1$. Another way to reconstruct tracks is

by using the "Inside-Out" algorithm [60], which extrapolates the Silicon Stand-Alone tracks into COT. This algorithm recovers tracks that did not traverse the entire COT volume due to being in the intermediate rapidity region.

\subsection{Primary Vertex Reconstruction}

The location of the hard interaction in the event is referred to as the primary interaction vertex. A precise knowledge of the location of the primary vertex is required to correctly calculate the transverse components of physical observables in the detector, such as transverse energies of jets. Primary vertices are found at the intersection of the prompt tracks that satisfy a certain set of quality requirements. These tracks are iteratively added to the fit to a common origin. If a track causes the $\chi^{2}$ of the fit to exceed a certain threshold, it is removed from the fit ("pruning"). This procedure continues until either the list of tracks is exhausted and a good fit is found, or no vertex can be formed. In cases when several vertices are found, the one with the highest scalar sum of outgoing track $p_{T}$ 's is considered as the primary vertex of the event. The precision for the fitted primary vertex position ranges from 
10-30 $\mu \mathrm{m}$, depending on the number of reconstructed tracks and the topology of the event.

\subsection{Jets}

As mentioned in Section 1.2, quarks and gluons emerging from the hard collision manifest themselves as sprays of particles called "jets". After a quark is created in a collision, the color confinement forces it to pull quark-antiquark pairs from vacuum, to form a color-neutral state. One of the quarks may also radiate a gluon, before forming a bound state. Similarly, a gluon created in the collision or radiated from the quark may split into a quark-antiquark pair and so on. As a result, a shower-like process develops. In the end of this process, color-less hadrons are formed, which then decay to a collimated spray of stable particles that moves towards the detector. Due to their collimated nature, these showers are detected by the calorimeters as energy deposits in localized areas of the detector.

\subsubsection{Jet Reconstruction}

Several algorithms have been developed for reconstructing jets from energy deposits in calorimeter towers. The algorithm used in this analysis is called JETCLU $[61,62]$, which is an iterative fixed cone algorithm. Cone algorithms form jets by associating together towers that lie within a circle of specific radius $\mathrm{R}$ in $\phi-\eta$ space. In this analysis we use jets with a cone radius $R=0.4$ :

$$
R=\sqrt{(\Delta \eta)^{2}+(\Delta \phi)^{2}}<0.4
$$

where $\Delta \eta=\eta_{C}-\eta_{i}$ and $\Delta \phi=\phi_{C}-\phi_{i}$. The variables $\eta_{C}$ and $\phi_{C}$ are the coordinates of the centroid of the jet, which is defined as the $E_{T}$-weighted average of the towers's locations within the jet, where $\eta_{i}$ and $\phi_{i}$ are the locations of the $i^{\text {th }}$ tower. 
The JETCLU jet finding algorithm starts by creating a list of towers with $E_{T}>$ $1 \mathrm{GeV}$, that are sorted by decreasing $E_{T}$ and are used as "seeds" for the jet clustering algorithm. Here, $E_{T}$ is defined as:

$$
E_{T}=E^{E M} \sin \theta^{E M}+E^{H A D} \sin \theta^{H A D}
$$

where $E^{E M} / E^{H A D}$ are the energies measured in the electromagnetic/hadronic section of the tower, $\theta^{E M} / \theta^{H A D}$ are the polar angle with respect to the location of the primary vertex of the event (see Section 3.4). Starting from the seed, a trial centroid is formed by grouping all towers within the cone, starting from the most energetic tower. Once all towers are grouped, the $E_{T}$-weighted centroid is recalculated, including contributions from all towers in the cone, and the new centroid is used for the next iteration. The location and the $E_{T}$ of the centroid changes, until a stable solution is found, which is defined as a JETCLU jet. Overlapping jets are merged if they share more than $50 \%$ of energy.

The quantity $\left(E, p_{x}, p_{y}, p_{z}\right)$ can be computed from the towers associated to the jet. The electromagnetic and hadronic sections of each tower are assigned a massless four-vector with the magnitude equal to the total measured energy, and direction defined by the unit vector pointing from the primary vertex to the location of shower maximum.

$$
\begin{aligned}
& E=\sum_{i=1}^{N_{\text {towers }}}\left(E_{i}^{E M}+E_{i}^{H A D}\right) \\
& p_{x}=\sum_{i=1}^{N_{\text {towers }}}\left(E_{i}^{E M} \sin \theta_{i}^{E M} \cos \phi_{i}^{E M}+E_{i}^{H A D} \sin _{i} \theta^{H A D} \cos \phi_{i}^{H A D}\right) \\
& p_{y}=\sum_{i=1}^{N_{\text {towers }}}\left(E_{i}^{E M} \sin \theta_{i}^{E M} \sin \phi_{i}^{E M}+E_{i}^{H A D} \sin _{i} \theta^{H A D} \sin \phi_{i}^{H A D}\right) \\
& p_{z}=\sum_{i=1}^{N_{\text {towers }}}\left(E_{i}^{E M} \cos \theta_{i}^{E M}+E_{i}^{H A D} \cos _{i} \theta^{H A D}\right)
\end{aligned}
$$


The direction and the transverse energy of a jet is then determined by:

$$
\begin{aligned}
& \theta=\arctan \left(\frac{\sqrt{p_{x}^{2}+p_{y}^{2}}}{p_{z}}\right) \\
& \phi=\arctan \left(\frac{p_{y}}{p_{x}}\right) \\
& E_{T}=E \sin \theta
\end{aligned}
$$

\subsubsection{Generic Jet Energy Corrections}

The energies of the jets measured by the CDF calorimeter ( "raw" energies) need to be corrected to account for various instrumental effects, such as uninstrumented regions of the detector, non-homogeneous response over various $\eta$ regions, and to account for the energy coming from pile-up events ${ }^{1}$. Additionally, the calorimeter response needs to be corrected in order to be able to associate the measured energy to the parent parton energy, so that direct comparisons can be made with other experiments and theoretical predictions [62]. The original parton transverse energy is then estimated by:

$$
p_{T}^{\text {parton }}=\left(p_{T}^{j e t} \times C_{\eta}-C_{M I}\right) \times C_{A b s}-C_{U E}+C_{O O C}
$$

where the $p_{T}^{\text {parton }}$ is the transverse momentum of the parent parton, $p_{T}^{\text {jet }}$ is the transverse momentum measured in the calorimeter jet and the various correction factors are:

- $C_{\eta}$ : this correction is applied to raw jet energies to ensure a homogeneous response of the calorimeter over the entire $\eta$ range. The $\eta$ dependence of the calorimeter response arises from the presence of uninstrumented regions: a uninstrumented region ( "crack") around $\eta=0$ (see Section 2.2.5) where the west and east parts of the calorimeter are joined, and cracks at $|\eta|=1.1$ where the central and plug calorimeters are joined. Additionally, the difference in response of the central and plug calorimeters causes $\eta$ dependence. The $C_{\eta}$ corrections

\footnotetext{
${ }^{1}$ Energy coming from a different collision in the same bunch-crossing
} 
also correct for the transverse spreading of calorimeter showers outside the jet cone and any $\eta$ dependence of gluon radiation and multiple parton interactions.

- $C_{M I}$ : at high instantaneous luminosities multiple $p \bar{p}$ collisions may occur during the same bunch crossing. These additional interactions result in an apparent increase of the jet energies, if the hadrons arising from them accidentally overlap with the jets from the primary interactions. The $C_{M I}$ corrections subtract that extra energy.

- $C_{A b s}$ : this correction ( "Absolute") aims to transform the jet energy measured in the calorimeter into the momentum of the particle jet. Energies of the calorimeter jets after this correction are independent of the CDF detector, and can be compared to other experiments or theory. This corrections are derived from a detailed simulation of the physics processes and the detector response. The calorimeter simulation was optimized to reproduce the measured single particle response, therefore corrections over a large range of jet energies can be derived.

- $C_{U E}$ and $C_{O O C}$ : this corrections are applied to calorimeter jets to account for parton radiation and hadronization effects due to the finite size of the jet cone algorithm. Corrections for the Underlying Event (UE) account for the energy deposited in the jet cone originating not from the actual parton of the hard interaction, but from particles from the initial state gluon radiation, or hadrons from the break-up of the colliding proton or antiproton. The Out-Of-Cone (OOC) corrections account for the fact that a fraction of the energy of the parent parton can be lost from the jet cone due to low $p_{T}$ particles bending in the magnetic field or gluon radiation at large angles with respect to the parent parton.

The total systematic uncertainty on the jet energy are shown in Figure.3.2

In this analysis we use jet energy corrections corresponding to $C_{\eta}, C_{M I}$ and $C_{A b s}$, which is common for MET+Jets analysis at CDF. Additionally, we correct jet energies using the tracking system, employing an algorithm described in the next section. 


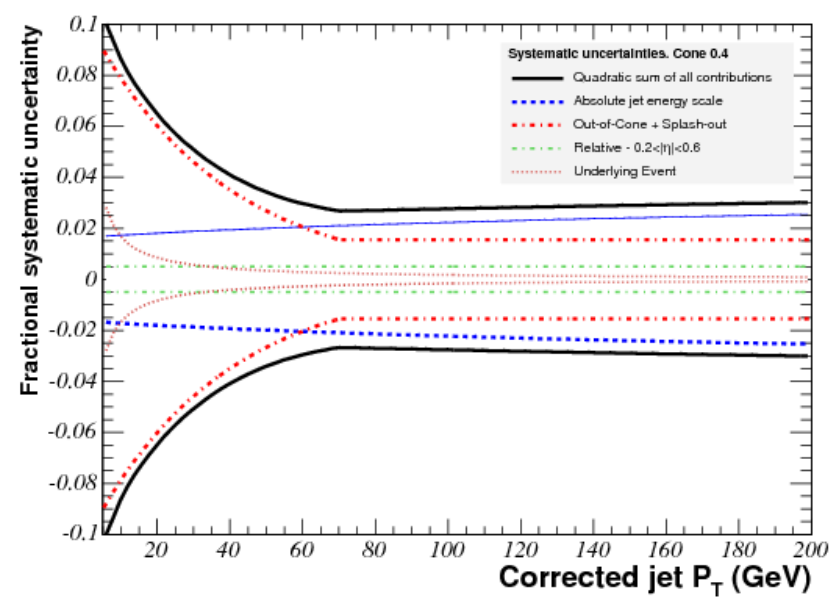

Figure 3.2.: Total systematic uncertainty on the jet energy scale.

\subsubsection{Jet Energy Corrections using H1 algorithm}

The vast majority of analyses at CDF employ the generic jet energy corrections described in the previous section, that rely only on calorimeter information. Several experiments have successfully improved the calorimeter jet reconstruction, by combining the measurement of the momentum of charged particles in the tracking detectors with the information provided by calorimeter energy deposits $[63,64]$.

Hadronic jets are mainly composed of $\pi^{ \pm}, \pi^{0}, K^{ \pm}, K_{S}, K_{L}$, protons and neutrons. Due to the fact that the majority of the particles in a jet have low momenta, the tracking detectors are able to measure their momenta to a much higher precision. Decays of a $\pi^{0}$ to a pair of photons are reconstructed in the EM calorimeter. $K_{S}$ usually decay to $\pi^{+} \pi^{-}$or $\pi^{0} \pi^{0}$, hence can be reconstructed either in the tracking or calorimeter systems. Only $K_{L}$ and the neutrons must usually be reconstructed by the hadronic calorimeter. Therefore, in principle it is possible to reconstruct a large portion of the jet energy using the tracking system for hadronic particles, and the EM calorimeter for the electromagnetic particles. This has the advantage of a improving the estimate of the jets' true energy, since the energy resolution of the hadronic calorimeter is much worse than in the electromagnetic calorimeter (see 
Table 2.2). However, the spatial extent of hadronic showers is not sufficiently small to isolate hadronic from electromagnetic showers, leading to modest improvements in jet energy resolution.

In this analysis we utilize an algorithm based on the method developed and successfully used by the H1 collaboration [65]. The correction to the jet energy obtained from this method is applied in addition to the generic corrections described in Section 3.5 .2 .

Tracks used in this algorithm must satisfy the following requirements, in order to have a reliable momentum measurement:

- $p_{T}^{\text {track }}>0.5 \mathrm{GeV} / c$

- $p_{T}^{\text {track }}<15.0 \mathrm{GeV} / c$

- $N_{\mathrm{COT}}>25$ for $\left|\eta_{\text {track }}\right|<0.8$

- $Z_{0}<60 \mathrm{~cm}$

where $p_{T}^{\text {track }}$ is the transverse momentum of the track, $N_{C O T}$ is the number of hits in the COT detector associated to the track, $\eta_{\text {track }}$ is the pseudo-rapidity of the track and $Z_{0}$ is the distance of the closest approach to the beam line. The list of tracks satisfying the above conditions is then sorted on the basis of ascending $p_{T}^{\text {track }}$ and extrapolated to the surface of the calorimeter. All towers with $\Delta \eta<0.1$ and $\Delta \phi<0.2$ are considered as possibly containing energy from the track, based on simulation of single pion events.

The selected towers are sorted in order of distance to the track. If the track energy $^{2}$ is greater than the total energy of the selected towers, the energy in the towers is replaced with the track energy and the towers are removed from further consideration ("locked"). If this is not true, the energy in the first $n$ towers:

\footnotetext{
${ }^{2}$ Assuming that the particle leaving the track is a pion
} 


$$
\begin{aligned}
& \sum_{i=1}^{n} E_{i}^{\text {tower }} \leq E^{\text {track }} \\
& \sum_{i=1}^{n+1} E_{i}^{\text {tower }}>E^{\text {track }}
\end{aligned}
$$

are locked, where $E_{i}^{\text {tower }}$ is the energy measured by the $i^{t h}$ tower. The energy of the $(n+1)^{t h}$ tower is scaled such that the total locked energy is equal to the track energy. The process is repeated for each track. Energy already locked by a previous track is not considered for subsequent tracks. The total energy is then the sum of all quality selected tracks and all non-locked tower energies.

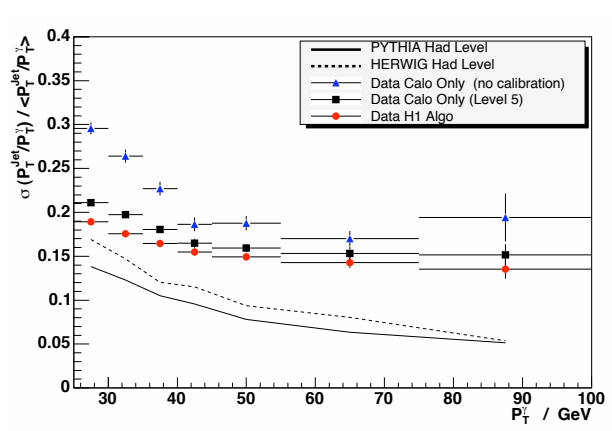

(a)

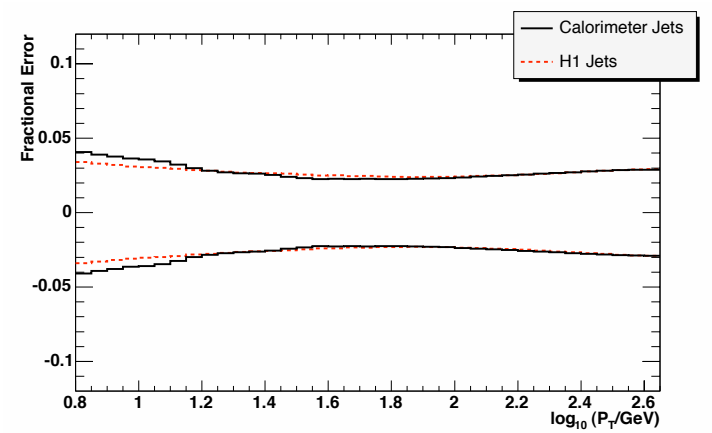

(b)

Figure 3.3.: Performance of the H1 corrections compared to the generic jet energy corrections [65]: (a) comparison of the jet energy resolution for jets in $0.1<|\eta|<0.7$ after generic and $\mathrm{H} 1$ corrections, (b) comparison of the average uncertainty on the jet energy scale.

In events with jets there may be many particles falling into a single tower, and there could be neutrals alongside each charged track. This would result in the calorimeter energy exceeding the track energy. The H1 algorithm will then return the calorimeter energy for these high density regions, resulting in no improvement in the measurement. Usually, events with jets contain regions of both high and low density. Therefore, the H1 algorithm provides modest improvements in resolution, 
$\sim 10 \%$, compared to calorimeter only jets, as shown in Figure 3.3. The results would be more substantial for a detector with a finer granulated calorimeter, and in fact, the calorimeters for the next linear collider are designed to be able to separate individual particles. However, even small improvements in energy resolution are crucial for Higgs boson searches in low mass where $H \rightarrow b \bar{b}$, as reported in [66], substantially improves the sensitivity of the searches. Additionally, the $\mathrm{H} 1$ algorithm helps to increase the acceptance to signal events by $\sim 10 \%$ in this analysis. Energetic jets may sometime appear as low $p_{T}$ jets, since part of the energy is lost in, e.g. uninstrumented regions of the calorimeter. $\mathrm{H} 1$ algorithm helps to recover this kind of events by properly reconstructing their energy, allowing to keep the event that would have otherwise been discarded by the analysis event selection (Section 4.2).

\subsection{Missing Transverse Energy: $\not_{T}$}

Protons and anti-protons that collide at the Tevatron have equal and opposite momenta. Therefore, the total vector momentum sum in an event should be zero. The hard collision happens between the partons of the proton and antiproton, and they can carry any fraction of the parent proton or antiproton. However, since the partons usually have very little momentum in the plane transverse to the beam, the transverse momentum can be considered as a conserved quantity to a good approximation. Any transverse momentum imbalance in the detector may indicate that a weakly interacting particle (e.g. a neutrino $\nu$ ) left the detector without interacting with its material. One of the signal production channels studied in this analysis is $Z H \rightarrow \nu \nu b \bar{b}$, which contains neutrinos that escape detection. Additionally, if the charged lepton $\ell$ from $W H \rightarrow \ell \nu b \bar{b}$ is not detected, the event will also appear to have a large apparent momentum imbalance. Therefore, the transverse momentum imbalance: $\mathbb{\phi}_{T}$, is one of most important quantities in this analysis.

The $x$ and $y$ components of the raw missing transverse energy of the event are obtained from: 


$$
\begin{aligned}
& \mathscr{E}_{x}=-\sum_{i=1}^{N_{\text {towers }}} E_{T}^{i} \cos \phi_{i} \\
& \mathscr{E}_{y}=-\sum_{i=1}^{N_{\text {towers }}} E_{T}^{i} \sin \phi_{i}
\end{aligned}
$$

where the sum is taken over all towers that are above a threshold of $0.1 \mathrm{GeV}$, and the total electromagnetic and hadronic energy in $i^{\text {th }}$ tower is $E_{T}^{i}$. The magnitude of the missing energy is then calculated by:

$$
\mathscr{E}_{T}=\sqrt{\mathscr{E}_{x}^{2}+\mathbb{E}_{y}^{2}}
$$

The azimuthal direction of the $\mathbb{E}_{T}$ is then given by:

$$
\phi_{\not_{T}}=\tan ^{-1}\left(\frac{\mathscr{E}_{y}}{\mathbb{E}_{x}}\right)
$$

While a large $\mathbb{E}_{T}$ is recorded in events that contain an escaping neutrino, other types of processes may also lead to the experimental signature of $\notin_{T}$ :

- when protons or antiprotons of the Tevatron beam collide with nuclei of gas atoms or beam collimators, they produce a "halo" of muons, travelling roughly parallel to the beam. Some of these muons cross a row of calorimeter towers along the z-axis depositing energy to the calorimeters asymmetrically in $\phi$.

- problems with some of the calorimeter tower electronics/calibrations may cause the tower to report a wrong value of the energy of the incident particles.

- cosmic muons traveling through the detector, promoting low $\mathbb{E}_{T}$ to a higher value.

- muons carrying a large momentum can be created in the hard collision. Being minimum ionizing particles, these muons can pass through the calorimeter, without substantial energy loss.

- proton or antiproton beam remnants and beam losses in very forward regions. 
- when the direction of an energetic jet is near an uninstrumented calorimeter region, the energy of the jet will be underestimated. A configuration of two jets that are produced back-to-back with the same momentum will appear to have a momentum imbalance.

\subsection{1 $\not_{T}$ Corrections}

The $\notin_{T}$ measured by the CDF calorimeter ( $r a w " \notin_{T}$ ) needs to be corrected for the same reasons that the jet energies do, as described in Section 3.5.2. Hence, the $\mathbb{E}_{T}$ needs to be recomputed using the corrected values of the jet energies. The event $\mathscr{E}_{T}$ is corrected using the corrected jet $E_{T}^{C o r r}$ values with the following formula:

$$
\begin{aligned}
& \mathscr{E}_{x}^{\text {Corr }}=\not_{x}^{\text {Raw }}-\sum_{i=1}^{N_{\text {jets }}}\left(E_{x}^{\text {Corr }, i}-E_{x}^{\text {Raw }, i}\right) \\
& \mathbb{E}_{y}{ }^{\text {Corr }}=\mathbb{E}_{y}{ }^{\text {Raw }}-\sum_{i=1}^{N_{j e t s}}\left(E_{y}^{\text {Corr }, i}-E_{y}^{\text {Raw }, i}\right)
\end{aligned}
$$

where the $\not_{x}{ }^{R a w}$ and $\notin_{y}{ }^{\text {Raw }}$ are obtained using Equations 3.13-3.14. The azimuthal direction of the corrected $\notin_{T}$ is recomputed using:

$$
\phi_{\not_{T} \text { Corr }}=\tan ^{-1}\left(\frac{\notin_{y}^{C o r r}}{\notin_{x}^{C o r r}}\right)
$$

The $\not_{T}$ energy used everywhere in this analysis is the corrected $\mathbb{E}_{T}$.

\subsubsection{Missing Transverse Momentum: $\not P_{T}^{t r}$}

Most of the physics processes considered in this analysis do produce real high $\not_{T}$ originating from neutrinos or muons, which escape the detection in calorimeter. Additionally, $b$-quarks produced in an event can decay semi-leptonically, which also produce real $\notin_{T}$. Mis-measurements in calorimeter, on the other hand, can cause a QCD dijet event which has no real $\not_{T}$ to appear as if there is energy imbalance in transverse plain. Since QCD multijet production has a very large cross-section, these 
events constitute a big fraction of the analysis data sample. As a way to get a better estimate of the event true missing energy we calculate the $\not_{T}^{t r}[67]$, which is defined as magnitude of the negative vector sum of transverse momenta of charged particles, measured in the tracking system of CDF detector:

$$
\not_{T}^{\text {tr }}=-\left|\sum_{\text {tracks }} p_{T}^{i} \hat{n}_{i}\right|
$$

where $\hat{n_{i}}$ is a unit vector, pointing along the $i^{\text {th }}$ track direction. For the events with true missing energy, $\not_{T}^{t r}$ is highly correlated with calorimeter $\not_{T}$, while for events with mismeasured jets it is not [68]. Thus, $\not_{T}^{t r}$ provides an additional handle to separate mismeasurements from real $\mathbb{E}_{T}$ events. Additionally, the advantage of adding the tracking information lies in the different correlation of the $\not_{T}$ and $\not_{T}^{t r}$ with detector effects.

For the track quality criteria for $\not_{T}^{\text {tr }}$ calculation we used the same selections as those used in the calculation of the position of the event primary vertex in CDF. The selections are listed in Table 3.1 and only tracks satisfying these conditions are used when calculating $\not_{T}^{t r}$. The quantities used in Table 3.1 are:

- $N_{C O T}$ : the total number of hits in the COT detector

- $N_{S V X}$ : the total number of hits in the SVX detector

- $N_{C O T}^{A x}(\geq 5$ hits $)$ and $N_{C O T}^{S t}(\geq 5$ hits $)$ : the number of axial and stereo COT layers that have at least 5 hits.

- $N_{S V X}^{A x}$ and $N_{S V X}^{S t}$ : the number of hits in axial and stereo layers in SVX

- $\chi^{2}$ : the $\chi^{2}$ value of the track fit

- $\chi_{S V X}^{2}$ : the $\chi^{2}$ value of the track fit, using only information from SVX detector

Additionally we only use tracks with $0.5 \mathrm{GeV}<p_{T}<200 \mathrm{GeV},|\eta|<1.5$ and matching primary vertex $Z$ position within $|\Delta Z|<2.0 \mathrm{~cm}$. 
Table 3.1: Quality requirements for tracks used in $\not P_{T}^{t r}$ calculation. The rows are listed in the order the selections are checked. If the first row requirements fail, we next check the second row, etc.

\begin{tabular}{|c|c|}
\hline Category & Quality requirements \\
\hline \hline Strong COT part & $N_{C O T}>0$ \\
& $\left(\chi^{2}-\chi_{S V X}^{2}\right) /\left(N_{C O T}-5\right)<4.0$ \\
& $N_{C O T}^{S t}(\geq 5$ hits $) \geq 3, N_{C O T}^{A x}(\geq 5$ hits $) \geq 3$ \\
\hline COT part is weak, good $\chi^{2}$ & $N_{C O T}>0$ \\
& $\left(\chi^{2}-\chi_{S V X}^{2}\right) /\left(N_{C O T}-5\right)<4.0$ \\
& $N_{C O T}^{S t}(\geq 5$ hits $) \geq 2, N_{C O T}^{A x}(\geq 5$ hits $) \geq 2$ \\
COT part really weak, good $\chi^{2}$ & $N_{S V X}^{A x} \geq 4, N_{S V X}^{S t} \geq 3$ \\
& $\chi_{S V X}^{2} /\left(N_{S V X}-5\right)<8.0$ \\
\hline Inside-Out or silicone stand-alone & $N_{C O T}>0$ \\
& $\left(\chi^{2}-\chi_{S V X}^{2}\right) /\left(N_{C O T}-5\right)<4.0$ \\
& $N_{S V X}^{A x} \geq 5, N_{S V X}^{S t} \geq 3$ \\
& $\chi_{S V X}^{2} /\left(N_{S V X}-5\right)<8.0$ \\
\hline & $N_{S V X}^{A x} \geq 5, N_{S V X}^{S t} \geq 3$ \\
& $\chi_{S V X}^{2} /\left(N_{S V X}-5\right)<8.0$ \\
\hline
\end{tabular}

If the track satisfies the selection criteria, we add it to the event $\not_{T}^{t r}$ calculation. The direction of $\not P_{T}^{t r}$ is calculated similar to $\not_{T}$

$$
\varphi_{\not p_{T}^{t r}}=\arctan \left(\frac{\not p_{y}^{t r}}{\not P_{x}^{t r}}\right)
$$

where $\not P_{x}^{t r}$ and $\not y_{y}^{t r}$ are sums of track momenta in $x$ and $y$ directions respectively. 


\subsection{Identification of $b$-quark Jets: $b$-tagging}

The identification of jets originating from $b$-quarks plays a crucial role in searches for a Higgs boson with a mass below $135 \mathrm{GeV} / c^{2}$, since in this mass range Higgs boson decays predominantly into a $b \bar{b}$ pair. The $b$-quark hadronizes almost immediately, forming a $b$ meson or baryon, such as $B^{0}(d \bar{b})$ or $\Lambda_{B}(u d b)$. Since the $b$-quark decays through a weak force, its life-time is long enough to move a considerable distance before decaying to lighter hadrons, typically travelling a few millimeters away from the primary vertex. Reconstruction of the decay products of the $b$-hadron allows to look for the trajectories of the decay products that have a large impact parameter. Different algorithms exist at CDF to identify jets that originate from hadronization of $b$-quarks, two of them are used in this analysis:

- Secondary Vertex Tagging algorithm (SecVtx) [69] reconstructs a secondary displaced vertex in the jet. Several of the displaced tracks from the decay of the $b$ hadron can be determined to originate from a common location and can be used to construct a displaced, "secondary", vertex.

- Jet Probability algorithm (JetProb) [70] takes advantage of the lifetime of $b$ hadrons. This algorithm uses tracks associated with a jet to determine the probability for these to come from the primary vertex of the interaction.

The SecVtx algorithm operates on a per-jet basis, taking into consideration only tracks within the jet cone. Tracks are required to satisfy a set of selections based on their transverse momentum, the number of silicon hits attached to the tracks, the quality of those hits, and the $\chi^{2}$ of the final track fit. Jets that have at least two tracks passing these selections are defined as "taggable". Displaced tracks in the jet are selected based on the significance of their impact parameter with respect to the primary vertex. Tracks satisfying the quality requirements are used to form the secondary vertex. To reduce the contribution from the false secondary vertices, a good secondary vertex is required to have $L_{2 D} / \sigma_{L_{2} D}>3$, where $L_{2 D}$ is the displacement of the secondary vertex with respect to the primary vertex in the transverse plane, and 
$\sigma_{L_{2} D}$ is total estimated uncertainty on $L_{2 D}$ including the error on the primary vertex position. The sign of $L_{2 D}$ indicates the position of the secondary vertex with respect to the primary vertex. If the angle between the jet axis and the vector pointing from the primary vertex to the secondary vertex is less than $\pi / 2, L_{2 D}$ is positive; otherwise, it is negative. If $L_{2 D}$ is positive, the secondary vertex points towards the jet direction, as in true b hadron decays. For negative $L_{2 D}$ the secondary vertex points away from the jet, which may happen as a result of mismeasured tracks.

The JetProb algorithm is used to determine whether a jet has been produced from the hadronization process of a light parton ( $u, d, s$ quark or a gluon) or a heavy parton: $b$ or $c$ quark. The calculation of the probability is based on the impact parameters of the tracks in the jet and their uncertainties. Tracks are required to satisfy a set of selections based on to the number and quality of COT and SVX hits, detector $\eta, p_{T}$ of the tracks and the impact parameter significance. The probabilities of multiple tracks are combined to from an overall probability, which is interpreted as the likelihood that a jet does not contain secondary vertices from a long-lived hadron. The probability for tracks originating from the primary vertex is uniformly distributed from 0 to 1 . For a jet coming from $b$-quark hadronization, the distribution peaks at 0 , due to tracks from long-lived particles that have a large impact parameter with respect to the primary vertex.

CDF uses three operating points for SecVtx algorithm, with an increasing purity of the selected $b$-jets at the cost of decreased efficiency: Loose, Tight and UltraTight operating points. In this analysis, we use the Tight setting. The efficiencies of these operating points are shown in Figure 3.4. The efficiency to identify a jet originating from a $b$-quark with SecVtx was measured in CDF data and in the simulation, and was observed to be higher in simulation. Hence, a scale factor is applied to the simulated events to make the efficiencies match. The scale factor was measured to be $\epsilon_{M C}=0.95 \pm 0.04$.

Since the JetProb provides a continuous output, any operating point can be selected to be used, to optimize the signal-to-background point for a specific analysis. 


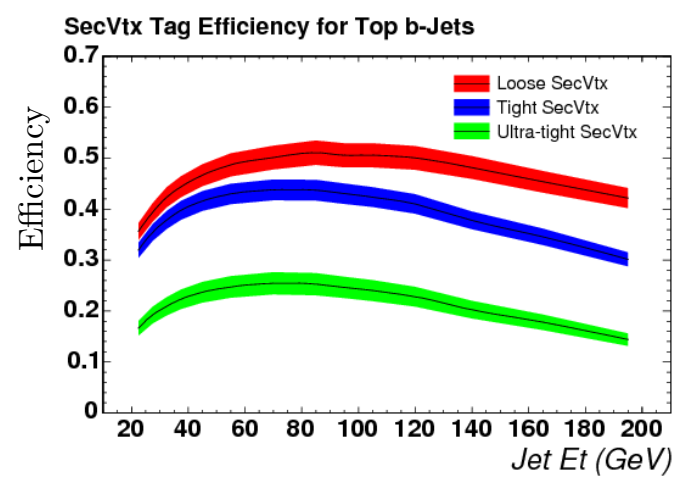

(a)

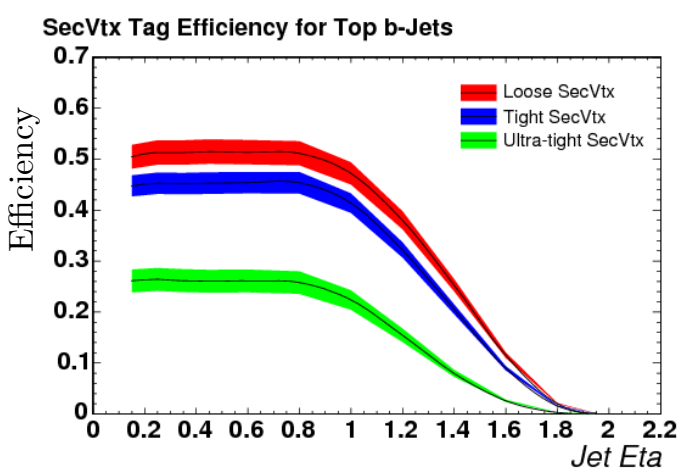

(b)

Figure 3.4.: Tagging efficiencies for the SecVtx algorithm as a function of jet (a) $E_{T}$ and (b) $|\eta|$.

We use the operating point corresponding to the "loose" convention used in CDF, where the jet is considered as $b$-tagged if the JetProb value $P_{J}$ is below $5 \%$. The efficiency of these operating point as a function of jet $E_{T}$ is shown in Figure 3.5. The scale factor to account for simulation/data difference for JetProb algorithm at $P_{J}<5 \%$ operating point was measured to be $\epsilon_{M C}=0.85 \pm 0.07$.

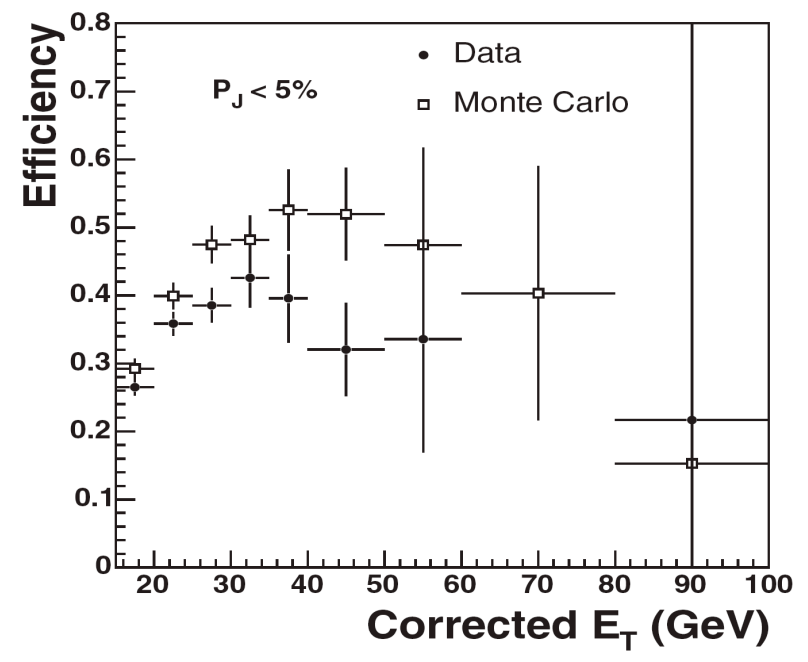

Figure 3.5.: Tagging efficiencies for the JetProb algorithm as a function of jet $E_{T}$. 


\subsection{Lepton Identification}

One of the distinctive features of this analysis is the absence of any identified charged leptons in the final state, due to $Z \rightarrow \nu \nu$ decay, or $W \rightarrow \ell \nu$ where the lepton is not detected. Hence, our goal is to reject all events that contain an identified electron $e^{ \pm}$or muon $\mu^{ \pm}$. We use loose lepton identification selections in order to ensure high efficiency of lepton rejection, as well as to keep the data sample used in this analysis statistically independent from dedicated searches with identified leptons.

\subsubsection{Electron Reconstruction}

Electrons are identified by the electromagnetic calorimeter (see Section 2.2.5) CEM and PEM. The identification selections are different for CEM and PEM, and are listed in Table 3.2. Electron candidates identified in the central calorimeter must also match a track in the COT. The quantities used to identify electron candidates are:

- total transverse energy of the electron cluster $E_{T}$ in the electromagnetic calorimeter

- the ratio between the cluster energy to the momentum of the associated track $E / P$

- the ratio of the total hadronic cluster energy to the total EM energy $E^{H A D} / E^{E M}$. Electrons are expected to deposit most of their energy in the electromagnetic part of the calorimeter.

- the lateral shower profile of the electromagnetic shower $L_{s h r}$, which is a measure of lateral sharing between adjacent towers in the calorimeter. Most electrons deposit their energy in a single tower, since the typical lateral size of the electromagnetic shower is smaller than the size of a tower. 
Table 3.2: Electron identification selections using central and plug calorimeters.

\begin{tabular}{|c|c|}
\hline \multicolumn{2}{|c|}{ Central Electrons } \\
\hline \hline$E_{T}$ & $\geq 10 \mathrm{GeV}$ \\
$E / P$ & $<4$ \\
$E^{H A D} / E^{E M}$ & $<0.125$ \\
$L_{\text {shr }}$ & $<0.2$ \\
$|\Delta X|$ & $<3 \mathrm{~cm}$ \\
$|\Delta Z|$ & $<5 \mathrm{~cm}$ \\
$I s o 4$ & $<0.2$ \\
$\chi^{2}$ & $<10$ \\
\hline \hline \multicolumn{2}{|c|}{ Plug Electrons } \\
\hline$E_{T}$ & $\geq 10 \mathrm{GeV}$ \\
$E^{H A D} / E^{E M}$ & $<0.125$ \\
$\chi^{2}(3 \times 3)$ & $<10$ \\
\hline
\end{tabular}

- $\Delta X$ and $\Delta Z$, the distances in the transverse plane and in the $z$-direction between the cluster position from the showermax measurements and the extrapolated track.

- Iso4, referred to as the "track isolation", defined as a ratio of the sum of $p_{T}$ of all tracks in a cone radius $\Delta R=\sqrt{(\Delta \eta)^{2}+(\Delta \phi)^{2}}<0.4$ around the electron track candidate and the $p_{T}$ of the electron candidate.

- $\chi^{2}$, the result of the comparison of the CES shower profile of the electron candidate with the measurements with the test beam electrons.

- $\chi^{2}(3 \times 3)$, the result of the comparison of the PEM shower profile of the electron candidate with the measurements with the test beam electrons. The test beam results for PEM were obtained using $3 \times 3$ cluster size.

Any event that have an electron candidate passing the identification requirements listed in Table 3.2 is discarded from this analysis. 


\subsubsection{Isolated Tracks}

Muons are identified using the muon detectors at CDF (see Section 2.2.6). Since muon reconstruction at $\mathrm{CDF}$ requires to associate muon stubs to the extrapolated COT track, all events containing an isolated track are discarded from this analysis, based on the selection criteria listed in Table 3.3. These selection provides a very high efficiency of rejecting all potential muon candidates. The quantities used to identify muon candidates are:

- $p_{T}$, transverse momentum of the isolated track

- $E^{E M}$ and $E^{H A D}$, the energy deposits in the electromagnetic and hadronic parts of the calorimeter associated with the track.

- $Z_{0}$, the $z$-coordinate of the track at the point of closest approach to the detector origin,

- $I s o 4 / p_{T}$, relative track isolation, where $I s o 4$ is define in previous section

- $N_{C O T}^{A x}$ and $N_{C O T}^{S t}$, number of axial and stereo COT layers with at least 5 hits.

Table 3.3: Summary of isolated track identification selections.

\begin{tabular}{|c|c|}
\hline \multicolumn{2}{|c|}{ Isolated Tracks } \\
\hline$P_{T}$ & $\geq 10 \mathrm{GeV}$ \\
$E^{E M}$ & $>0$ and $<2 \mathrm{GeV}$ \\
$E^{H A D}$ & $>0$ and $<6 \mathrm{GeV}$ \\
$\left|Z_{0}\right|$ & $<60 \mathrm{~cm}$ \\
$I s o 4 / p_{T}$ & $<0.1$ \\
$N_{C O T}^{A x}(\geq 5)$ & $\geq 3$ \\
$N_{C O T}^{S t}(\geq 5)$ & $\geq 2$ \\
\hline
\end{tabular}




\section{Chapter 4. Trigger Path and Event Preselection}

To write it, it took three months; to conceive it three minutes; to collect the data in it - all my life.

F. Scott Fitzgerald (1896 - 1940)

One of the main challenges at hadron colliders, such as the Tevatron, is the design of an efficient data acquisition system. While, the collision rate at the Tevatron is about $1.7 \mathrm{MHz}$, the vast majority of collisions do not proceed with a large momentum transfer. As it was mentioned in Section 2.3, the cross-section of inelastic collision is about $60 \mathrm{mb}$. The processes of interest usually have smaller cross sections. For example, top quark pair production has a cross section of $\sim 7 \mathrm{pb}$, which is a factor of $10^{10}$ smaller than that of inelastic collisions! Additionally, the collection and storage of every event produced in the collisions at the Tevatron represents a technological problem. The CDF data acquisition system can store data at a maximum rate of 18 $\mathrm{MB} / \mathrm{s}$. With an average event size of $170 \mathrm{kB}$ this corresponds to a maximum rate of $100 \mathrm{~Hz}$. Therefore, in processing the $1.7 \mathrm{MHz}$ of collision data, the CDF trigger system must reject more than $99.99 \%$ of the events.

Searches for the Higgs boson and physics beyond SM are among the main goals of the physics program at the Tevatron. Large amounts of data are required to be able to search for these processes. Therefore, the instantaneous luminosity in the Tevatron has been increased over the design goal. Similar to the relation $R=\sigma \mathcal{L}$ for the rate $R$ of a physics process with a cross section $\sigma$, trigger rates grow with increased luminosity:

$$
R=A+B \mathcal{L}+C \mathcal{L}^{2}+D \mathcal{L}^{3}
$$

The higher powers of luminosity in Eq. 3.1 are mainly caused by two effects: 
- overlapping objects from multiple interactions in the same bunch-crossing, which happens in high luminosity regimes

- luminosity dependent fake objects

High purity triggers, such as those that require presence of high $p_{T}$ muons, usually have $C \sim D \sim 0$. Triggers that are based on the presence of $\notin_{T}$ usually have large growth terms, due to a larger fake $\mathscr{E}_{T}$ in high occupancy events. Low energetic jets that are produced in events with multiple interactions could merge and appear as a single high $E_{T}$ jet. This can cause such events to be accepted by a trigger that requires presence of high $p_{T}$ jets, resulting in trigger rate growth.

In order to maintain high efficiency for interesting signatures while rejecting the majority of unwanted events, a three level trigger system is used at CDF, described in Section 2.4. This architecture allows to select events in steps with successively more complete information at each trigger level. Triggers are designed around specific physics goals, in order to accommodate the broad physics program and to maintain high efficiency. Several triggers were designed at CDF for searches in signatures with energy imbalance in the transverse place, which are introduced below.

\section{1 $\mathbb{E}_{T}$ Triggers at CDF}

As described in Section 3.1, one of the main characteristics of the signal events in this search is the presence of a large imbalance in the transverse energy, which results from decays of $Z$ or $W$ bosons. The decays of the Higgs boson are detected as $b$ quark jets. Therefore, it is natural to chose a trigger, which is most efficient for collecting

events with large missing $E_{T}, \mathbb{E}_{T}$. There are three $\mathbb{E}_{T}$ based triggers implemented at CDF (additional details of these triggers are given in Appendix A):

- MET_BJET: require $\not_{T}>20 \mathrm{GeV}$ and two displaced tracks

- MET45: require $\not \phi_{T}>45 \mathrm{GeV}$ 
- MET35_\&_TWO_JETS: require $\notin_{T}>35 \mathrm{GeV}$ and two clusters with $E_{T}>10$ $\mathrm{GeV}$

The MET_BJET trigger would provide a high purity sample for the Higgs boson search in $\not_{T}$ plus jets signature. However, since the trigger rate for MET_BJET is too high, it has been prescaled ${ }^{1}$ since 2004 , resulting in small integrated luminosity collected with this trigger. Since the Higgs boson production is a very rare process, the small amount of data collected with MET_BJET trigger does not provide a high statistics sample to achieve the required sensitivity.

The MET45 is an inclusive high $\not_{T}$ trigger, and the only requirements are on $\not_{T}$ itself. The threshold on $\mathbb{E}_{T}$ is placed at a high value, in order to keep trigger rate low. Data collected with this trigger is used for searches of physics beyond SM, such as SUSY or Dark Matter, where very large $\not_{T}$ is expected to be produced. However, searches for Higgs boson using the MET45 sample would be inefficient, since the majority of signal events would not pass such a high $\not_{T}$ requirement.

The remaining trigger, MET35_\&_TWO_JETS, yields the highest efficiency (from the triggers available at CDF) for Higgs boson searches in $\notin_{T}$ and jets signature. In this analysis we process data collected by MET35_\&_TWO_JETS trigger, which corresponds to $2.1 \mathrm{fb}^{-1}$ of integrated luminosity.

\subsubsection{MET35_\&_TWO_JETS Trigger}

The events in this analysis were recorded using the missing $E_{T}$ (MET) plus jets trigger path (MET35_\&_TWO_JETS, commonly referred to as the "MET+Jets" trigger). The requirements of the MET+Jets trigger at various trigger levels are listed below:

- MET35_\&_TWO_JETS:

- L1 : Require $\not_{T}>25 \mathrm{GeV}$.

\footnotetext{
${ }^{1} \mathrm{~A}$ trigger is referred to as operating at a prescale with a rate $\mathrm{P}$, if only $1 / \mathrm{P}$ fraction of events are recorded.
} 
- L2 : Two clusters with $E_{T}>10 \mathrm{GeV}$

- L3 : Require $\not_{T}>35 \mathrm{GeV}$.

The MET+Jets trigger has changed along the time in order to accommodate for increasing instantaneous luminosity, while keeping the bandwidth low. The first run collected by the MET35_\&_TWO_JETS trigger was taken on the $22^{\text {nd }}$ of July, 2002. Its definition changed on March 27, 2005, when a requirement of at least one central cluster $(|\eta|<1.1)$ was introduced. Starting from September 2006 this trigger was collecting data only when instantaneous luminosity was below $190 \times 10^{-30} \mathrm{~cm}^{-2} \mathrm{sec}^{-1}$, and from April 2007 the MET35_\&_TWO_JETS trigger was operating with a dynamic prescale $^{2}$.

\subsubsection{Level $1 \not \phi_{T}$ Reconstruction}

The Level 1 trigger system is based on custom electronics designed for fast decision making, and allows the reconstruction basic physics object primitives that can be triggered on. Physical calorimeter towers (Section 2.2.5) are organized into a $24 \times 24$ array of trigger towers in $\eta-\phi$. To reduce the complexity and the processing time, the L1 trigger uses only a 8-bit trigger tower energy information, by dropping the least significant bit and the most significant bit. The transverse projection of the tower energies are calculated with the assumption that the event primary vertex is located at $z=0$, and the missing energy at L1 is calculated as a vector sum of trigger tower pairs. The missing energy at L1 has poor resolution, due to a limited available information and the need to make a fast decision. The $\mathscr{E}_{T}$ calculated by the L1 trigger system is usually underestimated. Therefore, the $\not_{T}$ threshold at L1 is chosen to be as low as possible in order to maximize efficiency.

\footnotetext{
${ }^{2}$ Dynamic prescale: a feedback system that adjusts the prescale of triggers throughout a store according to the total trigger rate.
} 


\subsubsection{Level 2 Jet Reconstruction}

Jets at L2 are reconstructed as "clusters" of energy deposits, using a simple algorithm from Run I. The algorithm finds a "seed" tower (threshold $3 \mathrm{GeV}$ ), then attaches adjacent ("shoulder") towers (threshold $1 \mathrm{GeV}$ ) until no more energetic adjacent towers are found. The cluster position is defined as the seed tower position. While this algorithm worked well at the low instantaneous luminosity of the initial phase of Run II, it become problematic as the Tevatron luminosity increased. The effect of the multiple interactions in high luminosity events significantly increases the detector occupancy and leads to the presence of many energetic towers around the seed towers. As a consequence, large number of energetic towers could be erroneously clustered around a seed into a single jet. Since the MET+Jets trigger requires the presence of at least two clusters in an event, the erroneous merging of separate clusters into a single cluster results in a significant efficiency loss. CDF upgraded the calorimeter trigger in order to solve the problems associated with data-taking in high luminosity regime [71], but the data used in this analysis was collected before the completion of this project.

\subsubsection{Level $3 \not_{T}$ Reconstruction}

The full detector information is available to the L3 trigger system, leading to a much improved reconstruction of physics objects. The entire 10-bit information from the trigger towers is available, leading to an enhanced $\mathbb{F}_{T}$ resolution.

\subsection{Event Preselection}

\subsubsection{Quality selections for $\mathbb{H}_{T}$ analysis}

The critical part of this analysis is the requirement of the presence of $\notin_{T}$ in the

event. As it was described in the previous section, $\mathbb{E}_{T}$ in the event can originate not only from neutrinos, but also from various instrumental/detector effects. These 
effects were studied by the $\mathscr{E}_{T}$ working group at CDF, and a set of selections was recommended to use in all $\not_{T}$-based analysis $\left(\not_{T}\right.$ "clean-up cuts"), to remove the instrumental sources of $\mathbb{E}_{T}$, including beam halo muons. These criteria are described in [72]. Here is a summary of the cuts:

- Pass 1

- At least one central jet with $\left|\eta_{d}\right|<0.9$ and $E_{T}>10 \mathrm{GeV}$ (where $\eta_{d}$ is the detector $\eta$ ).

- Event Electromagnetic Fraction (EEMF):

$$
E E M F=\frac{\sum_{j=1}^{N_{j e t}} E_{T}^{j} \cdot E M F_{j}}{\sum_{j=1}^{N_{j e t}} E_{T}^{j}}>1
$$

where $E M F_{j}$ is the fraction of the jet energy deposited in the electromagnetic calorimeter. Only jets with $E_{T}>10 \mathrm{GeV}$ are considered.

- At least one COT track with $p_{T}>0.5 \mathrm{GeV}$ and one axial super layer with six or more hits on it.

- Pass 2

- Event Charge Fraction (ECHF):

$$
E C H F=\frac{\sum_{j=1}^{N_{j e t}} C H F_{j}}{N_{j e t}}>0.1
$$

where $\mathrm{CHF}_{j}$ is the jet charge fraction which is defined as the ratio of the sum of the $p_{T}$ of the tracks matched to matching to the jet energy $E_{T}$.

- At least one good primary vertex in the event

- Pass 3

- Exclude a geometrical region of the calorimeter, where jets would be mismeasured, called the "chimney". This region hosts cryogenic and instrumental connections to the inner detector. The jets that fall into the chim- 
ney region $\left(\phi=\left(60^{\circ}, 100^{\circ}\right)\right.$ and $\left.\eta=(0.5,1.0)\right)$ are almost certainly mismeasured. Hence, we exclude any event that has a jet with $E_{T}>10 \mathrm{GeV}$ that falls into the chimney region.

- Require that the event primary vertex falls within $|z|<60 \mathrm{~cm}$ of the nominal interaction point at the detector center.

All events that fail any of the above requirements are discarded from the analysis in the initial stages of the analysis.

\subsubsection{Run Selection}

Data collected by CDF experiment is organized into sets, each set being part of a "run" [73]. A run corresponds to a time period during which the detector and beam conditions are stable, and is assigned a unique identifier in the database. Every collision within a run is also assigned a unique identifier, which is stored in the database. Therefore, any collision recorded at CDF can be uniquely identified using its run and event number. Properties or conditions of the detector during each collision are recorded by assigning a quality bit, which can be later retrieved from the database.

If the detector was functional during an entire run or a section of the run, then the "good" bit is set, and the data collected during that period can be used for an analysis. The Data Quality Group at CDF compiles a list of good runs, organized by the various components of the detector. This analysis uses runs with good calorimeters and tracking system since these detectors are used for detecting jets, $b$-tagging and lepton identification.

The amount of the analyzed data is calculated as the sum of the integrated luminosities in each of the good runs. After selecting the good runs, the integrated luminosity used in this analysis is $\mathcal{L}_{\text {int }}=2.1 \mathrm{fb}^{-1}$. 


\subsubsection{Trigger Efficiency}

Events collected by the CDF trigger system are stored and reprocessed by dedicated computer farms "offline". Full reconstruction algorithms can be used at this stage, to precisely reconstruct physics objects, since there is no more need to make a fast decision whether or not to keep an event. Measurements of various objects, such as jet $E_{T}$ or $\notin_{T}$ can be corrected, by taking into account an accurate knowledge of the conditions of various components of the detector and most current calibrations. Some of these corrections were described above (see Sections 3.5.2, 3.5.3 and 3.6.1).

Detailed comparisons with experimental data show that offline quantities provide the most reliable description of the simulated physics processes and the detector response. While the actual event selection at the trigger level is based on online measurements, a major part of all data analysis are performed using the offline quantities, with many backgrounds estimated from simulation. Therefore, in order to estimate the trigger acceptance for simulated physics processes, a trigger efficiency needs to be estimated as a function of offline quantities.

The data collected with MET+Jets trigger was also used in a search for the Higgs boson using $1 \mathrm{fb}^{-1}$ of integrated luminosity [74]. Trigger efficiency was computed from several unbiased datasets, such as data collected with high $p_{T}$ muons (CMUP18) or medium $p_{T}$ inclusive jets (JET20). A high $p_{T}$ inclusive jet sample (JET50), and a sample with $\not_{T}>25 \mathrm{GeV}$ threshold (MET-BCK) $)^{3}$ were used as cross-checks. The jet samples suffer from small statistics, since these events are dominated by dijet production with fake $\mathbb{E}_{T}$. The muon sample is richer in events with real $\mathbb{E}_{T}$ (and is the most similar to our signal), and has sufficient statistics to determine the $\notin_{T}$ efficiency. Trigger efficiencies were calculated for all three levels of CDF trigger, and then parametrized as a function of offline quantities:

- corrected $\notin_{T}$ for L1 and L3 triggers: $\left(\epsilon_{\mathrm{L} 1}\left(\not_{T}\right)\right.$ and $\left.\epsilon_{\mathrm{L} 3}\left(\not_{T}\right)\right)$

\footnotetext{
${ }^{3}$ due to a very high trigger rate, MET-BCK sample is heavily pre-scaled. However, since it is an inclusive sample with a low threshold on $\not_{T}$, it is very useful as a back-up sample for trigger efficiency studies.
} 
- corrected jet $E_{T}$ for L2 trigger: $\left(\epsilon_{\mathrm{L} 2}\left(\right.\right.$ jet $\left.\left.E_{T}\right)\right)$.

One conclusion drawn from the trigger study [74] was that the systematic uncertainties originating from the choice of the trigger samples used in the efficiency calculations are unacceptably large at small $\notin_{T}$ (see Figure 4.3). Therefore, we require every event to have $\mathbb{E}_{T}$ above $50 \mathrm{GeV}$. The efficiency of the L2 trigger was found to be nearly $100 \%$, if the transverse energies $E_{\mathrm{T}}^{J_{1}}$ and $E_{\mathrm{T}}^{J_{2}}$ of the two leading jets ${ }^{4}$ $J_{1}$ and $J_{2}$, satisfy the conditions $E_{T}^{J_{1}}>35 \mathrm{GeV}, E_{T}^{J_{2}}>25 \mathrm{GeV}$. Additionally, it was found that a requirement of $\Delta R(J 1, J 2)>1.0$ was needed $^{5}$, in order to avoid cluster merging at L2. The efficiencies of L1 and L3 trigger were obtained in the region $\mathscr{E}_{T}>50 \mathrm{GeV}$, and parametrized as a function of $\mathbb{E}_{T}$. Thus, the trigger treatment is reduced to a dependence only on one variable: $\mathbb{E}_{T}$. Simulated events are then assigned a weight $\epsilon$, depending on the value $\mathbb{E}_{T}$, according to:

$$
\epsilon=\epsilon_{\mathrm{L} 1}\left(\notin_{T}\right) \times \epsilon_{\mathrm{L} 3}\left(\mathbb{E}_{T}\right)
$$

The trigger efficiency study was recently repeated with $2.1 \mathrm{fb}^{-1}$ [75]. The trigger efficiencies were parametrized using full available dataset. Below is a summary of the trigger efficiencies at L1 and L3 trigger levels, which are used in this analysis.

$$
\begin{aligned}
\epsilon_{\mathrm{L} 1}\left(\mathscr{F}_{T}\right) & =\frac{1.0}{1+\exp \left(\frac{31.2-\not \phi_{T}}{6.6}\right)} \\
\epsilon_{\mathrm{L} 3}\left(\not \phi_{T}\right) & =\frac{1.0}{1+\exp \left(\frac{37.0-\not \phi_{T}}{6.6}\right)}
\end{aligned}
$$

The uncertainty on the trigger efficiency for L1, L2 and L3 combined, was found to be:

$$
\Delta \epsilon / \epsilon\left(\notin_{T}\right)= \begin{cases}0.01 \cdot\left[\frac{80-\not \phi_{T}}{10}\right]+0.25 \cdot\left[\frac{80-\not \not_{T}}{50}\right]^{4} & \text { if } \notin_{T}<80 \mathrm{GeV} \\ 0.00 & \text { if } \not_{T} \geq 80 \mathrm{GeV}\end{cases}
$$

${ }^{4}$ the two jets with the highest transverse energy

${ }^{5} \Delta R(J 1, J 2)$ is the distance between the two leading jets' axis: $\Delta R(J 1, J 2)=\sqrt{(\Delta \phi)^{2}+(\Delta \eta)^{2}}$ 
Trigger efficiencies at L1 and L3, obtained from the studies of control samples are shown in Figures 4.1-4.2. The total trigger efficiencies $\epsilon$ from Eq. 4.4, obtained from different samples, are compared in Fig. 4.3. As can be seen from Fig. 4.3, the systematic variations in the trigger efficiency in the region $\mathbb{E}_{T}<50 \mathrm{GeV}$ are very large. Therefore, in this analysis we require all events to satisfy $\not_{T}>50 \mathrm{GeV}$ requirement, and the events below that threshold are discarded.

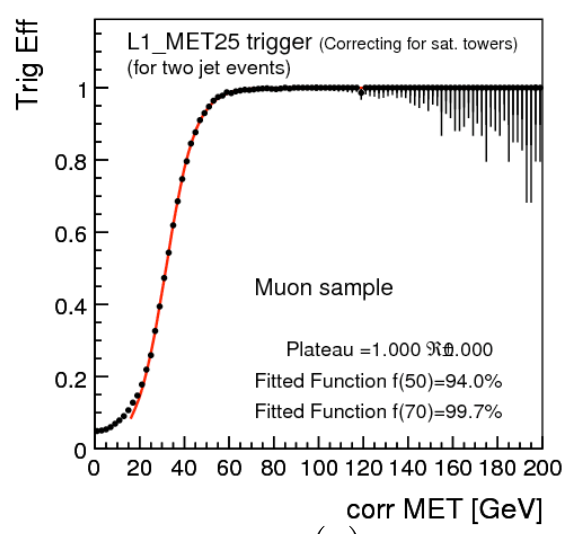

(a)

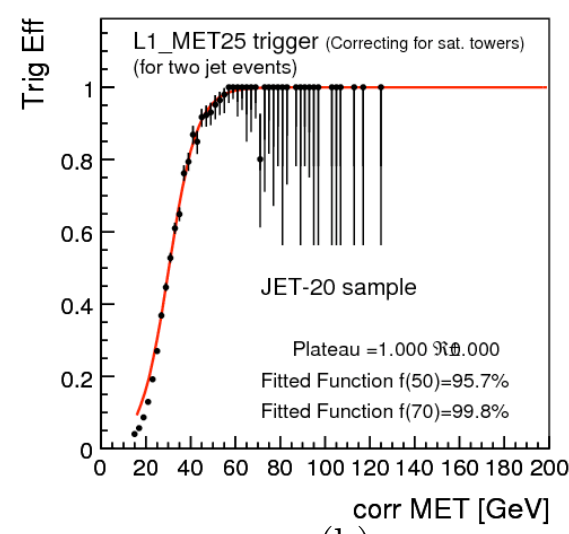

(b)

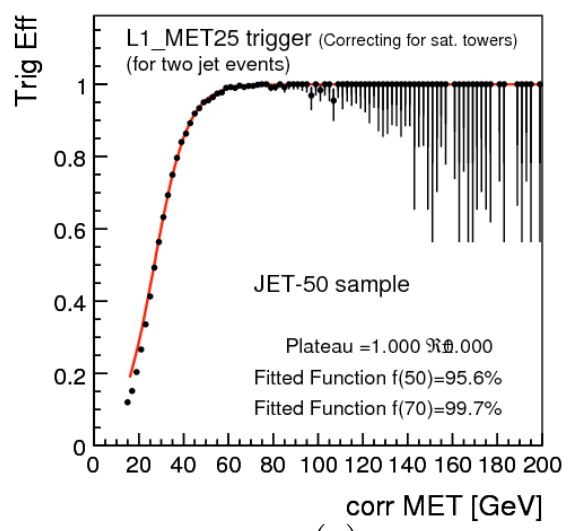

(c)

Figure 4.1.: Efficiency of the L1 MET25 trigger in $2.1 \mathrm{fb}^{-1}$ for (a) CMUP18, (b) JET20, and (c) JET50. Parametrization of the trigger efficiency is shown in red, as a function of corrected $\mathbb{E}_{T}$ 


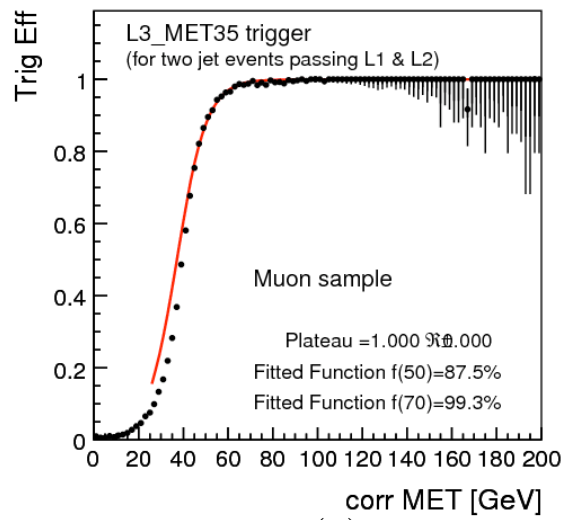

(a)

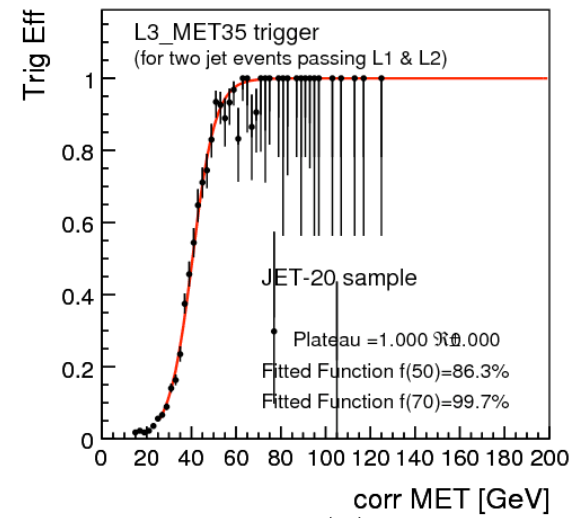

(b)

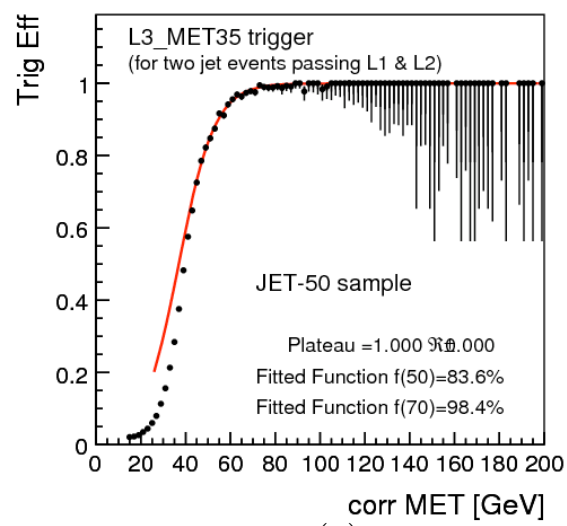

(c)

Figure 4.2.: Efficiency of the L3 MET35 trigger in $2.1 \mathrm{fb}^{-1}$ for (a) CMUP18, (b) JET20, and (c) JET50. Parametrization of the trigger efficiency is shown in red, as a function of corrected $\mathbb{E}_{T}$ 


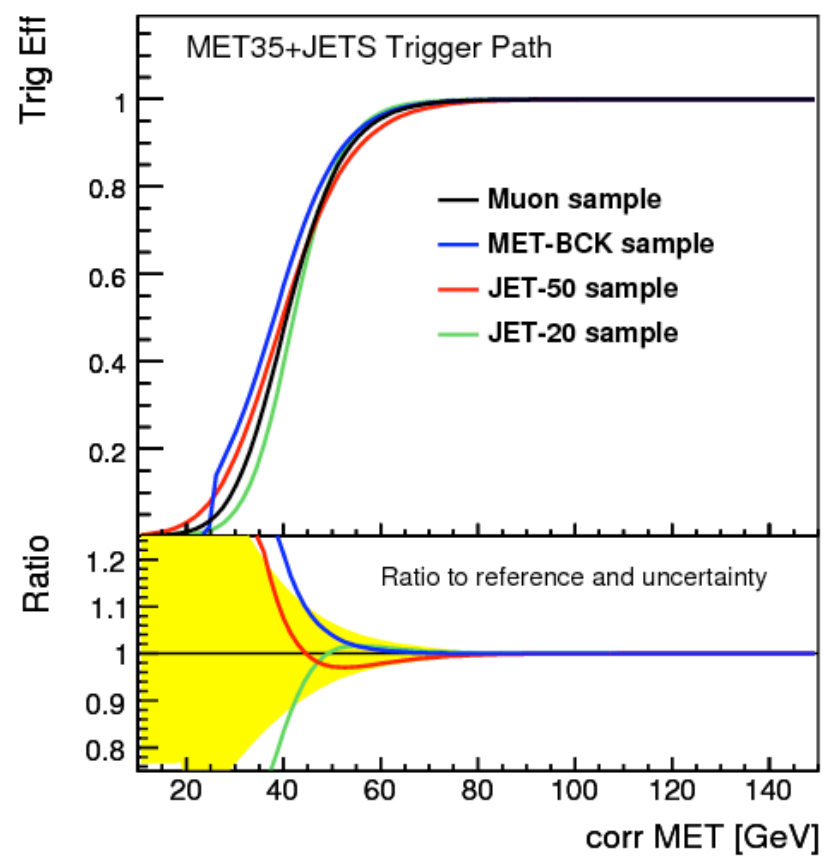

Figure 4.3.: Comparison of total trigger efficiency of the MET+Jets sample, obtained from several samples. The lower plot shows the relative difference from the nominal values. The band indicates the relative uncertainty. 


\subsubsection{Event Selection for the Analysis Sample}

Events passing the trigger-driven requirements described in the previous Section are required to comply with the following additional requirements. We exclude run ranges 217990-220272 and 245448-246231 due to the presence of noisy calorimeter towers in this periods of data-taking. In addition to this, we modify the good-runlist following the instructions from the DQM group and remove a few runs where the information about the beamline position was missing in the database, which is important for reliable $b$-tagging.

In order to reduce the complexity and the processing time, the Level 1 trigger uses only 8-bit trigger tower energy information (Section 4.1.2), resulting in a maximum scale of measured energy in a single tower of $E_{\max }=127 \mathrm{GeV}$. Therefore, if the energy deposited in a single tower at Level 1 exceeds $E_{\text {max }}$, only $127 \mathrm{GeV}$ is used in the $\mathbb{F}_{T}$ calculation. These events should have been automatically accepted, in order to avoid the trigger efficiency loss. However, due to a hardware problem in the $\mathbb{E}_{T}$ calculation at the Level 1 trigger [74], this was not implemented for the dataset used in this analysis. We recover these events by collecting them from the high $p_{T}$ jet sample (JET100), as described in [76], by adding them to the MET+Jets. The JET100 sample is an un-prescaled sample that triggers on a presence of a single jet above $100 \mathrm{GeV}$ (Appendix A). Therefore, the JET100 automatically contains all events with single towers above $E_{\max }$, that should have been collected by the MET+Jets sample.

As described in Section 3.5.1, jets are reconstructed offline using the JetClu algorithm with a cone radius $\mathrm{R}=0.4$. All jets are required to satisfy $|\eta|<2.0$ and uncorrected transverse energy $E_{T}>10 \mathrm{GeV}$. Jet energies are corrected using the generic CDF corrections and the $\mathrm{H} 1$ algorithm (Sections 3.5.2, 3.5.3). Additionally, all jets are required to have H1-corrected $E_{T}>15 \mathrm{GeV}$, otherwise the jet is not considered in the analysis. 
One of the main goals of this analysis was to increase signal acceptance with respect to a previous search in this channel using $1 \mathrm{fb}^{-1}$ of CDF data [74]. The first major change was to loosen the cuts to the following values: $\notin_{T}>50 \mathrm{GeV}$ (was required $\not_{T}>70 \mathrm{GeV}$ ), $E_{T}^{J 1}>35 \mathrm{GeV}$ (was required $E_{T}^{J 1}>60 \mathrm{GeV}$ ) and removing majority of other cuts used in [74].

The second step in increasing the signal acceptance lies in the decision to accept all events with three jets. This allows us to be sensitive to events where one of the $b$-quarks coming from the Higgs radiates a gluon, which is expected to happen around $25 \%$ of the time, according to signal simulation. In addition, $W H$ events where the charged lepton coming from the $W$ is reconstructed as a jet could be reconstructed using three jet events. This could happen in events when $W \rightarrow e \nu$ and the electron fails the CDF electron identification algorithm and is identified as a jet, or when $W \rightarrow \tau \nu$ and $\tau \rightarrow$ hadrons. Table 4.1 shows the contributions in the final signal region from $W H$ processes in 2 and 3 jet events.

Table 4.1: Contributions to 2/3jet events from different decay modes of the $W$-boson in $W H$ events

\begin{tabular}{|l|c|c|c|c|}
\hline Process & \multicolumn{2}{|c|}{ All events } & \multicolumn{2}{c|}{$e, \tau$ matched jet } \\
\hline \hline & 2 jet & 3 jet & 2 jet & 3 jet \\
\hline$W \rightarrow \tau \nu$ & $44 \%$ & $61 \%$ & $2.8 \%$ & $33 \%$ \\
$W \rightarrow e \nu$ & $38 \%$ & $25 \%$ & $0.6 \%$ & $4 \%$ \\
$W \rightarrow \mu \nu$ & $18 \%$ & $14 \%$ & - & - \\
\hline
\end{tabular}

The major drawback of accepting three-jet events lies in the increase of QCD multijet production (a factor of two more) and pair produced top background (a factor of 4 more). In order to reduce these type of backgrounds, events with four or more jets are rejected.

In summary, all events analyzed in this search are required to pass the following set of selections: 
- good run list requiring good calorimetry and good silicon detector during data acquisition (see Section 4.2.2)

- $\notin_{T}$ clean-up cuts to remove instrumental sources of missing energy (see Section 4.2.1)

- $\notin_{T}>50 \mathrm{GeV}$ in order avoid the region of high systematic uncertainty in the trigger efficiency parametrization (see Figure 4.3)

- $\Delta R(J 1, J 2)>1.0$ to avoid cluster merging at L2

- at least two jets with $E_{T}>25 \mathrm{GeV}$ and $|\eta|<2.0$ with at least one of the them having $E_{T}>35 \mathrm{GeV}$ in order to make the L2 trigger fully efficient.

- at least one central jet $|\eta|<0.9$, to allow a unique treatment of various versions of the MET+Jets trigger

- reject events with four or more jets

Events passing all of the above selections are referred to as the "pretag" sample and constitute the analysis sample used in this search.

Additionally, we use $b$-tagging to identify jets coming from $H \rightarrow b \bar{b}$ decays, and to reduce the backgrounds from light flavor $(u, d, s$ quark and gluon) jet production. Only the two leading jets in the event are checked for $b$-tagging. The MET+Jets sample is divided into three independent samples, split by $b$-tagging requirements as the following:

- the event is identified as double tagged $(\mathrm{ST}+\mathrm{ST})$, if the two leading jets in the event are tagged by the Tight SecVtx (ST) algorithm

- if the above fails, but the event contains one jet tagged by Tight SecVtx and another one tagged by Loose Jet Probability (JP) algorithm ${ }^{6}$, the event is identified as double tagged $(\mathrm{ST}+\mathrm{JP})$

\footnotetext{
${ }^{6} \mathrm{~A}$ jet is defined as tagged by Loose Jet Probability if it has less than $5 \%$ probability of coming from the primary vertex
} 
- if both of the above fail, but the event contains one jet tagged by Tight SecVtx algorithm, the event is identified as single tagged (Exclusive ST)

This way, all of the three samples are mutually exclusive and can be combined at the end, to achieve greater sensitivity. 


\section{Chapter 5. Signal and Background Modeling}

The environment is everything that isn't me.

A. Einstein (1879-1955)

A good description of various physics processes that contribute to the MET+Jets data-sample is required in order to predict its features, and be able to detect possible deviations from these predictions in the search of the Higgs boson signal. Unfortunately, a straightforward calculation of experimental observables is usually impossible, due to the complicated nature of hadron collisions and the interactions of the remnants of the collision while passing through the detector material. Computer simulations are commonly used to "generate" particle collisions. Using Monte Carlo techniques, these programs generate events with the distribution predicted by theory, with the same behaviour and the same fluctuations as in real data.

We use Monte-Carlo simulation techniques to estimate the contributions from several of the background processes, as well as the expectation for the Higgs boson signal (simulated samples are commonly referred to as "Monte Carlo samples"). All simulated samples include run-dependent effects, such as the average number of interactions per bunch-crossing during various run-sections, or variations in detector conditions over time. Multiple interactions are simulated by including a number of minimum bias events which is proportional to the average instantaneous luminosity in each run. The major source of the background in this analysis, QCD multijet production, is estimated using data-driven techniques, as described in Section 5.2.1.

\subsection{Signal Modeling}

The signal Monte Carlo samples are generated using PYTHIA [77], with various Higgs boson mass hypotheses, in the range from $105 \mathrm{GeV} / c^{2}$ to $150 \mathrm{GeV} / c^{2}$, in steps 
of $5 \mathrm{GeV} / c^{2}$. While most of the signal acceptance in this analysis comes from $Z H \rightarrow$ $\nu \nu b \bar{b}$ and $W \rightarrow l \nu b \bar{b}$ modes, we also consider events from $Z H \rightarrow \ell \ell b \bar{b}$ channel, where both charged leptons are not identified or escape detection. Table 5.1 lists various mass points at which the signal samples generated. We correct the NLO production cross-sections for NNLO effects by a k-Factor of 0.99 in case of $Z H$ production and 0.96 for $W H$ production [78]. In these samples the Higgs is forced to decay into $b$-jet pairs, the $Z$-boson to neutrinos or charged leptons, and the $W$ decays to leptons. We use $\operatorname{Br}(Z \rightarrow \nu \nu)=0.200, \operatorname{Br}\left(Z \rightarrow l^{+} l^{-}\right)=0.099$ and $\operatorname{Br}(W \rightarrow l \nu)=0.324$.

Table 5.1: The cross-section times branching ratios (number of events produced per $\mathrm{fb}^{-1}$ ) of the $Z H / W H$ processes at various Higgs mass hypothesis

\begin{tabular}{|c|c|c|c|c|}
\hline $\begin{array}{c}M_{H} \\
\left(\mathrm{GeV} / \mathrm{c}^{2}\right)\end{array}$ & $\operatorname{Br}(H \rightarrow b \bar{b})$ & $\begin{array}{c}\sigma_{Z H} \times \operatorname{Br}(\nu \nu, b \bar{b}) \\
(\mathrm{fb})\end{array}$ & $\begin{array}{c}\sigma_{W H} \times \operatorname{Br}(l \nu, b \bar{b}) \\
(\mathrm{fb})\end{array}$ & $\begin{array}{c}\sigma_{Z H} \times B r\left(l^{+} l^{-}, b \bar{b}\right) \\
(\mathrm{fb})\end{array}$ \\
\hline \hline 105 & 0.7957 & 22.777 & 62.708 & 11.275 \\
\hline 110 & 0.7702 & 18.998 & 51.832 & 9.404 \\
\hline 115 & 0.7322 & 15.62 & 42.246 & 7.735 \\
\hline 120 & 0.6789 & 12.573 & 33.693 & 6.223 \\
\hline 125 & 0.6097 & 9.825 & 26.110 & 4.864 \\
\hline 130 & 0.5271 & 7.419 & 19.556 & 3.673 \\
\hline 135 & 0.4362 & 5.374 & 14.047 & 2.660 \\
\hline 140 & 0.3436 & 3.720 & 9.644 & 1.841 \\
\hline 145 & 0.2556 & 2.435 & 6.263 & 1.205 \\
\hline 150 & 0.1757 & 1.475 & 3.765 & 0.730 \\
\hline
\end{tabular}

\subsection{Background Modeling}

As described in Section 3.1, the most important characteristics of the signal events are: the presence of a large $\mathscr{E}_{T}$ from the decays of the $W / Z$ and at least two jets from the Higgs boson decay. Many Standard Model processes can resemble this signature:

- QCD multijet production with mismeasured jets resulting in fake $\not_{T}$ 
- $W / Z$ production with jets and real $\mathbb{E}_{T}$ from $W / Z$ decays

- top quark production with real $\mathbb{E}_{T}$ from $W$ decays, and jets from the top quark decay

- diboson production with real $\mathscr{\phi}_{T}$ from $W / Z$ decays and jets

As can be seen from the above list, the backgrounds in MET+Jets sample can be

divided into two categories: events with real $\mathscr{\phi}_{T}$, and events where $\not_{T}$ originates from mismeasured jets.

In this search we analyze events where at least one of the jets is identified as a $b$ quark jet (Section 4.2.4). Therefore, the goal of the background model is to predict the shapes and event yields of the $b$-tagged sub-sample of the MET+Jets dataset. In this section, we list all the backgrounds considered in the analysis and describe our method to estimate their contributions.

\subsubsection{Multijet Production}

Since we require no identified leptons, the $Z H / W H$ search using the MET+Jets is dominated by QCD multijet production of $b$ - and $c$-quarks (h.f., originating from a $b$ or $c$ quark) and light flavor jets mistakenly identified as $b$-jets (mistags). While these processes generally do not have intrinsic $\mathscr{E}_{T}$, if the energy of one of the jets is mismeasured or a heavy quark decays semi-leptonically, a dijet event can appear to have a large energy imbalance in the transverse plane. While the $\mathscr{E}_{T}$ clean-up cuts reduce a large fraction of events with instrumental $\mathbb{E}_{T}$, it is impossible to completely eliminate them, without a significant loss of signal acceptance. For this reason, this analysis has the additional challenge with respect to the ones with identified leptons, of having much larger, and less-understood, backgrounds. Finally, the lack of reconstructed charged leptons, and the presence of the neutrino leave us with under-constrained kinematics, and thus the impossibility to reconstruct the final state. 
Since the QCD production cross-section is very high $(\sim \mu b)$, it is extremely difficult to generate a Monte-Carlo sample with high $\mathscr{E}_{T}$ and statistics. Additionally, the theoretical uncertainties related to the generation of N-parton events, especially with higher jet multiplicities, make such calculations unreliable. Therefore, for this analysis we developed a method to estimate the QCD background directly from data. The method we use is based on the evaluation of the probability to tag a jet as originating from a heavy flavor quark. This method relies on one of the main characteristics of the QCD events with large fake $\mathbb{E}_{T}$ : the $\vec{\phi}_{T}$ tends to be aligned parallel or antiparallel to one of the jets. This happens both in the case when the missing transverse energy originates from a mismeasured jet, and when a heavy flavor quark decays semi-

leptonically: the $\vec{\notin}_{T}$ points along the under-measured jet, which is usually either the second or third highest energy jet in the event. For signal events the behaviour is different, as shown in Figure 5.1. This characteristic provides the most effective strategy to reduce the QCD background by removing events where $\Delta \phi\left(\vec{E}_{T}\right.$, any jet $)<$ 0.4 from the analysis sample. Additionally, the removed events can be used to study the features of the multijet production and serve as a background model in the final signal region (definition of the signal region is given in Section 8.3). Therefore, we

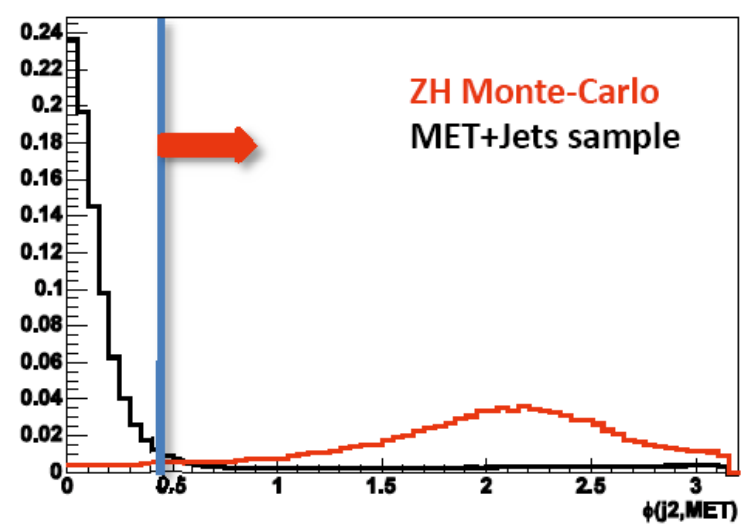

Figure 5.1.: The distribution of $\Delta \phi\left(\overrightarrow{\mathbb{E}_{T}}, \overrightarrow{J_{2}}\right)$ in MET+Jets sample compared to $Z H$ Monte Carlo simulation (the scale is arbitrary). 
split the analysis sample into several regions of interest, as shown in Figure 5.2, based on the value of $\not_{T}$ and $\Delta \phi\left(\vec{\notin}_{T}, J 2\right)$

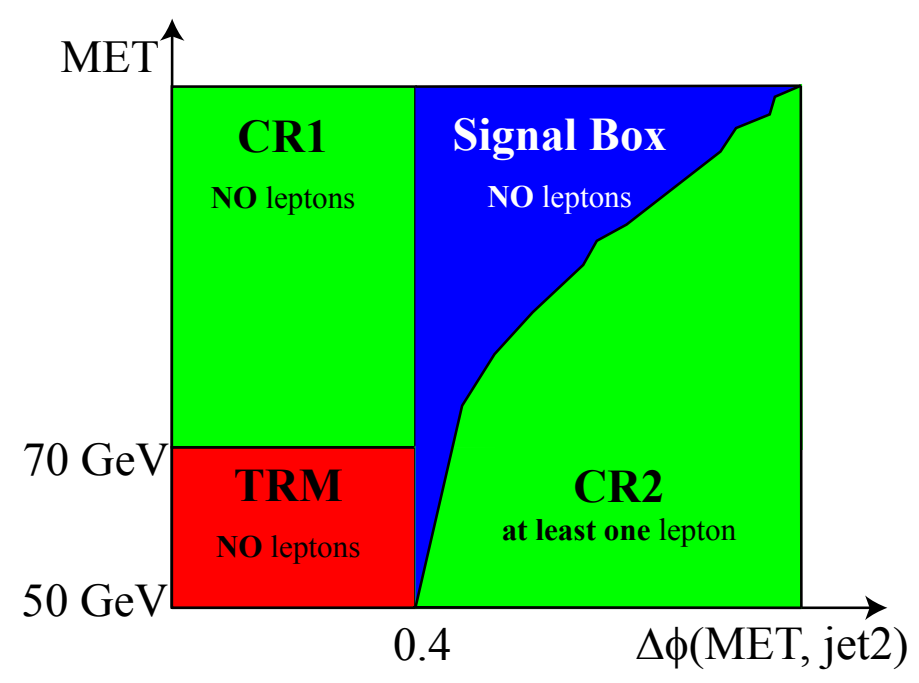

Figure 5.2.: The definitions of the control regions and the TRM region. The line separating the Signal Box and CR2 is for illustrative purpose only. The only difference between these two regions is that we require at lease one charged lepton in CR2, while in Signal Box all events with charged leptons are vetoed.

The jet tagging probabilities are calculated using events depleted in signal, and are used as a measure of the tag rate in events in the signal region. The depleted events are taken to be those with $50 \mathrm{GeV}<\not_{T}<70 \mathrm{GeV}$ and $\Delta \phi\left(\vec{\phi}_{T}, J 2\right)<0.4$. This region is shown in Figure 5.2, labeled as TRM. The requirement of $50 \mathrm{GeV}<\not_{T}<70 \mathrm{GeV}$ is chosen to select a large enough region to calculate the probabilities and to leave the region with $\notin_{T}>70 \mathrm{GeV}$ as a "control region", to test the method. The tag rate per jet is evaluated in this control sample and is parametrized in terms of variables sensitive to both the efficiency for true heavy-flavored objects and the rate of false tags. These variables are: the transverse energy of a jet $E_{T}$; the absolute value of the jet pseudo-rapidity $\eta$; the $H_{T}$ of the event ${ }^{1}$; and the fraction of jet $p_{T}$ carried by

\footnotetext{
${ }^{1}$ event $H_{T}$ is defined as the scalar sum of the transverse energies of all tight jets in the event
} 
charged particles inside the jet (jet fragmentation function $Z$ ). To compute the last quantity, only charged particles satisfying $0.5 \mathrm{GeV} / c<p_{T}<200 \mathrm{GeV} / c$ are used, and the distance of the closest approach to the event primary vertex is required to be less than $5 \mathrm{~cm}$. Jets originating from $b$-quarks are expected to have the highest $Z$-values, followed by $c$-quarks and then light flavour jets, see Figure 5.3. These fourvariable tagging rate parametrization is referred to in the following as the "Tagging Rate Matrix" or TRM, and it allows to predict the contributions from both real heavy flavor jets and from the light flavor mistags.

\section{$\mathrm{ST}+\mathrm{ST}$ Multijet Background Estimation}

In order to predict the shapes of the multijet background in the $\mathrm{ST}+\mathrm{ST}$ sample, we build a Tag Rate Matrix in the TRM region shown on Fig.5.2. The matrix is built in such a way as to predict the rate at which an event that has at least one Tight SecVtx tagged jet will have the other jet Tight SecVtx tagged as well. Each bin in the matrix is then constructed in the TRM region as a ratio of $\mathrm{ST}+\mathrm{ST}$ events to $\mathrm{ST}$

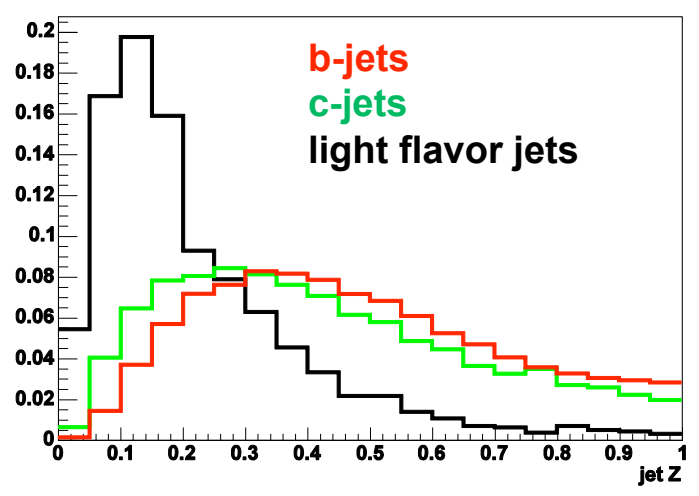

Figure 5.3.: The distributions of jet fragmentation function $Z$ for jets in QCD Monte Carlo simulation (the scale is arbitrary). The red distribution correspond to $b$-quark jets, the green curve to $c$-quark jets and the black curve to light flavor jets. 
events ${ }^{2}$ in that bin. The probability to tag a jet in $i^{\text {th }}$ bin of the matrix is hence calculated by:

$$
R_{i}=\frac{\text { Number of } \mathrm{ST}+\mathrm{ST} \text { events in bin } i}{\text { Number of ST events in bin } i}
$$

The Tag Rate Matrix is implemented by assigning each event in the analysis sample a probability to be double tagged, as a function of the parameters in the matrix. When making a distribution of a particular kinematic variable, each event falling into that distribution is re-weighted according to its tag rate probability $R_{i}$. For the ST+ST sample we use the following procedure:

1) Weight the distribution of events that have the $1^{\text {st }}$ leading jet Tight SecVtx tagged by the probability of the $2^{\text {nd }}$ leading jet $R\left(E_{T, 2}\left|\eta_{2}\right|, H_{T}, Z_{2}\right)$.

2) Weight the distribution of events that have the $2^{\text {nd }}$ leading jet Tight SecVtx tagged by the probability of the $1^{\text {st }}$ leading jet $R\left(E_{T, 1}\left|\eta_{1}\right|, H_{T}, Z_{1}\right)$.

3) Add the results of points 1) and 2) to get the total of ST+ST QCD background.

Fig.5.4 shows projections of matrix along each axis, and Table 5.2 lists the binning used to construct the TRM for ST+ST sample.

Table 5.2: The binning used for the ST+ST Tag Rate Matrix

\begin{tabular}{|c|c|c|c|}
\hline Jet $E_{T}(\mathrm{GeV})$ & Jet $|\eta|$ & Event $H_{T}(\mathrm{GeV})$ & Jet $Z$ \\
\hline \hline 25 & 0.0 & 60 & 0.0 \\
\hline 50 & 0.4 & 110 & 0.1 \\
\hline 80 & 0.8 & 150 & 0.3 \\
\hline 100 & 2.0 & 170 & 0.6 \\
\hline 120 & & 1000 & 10.0 \\
\hline 1000 & & & \\
\hline
\end{tabular}

\footnotetext{
${ }^{2}$ Single Tagged here means $\geq 1$ SecVtx tags, and the non-tagged jet is required to have at least two good Tight SecVtx tracks, i.e. it is Tight SecVtx taggable
} 


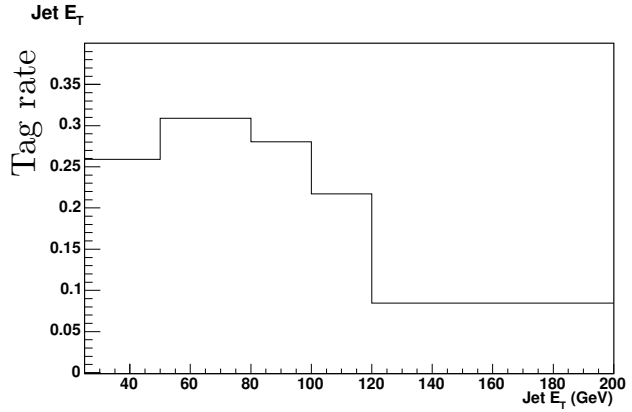

(a)

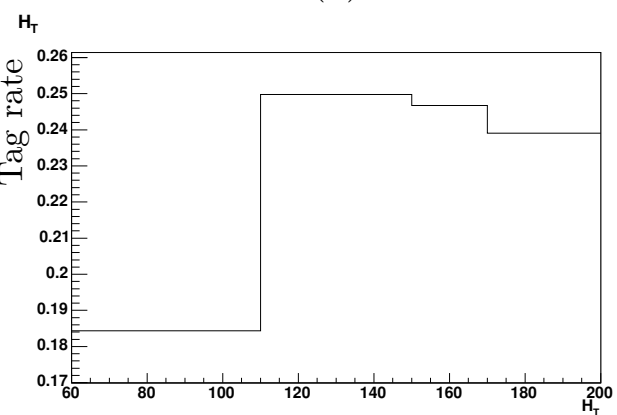

(c)

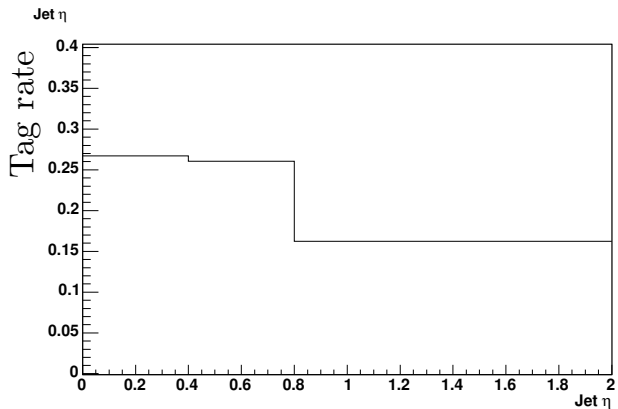

(b)

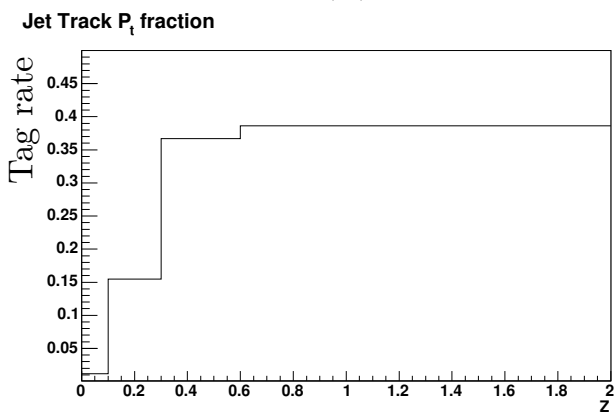

(d)

Figure 5.4.: Tagging probabilities for ST+ST events, as a function of TRM variables: (a) jet $E_{T}$, (b) jet $\eta$, (c) event $H_{T}$, (d) jet $Z$. The distributions are shown using the same bin sizes as in Tab. 5.2 


\section{$\mathrm{ST}+\mathrm{JP}$ QCD Background Estimation}

In order to predict the shapes of ST+JP multijet background we use a similar procedure as described above. We build the TRM matrix in such a way as to predict the rate at which an event that has at least one Tight SecVtx tagged jet will have the other jet Loose JetProb tagged (but not Tight SecVtx tagged).

Each bin in the matrix is constructed as a ratio of Tight SecVtx+Loose JetProb events to Single-Tagged events. The probability to tag a jet in $i^{\text {th }}$ bin of the matrix is hence calculated by Eq.5.2, where the numerator in is composed of events that have only one Tight SecVtx tag, in order to exclude the ST+ST sample.

$$
R_{i}=\frac{\text { Number of ST }+\mathrm{JP} \text { events in bin } i}{\text { Number of ST events in bin } i}
$$

We use the same weighting procedure to obtain the distributions in ST+JP as the one described for $\mathrm{ST}+\mathrm{ST}$ in Section 5.2.1.

Fig.5.5 shows projections of ST+JP matrix along each axis. We used the same binning for the ST+JP matrix as for the $\mathrm{ST}+\mathrm{ST}$ one. 


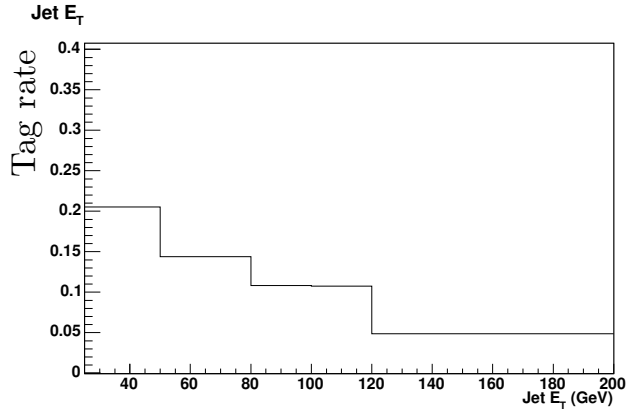

(a)

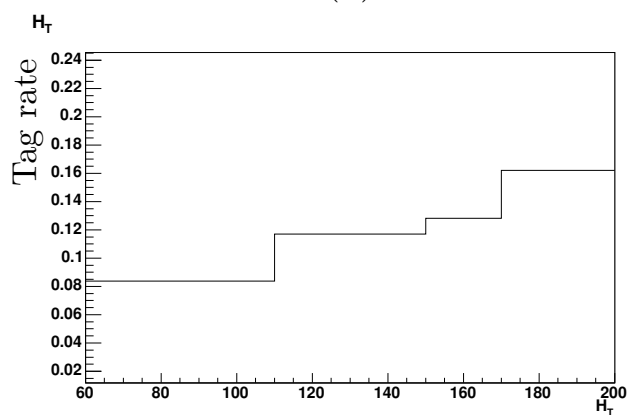

(c)

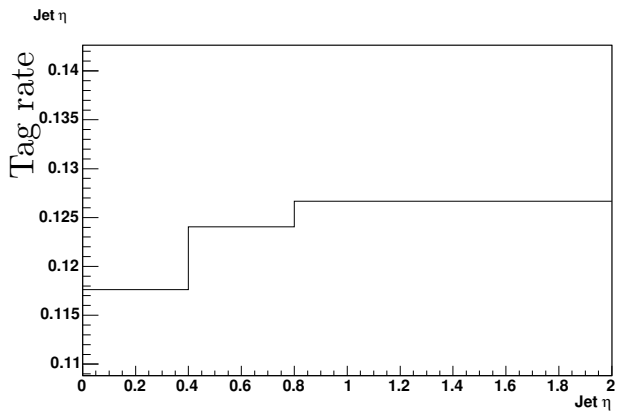

(b)

Jet Track $P_{t}$ fraction

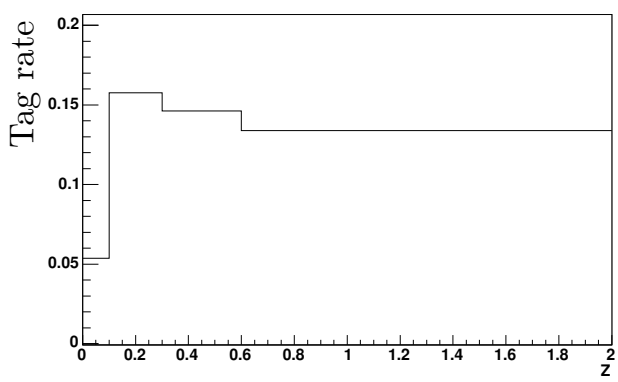

(d)

Figure 5.5.: Tagging probabilities for ST+JP events, as a function of TRM variables: (a) jet $E_{T}$, (b) jet $\eta$, (c) event $H_{T}$, (d) jet $Z$. The distributions are shown using the same bin sizes as in Tab. 5.2 


\section{Exclusive ST QCD Background Estimation}

The TRM matrix to predict the single-tagged multijet background is built in such a way as to predict the rate at which a taggable jet ${ }^{3}$ in the pretag sample is tagged by Tight SecVtx algorithm, and the other jet in the event is not tagged by neither Tight SecVtx nor Loose JetProb.

Each bin in the matrix is constructed as a ratio of ST events to taggable events falling into that bin. The probability to tag a jet in $i^{\text {th }}$ bin of the matrix is calculated using Eq.5.3. The numerator in Eq.5.3 is composed of events that have only one Tight SecVtx tag, and the other jet is not tagged by SecVtx Tight or Loose JetProb.

$$
R_{i}=\frac{\text { Number of Exclusive ST events in bin } i}{\text { Number of pretag events in bin } i}
$$

We use the following procedure to weight the events to predict single-tagged multijet background.

1) Weight the pretag distribution by the probability to tag the $1^{\text {st }}$ leading jet and not the $2^{\text {nd }}, R\left(E_{T}^{J_{1}}\left|\eta_{1}\right|, H_{T}, Z_{1}\right)$.

2) Weight the pretag distribution by the probability to tag the $2^{\text {nd }}$ leading jet and not the $1^{s t}, R\left(E_{T}^{J_{2}}\left|\eta_{2}\right|, H_{T}, Z_{2}\right)$.

3) Add the results of 1) and 2)

Fig.5.6 shows projections of matrix along each axis, and Table 5.3 lists the binning used to construct the TRM for Exclusive ST sample.

\footnotetext{
${ }^{3} \mathrm{~A}$ jet is considered taggable if there are at least two good Tight SecVtx tracks in the 0.4 cone around jet axis
} 


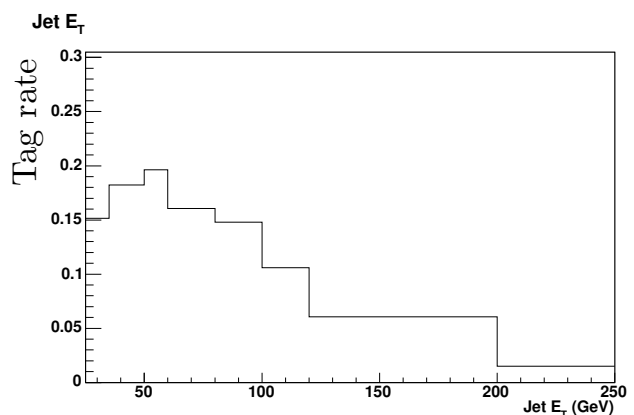

(a)

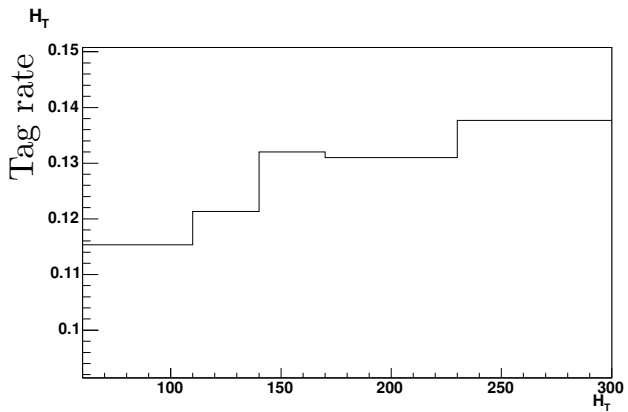

(c)

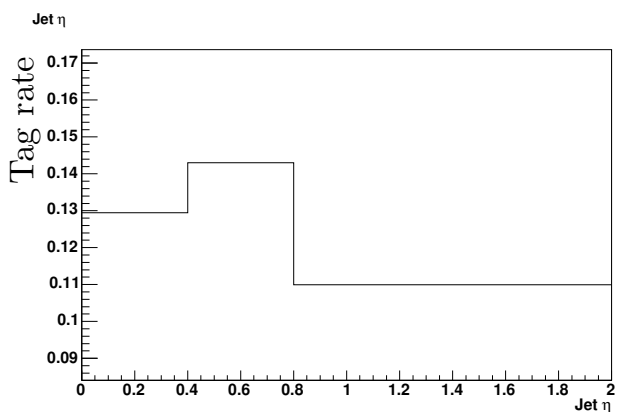

(b)

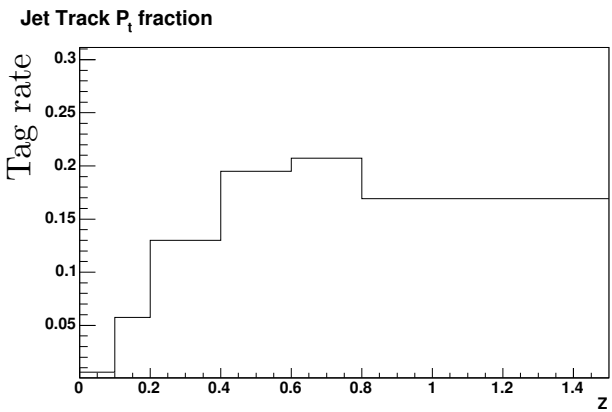

(d)

Figure 5.6.: Tagging probabilities for Exclusive ST events, as a function of TRM variables: (a) jet $E_{T}$, (b) jet $\eta$, (c) event $H_{T}$, (d) jet $Z$. The distributions are shown using the same bin sizes as in Tab. 5.2

Table 5.3: The binning used for the Exclusive ST Tag Rate Matrix

\begin{tabular}{|c|c|c|c|}
\hline Jet $E_{T}$ & Jet $|\eta|$ & Event $H_{T}$ & Jet $Z$ \\
\hline \hline 25 & 0.0 & 60 & 0.0 \\
\hline 35 & 0.4 & 110 & 0.1 \\
\hline 50 & 0.8 & 140 & 0.2 \\
\hline 60 & 2.0 & 170 & 0.4 \\
\hline 80 & & 230 & 0.6 \\
\hline 100 & & 1000 & 0.8 \\
\hline 120 & & & 10.0 \\
\hline 200 & & & \\
\hline 1000 & & & \\
\hline
\end{tabular}




\section{Removing the Double Counted Events from Multijet Estimation}

In this analysis, the events with with real $\mathbb{E}_{T}$ that contribute to the MET+Jets sample are estimated using Monte Carlo simulations. However, when the TRM probabilities are applied to the real data, the non-QCD processes will also contribute, resulting in double-counting events from the same physics process. To account for this, we use the following procedure:

1) first, the matrix is applied to the real data, as described above

2) the same matrix is applied to the non-QCD backgrounds (Monte-Carlo predictions), to obtain the shapes and yields of the double counted events from non-QCD backgrounds.

3) subtract point 2) from 1)

\subsubsection{Di-boson and $W / Z$ backgrounds}

The electroweak backgrounds were all generated with PYTHIA. All the $W+$ jets and $Z+$ jets samples were filtered for a $b$ - or $c$-quark at generator level with the exception of $W \rightarrow \tau \nu$ and $Z \rightarrow \tau \bar{\tau}$. In some cases, the events were also $\not_{T}$ filtered, after the event reconstruction whenever it resulted in a significant reduction of the event number. The leading order PYтніA Monte Carlo predictions for $W+$ jets, $Z+$ jets and diboson production $(W W, W Z$, and $Z Z)$ are scaled by a factor of $1.4[79,80,81]$ to account for higher order effects.

\subsubsection{Top Production}

We consider two classes of top-quark production in this analysis: the pair-production and the electroweak production of single top quark in the t- and s-channels. They all yield a measurable contribution to the background in the signal region, but the 
pair-production is the most significant. Due to its large mass and the semi-leptonic decay of the top, these events are energetic, have large $\not_{T}$ and high jet multiplicity.

We estimate the top quark pair production using PYTHIA simulation, while for single top production we use MADEVENT [82] program. The $t \bar{t}$ events were generated with a top quark mass of $175 \mathrm{GeV} / c^{2}$ and normalized to the measured 7.0 $\pm 0.6 \mathrm{pb}[83]$ cross section. The single top events are normalized to theoretical production cross sections $0.88 \pm 0.14 \mathrm{pb}$ and $1.98 \pm 0.3 \mathrm{pb}$ in the s-channel and t-channel, respectively $[84,85]$. 


\section{Chapter 6. Control Regions and Signal Box}

The man of science has learned to believe in justification, not by faith, but by verification.

Thomas Henry Huxley (1825-1895)

In this analysis, we are searching for a signal of Higgs boson production in events with $\not_{T}$ and jets. The SM prediction of the cross-sections in $W H / Z H$ modes is below $0.1-0.2 \mathrm{pb}$, which is orders of magnitude smaller than the production crosssection of the backgrounds that are present in this channel. In order to confirm that the background modeling is robust and to avoid potential bias in the search, we test our understanding of the SM backgrounds in several "control" regions that are depleted of signal events and are defined a priori. The events in the kinematic region where the signal is expected to be produced are excluded from the analysis, until the analysis method, selection cuts, background estimates and systematic uncertainties are fixed (the "blind signal box"). We choose the size of the signal box to be larger than the signal region, so that the final signal cuts may be chosen without bias. When a good agreement is achieved in the control regions, i.e. the experimental data agrees with our model within the assigned uncertainties, we perform a final optimization of the final signal region based only on signal and background simulation (at this stage the data in the signal box is still hidden). At the end, the signal box is "opened", and the comparison of observed data and background prediction is made. If no significant difference is found, a 95\% confidence level upper limit is set on Higgs boson production cross-section.

After the event preselection described in Section 4.2, the data sample is mainly composed of multijet production (QCD+light flavour mistagged jets) processes. The high $\not_{T}$ in these events comes both from mismeasurement of the jets energies, which 
causes the $\overrightarrow{E_{T}}$ to point along one of the jets, and from real sources of $\mathbb{E}_{T}$ such as neutrinos or muons from semi-leptonic b-decays. We use a subset of the MET+Jets sample to derive a model for these types of events (Section 5.2.1) which will be used in the final signal region, defined in Sec. 8.3. We build three control regions, which we will use to test separately our data-driven and simulation-based background modeling.

\subsection{Definitions}

In order to test our ability to predict the multijet background, we check the performance of the TRM method in two control regions. The first, control region 1 (CR1), is a high statistics region where we check the data-based model and evaluate the systematic uncertainties on the shapes of various kinematic variables.

Since in the signal region we expect backgrounds originating from events with real high $\notin_{T}$, such as $W / Z+$ jets, $t \bar{t}$, single top production and diboson production, we test our ability to predict these types of backgrounds in another region, control region 2 (CR2). In order to remain unbiased to our signal region, we test electroweak/top backgrounds in the kinematic region similar to signal region, with the exception of requiring at least one lepton in the event (all events with leptons are vetoed in the signal region definition). CR2 is sensitive to electroweak/top processes, and is used to check the shapes of the Monte Carlo predictions. CR2 also serves as an additional (but low statistics) check of the multijet model. The double-tagged sample in CR2 is rich in top pair production, allowing an additional cross-check of this background.

The second QCD control region (CR3) will be defined in Section 7.3.

In summary, the following selections define the signal and control regions:

- Control Region 1 (QCD dominated)

- All leptons are vetoed using the lepton identifications described in Section 3.8).

- Azimuthal angular separation $\Delta \phi\left(\overrightarrow{J 2}, \overrightarrow{\notin_{T}}\right) \leq 0.4$ 
- $\notin_{T}>70 \mathrm{GeV}\left(50 \mathrm{GeV}<\notin_{T}<70 \mathrm{GeV}\right.$ region is used to build the TRM)

- Control Region 2 (EWK/Top processes)

- Minimum 1 loose lepton is required

- Azimuthal angular separation $\Delta \phi\left(\vec{J} 2, \overrightarrow{\mathscr{E}_{T}}\right)>0.4$

- Signal Box

$-\not_{T}>50 \mathrm{GeV}$

- All leptons are vetoed

$-\Delta \phi\left(\vec{J} 1, \overrightarrow{\notin_{T}}\right) \geq 1.5, \Delta \phi\left(\vec{J} 2, \overrightarrow{\notin_{T}}\right) \geq 0.4$ and $\Delta \phi\left(\vec{J} 3, \overrightarrow{\notin_{T}}\right) \geq 0.4$

\subsection{Background Model Comparison with Data in Control Regions}

In order to estimate the backgrounds originating from QCD heavy flavor multijet production and incorrectly tagged light flavor jet production, we use the Tag Rate Matrix (TRM) method, described in Section 5.2.1. This method provides an excellent model describing the shapes of the observables very well. Nonetheless, the number of expected multijet events is not predicted well enough, as was observed in several control regions, and needs to be adjusted by a scale factor. In order to constrain the expected rates of the multijet background, we utilize the CR2 and CR3, which are kinematic regions very close to the signal region, as described in Section 7.4. In this section, we test our capability of reproducing the shapes of the observed distributions in data in CR1 and CR2. In these two regions, the multijet prediction is normalized to $N_{o b s}-N_{M C}$, where $N_{o b s}$ is the number of observed events and $N_{M C}$ is the number of expected events from MC simulation. Once we are confident that the shapes are well reproduced by the matrix, we extract the normalization factor, and use this normalization in the final measurement.

Tables 6.1, 6.2 and 6.3 list the expected and observed background yields in all control regions for all tagging categories. We use the control regions to cross-check 
the modelling of the shapes of kinematic distributions. Comparisons of kinematic distributions in control regions are shown in Figures 6.1-6.6. Additional comparisons are shown in the Appendix B. The observed data is modeled very well both in multijet enriched region CR1 where the background prediction us derived using the TRM method, and in CR2 where the background prediction is derived using MC simulated samples.

It should be noted, that the dijet mass distribution in CR1 is shifted to higher values compared to CR2. This is due to a fact that in CR1 the $\not_{T}$ originates from mismeasurements of jets' energy. By requiring the $E_{T}^{J_{2}}>25 \mathrm{GeV}$ and $\not_{T}>50 \mathrm{GeV}$ we bias the $E_{T}^{J_{1}}$ toward higher values, which results in higher values of dijet mass. Another feature of events with mismeasured jets can be observed in the distribution of $\Delta \phi\left(\overrightarrow{\not_{T}^{t r}}, \overrightarrow{E_{T}}\right)$. In events with real $\mathscr{E}_{T}$ the calorimeter based $\mathscr{E}_{T}$ calculation and the $P_{T}^{t r}$ calculation based on the tracking system are highly correlated and point in the same direction. In events with fake $\mathscr{E}_{T}$, such as those in CR1, these two quantities are not correlated, and can be pointing in the opposite directions. Figures 6.1-6.6 show that our background prediction provides an adequate model for these effects.

An additional cross-check was performed to ensure that the number of observed jets is modelled well in CR2 by the MC simulation. The jet multiplicity is sensitive to various next-to-leading order effects, which may be difficult to properly estimate using MC simulation, and could potentially provide a source of mis-modelling. Since this analysis was the first one to analyze events with three jets, it was also crucial to check if our background model describes the properties of the third leading jet properly. As can be seen from Figures 6.1-6.6, our background model provides an excellent description of the observed jet multiplicities and the transverse energy distributions of the third jet.

The most important kinematic variable in the searches for $H \rightarrow b \bar{b}$ decays is the invariant mass of the dijet system $M_{j j}$, which allows to reconstruct the Higgs boson resonance. Therefore, it is very important to have a good modelling of this distribution. The comparisons in control regions show that our background model provides 
a good description of $M_{j j}$, both in the events dominated by multijet production, and in events with real $\not_{T}$.

Topological variables, such as relative angles between jets and between jets and $\not_{T}$ and $\not P_{T}^{t r}$ direction provide additional handles in separating signal from background events, and as can be seen from the Figures6.1-6.6, these variables are also wellmodelled.

Since we intend to use Artificial Neural Networks (ANN) to discriminate signal from backgrounds, it is crucial to check that all of the input variables in data are well described by the background model. Additionally, it is important to check that the correlations between individual variables are well reproduced. Since neural networks exploit the correlations between kinematic variables, if those are not well described by the predictions, the ANN output might be unreliable or biased. We perform the correlations checks based on Pearson's correlation coefficients, defined as:

$$
r(X Y)=\frac{\left(X_{i}-\bar{X}\right)\left(Y_{i}-\bar{Y}\right)}{\sigma_{X} \cdot \sigma_{Y}}
$$

where $\bar{X}$ is the average value of variable $X$ and $\sigma_{X}$ is its standard deviation.

The comparisons of correlation coefficients are shown in the Appendix C. Figures C.1-C.6 show the comparison plots of Pearsons's coefficients between data and background model in control regions. As can be seen from these comparisons, the expected and observed correlation coefficients agree with each other.

After performing the checks in numerous control regions and testing the modelling of all variables of interest, we are confident that our background model provides an excellent description of the data. Events with instrumental sources of large $\notin_{T}$ (CR1) and events with real $\mathbb{E}_{T}(\mathrm{CR} 2)$ are very well-described by the prediction. Furthermore, a complete study of the modelling of the correlations between kinematic variables was performed, and a good agreement with data is observed. Therefore, we proceed to optimizing the definition of the signal region, where we will perform the search for the Higgs boson signal. The signal region optimization procedure is described in Section 7. 
Table 6.1: Number of expected and observed events in control regions in Exclusive ST events. The expected Higgs boson signal is shown for $m_{H}=115 \mathrm{GeV} / c^{2}$. The uncertainties contain both Monte Carlo statistical error and systematic uncertainties.

\begin{tabular}{|l|c|c|}
\hline Process & Control Region 1 & Control Region 2 \\
\hline \hline QCD + Mistags & $8751.7 \pm 95.4$ & $449 \pm 24.4$ \\
Single Top & $1.1 \pm 0.2$ & $46.8 \pm 8.6$ \\
Top Pair & $7.2 \pm 1.1$ & $211.8 \pm 28.7$ \\
Di-boson & $0.9 \pm 0.4$ & $24.7 \pm 5.0$ \\
W + h.f. & $20.5 \pm 10.4$ & $148.0 \pm 65.2$ \\
Z + h.f. & $25.6 \pm 12.2$ & $27.7 \pm 13.4$ \\
\hline Observed & 8807 & 908 \\
\hline \hline$Z H \rightarrow \nu \nu b b$ & 0.03 & 0.02 \\
\hline$W H \rightarrow(l) \nu b b$ & 0.08 & 2.07 \\
\hline$Z H \rightarrow(l l) b b$ & 0.01 & 0.37 \\
\hline
\end{tabular}

Table 6.2: Number of expected and observed events in control regions in ST+ST events. The expected Higgs boson signal is shown for $m_{H}=115 \mathrm{GeV} / c^{2}$. The uncertainties contain both Monte Carlo statistical error and systematic uncertainties.

\begin{tabular}{|l|c|c|}
\hline Process & Control Region 1 & Control Region 2 \\
\hline \hline QCD + Mistags & $596.2 \pm 22$. & $19.5 \pm 7.5$ \\
Single Top & $0.3 \pm 0.1$ & $9.3 \pm 1.8$ \\
Top Pair & $1.6 \pm 0.3$ & $63.9 \pm 10.0$ \\
Di-boson & $0.04 \pm 0.04$ & $2.4 \pm 0.7$ \\
W + h.f. & $0.5 \pm 0.5$ & $10.1 \pm 5.2$ \\
Z + h.f. & $3.4 \pm 1.8$ & $1.7 \pm 1.0$ \\
\hline Observed & 602 & 107 \\
\hline \hline$Z H \rightarrow \nu \nu b b$ & 0.01 & 0.01 \\
\hline$W H \rightarrow(l) \nu b b$ & 0.01 & 1.01 \\
\hline$Z H \rightarrow(l l) b b$ & 0.0 & 0.19 \\
\hline
\end{tabular}


Table 6.3: Number of expected and observed events in control regions in ST+JP events. The expected Higgs boson signal is shown for $m_{H}=115 \mathrm{GeV} / c^{2}$. The uncertainties contain both Monte Carlo statistical error and systematic uncertainties.

\begin{tabular}{|l|c|c|}
\hline Process & Control Region 1 & Control Region 2 \\
\hline \hline QCD + Mistags & $1308.3 \pm 36.3$ & $42.7 \pm 10.3$ \\
Single Top & $0.3 \pm 0.1$ & $7.8 \pm 1.6$ \\
Top Pair & $2.0 \pm 0.4$ & $57.9 \pm 9.7$ \\
Di-boson & $0.1 \pm 0.1$ & $2.4 \pm 0.6$ \\
W + h.f. & $1.6 \pm 1.1$ & $11.6 \pm 5.9$ \\
Z + h.f. & $3.7 \pm 2.0$ & $2.7 \pm 1.5$ \\
\hline \hline Observed & 1316 & 125 \\
\hline \hline$Z H \rightarrow \nu \nu b b$ & 0.01 & 0.01 \\
\hline$W H \rightarrow(l) \nu b b$ & 0.02 & 0.85 \\
\hline$Z H \rightarrow(l l) b b$ & 0.0 & 0.15 \\
\hline
\end{tabular}



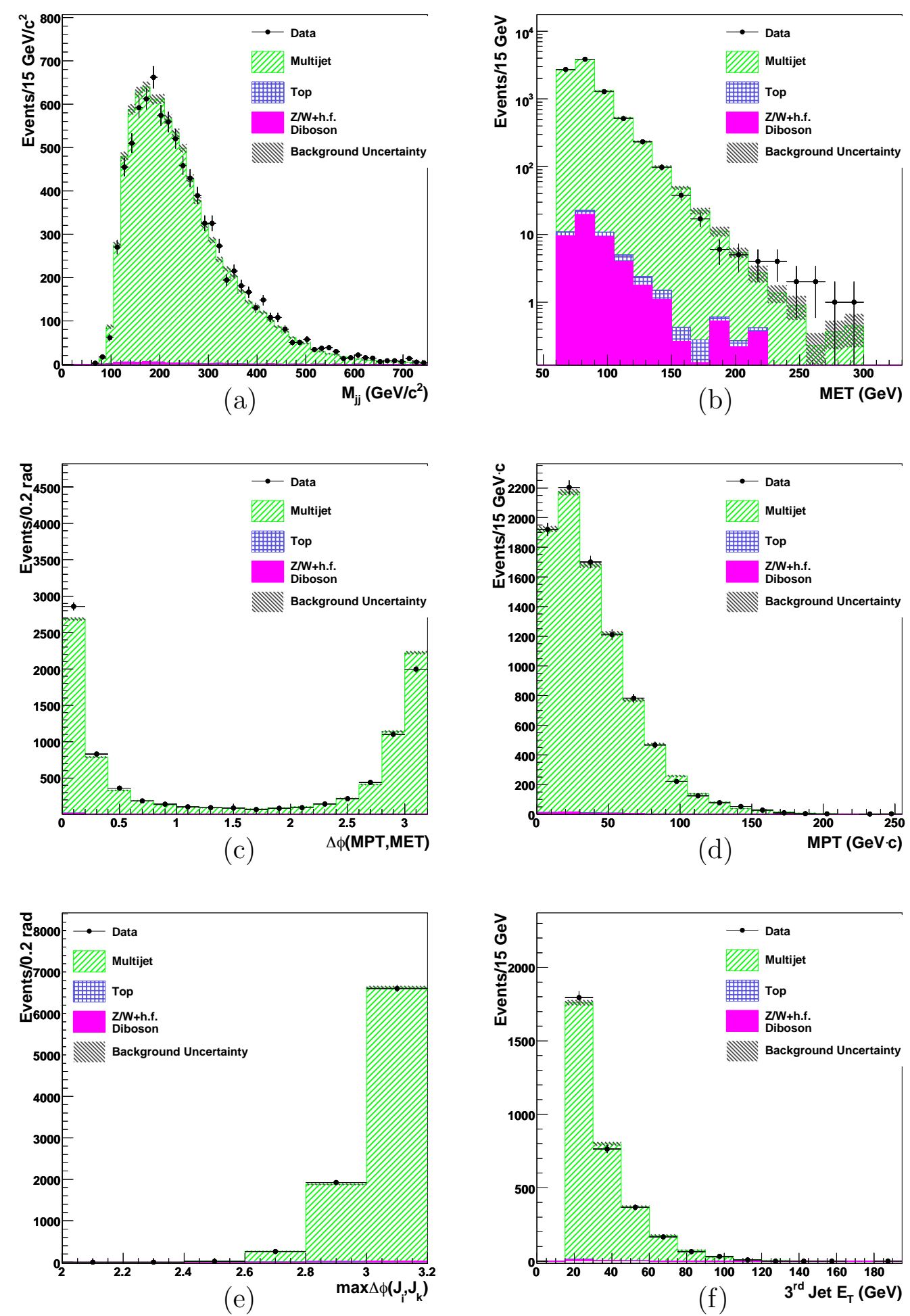

Figure 6.1.: Kinematic distributions in QCD CR1 for events with single b-tag: (a) dijet invariant mass, (b) event $\not_{T}$, (c) $\Delta \phi\left(\overrightarrow{P_{T}^{t r}}, \overrightarrow{\mathbb{E}_{T}}\right)$, (d) event $\not P_{T}^{t r}$, (e) maximum of the difference in $\phi$ between two jets directions, (f) transverse energy of the $3^{\text {rd }}$ jet 

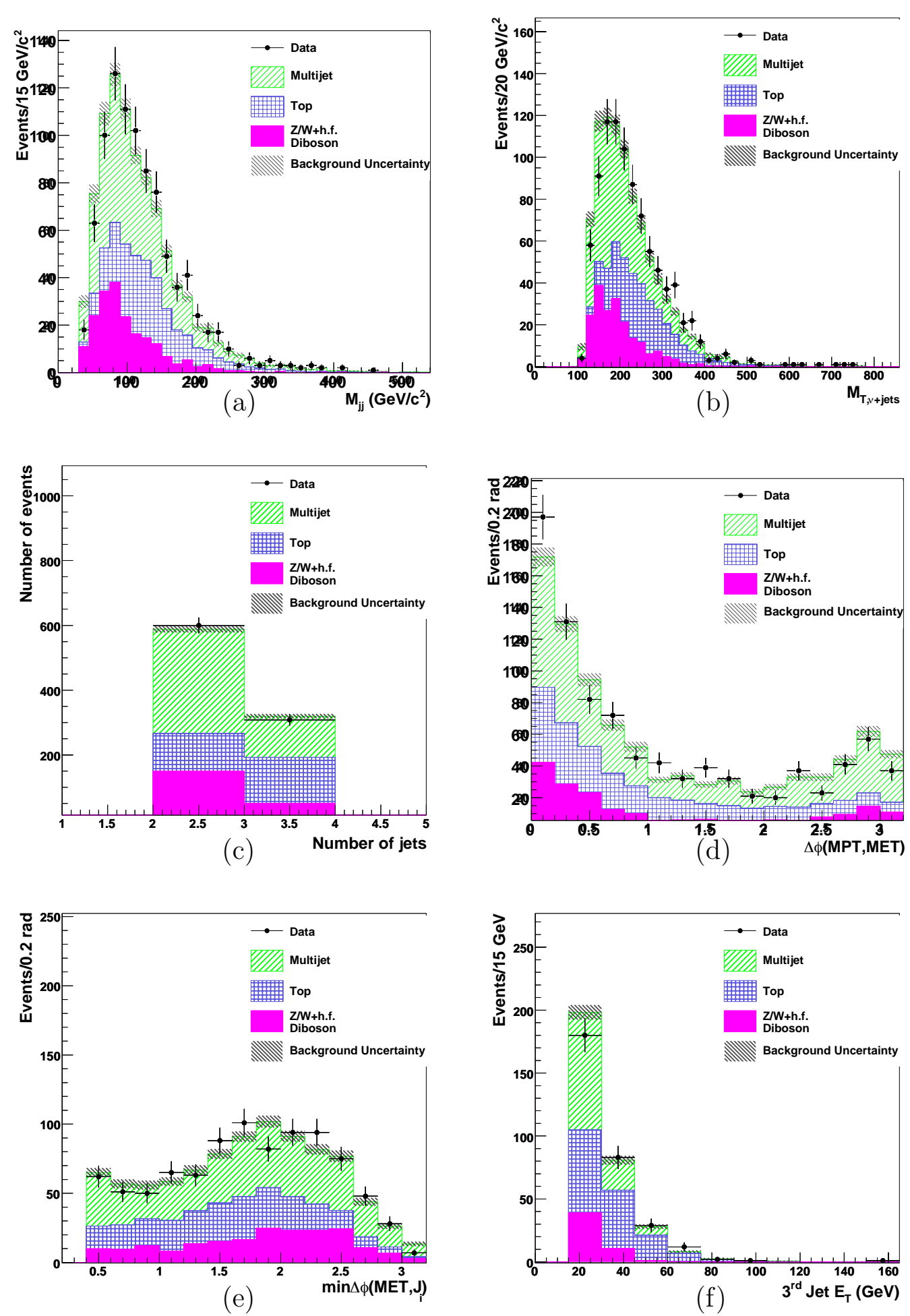

Figure 6.2.: Kinematic distributions in CR2 for events with single $b$-tag: (a ) dijet invariant mass, (b) invariant mass of $\mathscr{E}_{T}$ and all jets, (c) number of jets, (d) $\Delta \phi\left(\overrightarrow{\not_{T}^{t r}}, \overrightarrow{\mathscr{E}_{T}}\right)$, (e) minimum of the difference in $\phi$ between the missing transverse energy and each jet, (f) transverse energy of the $3^{\text {rd }}$ jet 

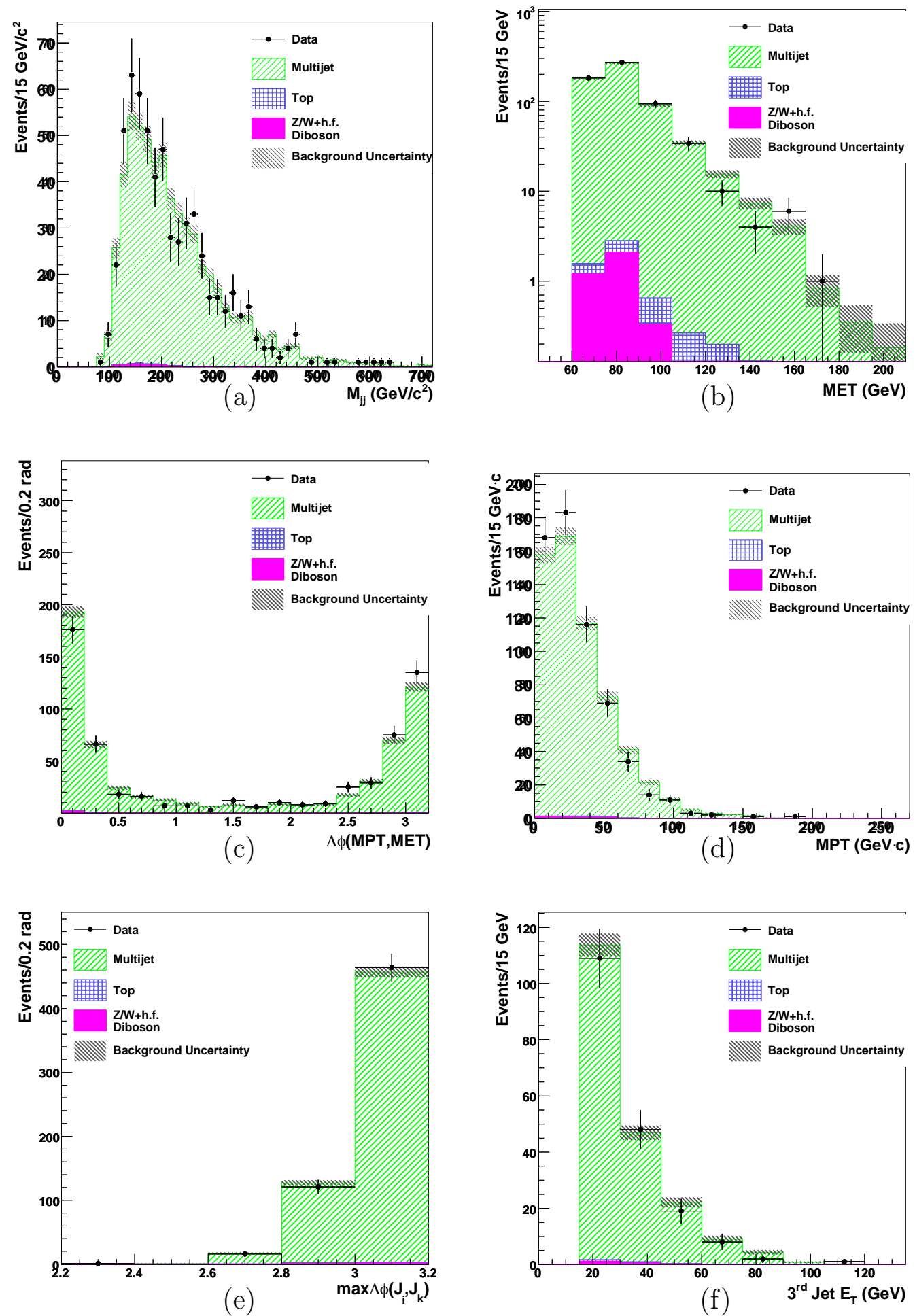

Figure 6.3.: Kinematic distributions in QCD CR1 for ST+ST events: (a) dijet invariant mass, (b) event $\not_{T}^{t}$, (c) $\Delta \phi\left(\overrightarrow{\phi_{T}^{t r}}, \overrightarrow{\phi_{T}}\right)$, (d) event $\not_{T}^{t r}$, (e) maximum of the difference in $\phi$ between two jets directions, (f) transverse energy of the $3^{r d}$ jet 

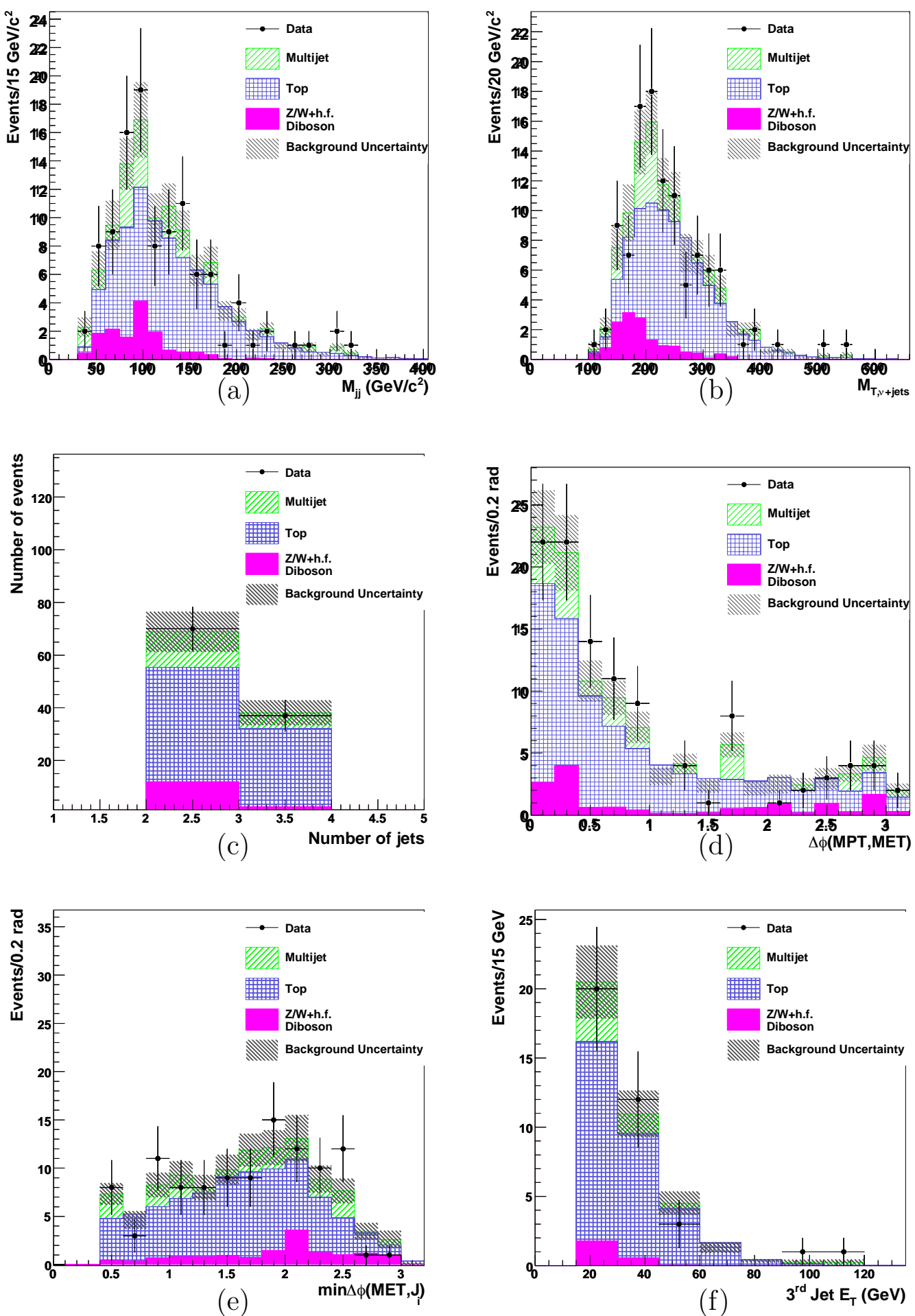

Figure 6.4.: Kinematic distributions in CR2 for ST+ST events: (a ) dijet invariant mass, (b) invariant mass of $\mathscr{E}_{T}$ and all jets, (c) number of jets, (d) $\Delta \phi\left(\overrightarrow{\not p_{T}^{t r}}, \overrightarrow{\mathbb{E}_{T}}\right)$, (e) minimum of the difference in $\phi$ between the missing transverse energy and each jet, (f) transverse energy of the $3^{\text {rd }}$ jet 

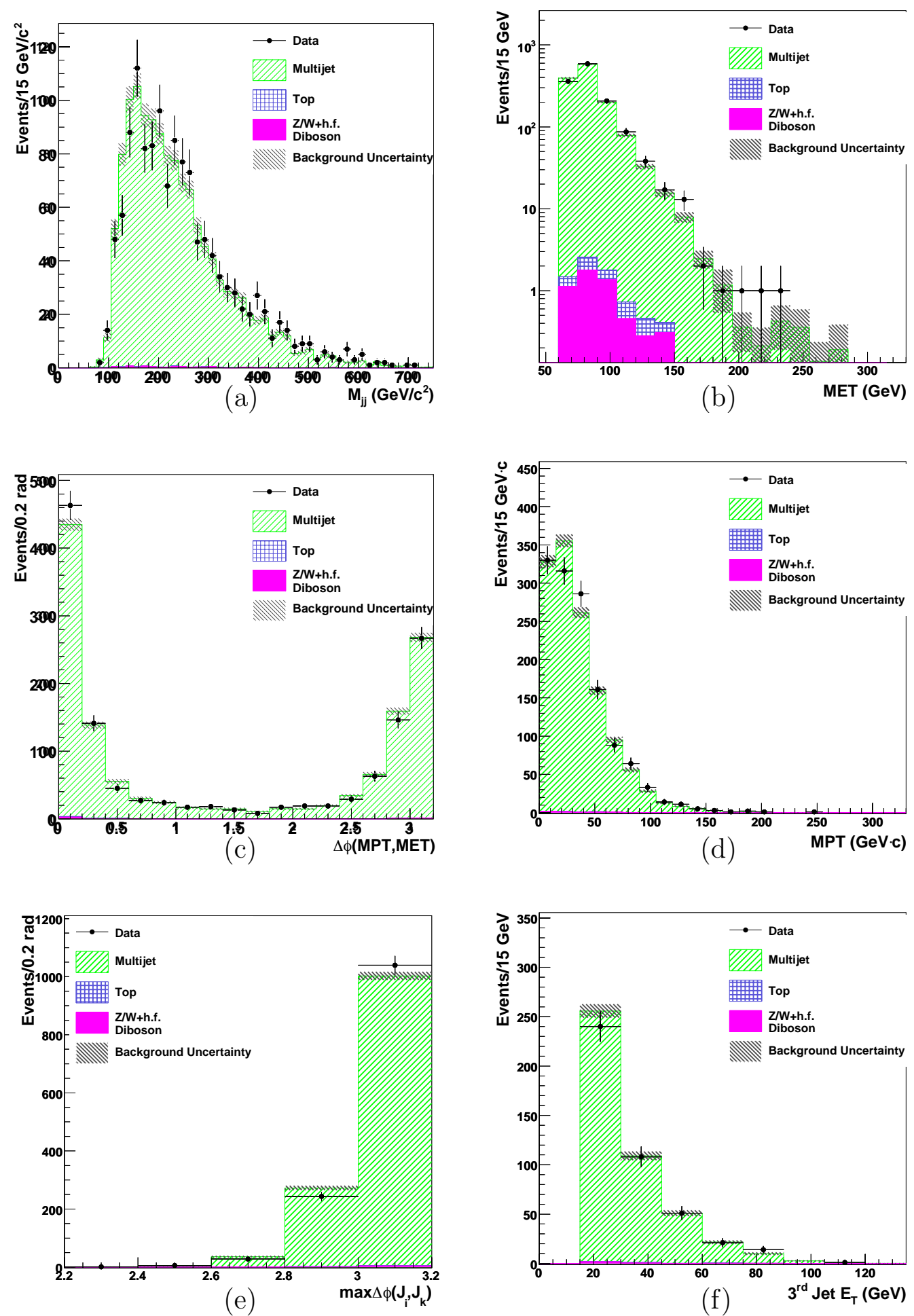

Figure 6.5.: Kinematic distributions in QCD CR1 for ST+JP events: (a) dijet invariant mass, (b) event $\mathscr{E}_{T}$, (c) $\Delta \phi\left(\overrightarrow{P_{T}^{t r}}, \overrightarrow{\phi_{T}}\right)$, (d) event $\not P_{T}^{t r}$, (e) maximum of the difference in $\phi$ between two jets directions, (f) transverse energy of the $3^{\text {rd }}$ jet 

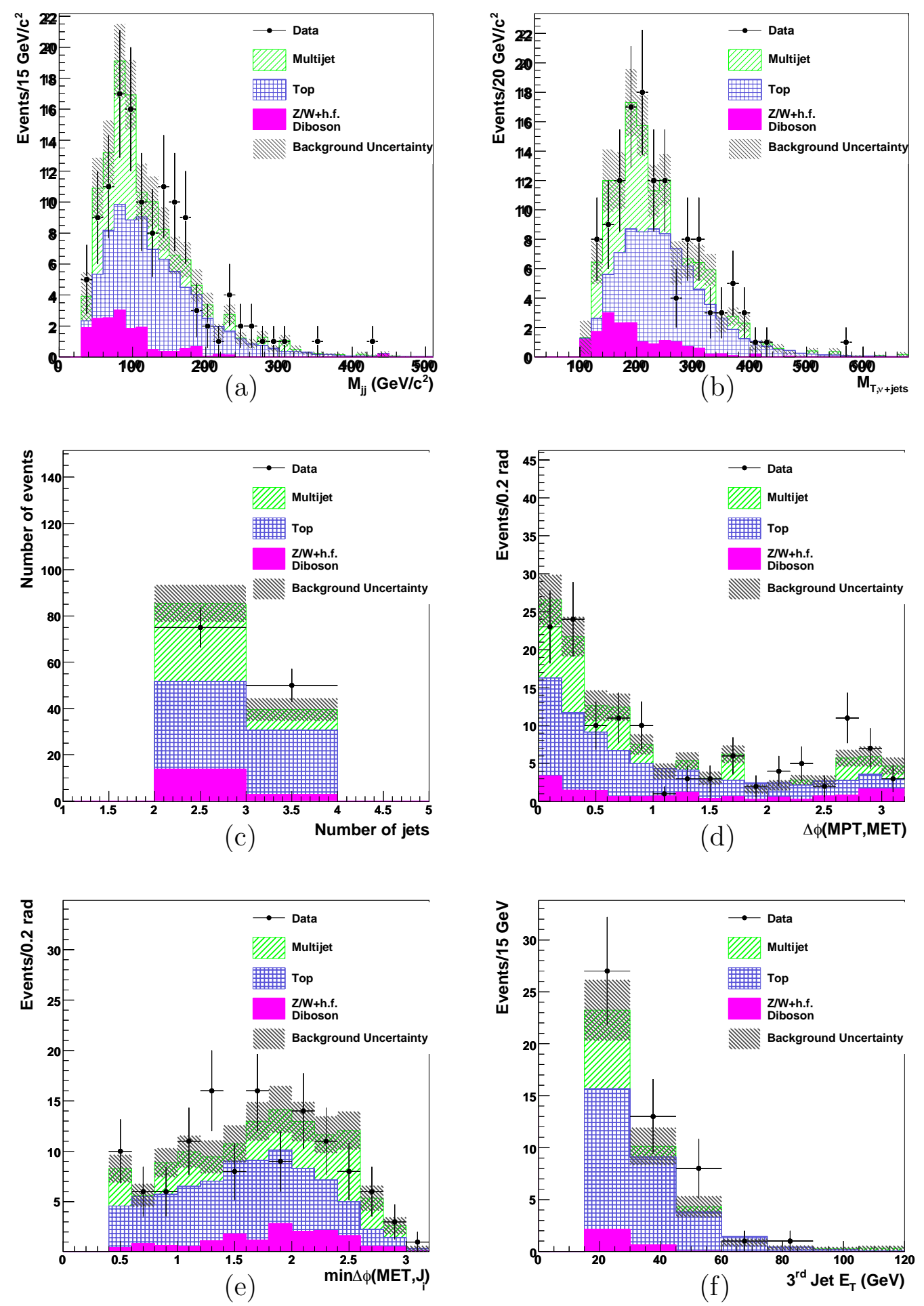

Figure 6.6.: Kinematic distributions in CR2 for ST+JP events: (a ) dijet invariant mass, (b) invariant mass of $\not_{T}$ and all jets, (c) number of jets, (d) $\Delta \phi\left(\overrightarrow{\not p_{T}^{t r}}, \overrightarrow{\mathscr{E}_{T}}\right)$, (e) minimum of the difference in $\phi$ between the missing transverse energy and each jet, (f) transverse energy of the $3^{\text {rd }}$ jet 


\section{Chapter 7. Signal Region Optimization}

As it was stressed before, the main background at this stage of the selection is the QCD production of two or three jets. In order to increase the sensitivity of the search, a substantial improvement of signal to background ratio S/B is needed. Orthogonal selection cuts were used in the past to achieve this goal. This has the disadvantage of losing a large amount of signal together with backgrounds. In order to increase the statistical power of the search, we developed a new method to significantly reduce the amount of backgrounds using artificial neural networks (ANN), with negligible loss of signal events. We exploit distinguishing features of the multijet production of heavy flavor jets in order to construct a single discriminant with the best separating power.

To study the production of QCD heavy flavor jets, we utilize a QCD Monte Carlo simulation of $b \bar{b} / c \bar{c}$ production. We investigate the dynamics of these events by looking at a large set of variables, and select the ones for which QCD heavy flavor production has a very different behaviour with respect to the signal and the remaining backgrounds. The Monte Carlo simulation of QCD events is used only for selection optimization purposes, while the background model of QCD production is estimated using the TRM model described in Section 5.2.1.

\subsection{Statistical Learning Methods}

The classic approach in high-energy physics of signal region selection based on orthogonal cuts has the advantage of being easy to understand and describe. However, the final criteria do not make optimal use of the information available in the event. Usually, even in a simple analysis, tens of variables of interest are studied. Therefore, the space of all possible cuts on all event observables is enormous. There 
can be correlations among those observables, which can be different for signal and backgrounds, that are ignored in a cut based selection.

Multivariate approaches to separating signal from background allow to overcome the drawbacks of cut-based methods, taking advantage of as much of the existing information as possible. In such approaches, one combines various observables into a single one, called a "discriminant", on which a selection to separate signal from background is applied.

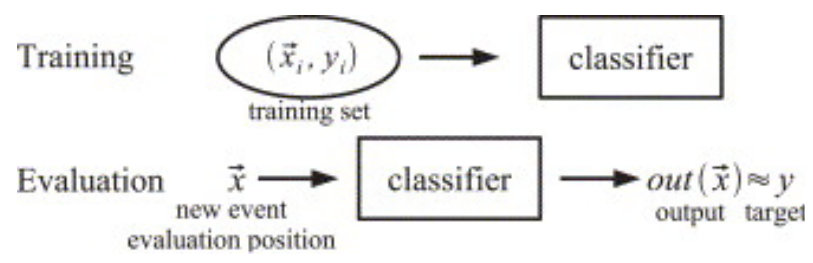

Figure 7.1.: The training of a statistical learning method, that builds a model (classifier) which is then used to evaluate new events.

As was already discussed above, the exact mathematical dependence of the measured observables on the quantity of interest are not known, and an exhaustive search of many variables is the only possibility to find the correct dependence. To compensate for the lack of precise knowledge of these dependencies, statistical learning methods are often used. Statistical learning means that a set of $N$ input variables $\vec{x}_{i}$ and target values $y_{i}$ are given as an input to the learning method. The input variables are experimentally measured quantities, such as jet transverse energies or the event $\mathscr{E}_{T}$, and the target values are usually chosen to be such that they maximally separate the signal events from background events, e.g. the target for a background event is -1 and for a signal event: +1 . The learning method then tries to infer the output function $\operatorname{OUT}(\mathrm{x})$, given the input variables and the target values (this process is called "training"). This process is sketched in Figure 7.1. Statistical learning methods try to model the functional dependence of $\operatorname{OUT}(\mathrm{x})$ by studying background and signal Monte Carlo samples. Afterwards, the output function $\mathrm{OUT}(\mathrm{x})$ can be applied to 
the events in the real data, in order to categorise them as either background-like or signal-like.

An important prerequisite of statistical learning is the availability of training samples. As the term "statistical" implies, the learning method needs to infer the output function from a subsample of the data, and this function is later applied to the total data set. To avoid potential biases, the available Monte Carlo samples are usually divided into training and testing parts. The training part is used to "train" the learning algorithm and the test part is only used during the evaluation of the already trained classifier. During training, the outputs of a learning method approximate the target values given the inputs in the training set, trying to "generalize" the output function, i.e. to have the outputs of the method approximate target values given inputs that are not in the training set. Otherwise, especially if the number of events in the training sample is very small, it may occur that the learning method finds an appropriate output function without achieving the needed generalisation. This is dangerous, since by memorizing peculiar features and fluctuations of the training set, this output function is only valid for the training sample, which is often classified as "overtraining". An estimate of the efficiency of the classifier can be tested in an unbiased manner, by splitting the sample into training and testing parts.

Various multivariate techniques have been developed over the years, based on different mathematical models that describe the relationship between input and output function. One of the most widely used statistical learning methods is the Artificial Neural Network, which is the method used in this analysis.

\subsubsection{Artificial Neural Networks}

Historically, the Artificial Neural Networks (ANN) were inspired by the desire to produce artificial systems capable of sophisticated, "intelligent" computations similar to those that the human brain routinely performs [86]. Some ANNs are models of biological neural networks, consisting of a network of simple processing elements 
("neurons" or "nodes"), that can exhibit complex behavior, determined by the connections between the nodes and their parameters. A neuron has several input and output connections and the weighted sum of all signals received by a neuron generates its response through a non-linear threshold function. During the training, the strengths of the connections between neurons are adjusted on the basis of training sample, to achieve the desired outcome. In other words, ANNs "learn" from examples. Therefore, ANNs exhibit a capability for generalization beyond the training data, and produce correct results for new cases that were not used for training. In this analysis we use a type of ANNs called MultiLayer Perceptron (MLP), which is briefly described below (see [87] for a more detailed description).

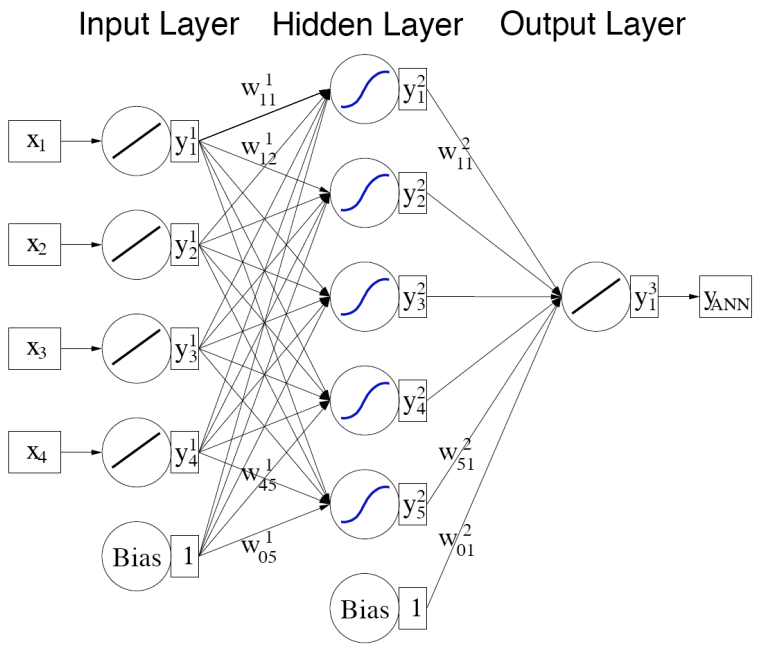

Figure 7.2.: Architecture of a one-hidden-layer feed-forward ANN [88].

The set of $N_{v a r}$ input variables, which are used to discriminate the signal from backgrounds, are passed into the ANN through the "input" nodes $x_{i}$ of the "input layer" (Figure 7.2). Each of the $j$ nodes in the "hidden layer" receives a set of inputs $x_{i}$ from the input nodes and calculates a weighted sum:

$$
y_{i}=\sum_{i=1}^{N_{v a r}} x_{i} w_{i j}^{(1)}
$$


where $w_{i j}^{(1)}$ are the weights assigned to the $i^{\text {th }}$ quantity entering the $j^{\text {th }}$ node of the hidden layer. This type of neural networks, where there is no connection among nodes of the same layer, are called "feed-forward". The response of the hidden nodes is modeled by an "activation function", which in this analysis is chosen to be a hyperbolic tangent: $\tanh (\xi)=\left(e^{\xi}-e^{-\xi}\right) /\left(e^{\xi}+e^{-\xi}\right)$. The goal of the activation function is to model the firing of biological neurons in the brain. The weights associated with the bias node serve as a threshold of firing a particular node. The response of the output node is then given by:

$$
y_{A N N}=\sum_{j=1}^{N_{\text {hidden }}} y_{j}^{(2)} w_{j 1}^{(2)}=\sum_{j=1}^{N_{\text {hidden }}} \tanh \left(\sum_{i=1}^{N_{\text {var }}} x_{i} w_{i j}^{(1)}\right) w_{j 1}^{(2)}
$$

where $w_{j 1}^{(2)}$ are the weights between the hidden layer nodes and the output node, and $N_{\text {hidden }}$ is the number of nodes in the hidden layer.

For each training event $a$ the neural network output $y_{A N N, a}$ is computed and compared to the target output $\hat{y}_{a}$. An error function $E$, measuring the agreement of the network response with the target, is defined by:

$$
E=\sum_{a=1}^{N} \frac{1}{2}\left(y_{A N N, a}-\hat{y}_{a}\right)^{2}
$$

where $\mathrm{N}$ is the total number of events in the training sample. The set of weights that minimizes the error function can be found using an iterative procedure, until a minimum is found, at which point the ANN training is said to have "converged".

\subsection{Multijet Rejection Neural Network}

Past versions of analysis in the MET+Jets sample employed cut based selections [74] in order to reduce the overwhelming background from QCD multijet events with mismeasured jets. In order to keep the QCD background small, $\notin_{T}$ was required to be greater than $70 \mathrm{GeV}$. To increase the signal acceptance as much as possible, we required $\mathbb{E}_{T}>50 \mathrm{GeV}$ in this analysis. In order to reduce the amount of backgrounds, we employ a neural network to select the signal region and to reject the majority 
of events with fake $\mathbb{E}_{T}$. The neural network takes advantage of several topological features that distinguish signal events with real neutrinos from fake $\mathbb{F}_{T}$ events in QCD multijet production.

As a neural network algorithm we chose the MultiLayer Perceptron (MLP), which is a feed-forward network described in Section 7.1. We use the implementation provided by the TMVA package [88]. We use pretag QCD MC events as a background in the training sample, since the QCD Monte Carlo sample that we use for training of this ANN was filtered for the presence of $b / c$ quarks. Hence using the pretag sample allows us to exploit the features of heavy flavour production, while retaining high statistics. The integrated luminosity of the QCD Monte Carlo sample corresponds to $3.2 \mathrm{fb}^{-1}$. The Higgs signal used for the training is a mixture of $50 \% \mathrm{WH}$ events and $50 \% \mathrm{ZH}$ events. Of the total signal and background events, half was used as training sample and the rest as testing sample.

The kinematic variables used as inputs for the ANN training are:

- Absolute amount of the missing transverse energy measured in the calorimeter, $\notin_{T}: M E T$ (Section 3.6)

- Absolute amount of the missing transverse momentum measured in the tracking system, $\not P_{T}^{t r}:$ MPT (Section 3.6.2)

- Maximum of the difference in phi between two jets directions, taking two jets at the time: $\max \left(\Delta \phi\left(J_{i}, J_{k}\right)\right)$

- Maximum of the difference in the $\mathrm{R}$ space between two jets, taking two jets at the time: $\max \left(\Delta R\left(J_{i}, J_{k}\right)\right)$

- Minimum of the difference in $\phi$ between the missing transverse energy and each jet, considering all two or three $\left(\mathbb{E}_{T}, J_{i}\right)$ pairings: $\min \left(\Delta \phi\left(M E T, J_{i}\right)\right)$

- Minimum of the difference in $\phi$ between the $\not T_{T}^{t r}$ and the jets, considering all two or three $\left(\not P_{T}^{t r}, J_{i}\right)$ pairings: $\min \left(\Delta \phi\left(M P T, J_{i}\right)\right)$ 
- Scalar sum of transverse energy of the leading jets, $H_{T}: H T$

- Ratio of missing $H_{T}{ }^{1}$ and missing transverse energy $\not_{T}: M H T / M E T$

- Difference in $\phi$ between missing transverse energy $\not_{T}$ and missing transverse momentum $\not{ }_{T}^{\text {tr }}: \Delta \phi(M E T, M P T)$

The shapes of distributions for the signal and background training samples are shown in Fig. 7.3. The correlation patterns are shown in Appendix in Fig. D.2. It can be seen that the kinematic variables and the correlations between them are very different for the signal and the QCD background. The dijet invariant mass $M_{j j}$, which is the most discriminating variable, is not used in the training of multijet rejection ANN, in order not to bias the $M_{j j}$ distribution.

The topology of the QCD rejection ANN consists of 9 input nodes +1 bias node, 1 hidden layer with 20 hidden nodes, a second hidden layer with 10 hidden nodes, and 1 output node. The number of training epochs ${ }^{2}$ is set to 1000 . We refer to the output of this neural network as the $\mathrm{ANN}_{\mathrm{MJ}}$ in the following.

\section{$\mathrm{ANN}_{\mathrm{MJ}}$ Output and the Choice of Cut Level}

In order to check for ANN overtraining (Section 7.1), a comparison is made between the shapes of the $\mathrm{ANN}_{\mathrm{MJ}}$ output distributions for the testing and training samples, for both signal and background distributions. This comparison is shown in Figure 7.4(a). In the absence of overtraining, the shapes of these distributions must be the same. Statistical compatibility of the ANN distributions is quantified by performing a Kolmogorov-Smirnov (KS) test [89]. The KS test returns a probability that the histograms that are being compared follow the same distribution. The KS probabilities of the $\mathrm{ANN}_{\mathrm{MJ}}$ output for signal and background distributions are shown in the Figure 7.4(a), and are equal to 0.88 and 0.92 for the signal and background

\footnotetext{
${ }^{1}$ Missing $H_{T}(M H T)$ is defined as the absolute amount of the vector sum of all jets' energies

${ }^{2} \mathrm{An}$ epoch is one pass through all the records in the training set.
} 

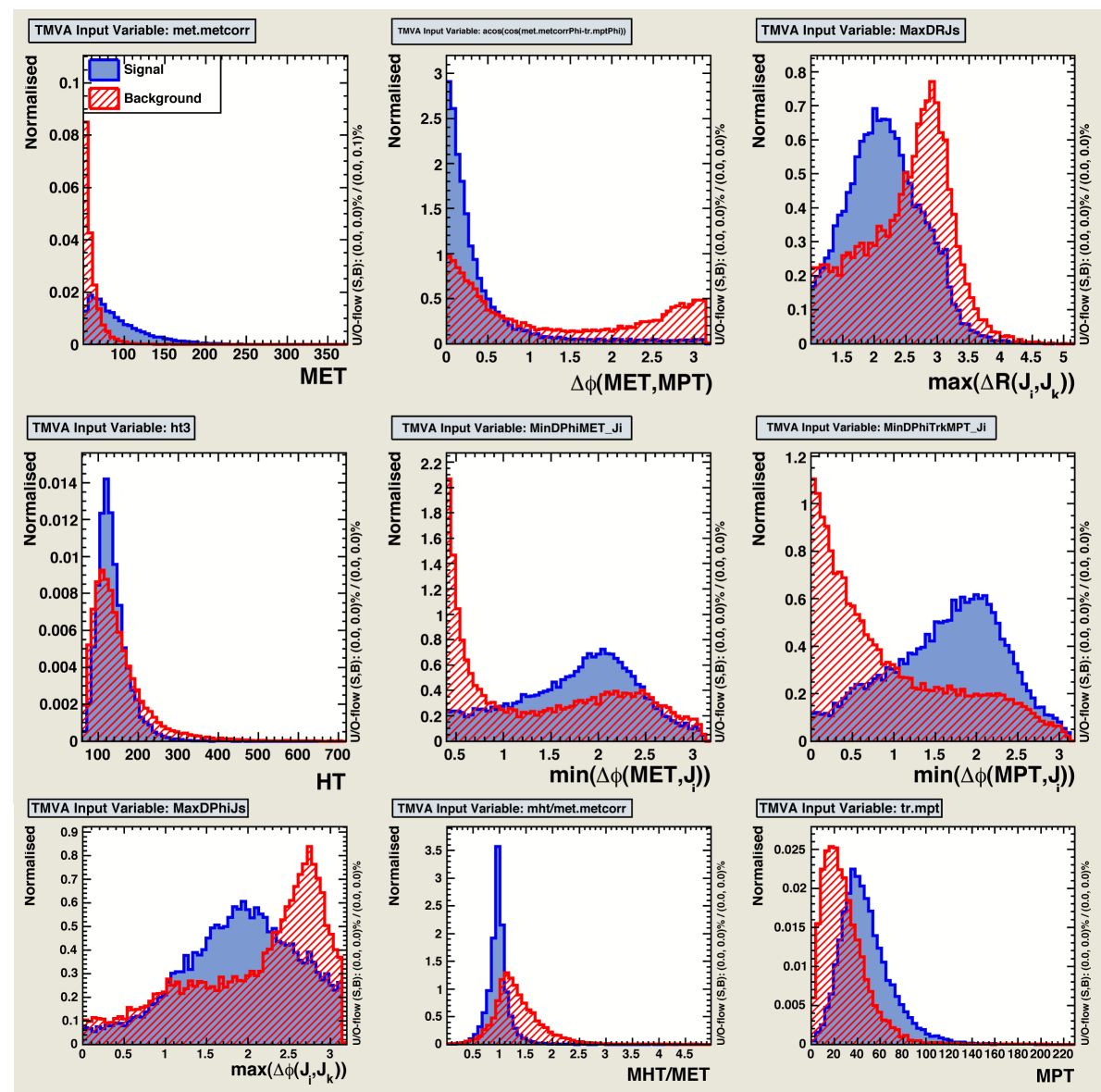

Figure 7.3.: Kinematic distributions of background and signal samples used in the $\mathrm{ANN}_{\mathrm{MJ}}$ training. The distributions are normalised to an arbitrary area. The signal is shown in solid histogram, and the QCD background in hatched.

distributions respectively. Since these probabilities are very close to one, we conclude that the ANN training has achieved generalization, and no overtraining has occurred.

Another important check is to see whether or not the ANN training has converged, i.e. if a stable minimum of the Eq. 7.3 was found. The result of this test is shown in Figure 7.4(b), where it can be seen that the "Estimator" $E$ has reached a minimum value, which is stable after $\sim 600$ epochs. After performing the above checks, we are confident that the $\mathrm{ANN}_{\mathrm{MJ}}$ training has achieved an optimal solution without overtraining. 


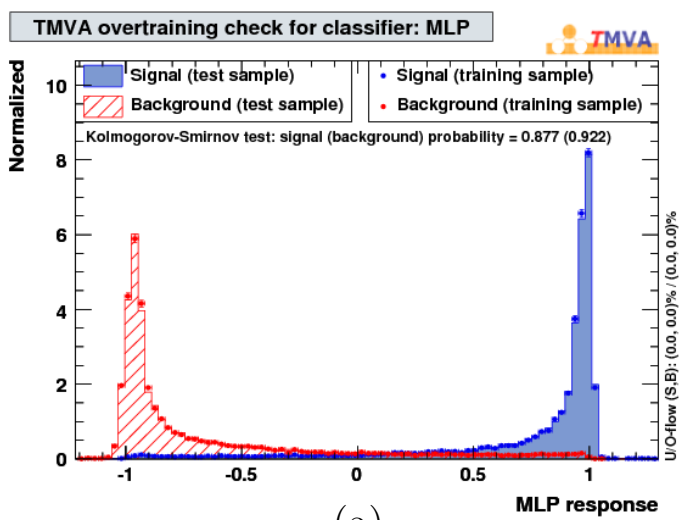

(a)

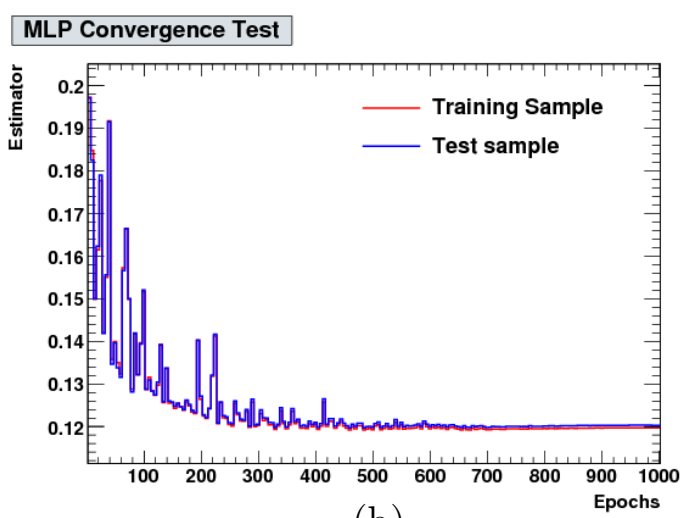

(b)

Figure 7.4.: Multijet rejection ANN performance: (a) $\mathrm{ANN}_{\mathrm{MJ}}$ output for the testing and training samples, (b) the convergence test of $\mathrm{ANN}_{\mathrm{MJ}}$.

As mentioned in the beginning of Section 7.2, we intend to increase the signal acceptance in this search, by employing the event selection based on the $\mathrm{ANN}_{\mathrm{MJ}}$ output, rather than using orthogonal cuts on kinematic variables. The following set of cuts was found to be optimal for high signal acceptance and background rejection, if we were to use cut-based event selection:

- $P_{T}^{t r}>20 \mathrm{GeV}$;

- $\notin_{T} / \sqrt{\mathrm{SumEt}}>4 \sqrt{G e V}$;

- $\Delta \phi\left(\not 中_{T}, \not P_{T}^{t r}\right)<1.6$.

where SumEt is the total transverse energy in the event. Events with real $\not_{T}$ have higher values of $\notin_{T} / \sqrt{\text { SumEt }}$ ratio.

Figure 7.5 shows the signal acceptance versus background rejection depending on a cut on the value of $\mathrm{ANN}_{\mathrm{MJ}}$. A comparison is made with the cut-based event selection, which is represented by " $\star$ " symbol in Figure 7.5. Selecting events with $A \mathrm{ANN}_{\mathrm{MJ}}>0$ achieves the same background rejection as in the cut-based selection, while increasing the signal acceptance by about 10\%. The performance of this cut regarding the QCD $\mathrm{MC}$ used for the training, the signal and the sum of all the backgrounds involved in the analysis is given in table 7.1. 


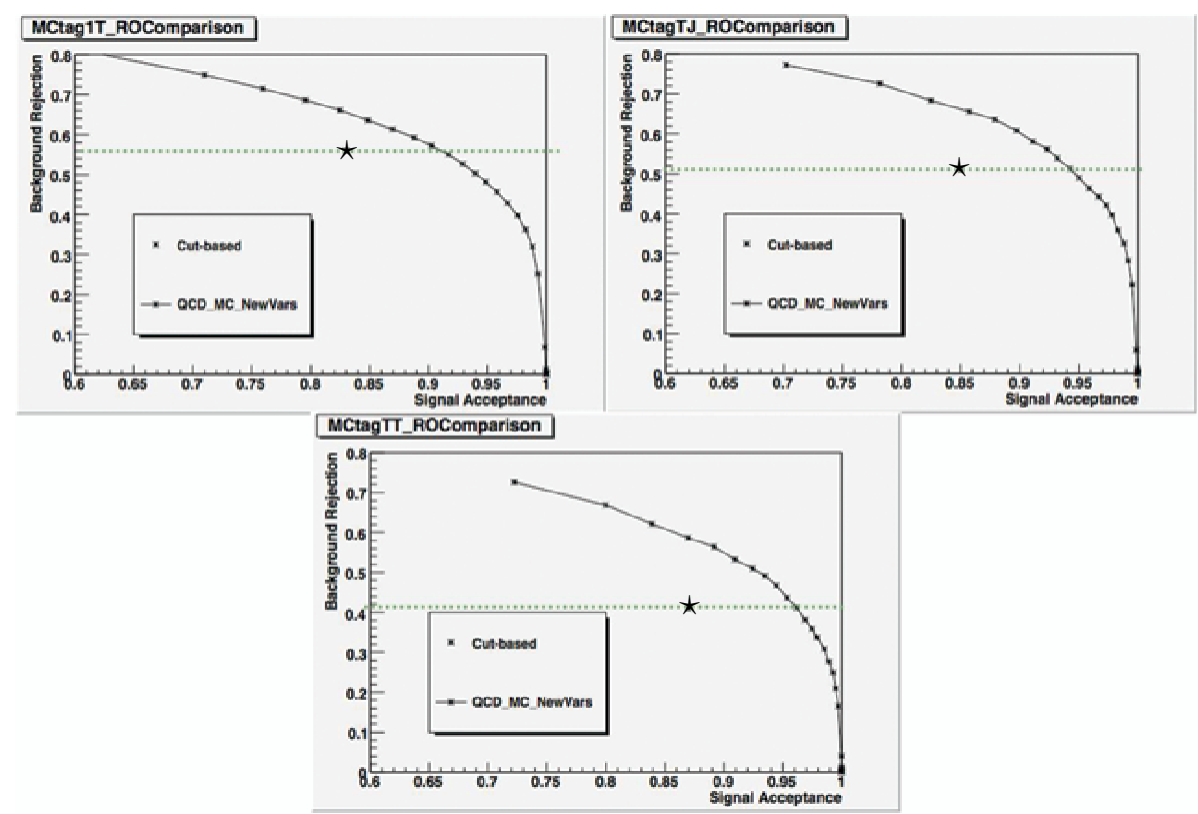

Figure 7.5.: Signal acceptance versus background rejection plot, scanning over values of $\mathrm{ANN}_{\mathrm{MJ}}$. The dotted line shows that using the $\mathrm{ANN}_{\mathrm{MJ}}$, we can keep the same background rejection as in the cut-based selection while increasing the signal acceptance by $\sim 10 \%$.

Since the $\mathrm{ANN}_{\mathrm{MJ}}$-based event selection clearly achieves a better signal significance ( see Appendix D), we define the events passing $\mathrm{ANN}_{\mathrm{MJ}}>0$ selection as the signal sample, which will be used in the search of the Higgs boson in these events.

Table 7.1: Rejection rates after $\mathrm{ANN}_{\mathrm{MJ}}>0$ selection for signal, all backgrounds and QCD MC only.

\begin{tabular}{|l|c|c|c|}
\hline & $Z H$ plus $W H$ & $\begin{array}{c}\text { Total } \\
\text { Background }\end{array}$ & QCD MC \\
\hline \hline Single Tag & $8.5 \%$ & $54.6 \%$ & $87.3 \%$ \\
ST+JP & $4.4 \%$ & $52.3 \%$ & $84.1 \%$ \\
ST+ST & $5.8 \%$ & $45.1 \%$ & $80.4 \%$ \\
\hline
\end{tabular}




\section{Summary of QCD Rejection ANN MJ $_{\text {Performance }}$}

The first step of our event selection is to reject most of our primary background: the QCD production of dijet/trijet events. The performance of the QCD MC-based $\mathrm{ANN}_{\mathrm{MJ}}$ is remarkable (Figure 7.4). A closer look at the $\mathrm{ANN}_{\mathrm{MJ}}$ output for the other background processes reveals the mistags are not completely removed: some of those events peak in the right side of $\mathrm{ANN}_{\mathrm{MJ}}$ output the distribution. This is in accord with our understanding of mistags coming from two different physics sources, QCD light flavor jets and $W / Z+$ light flavour events. We will focus on the latter and on other backgrounds in the following stages of the analysis.

The cut chosen, i.e. $\mathrm{ANN}_{\mathrm{MJ}}>0$, corresponds to keeping the same background rejection but enhancing signal acceptance with respect to a cut-based selection. It also enhances the signal significance of the three tagged subsamples by roughly $20 \%$. To summarize the performance of this neural network selection, we achieve an average signal rejection over the three tagging categories of $\sim 5 \%$, and an average background rejection of $\sim 50 \%$. As a result of this selection, the amount of QCD $b \bar{b} / c \bar{c}$ events is reduced by almost an order of magnitude. The signal events rejected by the neural network selection originate mostly from events where one of the jets is badly mismeasured. A summary of the event selection efficiencies at various stages of the analysis is shown in Tab. 7.2.

The choice of the input variables and the topology of the network was based upon the performance in discriminating the Higgs boson signal from the QCD background, where we use as a figure of merit the signal acceptance versus background rejection, as shown in Figure 7.5. The addition of more input variables and/or changes in the network size/topology did not provide any noticeable improvement. On the other hand, simplifying the network or removing input variables deteriorates the discrimination power of the network. The modeling of the $\mathrm{ANN}_{\mathrm{MJ}}$ is compared against data in control regions, as shown in Figure 7.7. 
The events in the region $\mathrm{ANN}_{\mathrm{MJ}}>0$ will constitute our final signal region, where we will perform a search for the Higgs boson signal.

Table 7.2: Acceptance of signal and backgrounds after selection criteria in $2.1 \mathrm{fb}^{-1}$. The events were weighted by cross-section and efficiencies after applying the selections.

\begin{tabular}{|l|c|c|c|c|c|}
\hline Selection cut & $\begin{array}{c}\text { \#Events } \\
\text { ZH(115) }\end{array}$ & Acc.(\%) & $\begin{array}{c}\text { \# Events } \\
\text { WH(115) }\end{array}$ & Acc.(\%) & Backgrounds \\
\hline \hline Good run & 33.1 & 100 & 91.3 & 100 & 14963805 \\
\hline Trigger driven selections & 8.05 & 24.2 & 17.0 & 18.62 & 523207 \\
Electron Veto & 8.0 & 23.9 & 13.5 & 14.8 & 512383 \\
Isolated Track Veto & 8.0 & 23.9 & 8.9 & 9.75 & 500813 \\
\hline E $_{T}$ not collinear to any jet & 7.6 & 23.0 & 7.9 & 8.7 & 56301 \\
\hline ANN MJ $>0_{\text {ST+ST }}$ & 7.2 & 21.7 & 6.7 & 7.3 & 24569 \\
\hline ST+JP & 1.00 & 3.1 & 0.86 & 1.0 & 105 \\
Exclusive ST & 0.81 & 2.6 & 0.74 & 0.8 & 148 \\
\hline
\end{tabular}



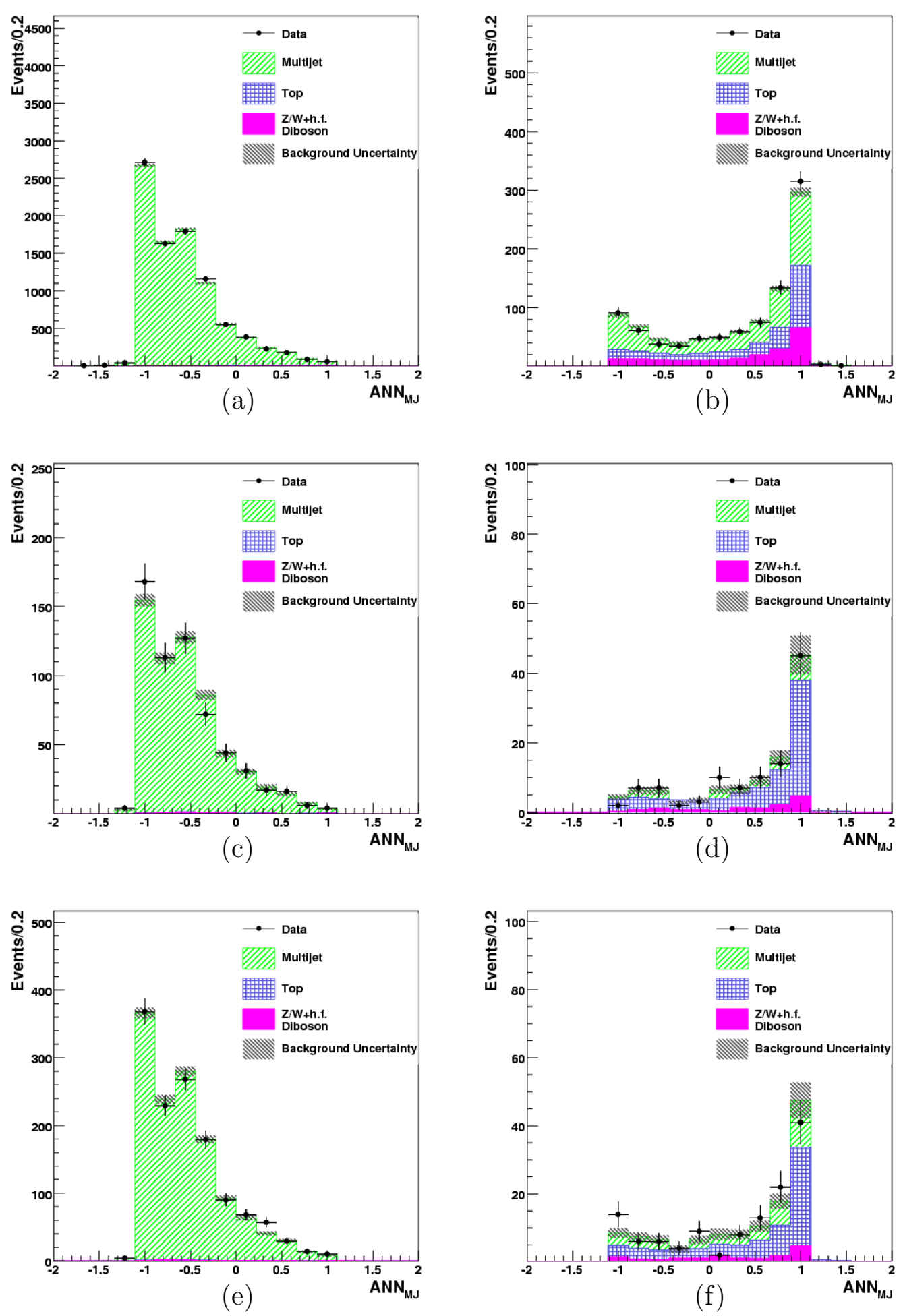

Figure 7.6.: The distribution of $\mathrm{ANN}_{\mathrm{MJ}}$ in control regions: Exclusive ST events in (a) CR1 and (b) CR2; ST+ST events in (c) CR1 and (d) CR2; ST+JP events in (e) CR1 and (f) CR2 


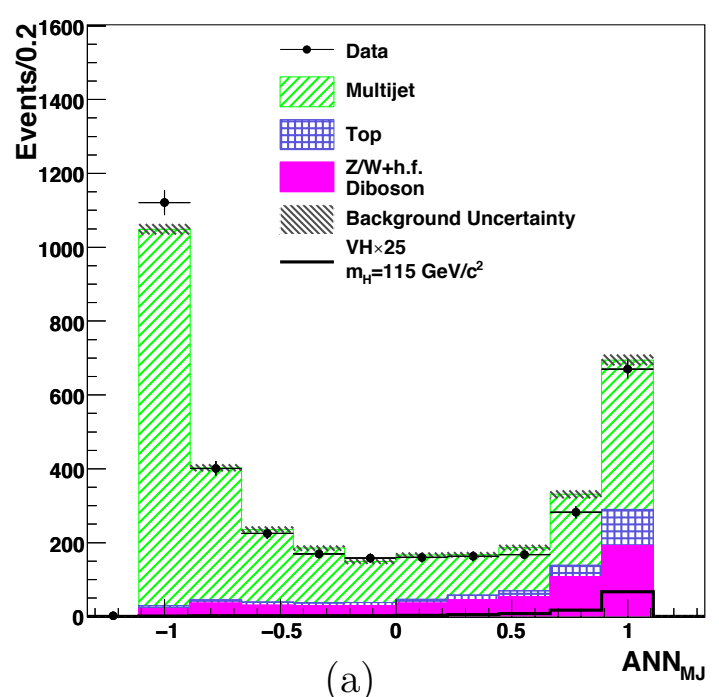

(a)

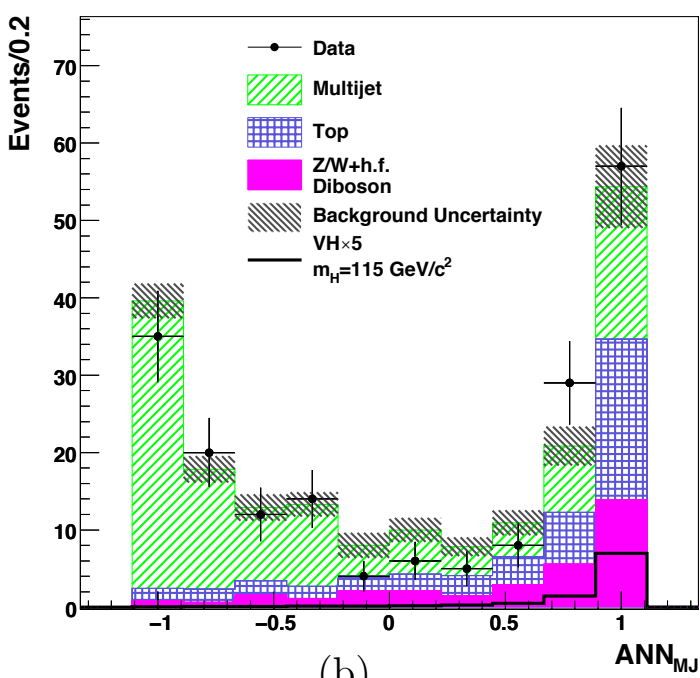

(b)

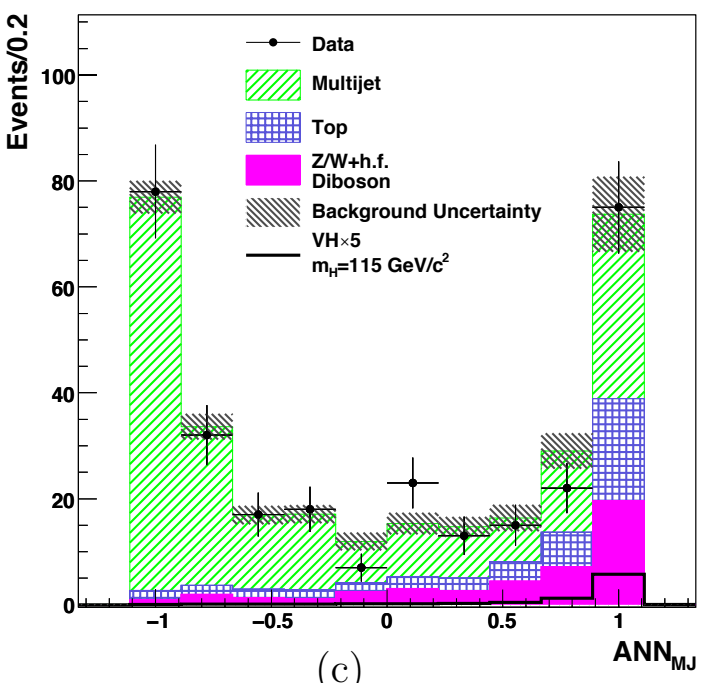

Figure 7.7.: The distribution of $\mathrm{ANN}_{\mathrm{MJ}}$ before applying the final selection of $\mathrm{ANN}_{\mathrm{MJ}}>0$ : events in (a) exclusive ET and (b) ST+ST and (c) ST+JP 


\subsection{QCD Enriched Control Region 3}

In order to test the data-driven estimation of QCD plus mistags in a more signallike region, we define an additional control region. This region aims at testing the multijet data-based modeling in a kinematic region which is very similar to the signal region. This region is defined by reversing the $\mathrm{ANN}_{\mathrm{MJ}}$ cut to remain blind to the signal region and is denoted as CR3 or control region 3.

- Control Region 3 (QCD dominated, signal like)

- All leptons are vetoed using the lepton identifications described in Section 3.8).

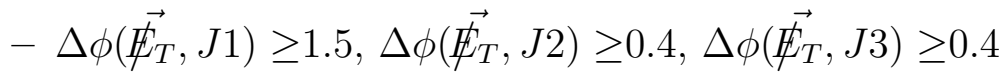

- $\mathrm{ANN}_{\mathrm{MJ}}<-0.5$ to have a high statistics sample where to check the data modeling as well as to extract the multijet normalization scale factor

The region with $-0.5<\mathrm{ANN}_{\mathrm{MJ}}<0$ is kept as a cross check of the multijet normalization (see Section 7.4).

A comparison of several distributions in CR3 is shown in Figures 7.8-7.10. It can be seen from this plots, that the CR3 is indeed dominated by multijet production, and our background model provides an adequate model of the data in this region. Table 7.3 summarizes the number of expected and observed events in CR3. 
Table 7.3: Number of expected and observed events in CR3. The expected Higgs boson signal is shown for $m_{H}=115 \mathrm{GeV} / c^{2}$. The uncertainties contain both Monte Carlo statistical error and systematic uncertainties.

\begin{tabular}{|l|c|c|c|}
\hline Process & Exclusive ST & ST+ST & ST+JP \\
\hline \hline QCD + Mistags & $1597.5 \pm 45.5$ & $60.0 \pm 7.2$ & $115.1 \pm 9.7$ \\
Single Top & $5.6 \pm 1.1$ & $0.8 \pm 0.2$ & $0.8 \pm 0.2$ \\
Top Pair & $17.1 \pm 2.5$ & $3.7 \pm 0.7$ & $3.7 \pm 0.7$ \\
Di-boson & $2.6 \pm 0.9$ & $0.2 \pm 0.1$ & $0.2 \pm 0.1$ \\
W + h.f. & $48.3 \pm 22.5$ & $0.8 \pm 0.6$ & $2.0 \pm 1.5$ \\
Z + h.f. & $23.9 \pm 12.0$ & $1.5 \pm 1.0$ & $1.2 \pm 0.8$ \\
\hline \hline Observed & 1695 & 67 & 123 \\
\hline \hline$Z H \rightarrow \nu \nu b b$ & 0.04 & 0.01 & 0.01 \\
\hline$W H \rightarrow(l) \nu b b$ & 0.13 & 0.03 & 0.03 \\
\hline$Z H \rightarrow(l l) b b$ & 0.02 & 0.01 & 0.01 \\
\hline
\end{tabular}



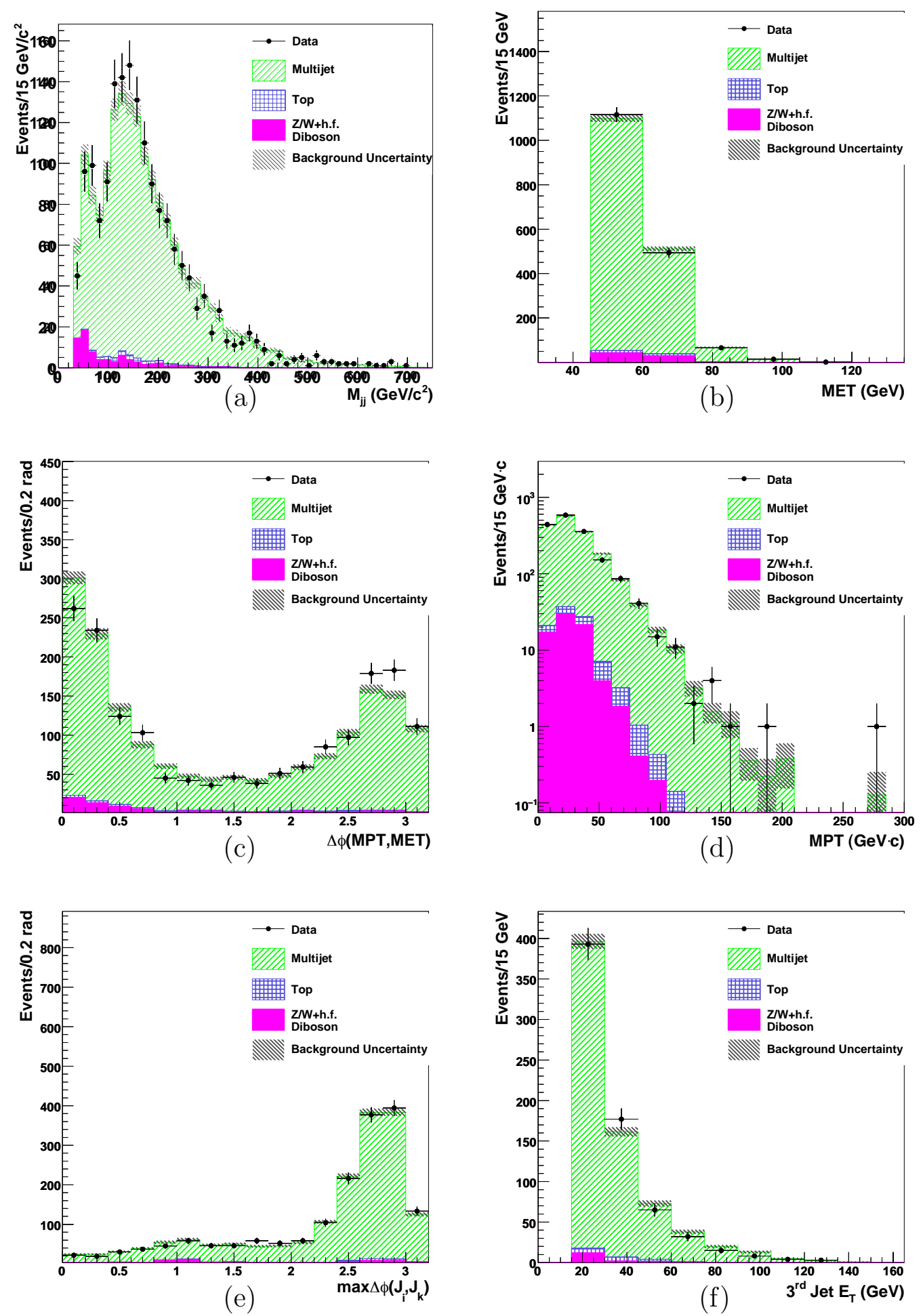

Figure 7.8.: Kinematic distributions in CR3 for events with single b-tag: (a) dijet invariant mass, (b) event $\not_{T}$, (c) $\Delta \phi\left(\overrightarrow{\not_{T}^{t r}}, \overrightarrow{\mathbb{E}_{T}}\right)$, (d) event $\not P_{T}^{t r}$, (e) maximum of the difference in $\phi$ between two jets directions, (f) transverse energy of the $3^{\text {rd }}$ jet 

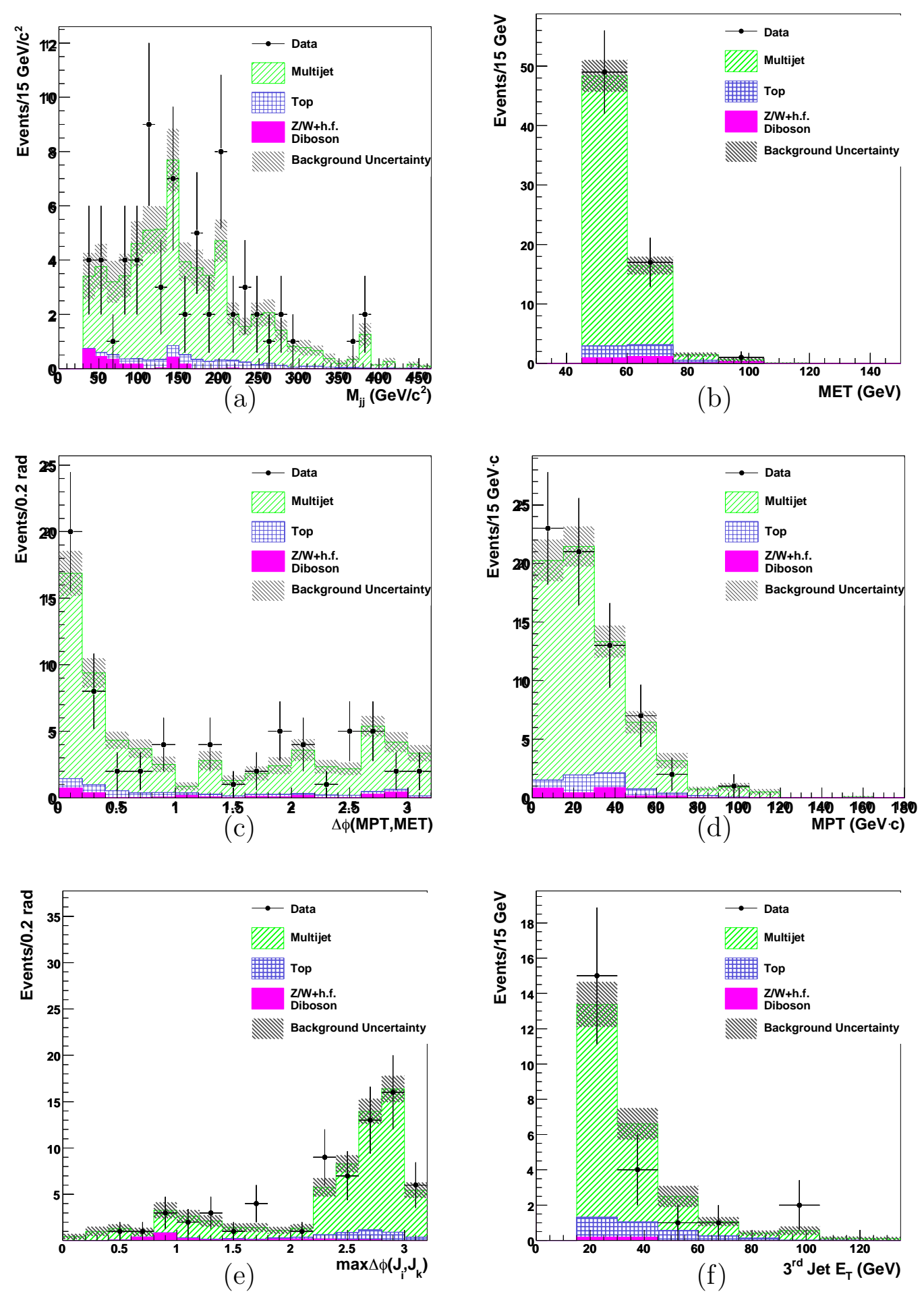

Figure 7.9.: Kinematic distributions in CR3 for ST+ST events: (a) dijet invariant mass, (b) event $\not_{T}$, (c) $\Delta \phi\left(\overrightarrow{\not_{T}^{t r}}, \overrightarrow{\mathscr{E}_{T}}\right)$, (d) event $\not_{T}^{t r}$, (e) maximum of the difference in $\phi$ between two jets directions, (f) transverse energy of the $3^{r d}$ jet 

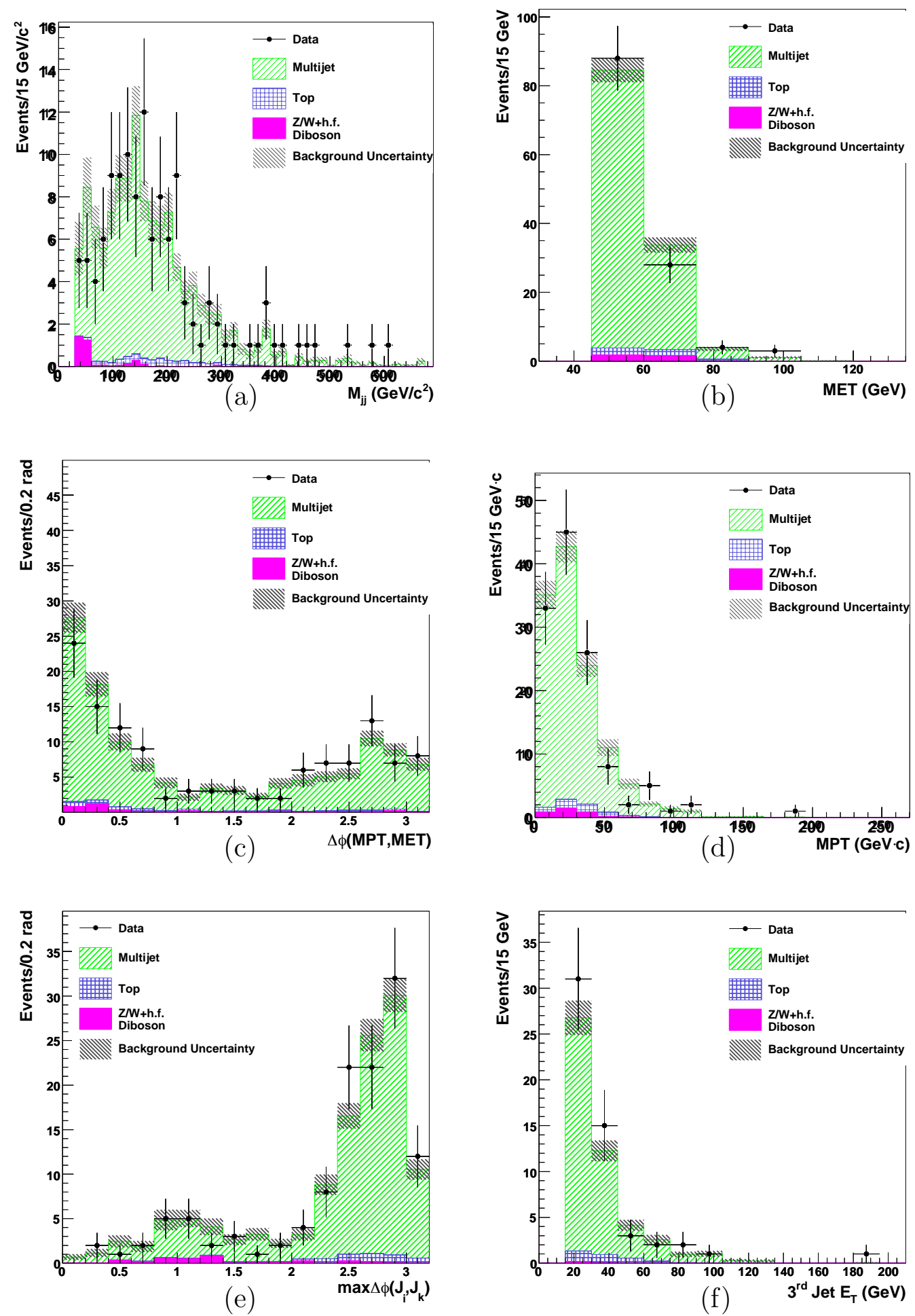

Figure 7.10.: Kinematic distributions in QCD CR1 for ST+JP events: (a) dijet invariant mass, (b) event $\not_{T}$, (c) $\Delta \phi\left(\overrightarrow{\not_{T}^{t r}}, \overrightarrow{\mathbb{E}_{T}}\right)$, (d) event $\not_{T}^{t r}$, (e) maximum of the difference in $\phi$ between two jets directions, (f) transverse energy of the $3^{\text {rd }}$ jet 


\subsection{Normalization of the Multijet Background}

In order to estimate the backgrounds originating from QCD heavy flavor multijet production as well as falsely tagged light flavor jet production, we use the Tag Rate Matrix (TRM) method described in Section 5.2.1. As noted above, this method describes well the shapes of the backgrounds. However, the normalization of the expected multijet background is not predicted well enough. In order to constrain the expected rates of these backgrounds we utilize CR2 and CR3, which are kinematic regions very close to the signal region. We first compute the normalization scale factor $k_{S F}$ in both of these regions. The uncertainties are derived from statistical uncertainties in the data and MC samples, and the systematic uncertainties on the MC samples. The uncertainties on the $k_{S F}^{a v e}$ will be used as the uncertainties on the multijet prediction in the signal region. We then compute the scale factor for multijet background to be used in signal region, $k_{S F}^{a v e}$, as the error weighted average of these two measurements.

Table 7.4: Multijet background scale factors in different control regions, for different tagging categories.

\begin{tabular}{|l|c|c|c|c|}
\hline Category & $k_{S F}$ in CR2 & $k_{S F}$ in CR3 & $k_{S F}^{\text {ave }}$ & $k_{S F}$ in $-0.5<\mathrm{ANN}_{\mathrm{MJ}}<0$ \\
\hline \hline Single Tag & $0.83 \pm 0.15$ & $1.13 \pm 0.06$ & $1.09 \pm 0.06$ & $1.07 \pm 0.15$ \\
ST+ST & $1.04 \pm 0.79$ & $0.97 \pm 0.21$ & $0.97 \pm 0.20$ & $0.64 \pm 0.33$ \\
ST+JP & $0.91 \pm 0.43$ & $0.76 \pm 0.12$ & $0.77 \pm 0.12$ & $0.65 \pm 0.25$ \\
\hline
\end{tabular}

After testing the multijet background performance in terms of reproducing the shapes of the observed distributions in CDF data in CR1 and CR2 and being confident that the shapes are well reproduced by the matrix, we extract the normalization factor as described above, and we use these values to normalize the multijet prediction in the signal region. The scale factors obtained in various control regions, as well as the scale factors we use in the signal region, $k_{S F}^{a v e}$, are listed in Table 7.4. As a final check 
before "opening the box", we check if the scale factors $k_{S F}^{a v e}$ provide a good model in the region $-0.5<\mathrm{ANN}_{\mathrm{MJ}}<0$. The last column in Table 7.4 shows the scale factors obtained in this region, and it can be seen that the results are consistent with each other within the systematic uncertainties.

\subsection{Signal Region Definition}

After thoroughly checking our background model, and demonstrating a good understanding of the sample composition, we also develop a new method to enhance the signal significance in the search. As described above, an ANN approach was chosen as an event selection tool, which achieves a big improvement over the cut-based selection. A normalization procedure for the remaining multijet background was developed. In the final signal region, all background normalizations will therefore be fixed, and the number of expected events in the signal region is fixed. In summary, the signal region is defined by the following set of selections, in addition to the definitions of the pretag sample given in Section 4.2.4:

- Signal Region

- All leptons are vetoed

$-\Delta \phi\left(\vec{\notin}_{T}, J 1\right) \geq 1.5, \Delta \phi\left(\vec{\notin}_{T}, J 2\right) \geq 0.4, \Delta \phi\left(\vec{\notin}_{T}, J 3\right) \geq 0.4$

$-\mathrm{ANN}_{\mathrm{MJ}}>0$ 


\section{Chapter 8. Search for the Higgs Boson}

As mentioned above, we selected a subset of the events in the MET+Jets sample using $\mathrm{ANN}_{\mathrm{MJ}}$, in order to maximize signal significance keeping a high signal efficiency. A large amount of signal events was accepted as a result of this event selection. As a final step in the analysis, we need to define the method to search for the presence of signal in the remaining dataset. In this chapter we describe our strategy in performing the search and present the results.

\subsection{Discriminant Neural Network}

To search for the evidence of a signal, the observed data is compared to the predictions of the model that includes signal and backgrounds ("signal hypothesis") and to the model that does not include signal ("null hypothesis") to see which model agrees with the observed data the best. There are various approaches that can be followed in this comparison. The most traditional one has been a simple counting experiment where one relies on a numerical excess of signal events over backgrounds. However, in searches for rare processes at hadron colliders, such an approach does not provide sufficient sensitivity due to very large backgrounds with large uncertainties, that often exceed the amount of the expected signal. A better sensitivity can be achieved by a fit to a kinematic distribution that distinguishes the events of signal process from backgrounds and comparing shapes and numbers of events in both signal and null hypotheses.

The resonance in the dijet invariant mass spectrum yields the most striking feature of the Higgs boson signal events, and searches at LEP and Tevatron have been performed by scanning this spectrum. Additional kinematic and topological features of the signal process can provide further discrimination from backgrounds, increasing 
the sensitivity of the search. These features can be combined into a single discriminating variable similar to the event selection ANN described in Section 7.2. The second $\mathrm{ANN}$, denoted as $\mathrm{ANN}_{\mathrm{SIG}}$, achieves a greater separation between signal and the remaining backgrounds. In this analysis, the output of the discriminant $\mathrm{ANN}_{\mathrm{SIG}}$ will be used to fit the distribution observed in data and scanned for the presence of the Higgs boson signal.

Since the fraction of expected multijet and $t \bar{t}$ events and some features of signal and backgrounds in 2- and 3- jet events are different, we perform the $\mathrm{ANN}_{\mathrm{SIG}}$ training for 2 and 3-jet events separately. We use for the $\mathrm{ANN}_{\mathrm{SIG}}$ training a background sample made of $75 \%$ of MET+Jets untagged data ${ }^{1}$ and $25 \%$ of $t \bar{t}$ for 2 -jet $\mathrm{ANN}_{\mathrm{SIG}}$ and $50 \%$ of MET+Jets untagged data and $50 \%$ of $t \bar{t}$ for 3-jet $\mathrm{ANN}_{\mathrm{SIG}}$. The Higgs signal used for the training is a mixture of $50 \% \mathrm{WH}$ events and $50 \% \mathrm{ZH}$ events. We optimized the $\mathrm{ANN}_{\mathrm{SIG}}$ training for various masses of the Higgs boson, in steps of $10 \mathrm{GeV} / c^{2}$. The topology of the $\mathrm{ANN}_{\mathrm{SIG}}$ consists of 6 input nodes with 1 bias node, 1 hidden layer with 12 hidden nodes, 1 hidden layer with 6 hidden nodes, and 1 output node, i.e. 7-12-6-1. The 6 input variables are:

- The invariant mass of the two leading jets: $M_{j j}$.

- The transverse invariant mass of all jets and $\mathbb{E}_{T}: M_{T, \nu+j e t s}$.

- The difference between scalar sum of all jets $E_{T}$ 's and $\not_{T}: H T-M E T$.

- The difference between vector sum of all jets $E_{T}$ 's and $\not_{T}: M H T-M E T$.

- The output of the TrackMET ANN: $A N N_{\text {trackMET. }}$

- The maximum of the difference in the $\mathrm{R}$ space between two jets (considering all pairings between jets in the event): $\max \left(\Delta R\left(J_{i}, J_{k}\right)\right)$.

The variable $A N N_{\text {trackMET }}$ is the output of an ANN developed at CDF [90] using tracking information to enhance the separation of events with real $\mathbb{E}_{T}$.

1 "untagged data" means the subset of the events in which all events with at least one SecVtx tag are removed 
The number of epochs for the $\mathrm{ANN}_{\mathrm{SIG}}$ training is set to 1000. The Figure 8.1 shows the distributions of the input variables for the training sample for 2-jet events and Figure 8.3 for 3 -jet events.
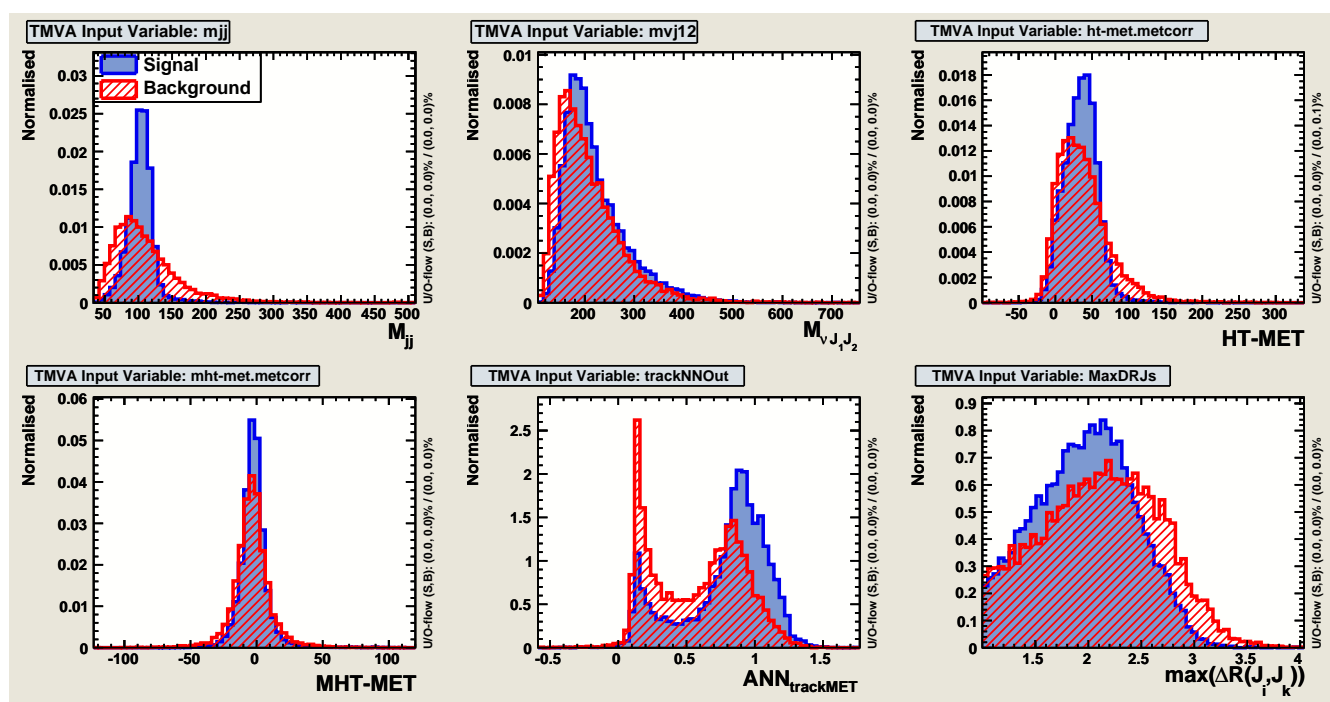

Figure 8.1.: Kinematic distributions of background and signal samples used in the training of $\mathrm{ANN}_{\mathrm{SIG}}$ for 2 jet events. The distributions are normalized to an arbitrary area. The signal is shown in solid histogram, and the sum of MET+Jets untagged data and $t \bar{t}$ background in hatched.

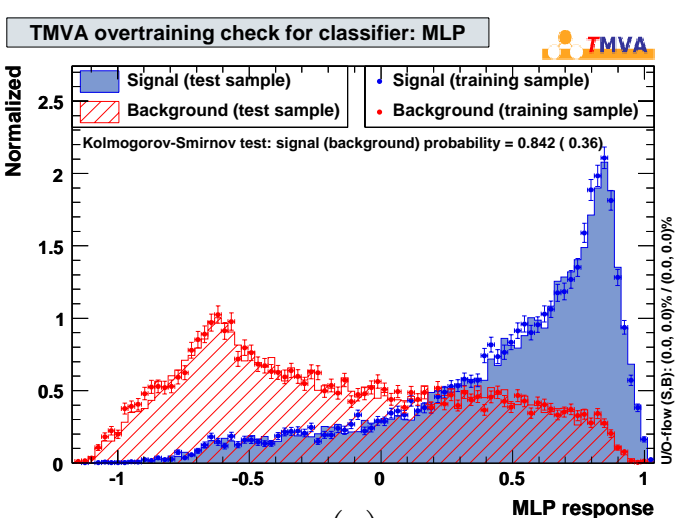

(a)

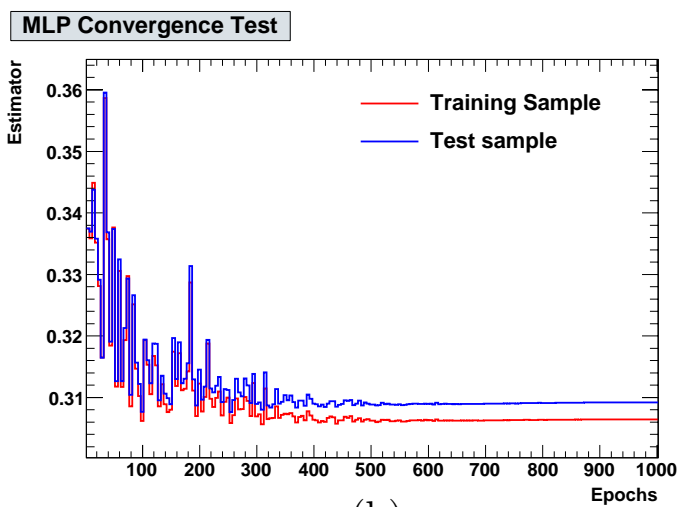

(b)

Figure 8.2.: Performance of $\mathrm{ANN}_{\mathrm{SIG}}$ for events with 2 jets: (a) $\mathrm{ANN}_{\mathrm{SIG}}$ output for the testing and training samples, (b) the convergence test of $\mathrm{ANN}_{\mathrm{SIG}}$. 

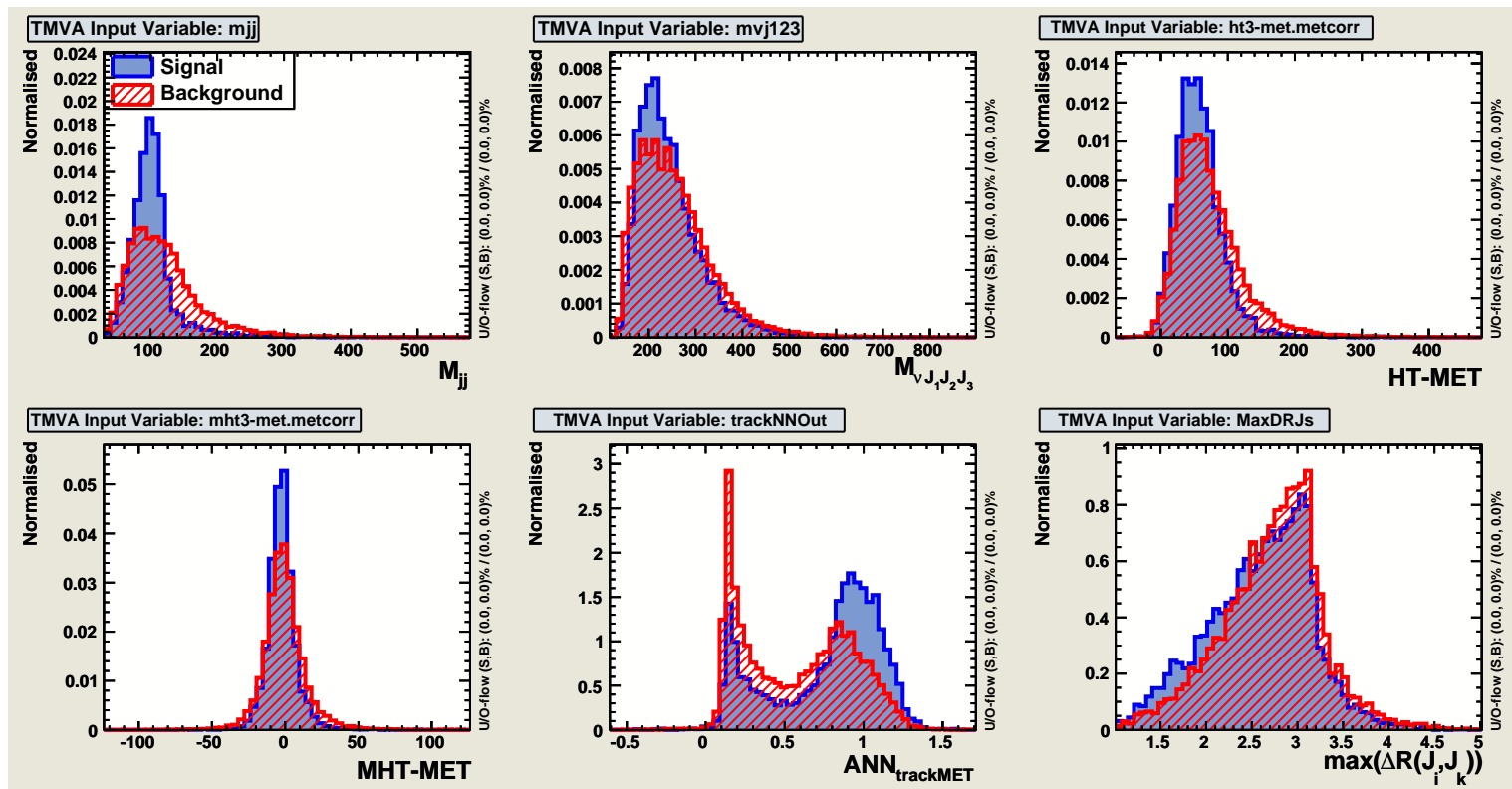

Figure 8.3.: Kinematic distributions of background and signal samples used in the training for 3 jet events. The distributions are normalised to an arbitrary area. The signal is shown in solid histogram, and the sum of MET+Jets untagged data and $t \bar{t}$ background in hatched.

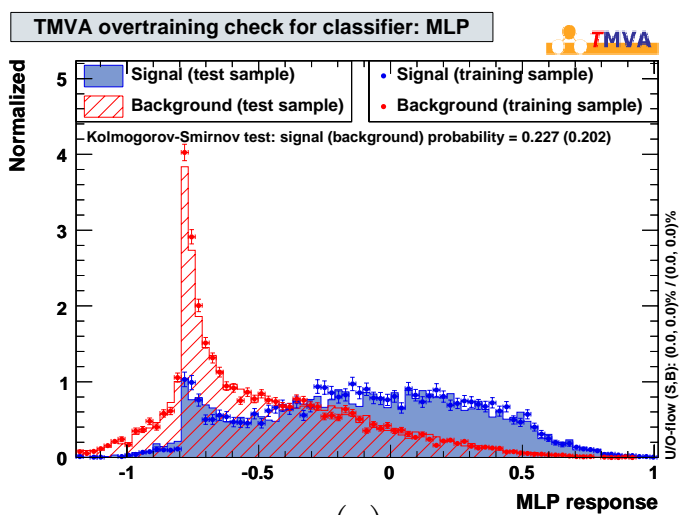

(a)

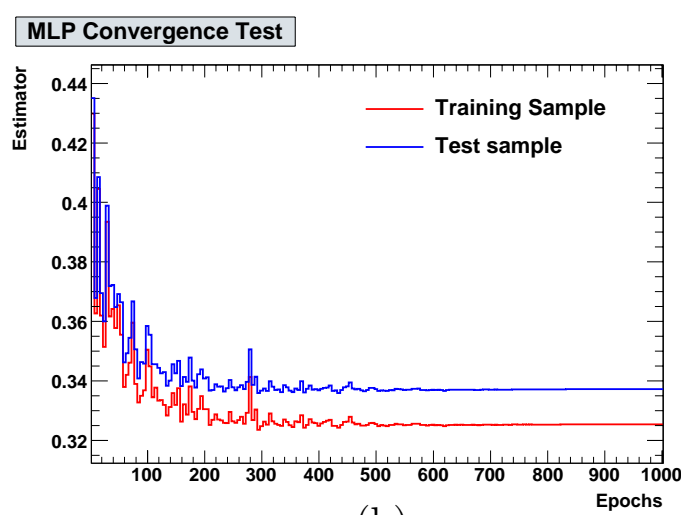

(b)

Figure 8.4.: Performance of $\mathrm{ANN}_{\mathrm{SIG}}$ for events with 3 jets: (a) $\mathrm{ANN}_{\mathrm{SIG}}$ output for the testing and training samples, (b) the convergence test of $\mathrm{ANN}_{\mathrm{SIG}}$. A spike of signal events observed in the background-like region of $\mathrm{ANN}_{\mathrm{SIG}}$ output $(-0.8,-0.6)$ is due to events where one of jets from the Higgs boson is detected as the third leading jet. This results in the wrong value of $M_{j j}$, which is constructed from the two leading jets. 


\subsection{Systematic Uncertainties}

Systematic uncertainties are uncertainties that can not be simply decreased by increasing the sample size. Systematic uncertainties arise from incomplete knowledge of effects like mechanical misalignment, electronic noise in detectors, variations in experimental conditions, mis-calibrations of detectors, etc. Systematic uncertainties may also originate from theoretical predictions when exact calculations are impossible, such as a limited knowledge about the parton distribution functions of the proton.

Systematic uncertainties result in the expectation value $E(X)$ of an observable $X$ being different from its true value $\lambda$, i.e.:

$$
b=E(X)-\lambda \neq 0
$$

A common procedure to estimate the systematic uncertainties is through a calibration of the measurement instruments (e.g. by calibrating the energies of charged leptons using a sample of $Z$ bosons) or by comparing with the Monte Carlo simulations. By estimating the precision of the calibration procedure one can try to estimate $b$. The measurement of the quantity $\lambda$ is then assigned a systematic uncertainty $b$, assuming that this uncertainty follows a Gaussian distribution around the mean value $E(X)$. According to this procedure, the systematic uncertainties can be treated as random errors, which is acceptable in the case of small systematic errors.

The systematic uncertainties considered in this analysis are classified as correlated (uncorrelated) depending on if they do (do not) affect both signal and the background processes. The summary of all uncertainties evaluated in this analysis are given in Tables 8.3-8.5.

- Luminosity Uncertainty. The procedure for determining the uncertainty on the luminosity collected in Run II is described in [52]. Since all Monte Carlo samples are normalized to the integrated luminosity of the analysis sample, this uncertainty affects all Monte Carlo samples. The total uncertainty on the luminosity measurement amounts to $6 \%$. 
- PDF uncertainty. The lack of precise knowledge of the parton distribution functions $(\mathrm{PDF})$ creates a source of a theoretical uncertainty for the amount of signal produced. We estimate this uncertainty by using different sets of PDF eigenvectors. The systematic uncertainty is computed by comparing the differences between CTEQ5L ${ }^{2}$ [91] and MRST72 [92] PDF parametrization sets, different $\Lambda_{Q C D}$ values in MRST72 and MRST75, and adding in quadrature the difference between CTEQ6M 20 pairs of eigenvectors. To calculate the total PDF uncertainty, we then add the larger of the 20 eigenvectors uncertainty or the MRST72 and CTEQ5L PDF sets uncertainty in quadrature with the $\Lambda_{Q C D}$ uncertainty. It has been found that a $2 \%$ uncertainty on the acceptance due to the choice of the PDF is sufficient.

- Jet Energy Scale Uncertainty. A systematic error in the calorimeter energy scale can affect the total transverse energy of the jets. The Jet Energy Scale (JES) uncertainty was determined by varying the jet energy correction factor by plus and minus one standard deviation while correcting the transverse energy of the jets [62]. This variation then propagates to the $\not_{T}$ reconstruction, the calculation of the azimuthal direction of $\mathbb{E}_{T}$, and thus to the expected number of events after applying the selection cuts. The influence of this variation is different for each background component; therefore, it needs to be determined separately by running the entire analysis code twice on all the simulated backgrounds. Since in some cases the JES can be asymmetric, we take the larger variation one as the estimation of JES uncertainty.

The variations in JES can also modify the shapes of various kinematic distributions that enter the $\mathrm{ANN}_{\mathrm{SIG}}$ calculation and affect its output. This variations are taken into account by assigning an uncertainty on shape of the $\mathrm{ANN}_{\mathrm{SIG}}$ distribution, by varying the JES by \pm one standard deviation for all background and signal Monte Carlo samples. Additionally, although the estimation of mul-

\footnotetext{
${ }^{2}$ which is the default PDF set used at CDF
} 
tijet background is derived from data, it involves a procedure of subtracting the Monte Carlo based backgrounds, which are affected by JES. Therefore, the variations from JES in the background estimations are propagated into shape variations of the multijet estimation.

Since in this analysis we use the $\mathrm{H} 1$ algorithm to correct jets, additional systematic uncertainties need to be assigned. This is done by varying $p_{T}$ of the tracks used in $\mathrm{H} 1$ algorithm by $\pm 3 \%$.

The total rate uncertainties due to JES variations are summarized in Tables 8.38.5 .

- Multijet background. The systematic error on the normalization is computed as explained in Section 7.4, yielding $5.5 \%$ in the single tagged channel, $20.6 \%$ in the $\mathrm{ST}+\mathrm{ST}$ and $15.6 \%$ in $\mathrm{ST}+\mathrm{JP}$.

Additionally, the variations in Tag Rate Matrix, which is used to estimate the multijet background, can also modify the shapes of distributions. It is taken into account by varying the tagging probability in each bin of the matrix by \pm one standard deviation, and by using the new shapes alternative shapes are used in the limit calculation.

The rest of the uncertainties listed in this section do not apply to the multijet predictions, since they are derived to describe the imperfections of the Monte Carlo simulations (such as JES or PDFs) or imperfect calculation of the integrated luminosity. Since the multijet background is derived directly from data, these sources of systematic uncertainties do apply.

- B-tagging scale factor. The efficiency of tagging a taggable jet in the simulation is different than in real events. This difference has to be taken into account when calculating the predicted number of events in the simulation after requiring a tag. For the Tight SecVtx tagger the scale factor is $0.95 \pm 0.01$ (stat) \pm 0.04 (syst), so we use a $4.1 \%$ error for all the simulated Monte Carlo components in the 
single and $8.2 \%$ in the ST+ST. For Jet Probability tagger $(<5 \%$ operating point) the data-MC scale factor is $0.846 \pm 0.017$ (stat) \pm 0.066 (syst), so we use 0.109\% uncertainty for ST+JP category.

- $W / Z+$ h.f. and diboson Monte Carlo next-to-leading order corrections. We use a $40 \%$ uncertainty on the $W$ and $Z$ background prediction and $11.5 \%$ on the diboson as described in $[79,80,81]$.

- Trigger Efficiency. The trigger efficiency study is described in [75]. Since we are using data below the region where the trigger is fully efficient, we assign systematic uncertainty to both MC based backgrounds and signal acceptances, by varying the trigger efficiency by $\pm 1 \sigma$. Table 8.1 summarizes the uncertainties assigned for each channel in all tagging categories.

Table 8.1: Uncertainties due to trigger efficiency

\begin{tabular}{|l|c|c|c|}
\hline Process & Exclusive ST & ST+ST & ST+JP \\
\hline \hline Single Top & $\pm 1.1 \%$ & $\pm 1.1 \%$ & $\pm 1.2 \%$ \\
Top Pair & $\pm 0.7 \%$ & $\pm 0.7 \%$ & $\pm 0.7 \%$ \\
Di-boson & $\pm 1.3 \%$ & $\pm 1.6 \%$ & $\pm 1.2 \%$ \\
W + h.f. & $\pm 2.0 \%$ & $\pm 1.7 \%$ & $\pm 1.8 \%$ \\
Z + h.f. & $\pm 1.4 \%$ & $\pm 1.3 \%$ & $\pm 1.3 \%$ \\
\hline \hline ZH & $\pm 0.9 \%$ & $\pm 1.0 \%$ & $\pm 1.2 \%$ \\
\hline \hline WH & $\pm 1.1 \%$ & $\pm 1.2 \%$ & $\pm 1.3 \%$ \\
\hline
\end{tabular}

- Lepton Veto. The uncertainty in the efficiency of the selection criteria used to select leptons were determined to be less than $2 \%$ [74].

- ISR/FSR. The uncertainty associated with the initial and final state radiation was evaluated for the signal samples. The variations to the amount of initial and final state radiations are studied by varying the $\Lambda_{Q C D}$ value used in the default CDF simulation. Additionally, we vary the squared transverse momentum evolution scale, $k_{T}^{2}$, which is used as a scale in $\alpha_{s}$ and PDFs when simulating 
the development of ISR showers. The variations in these variables result in the production of more or less ISR/FSR showers when compared to the default CDF simulation. The ISR/FSR uncertainties were evaluated for the ZH and WH signals separately when the Higgs mass was $115 \mathrm{GeV}$ by running the full analysis on these samples. As the total uncertainty we use the average variations weighted by the event yield in each channel considering the single and double tag separately (see Table 8.2)

Table 8.2: ISR/FSR uncertainties

\begin{tabular}{|c|c|c|c|c|c|c|}
\hline Process & \multicolumn{2}{|c|}{ Exclusive ST } & \multicolumn{2}{c|}{ ST+ST } & \multicolumn{2}{c|}{ ST+JP } \\
\hline \hline ZH $(120 \mathrm{GeV})$ & more & less & more & less & more & less \\
\hline ISR & $-1.0 \%$ & $-2.0 \%$ & $+0.01 \%$ & $+3.0 \%$ & $-1.0 \%$ & $+0.01 \%$ \\
FSR & $+2.0 \%$ & $-1.0 \%$ & $+3.00 \%$ & $+0.01 \%$ & $+1.0 \%$ & $+1.0 \%$ \\
\hline \hline WH $(120 \mathrm{GeV})$ & more & less & more & less & more & less \\
\hline ISR & $-1.0 \%$ & $-1.0 \%$ & $+8.0 \%$ & $+6.0 \%$ & $-5.0 \%$ & $+3.0 \%$ \\
FSR & $+1.0 \%$ & $+1.0 \%$ & $+6.0 \%$ & $+4.0 \%$ & $+2.0 \%$ & $+0.02 \%$ \\
\hline \hline Combined & more & less & more & less & more & less \\
\hline ISR & $-1.0 \%$ & $-1.53 \%$ & $+3.7 \%$ & $+4.4 \%$ & $-2.9 \%$ & $+1.41 \%$ \\
FSR & $+2.0 \%$ & $-0.10 \%$ & $+4.4 \%$ & $+1.8 \%$ & $+2.5 \%$ & $+0.5 \%$ \\
\hline
\end{tabular}


Table 8.3: Summary of systematic uncertainties in the Exclusive ST sample

\begin{tabular}{|c|c|c|c|c|c|c|c|c|}
\hline & $\mathrm{ZH}$ & WH & Multijet & $t \bar{t}$ & $\begin{array}{c}\text { single } \\
\text { top }\end{array}$ & Diboson & $W+$ h.f. & $Z+$ h.f. \\
\hline \multicolumn{9}{|c|}{ Correlated uncertainties } \\
\hline $\operatorname{Lumi}\left(\sigma_{\text {inel }}(p \bar{p})\right)$ & $4.0 \%$ & $4.0 \%$ & & $4.0 \%$ & $4.0 \%$ & $4.0 \%$ & $4.0 \%$ & $4.0 \%$ \\
\hline Lumi Monitor & $5.0 \%$ & $5.0 \%$ & & $5.0 \%$ & $5.0 \%$ & $5.0 \%$ & $5.0 \%$ & $5.0 \%$ \\
\hline Tagging SF & $4.1 \%$ & $4.1 \%$ & & $4.1 \%$ & $4.1 \%$ & $4.1 \%$ & $4.1 \%$ & $4.1 \%$ \\
\hline Trigger Eff. & $0.9 \%$ & $1.1 \%$ & & $0.7 \%$ & $1.1 \%$ & $1.3 \%$ & $2.0 \%$ & $1.4 \%$ \\
\hline Lepton Veto & $2.0 \%$ & $2.0 \%$ & & $2.0 \%$ & $2.0 \%$ & $2.0 \%$ & $2.0 \%$ & $2.0 \%$ \\
\hline $\mathrm{PDF}$ & $2.0 \%$ & $2.0 \%$ & & $2.0 \%$ & $2.0 \%$ & $2.0 \%$ & $2.0 \%$ & $2.0 \%$ \\
\hline JES & $\begin{array}{l}+3.8 \% \\
-3.8\end{array}$ & $\begin{array}{l}+3.8 \% \\
-3.8\end{array}$ & & $\begin{array}{l}+5.3 \% \\
-6.7\end{array}$ & ${ }_{-5.0}^{+4.8 \%}$ & $\begin{array}{l}+7.6 \% \\
-5.6\end{array}$ & ${ }_{-11.8}^{+13.0} \%$ & ${ }_{-8.9}^{+8.3 \%} \%$ \\
\hline ISR & & & & & & & & \\
\hline FSR & & & & & & & & \\
\hline \multicolumn{9}{|c|}{ Unorrelated uncertainties } \\
\hline $\begin{array}{l}\text { Cross-Section } \\
\text { Multijet Norm. }\end{array}$ & & & $5.5 \%$ & $6.2 \%$ & $15.9 \%$ & $11.5 \%$ & $40 \%$ & $40 \%$ \\
\hline
\end{tabular}

Table 8.4: Summary of systematic uncertainties in the ST+ST sample

\begin{tabular}{|c|c|c|c|c|c|c|c|c|}
\hline & $\mathrm{ZH}$ & WH & Multijet & $t \bar{t}$ & $\begin{array}{l}\text { single } \\
\text { top }\end{array}$ & Diboson & $W+$ h.f. & $Z+$ h.f \\
\hline \multicolumn{9}{|c|}{ Correlated uncertainties } \\
\hline $\operatorname{Lumi}\left(\sigma_{\text {inel }}(p \bar{p})\right)$ & $4.0 \%$ & $4.0 \%$ & & $4.0 \%$ & $4.0 \%$ & $4.0 \%$ & $4.0 \%$ & $4.0 \%$ \\
\hline Lumi Monitor & $5.0 \%$ & $5.0 \%$ & & $5.0 \%$ & $5.0 \%$ & $5.0 \%$ & $5.0 \%$ & $5.0 \%$ \\
\hline Tagging SF & $8.2 \%$ & $8.2 \%$ & & $8.2 \%$ & $8.2 \%$ & $8.2 \%$ & $8.2 \%$ & $8.2 \%$ \\
\hline Trigger Eff. & $1.0 \%$ & $1.2 \%$ & & $0.7 \%$ & $1.1 \%$ & $1.6 \%$ & $1.7 \%$ & $1.3 \%$ \\
\hline Lepton Veto & $2.0 \%$ & $2.0 \%$ & & $2.0 \%$ & $2.0 \%$ & $2.0 \%$ & $2.0 \%$ & $2.0 \%$ \\
\hline $\mathrm{PDF}$ & $2.0 \%$ & $2.0 \%$ & & $2.0 \%$ & $2.0 \%$ & $2.0 \%$ & $2.0 \%$ & $2.0 \%$ \\
\hline JES & ${ }_{-3.0}^{+3.0 \%}$ & ${ }_{-4.7}^{+3.5 \%}$ & & $\begin{array}{l}+5.0 \% \\
-6.7\end{array}$ & ${ }_{-4.5}^{+3.0 \%}$ & ${ }_{-7.3}^{+7.1 \%}$ & ${ }_{-11.9}^{+7.0} \%$ & $\begin{array}{l}+5.6 \% \\
-5.0\end{array}$ \\
\hline ISR & & & & & & & & \\
\hline FSR & & & & & & & & \\
\hline \multicolumn{9}{|c|}{ Unorrelated uncertainties } \\
\hline $\begin{array}{l}\text { Cross-Section } \\
\text { Multijet Norm. }\end{array}$ & & & $20.6 \%$ & $6.2 \%$ & $15.9 \%$ & $11.5 \%$ & $40 \%$ & $40 \%$ \\
\hline
\end{tabular}


Table 8.5: Summary of systematic uncertainties in the ST+JP sample

\begin{tabular}{|c|c|c|c|c|c|c|c|c|}
\hline & $\mathrm{ZH}$ & WH & Multijet & $t \bar{t}$ & $\begin{array}{c}\text { single } \\
\text { top }\end{array}$ & Diboson & $W+$ h.f. & $Z+$ h.f. \\
\hline \multicolumn{9}{|c|}{ Correlated uncertainties } \\
\hline$\overline{\operatorname{Lumi}}\left(\sigma_{\text {inel }}(p \bar{p})\right)$ & $4.0 \%$ & $4.0 \%$ & & $4.0 \%$ & $4.0 \%$ & $4.0 \%$ & $4.0 \%$ & $4.0 \%$ \\
\hline Lumi Monitor & $5.0 \%$ & $5.0 \%$ & & $5.0 \%$ & $5.0 \%$ & $5.0 \%$ & $5.0 \%$ & $5.0 \%$ \\
\hline Tagging SF & $10.9 \%$ & $10.9 \%$ & & $10.9 \%$ & $10.9 \%$ & $10.9 \%$ & $10.9 \%$ & $10.9 \%$ \\
\hline Trigger Eff. & $1.2 \%$ & $1.3 \%$ & & $0.7 \%$ & $1.2 \%$ & $1.2 \%$ & $1.8 \%$ & $1.3 \%$ \\
\hline Lepton Veto & $2.0 \%$ & $2.0 \%$ & & $2.0 \%$ & $2.0 \%$ & $2.0 \%$ & $2.0 \%$ & $2.0 \%$ \\
\hline $\mathrm{PDF}$ & $2.0 \%$ & $2.0 \%$ & & $2.0 \%$ & $2.0 \%$ & $2.0 \%$ & $2.0 \%$ & $2.0 \%$ \\
\hline JES & ${ }_{-3.7}^{+3.7 \%}$ & ${ }_{-4.0}^{+4.0 \%}$ & & ${ }_{-6.2}^{+5.5 \%}$ & ${ }_{-4.6}^{+4.4 \%}$ & $\begin{array}{l}+6.7 \% \\
-6.9\end{array}$ & ${ }_{-6.4}^{+8.5 \%}$ & ${ }_{-6.8}^{+4.8 \%}$ \\
\hline ISR & & & & & & & & \\
\hline FSR & & & & & & & & \\
\hline \multicolumn{9}{|c|}{ Unorrelated uncertainties } \\
\hline $\begin{array}{l}\text { Cross-Section } \\
\text { Multijet Norm. }\end{array}$ & & & $15.6 \%$ & $6.2 \%$ & $15.9 \%$ & $11.5 \%$ & $40 \%$ & $40 \%$ \\
\hline
\end{tabular}




\subsection{Measurement in the Signal Region}

As it was shown in the previous chapters, we have achieved a good understanding of the MET+Jets sample in the control regions. Due to a broader class of events analyzed in this analysis, such as events with three jets and a new ANN approach in event selection, the signal acceptance has dramatically increased with respect to the previous searches in this channel $[74,90]$. To further increase the expected significance of the signal and to obtain a more stringent limit, we developed a discriminant $\mathrm{ANN}_{S I G}$ to separate events from Higgs boson production and the backgrounds. A summary of systematic uncertainties evaluated for this search is given in the Section 8.2.

At this point, the signal box is opened to perform the search for the Higgs boson. The expected and observed event yields in signal region are listed in Table 8.6. The uncertainties on the total number of expected events are calculated by taking into account the correlations between some of the systematic uncertainties. The comparisons of kinematic variables in the signal region are shown in Figures 8.5-8.7. The $\mathrm{ANN}_{\mathrm{SIG}}$ output, which is used to scan for the presence of a signal, is shown in Figure 8.8. 
Table 8.6: Comparison of the total number of expected and observed events in the signal region for different $b$-tagging categories. The uncertainties contain both statistical and systematic uncertainties. The expected signal is shown for $m_{H}=115 \mathrm{GeV} / c^{2}$.

\begin{tabular}{|l|c|c|c|}
\hline Process & Exclusive ST & ST+ST & ST+JP \\
\hline \hline QCD + Mistags & $941.2 \pm 86.0$ & $42.1 \pm 12.4$ & $78.2 \pm 17.0$ \\
Single Top & $43.2 \pm 7.9$ & $8.5 \pm 1.7$ & $7.2 \pm 1.5$ \\
Top Pair & $124.5 \pm 17$ & $27.4 \pm 4.3$ & $27.1 \pm 4.6$ \\
Di-boson & $35.6 \pm 6.8$ & $4.9 \pm 1.2$ & $4.3 \pm 1.1$ \\
W + h.f. & $296.9 \pm 129.5$ & $11.0 \pm 6.5$ & $21.0 \pm 10.6$ \\
Z + h.f. & $107.0 \pm 45.8$ & $10.8 \pm 5.0$ & $11.3 \pm 5.2$ \\
\hline \hline Total Exp & $1548.4 \pm 168.1$ & $104.6 \pm 16.0$ & $149.1 \pm 22.0$ \\
\hline \hline Observed & 1443 & 105 & 148 \\
\hline \hline$Z H \rightarrow \nu \nu b b$ & 2.1 & 1.0 & 0.8 \\
\hline$W H \rightarrow(l) \nu b b$ & 1.8 & 0.9 & 0.7 \\
\hline$Z H \rightarrow(l l) b b$ & 0.09 & 0.04 & 0.03 \\
\hline
\end{tabular}



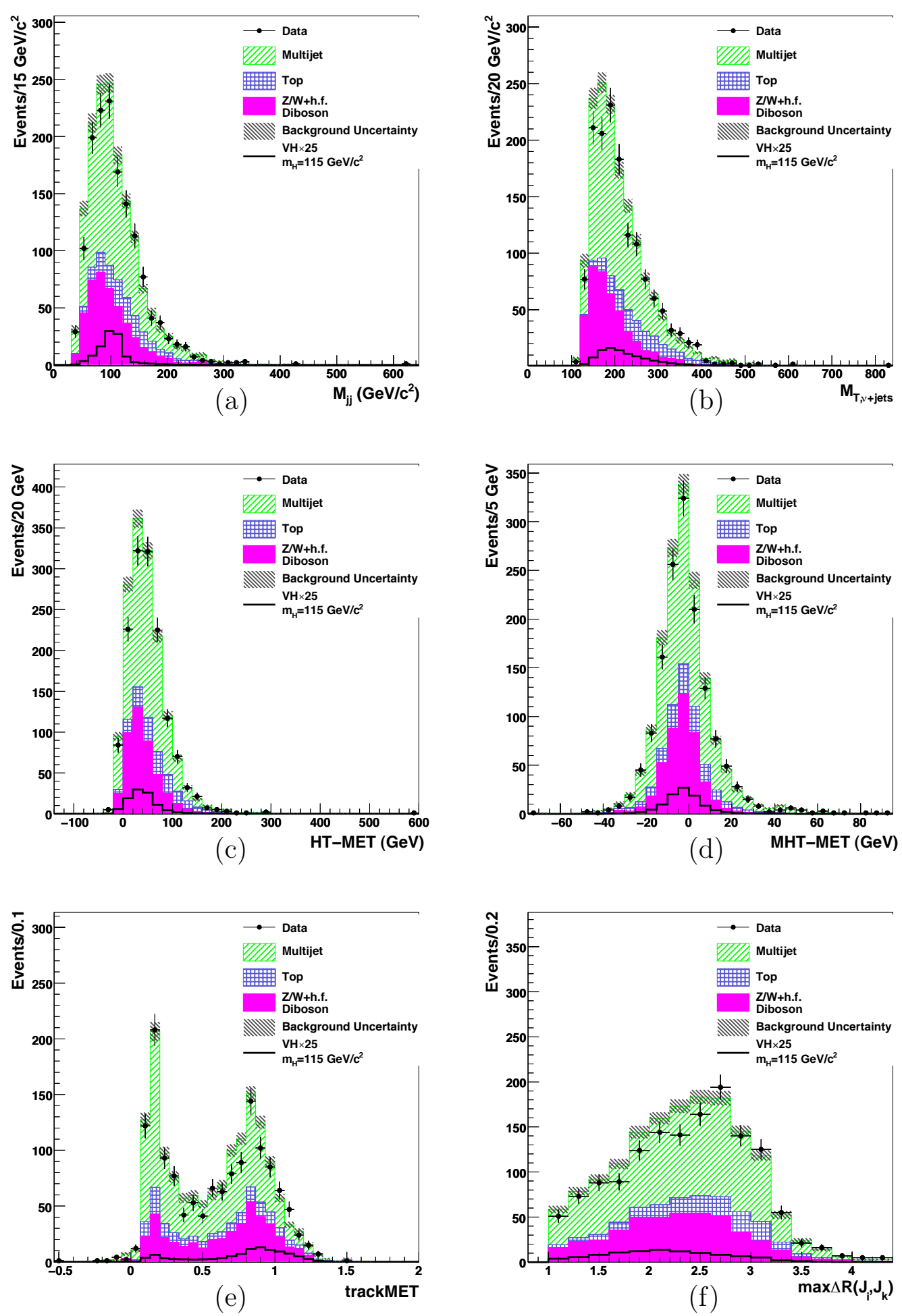

Figure 8.5.: Kinematic distributions of the inputs to the $\mathrm{ANN}_{\mathrm{SIG}}$ in the signal region for Exclusive ST events: (a) dijet invariant mass, (b) invariant mass of all jets and $\mathbb{E}_{T}$, (c) event $H_{T}-\not_{T}$, (d) event $\not H_{T}-\not_{T}$, (e) $\mathrm{ANN}_{\text {trackMET }}$, (f) maximum $\Delta R$ between all jets. The distributions for the $m_{H}=115 \mathrm{GeV} / c^{2}$ Higgs boson signal are shown in the overlaid black histogram (scaled by a factor of 25 ). 

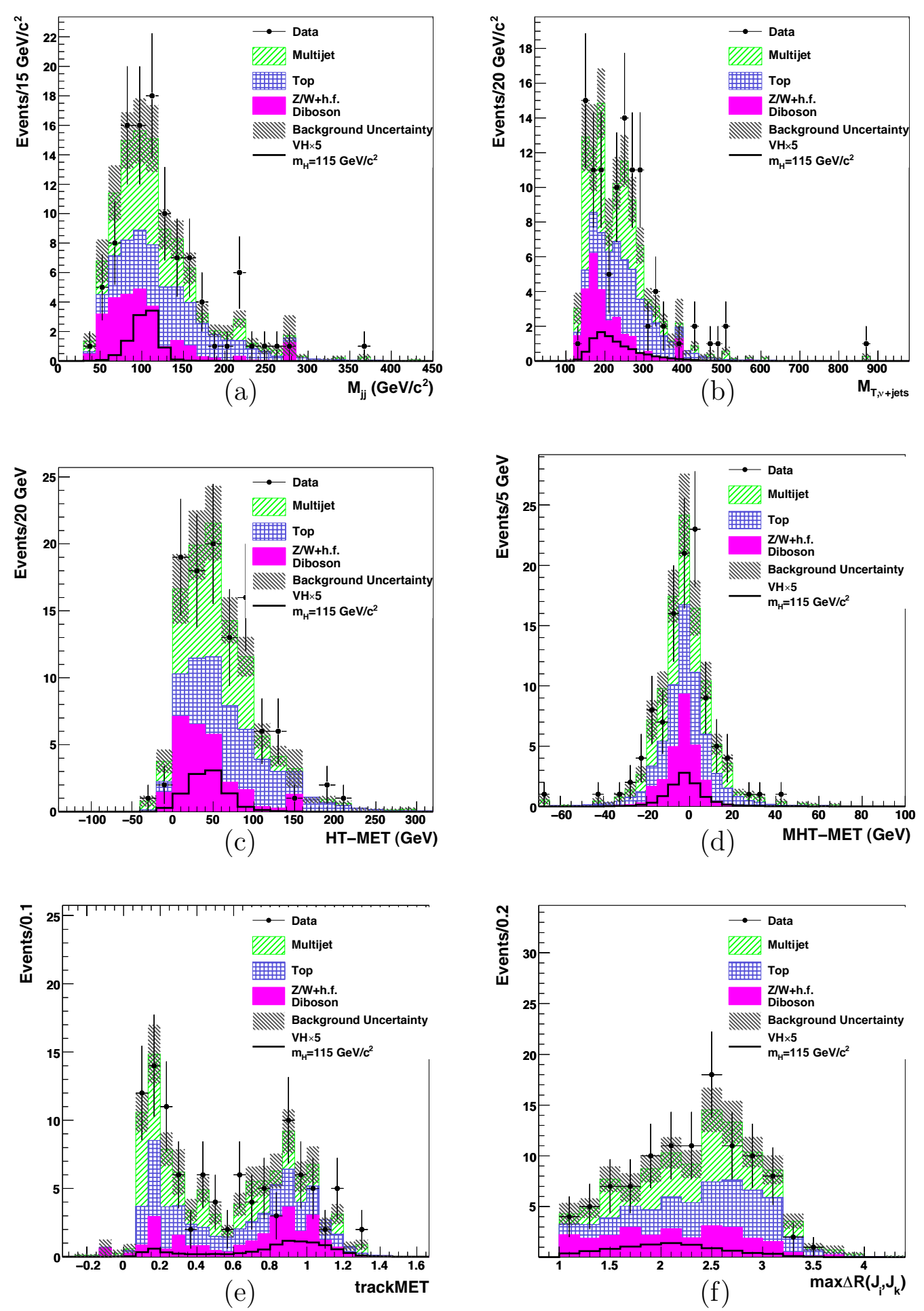

Figure 8.6.: Kinematic distributions of the inputs to the $\mathrm{ANN}_{\mathrm{SIG}}$ in the signal region for ST+ST events: (a) dijet invariant mass, (b) invariant mass of all jets and $\mathbb{E}_{T}$, (c) event $H_{T}-\mathbb{E}_{T}$, (d) event $\not H_{T}-\mathbb{E}_{T}$, (e) $\mathrm{ANN}_{\text {trackMET }}$, (f) maximum $\Delta R$ between all jets. The distributions for the $m_{H}=115 \mathrm{GeV} / c^{2}$ Higgs boson signal are shown in the overlaid black histogram (scaled by a factor of 5 ). 

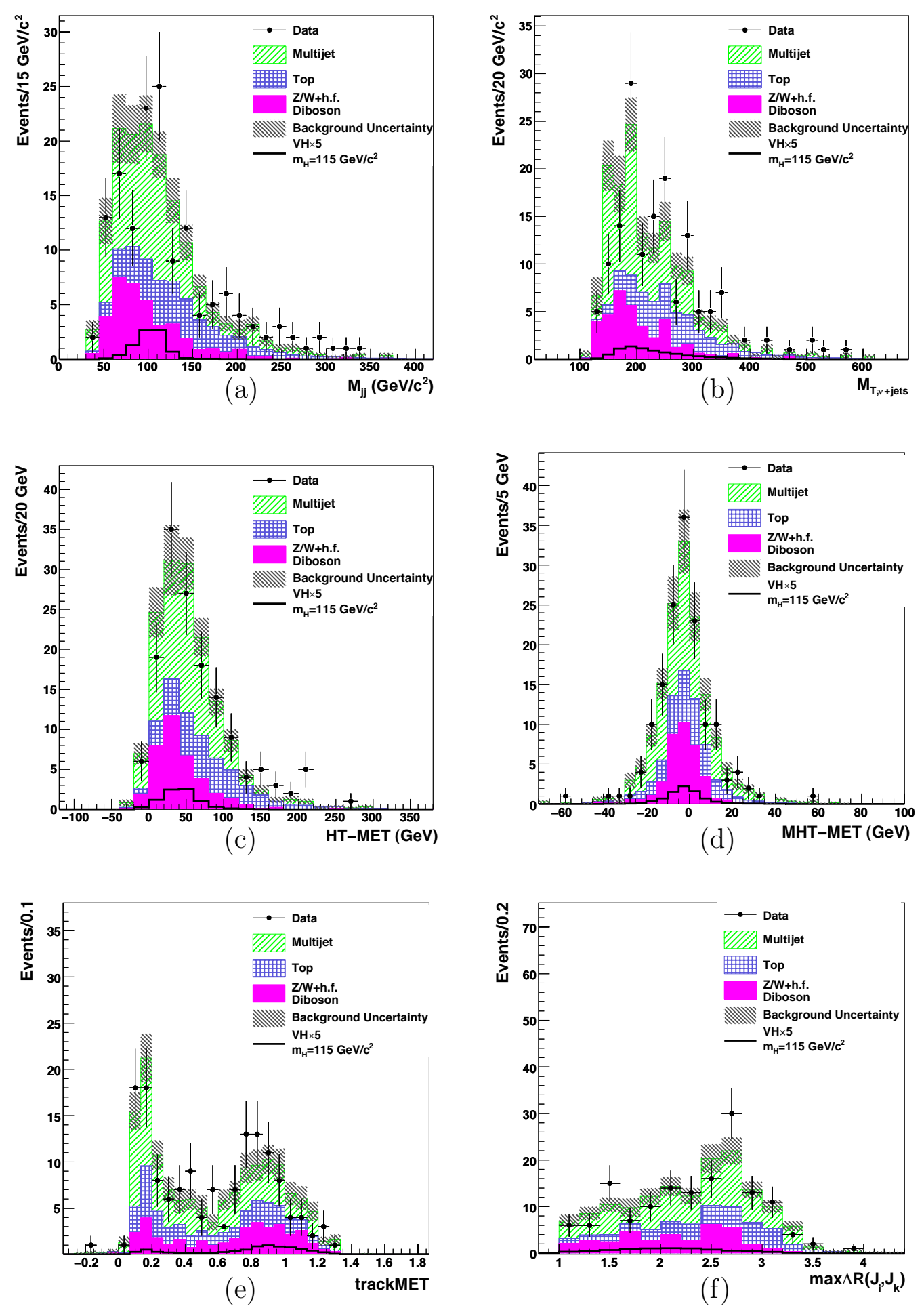

Figure 8.7.: Kinematic distributions of the inputs to the $\mathrm{ANN}_{\mathrm{SIG}}$ in the signal region for ST+JP events: (a) dijet invariant mass, (b) invariant mass of all jets and $\mathbb{E}_{T}$, (c) event $H_{T}-\mathbb{E}_{T}$, (d) event $\not H_{T}-\mathbb{E}_{T}$, (e) $\mathrm{ANN}_{\text {trackMET }}$, (f) maximum $\Delta R$ between all jets. The distributions for the $m_{H}=115 \mathrm{GeV} / c^{2}$ Higgs boson signal are shown in the overlaid black histogram (scaled by a factor of 5 ). 


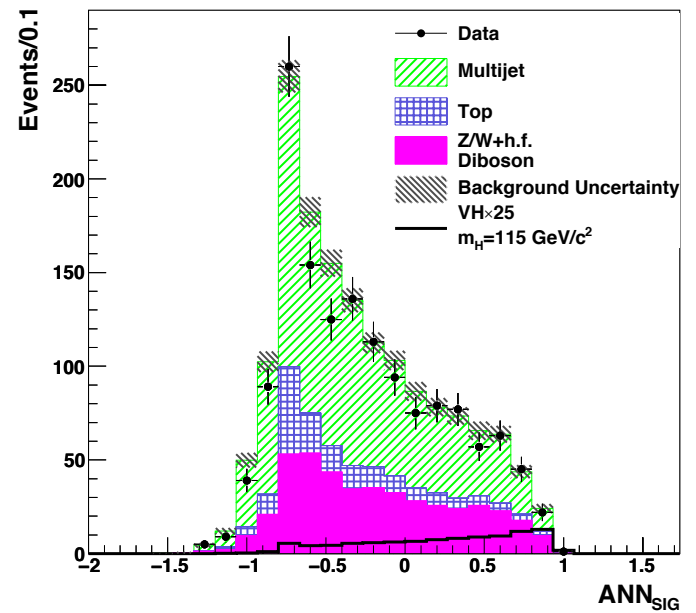

(a) $\mathrm{ANN}_{\mathrm{SIG}}$ output in Signal Region, Exclusive Single Tag

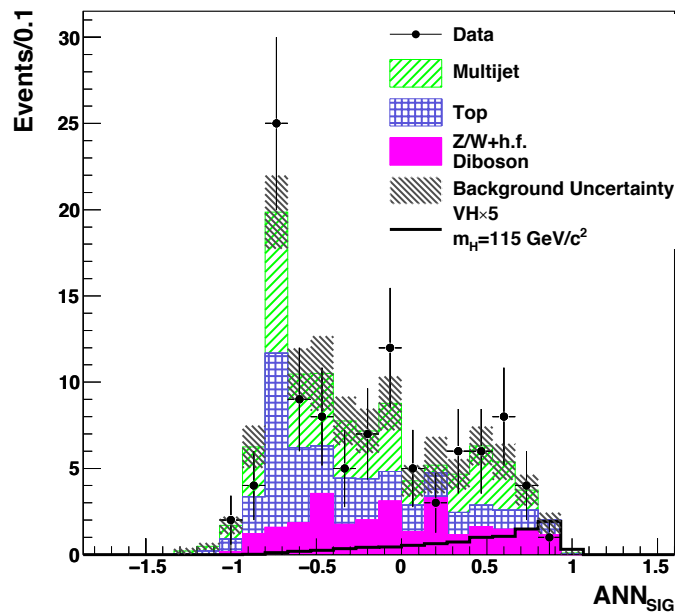

(b) $\mathrm{ANN}_{\mathrm{SIG}}$ output in Signal Region, $\mathrm{ST}+\mathrm{ST}$

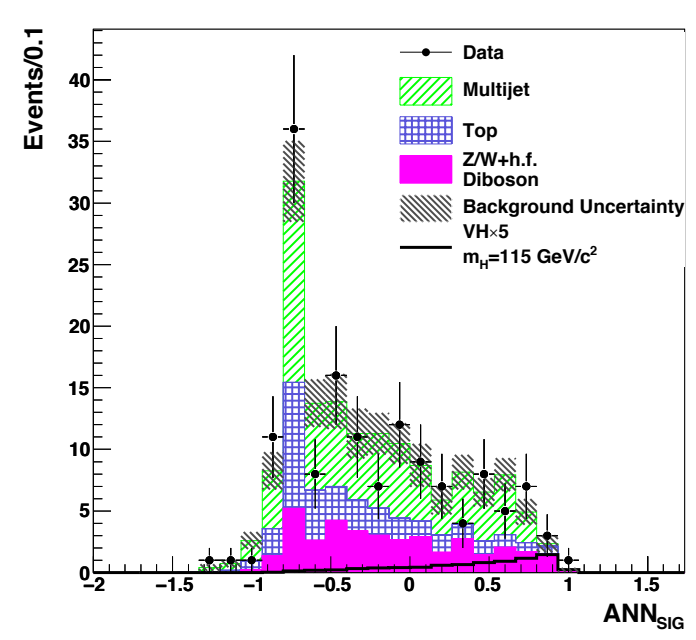

(c) $\mathrm{ANN}_{\mathrm{SIG}}$ output in Signal Region, $\mathrm{ST}+\mathrm{JP}$

Figure 8.8.: $\mathrm{ANN}_{\mathrm{SIG}}$ distributions in the signal region. The distributions for the $m_{H}=115 \mathrm{GeV} / c^{2}$ Higgs boson signal are shown in an overlaid black histogram (scaled by a factor of 25 for single-tagged events and a factor of 5 for double-tagged events). 


\subsection{Limit Calculation}

As shown in Section 8.3, the observed event kinematics and $\mathrm{ANN}_{\mathrm{SIG}}$ show no significant excess above SM predictions. Since the data agrees with background estimation, there is no indication of a signal of the Higgs boson production. Therefore, we place an upper limit on the cross-section of the Higgs boson production in association with a $W$ or $Z$ boson. In order to calculate the upper limits, we analyze the binned $\mathrm{ANN}_{\mathrm{SIG}}$ discriminant distribution, incorporating all systematic uncertainties described above, to test for a $Z H$ or $W H$ signal in the presence of SM backgrounds.

The limits are computed using the Bayesian likelihood method [93,94] with flat prior probability for the signal cross section and Gaussian priors for the uncertainties on acceptance and backgrounds. The software package we use for limit computation is called MCLIMIT [95], and contains an implementation of a multi-channel Bayesian limit calculator [96], that allows to consider the correlations between various acceptance and background priors.

In the simplest case of a counting experiment and a single source of background, Bayesian approach to calculate the limit is the following. Let us assume an experiment is conducted, and $n$ events (Poisson distributed) are observed, with a mean expectation $s \epsilon+b$, where $s$ is the number of expected signal events, $\epsilon$ is the acceptance (product of branching fraction, detector efficiency, luminosity factor, etc.) and $b$ is the number of expected background events. Let us assume that the background is known precisely, and the signal acceptance is known with a precision $\sigma_{\epsilon}$ from a subsidiary measurements. The Bayesian theorem then states that the posterior $p(s, \epsilon \mid n)$ for $s$ is:

$$
p(s, \epsilon \mid n)=\frac{P(n \mid s, \epsilon) \pi(s) \pi(\epsilon)}{\iint P(n \mid s, \epsilon) \pi(s) \pi(\epsilon) d s d \epsilon}
$$

where $P(n \mid s, \epsilon)$ is the probability of observing $n$ events given a signal $s$ with acceptance $\epsilon$. The functions $\pi(s)$ and $\pi(\epsilon)$ are the prior probability densities for $s$ and $\epsilon$ respectively. Since there is no experimental information about the Higgs boson, in 
the Bayesian technique a flat prior is assigned to $s$ (i.e. $\pi(s)=1$ ). The number of observed events in data follows Poisson distribution, therefore:

$$
P(n \mid s, \epsilon)=\frac{(s \epsilon+b)^{n}}{n !} e^{-(s \epsilon+b)}
$$

We can obtain the posterior for $s$ by "marginalizing" over $\epsilon$ :

$$
p(s \mid n)=\int_{0}^{\infty} p(s, \epsilon) d \epsilon
$$

Finally, to obtain the limit on $s$, the posterior density function $p(s \mid n)$ is integrated until the desired confidence level (C.L.), i.e. by solving the following equation for $s_{u}$ :

$$
\int_{0}^{s_{u}} p(s \mid n) d s=0.95
$$

an upper limit for $s_{u}$ at $95 \%$ C.L. can be found.

In order to increase the sensitivity of this analysis, we use three independent samples, split by tagging categories, which we combine at the end. As described in the Section 8.1, we scan the distributions of the $\mathrm{ANN}_{\mathrm{SI}}$ in the search for a signal excess, using a binned distribution, i.e. a histogram. In such an approach, each bin of a histogram represents a separate search channel, with its own signal and background acceptances and uncertainties. Therefore, the method for setting a limit described above is not directly applicable to our search, but it can be generalized for our case [96].

In the case of a binned distribution of the discriminant variable, with a total number of bins $N$, the $k^{t h}$ bin contains $n_{k}$ observed events and $s \epsilon_{k}+b_{k}$ expected events. Here, $s$ is the total number of expected signal events, $\epsilon_{k}$ and $b_{k}$ are the signal acceptance and the amount of background events in $k^{\text {th }}$ bin. All of the $\epsilon_{k}$ and $b_{k}$ have uncertainties and are considered "nuisance parameters". Similar to the procedure above, they are assigned priors, that may be correlated, and the joint prior is written as:

$$
\pi\left(\epsilon_{1}, b_{1}, \ldots, \epsilon_{N}, b_{N}\right)
$$


and the marginalized posterior for $s$ is proportional to:

$$
\pi(s) \int_{2 N} \ldots \int \pi\left(\epsilon_{1}, b_{1}, \ldots, \epsilon_{N}, b_{N}\right)\left[\prod_{k=1}^{N} \frac{e^{-\left(s \epsilon_{k}+b_{k}\right)}\left(s \epsilon_{k}+b_{k}\right)^{n_{k}}}{n_{k} !}\right] d \epsilon_{1} d b_{1} \ldots d \epsilon_{N} d b_{N}
$$

As mentioned above, we assume a flat prior $\pi(s)$ for the Higgs boson signal in this search. In order to obtain the limit on the signal, the posterior is integrated, as in Eq. 8.5. The marginalization of the posterior is done using Monte Carlo integration, by generating $M$ random $\left(\epsilon_{1}, b_{1}, \ldots, \epsilon_{N}, b_{N}\right)$ vectors ("ensemble"), according to their priors, and averaging over $M$. For results presented in this analysis, we used $M=$ 50000. The software we used takes into account the correlations between nuisance parameters when generating the random vectors. Since some of the uncertainties may result in shape variations of the $\mathrm{ANN}_{\mathrm{SIG}}$ distribution (such as the JES uncertainty), the histograms are interpolated within their shape uncertainties, when generating the random sets. Shape uncertainties are provided as inputs to the MCLIMIT package, in addition to the nominal histogram shapes. To obtain the upper limit $s_{u}$, the marginalized posterior is integrated over $s$ using numerical integration. The procedure is repeated $n$ times, generating a new ensemble for each repetition, and averaged over $n$ ( $n=10000$ for the results presented in this analysis). Since the value of the Higgs boson mass is unknown, we perform this procedure using different hypothesis for the mass, $m_{H}$. Each mass hypothesis is described with a Monte Carlo simulation of the Higgs boson production with a mass $m_{H}$.

The sensitivity of the search is quoted using the expected limit, where it is assumed that there is no signal of Higgs boson production. Therefore, the numbers of events in each bin $n_{k}$ are set equal to the number of predicted background events $b_{k}$. This step is done before opening the signal box in order to optimize the sensitivity of the search by optimizing the quantity $s_{u}^{e x p}$. 
In order to facilitate the comparisons of the results with the SM expectations, the upper limit $s_{u}$ on the number of Higgs boson events produced can be translated into a scaling factor $R$ :

$$
R=\frac{s_{u}}{\sum_{k=1}^{N} s \epsilon_{k}}=\frac{\sigma_{u}}{\sigma_{S M}(Z H / W H)}
$$

that relates the excluded cross-section with the cross-section expected from SM. The $R_{o b s}$ values are calculated using the number of events observed in CDF data, and the $R_{\exp }$ values correspond to the sensitivity of the search, obtained using the $s_{u}^{e x p}$. The values of $R_{o b s} \leq 1$ would indicate that the Higgs boson of a particular mass $m_{H}$ is excluded at $95 \%$ C.L.

\subsection{Results}

As shown in Section 8.3, we find good agreement between experimental data and the standard model predictions. Therefore we place 95\% confidence level upper limits on $\sigma_{V H} \cdot \mathcal{B} r(H \rightarrow b \bar{b})$. We use the Bayesian method described above, which takes into account the correlations between the systematic uncertainties. We test several Higgs boson mass hypotheses and place limits for masses ranging from $105 \mathrm{GeV} / c^{2}$ to $150 \mathrm{GeV} / c^{2}$, which are summarized in Table 8.7. The errors on $R_{\exp }$ represent the intervals that contain $68 \%$ of the $n$ trials, as described above. Figure 8.9 shows the expected and observed limits as a ratio to the SM cross section.

We obtain the observed (expected) limit of 6.9 (5.6) times the standard model prediction at $m_{H}=115 \mathrm{GeV} / c^{2}$. The effect of systematic uncertainties worsens the limits by $\sim 15-20 \%$. 
Table 8.7: The combined 95\% C.L. upper limits on cross section time $H \rightarrow b \bar{b}$ branching fraction when the Higgs boson is produced in association with weak boson. The last two columns give the ratio of the expected and observed limits with respect to the SM cross section.

\begin{tabular}{|c|c|c|c|c|}
\hline $\begin{array}{c}\text { Higgs boson mass } \\
\left(\mathrm{GeV} / c^{2}\right)\end{array}$ & $\begin{array}{c}\text { Expected } \\
(\mathrm{pb})\end{array}$ & $\begin{array}{c}\text { Observed } \\
(\mathrm{pb})\end{array}$ & $\begin{array}{c}\text { Ratio Expected } \\
R_{\text {exp }}\end{array}$ & $\begin{array}{c}\text { Ratio Observed } \\
R_{\text {obs }}\end{array}$ \\
\hline \hline 105 & 1.5 & 1.7 & $4.7_{-1.4}^{+2.0}$ & 5.5 \\
110 & 1.3 & 1.5 & $4.9_{-1.4}^{+2.1}$ & 5.8 \\
115 & 1.2 & 1.5 & $5.6_{-1.6}^{+2.4}$ & 6.9 \\
120 & 1.2 & 1.5 & $7.2_{-2.1}^{+2.9}$ & 8.9 \\
125 & 1.1 & 1.6 & $8.4_{-2.4}^{+3.6}$ & 11.9 \\
130 & 1.0 & 1.4 & $10.3_{-2.9}^{+4.3}$ & 14.4 \\
135 & 1.0 & 1.2 & $13.8_{-3.9}^{+5.8}$ & 16.2 \\
140 & 0.9 & 1.0 & $18.6_{-5.4}^{+7.8}$ & 21.0 \\
145 & 0.9 & 1.0 & $28.6_{-8.2}^{+11.8}$ & 33.4 \\
150 & 0.8 & 1.0 & $43.3_{-12.4}^{+19.0}$ & 49.8 \\
\hline
\end{tabular}




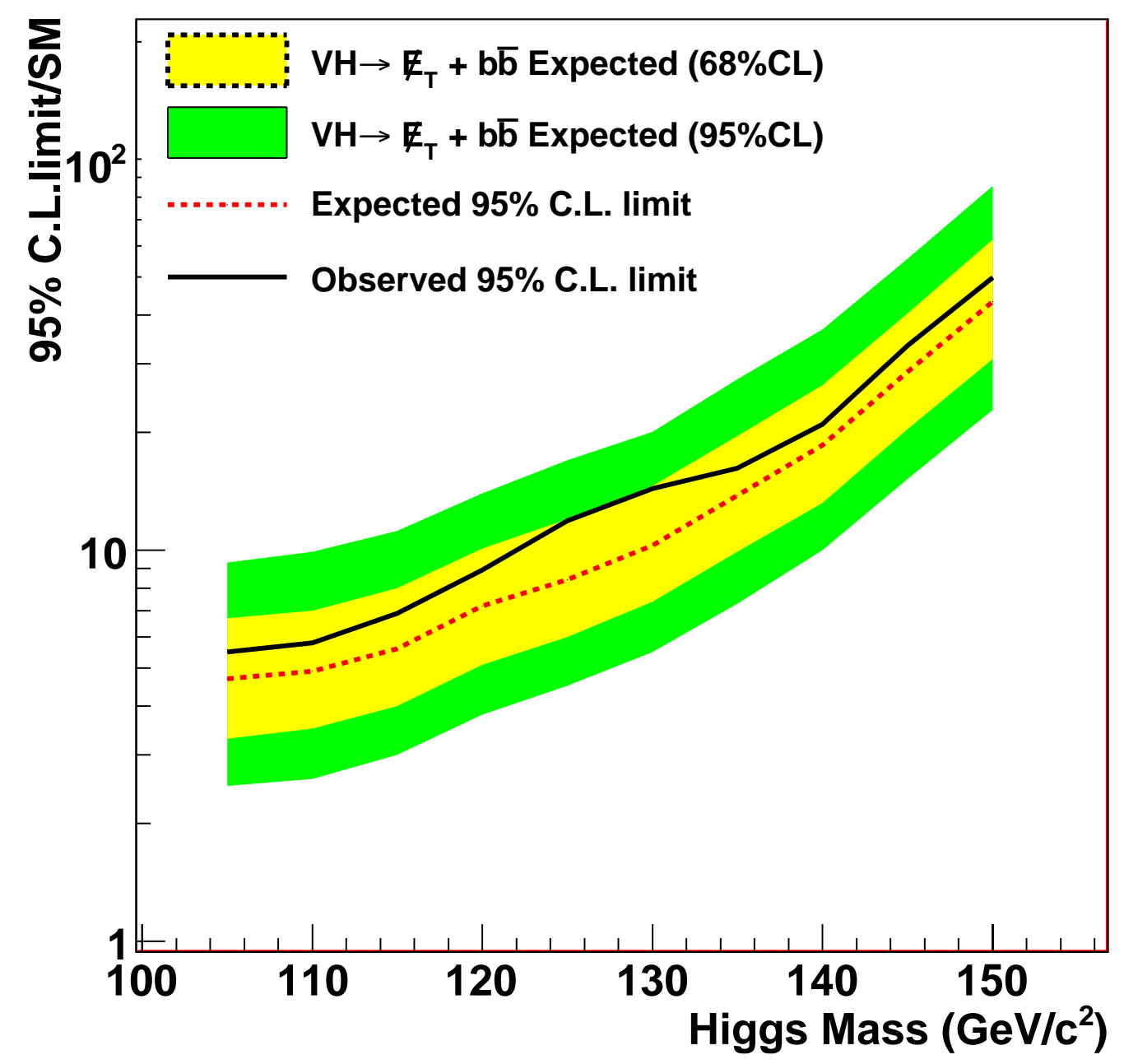

Figure 8.9.: Expected and observed limits as a ratio compared to Standard Model cross sections. Black line represents the $R_{o b s}$ values and the red dotted line shows the $R_{\text {exp }}$ values at different masses 


\subsection{Conclusion}

In summary, we have performed a direct search for the SM Higgs boson decaying into $b$-jet pairs using data with integrated luminosity of $2.1 \pm 0.13 \mathrm{fb}^{-1}$ accumulated in Run II of the Tevatron using the CDF II detector. A data-driven method to determine the multi-jet background and a novel event selection using $\mathrm{ANN}_{\mathrm{MJ}}$ have been developed. As a result of these improvements we have doubled the signal acceptance by relaxing many kinematic and topological cuts. We also use two $\mathrm{ANN}_{\mathrm{SIG}}$ to separate the signal from the backgrounds and achieve significant improvements in Higgs boson sensitivity over previous analyses in this decay mode [74]. The observed event kinematics and $\mathrm{ANN}_{\mathrm{SIG}}$ show no significant excess above SM predictions, and 95\% C.L. upper limits are placed on the Higgs boson production cross-section. The combination of all improvements described above increase the sensitivity of this search by a factor of 3 with respect to [74], which is about 2 times better than expected by the larger data set alone. The improvement of the limits using our approach of two ANNs is a factor of 1.3 compared to a fit of the dijet mass distribution alone.

The sensitivity of each individual search at the Tevatron is low, especially if the Higgs boson is light (below $\sim 135 \mathrm{GeV} / c^{2}$ ), which is the region favored by electroweak fits [32]. In order to maximize the experimental reach of the Tevatron, the results from various, statistically independent analyses, are combined and then the results from the CDF and D0 are also combined. This allows to increase the sensitivity of searches at the Tevatron by doubling the amount of analyzed data. The most recent result of the Tevatron combination [97] is presented in Fig. 8.10, which shows that the experiments at the Tevatron are getting close in sensitivity to exclude the Higgs boson if it does not exist or see the first evidence of its existence in the low mass. As a result of this combination, the Tevatron experiments have achieved a sensitivity of 2.4 times the SM prediction at $m_{H}=115 \mathrm{GeV} / c^{2}$. Furthermore, the Tevatron combination has excluded the Higgs boson in the mass range between $m_{H}=160 \mathrm{GeV} / c^{2}$ and $m_{H}=170 \mathrm{GeV} / c^{2}$ at $95 \%$ C.L. 


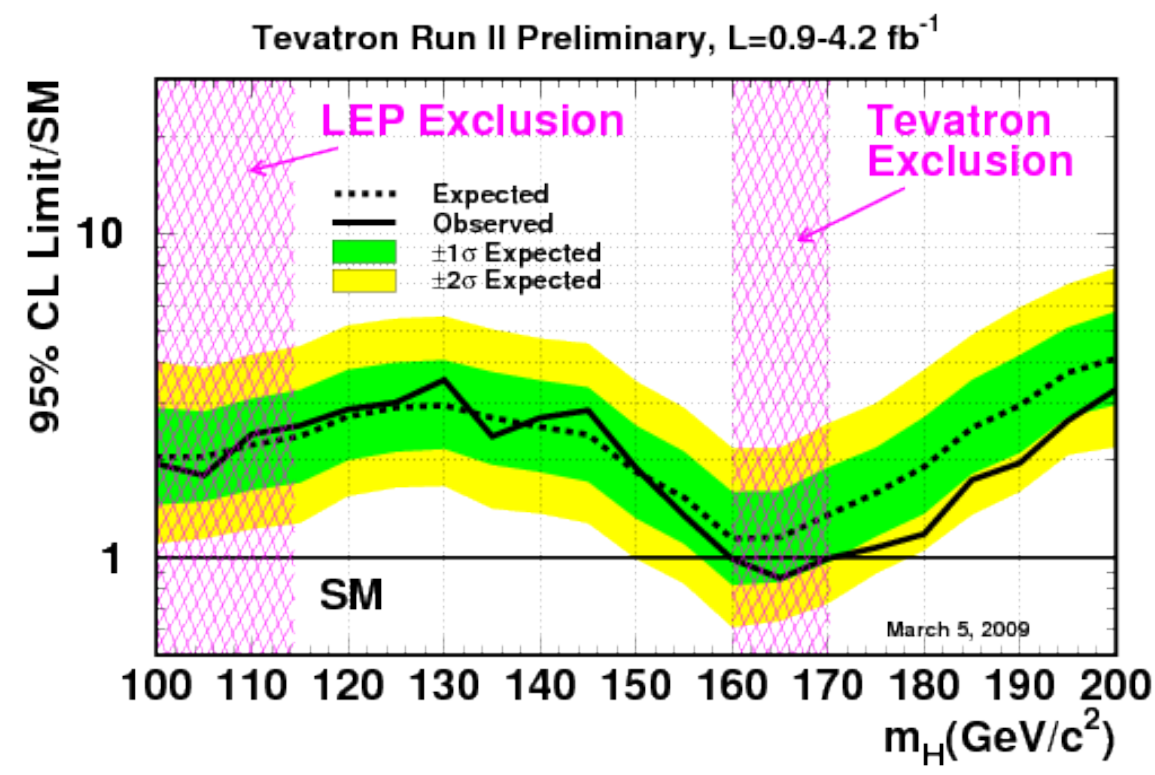

Figure 8.10.: The Tevatron combined 95\% C.L. upper limit as a function of the Higgs boson mass between 100 and $200 \mathrm{GeV} / c^{2}$ Solid black: observed limit/SM; Dashed black: median expected limit/SM. Colored bands: $\pm 1,2 \sigma$ distributions around median expected limit.

Figure 8.11 shows the CDF combined limit. The contribution of the results of the search in MET+Jets channel are labeled as "WH+ZH $\rightarrow$ bbMET". It can be seen that the results presented in this dissertation are among the most sensitive channels in the low mass region.

The experimental sensitivity for directly observing the Higgs boson at the Tevatron is steadily improving. Due to the novel analysis techniques, the sensitivity of the Tevatron Higgs boson searches increases faster than the expectation from larger accumulated dataset alone. Many improvements to the searches of low mass Higgs boson are currently under way. The improvements in the trigger system will allow a more efficient data acquisition with high signal acceptance rates. Improvements in the 


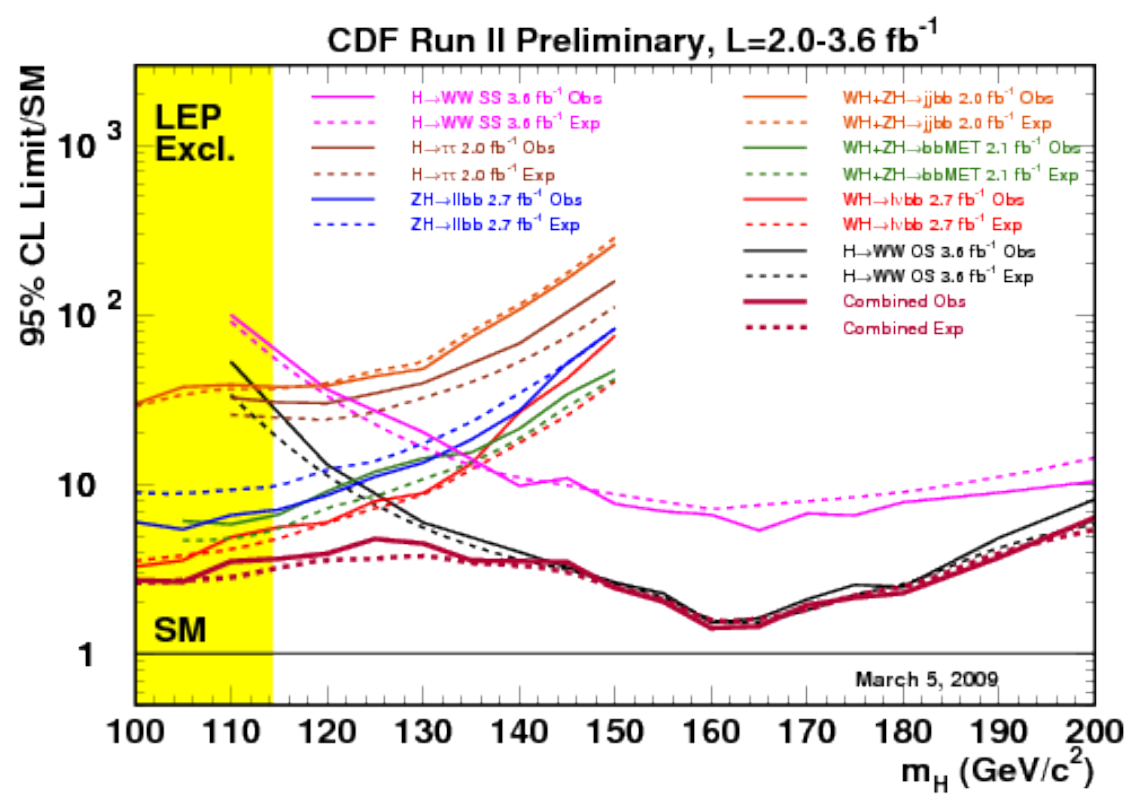

Figure 8.11.: The CDF combined 95\% C.L. upper limit as a function of the Higgs boson mass between 100 and $200 \mathrm{GeV} / c^{2}$. Solid lines indicate the observed upper limit for each channel and combined result (dark red). Dashed lines indicate the median expected upper limit for each channel and the combined result (dark red).

$b$-tagging efficiency, which is crucial for the Higgs boson searches, are another avenue for improvements. The CDF collaboration has recently developed a new $b$-tagging algorithms based on neural network techniques, that allows to considerably improve the $b$-tagging efficiency. Current sensitivity in the low mass region is mostly limited by the statistical uncertainty of the accumulated data. However, the systematic uncertainties will have a larger impact once a larger dataset is collected. Tevatron is expected to deliver $\sim 8 \mathrm{fb}^{-1}$ per experiment by the end of 2010 . Therefore, efforts are ongoing to reduce the uncertainties on the background sources. Improvements in the analysis technique, such as more sophisticated event selection, will further increase the reach of the Higgs boson searches. 
Many analysis techniques that were developed and successfully used in other analyses at CDF are now being implemented also in the Higgs searches. An example is the use of matrix element calculations to predict the probability of an event being signal- or background-like, which was previously used in the measurements involving top quarks [98]. Similarly, methods developed for the Higgs searches are being tested in other analysis, such as the measurement of the cross section of the electroweak production of top quarks. Using the techniques developed for the Higgs boson search, we performed the first measurement of this process in events with $\mathbb{E}_{T}$ and jets [99] at $\mathrm{CDF}$, achieving observed signal significance of $2.1 \sigma$. Our analysis provided an important contribution to the combined sensitivity of the single top measurement at CDF. The most recent combination of CDF results, which included the MET+Jets analysis, resulted in the first observation of this rare process [100] at the significance of $5.0 \sigma$.

The Fig. 8.12 shows the progress in improvements of the combined CDF sensitivity and the projections of expected improvements. It can be readily seen that the CDF sensitivity for the Higgs boson has been increasing at a much faster rate than from a larger accumulated dataset alone. The band represents the projected sensitivity, assuming further improvements in the techniques used in the Higgs boson searches. The lower border of the band assumes an improvement of a factor of 1.5 with respect to the current analysis performed at CDF. The combination of the CDF and D0 results will allow to further increase the sensitivity of Higgs searches, by doubling the available luminosity. Achieving these improvements will mean that with the full luminosity available at the end of running in 2010, the Higgs boson searches at the Tevatron will be sensitive to the SM production rates of the Higgs boson over a wide mass range. 


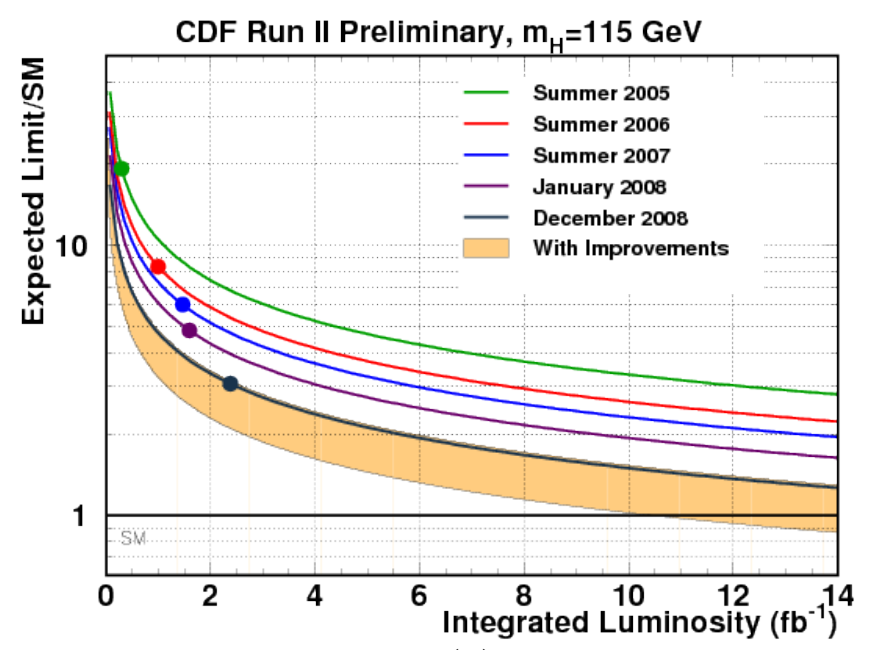

(a)

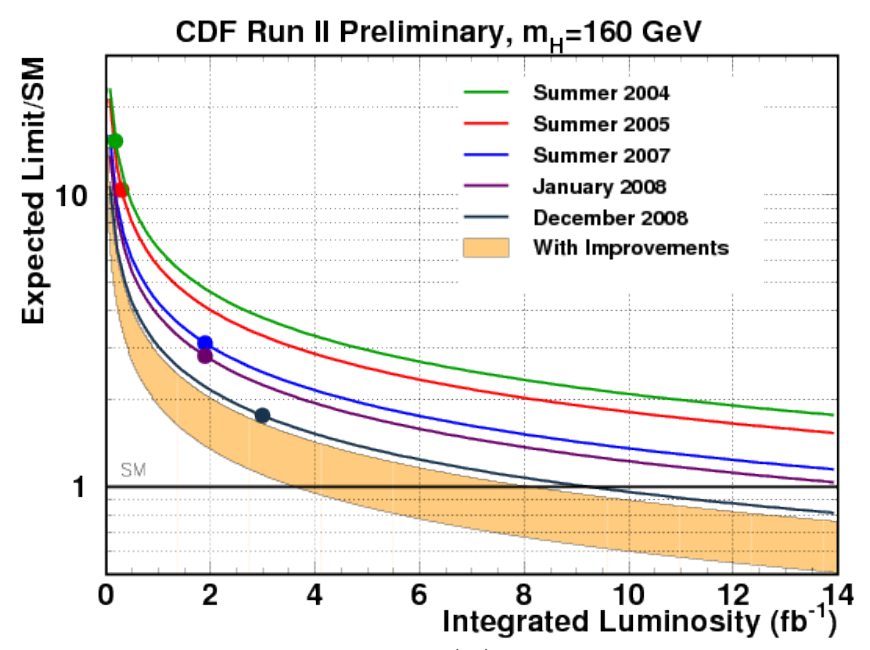

(b)

Figure 8.12.: The projected sensitivity of CDF searches for the Higgs boson (a) for $m_{H}=115 \mathrm{GeV} / c^{2}$ and (b) for $m_{H}=160 \mathrm{GeV} / c^{2}$. The combination of CDF and D0 searches will allow to further increase the sensitivity of the Higgs searches, by doubling the analyzed data. 
LIST OF REFERENCES 


\section{LIST OF REFERENCES}

[1] J.J. Thomson. Cathode rays. Philos. Mag., 44:293, 1897.

[2] E. Rutherford. The Scattering of $\alpha$ and $\beta$ Particles by Matter and the Structure of the Atom. Philos. Mag., 21:669-688, 1911.

[3] A.I.Veinstein,. Sov. Phys. Usp., 23:429, 1980.

[4] F.Halzen, A.Martin. Quarks and Leptons: An Introductory Course in Modern Particle Physics. John Wiley \& Sons, 1984.

[5] D.H.Perkins. Introduction to High Energy Physics. Cambridge University Press, 2000.

[6] L.H.Ryder. Quantum Field Theory. Cambridge University Press, 1996.

[7] M.E.Peskin, D.V.Schroeder. An Introduction To Quantum Field Theory. Westview Press, 1995.

[8] G. 't Hooft. Renormalizable lagrangians for massive Yang-Mills fields. Nucl. Phys., B35:167, 1971.

[9] G. 't Hooft, M.Veltman. Regularization and renormalization of gauge fields. Nucl. Phys., B44:189, 1972.

[10] C. Amsler et al. Particle data group. Phys. Lett., B667:1, 2008.

[11] E.Fermi,. Nuovo Cim., 11:1, 1934.

[12] R. P. Feynman and M. Gell-Mann,. Phys. Rev., 109:193, 1958.

[13] R. E. Marshak and E. C. G. Sudarshan,. Phys. Rev., 109:1860, 1958.

[14] S. A. Bludman,. Nuovo Cim., 9:433, 1958.

[15] F. J. Hasert et al.,. Phys. Lett. B., 46:121, 1973.

[16] S. L. Glashow,. Nucl. Phys., 22:579, 1961.

[17] P.W.Higgs,. Phys. Lett., 12:132, 1964.

[18] S.Weinberg,. Phys.Rev.Lett., 19:1264, 1967.

[19] A. Salam. Elementary particle theory. In N. Svartholm, editor, Proc. 8th Nobel Symp. Almquist and Forlag A. B., Stockholm, 1968.

[20] UA1 Collaboration, G.Arnison et al.,. Phys. Lett., 126B:398, 1983. 
[21] UA2 Collaboration, P.Bagnaia et al.,. Phys. Lett., 129B:130, 1983.

[22] UA1 Collaboration, G.Arnison et al.,. Phys. Lett., 122B:103, 1983.

[23] UA2 Collaboration, P.Banner et al.,. Phys. Lett., 129B:476, 1983.

[24] Hideki Yukawa. On the interaction of elementary particles. Proc. Phys. Math. Soc. Jap., 17:48-57, 1935.

[25] D. J. Gross and Frank Wilczek. Ultraviolet behaviour of non-abelian gauge theories. Phys. Rev. Lett., 30:1343-1346, 1973.

[26] H. David Politzer. Setting the scale for predictions of asymptotic freedom. Phys. Rev., D9:2174-2175, 1974.

[27] M. Quiros, J. A. Casas, J. R. Espinosa,. Phys. Lett. B, 342:171, 1995.

[28] Hambye, Thomas and Riesselmann, Kurt . Matching conditions and Higgs boson mass upper bounds reexamined. Phys. Rev. D, 55(11):7255-7262, Jun 1997.

[29] M. Sher,. Phys. Rep., 179:273, 1989.

[30] G. Altarelli and G. Isidori,. Phys. Lett. B, 337:141, 1994.

[31] M. Veltman,. Acta. Phys. Pol., B8:475, 1977.

[32] LEP Electroweak Working Group:. http://lepewwg. web.cern.ch/LEPEWWG/.

[33] The LEP Higgs Working Group. Search for the standard model Higgs boson at LEP. Phys. Lett B, 565:61-75, 2003.

[34] T. Hahn and S. Heinemeyer and F. Maltoni and G. Weiglein and S. Willenbrock. SM and MSSM Higgs Boson Production Cross Sections at the Tevatron and the LHC, 2006.

[35] A. Djouadi and J. Kalinowski and M. Spira. HDECAY: a Program for Higgs Boson Decays in the Standard Model and its Supersymmetric Extension. Comput. Phys. Commun., 108:56-74, 1998.

[36] Tevatron Run II Handbook. http://www-bd.fnal.gov/lug/runII_handbook/ RunII_index.html.

[37] TeVI Group. Design Report Tevatron 1 project. FERMILAB-DESIGN-1984-01.

[38] R. Blair et al., (CDF Collaboration), The CDF-II Detector Technical Design Report, Fermilab- Pub-96/390-E (1996).

[39] Christopher S. Hill. Operational experience and performance of the CDFII silicon detector. Nucl. Instrum. Meth. A, 530(1-2):1-6, 2004.

[40] P. Azzi. The CDF silicon detector upgrade. Nucl. Instrum. Meth. A, 419(23):532-537, 1998.

[41] Alan Sill. CDF Run II silicon tracking projects. Nucl. Instrum. Meth. A, $447(1-2): 1-8,2000$. 
[42] A. Affolder et al. Intermediate silicon layers detector for the CDF experiment. Nucl. Instrum. Meth. A, 453(1-2):84-88, 2000.

[43] A. Affolder et al. CDF Central Outer Tracker. Nucl. Instrum. Meth. A, 526(3):249 - 299, 2004.

[44] D. Acosta et al. A Time-of-Flight detector in CDF-II. Nucl. Instrum. Meth. A, 518(1-2):605 - 608, 2004.

[45] H. Minemura et al. Construction and testing of a $3 \mathrm{~m}$ diameter $\times 5 \mathrm{~m}$ superconducting solenoid for the fermilab collider detector facility (CDF). Nucl. Instrum. Meth. A, 238(1):18 - 34, 1985.

[46] L. Balka et al. The CDF central electromagnetic calorimeter. Nucl. Instrum. Meth. A, 267(2-3):272 - 279, 1988.

[47] S. Kuhlmann et al. The CDF calorimeter upgrade for Run IIb. Nucl. Instrum. Meth. A, 518(1-2):39-41, 2004.

[48] G. Apollinari et al. Shower maximum detector for the CDF plug upgrade calorimeter. Nucl. Instrum. Meth. A, 412(2-3):515 - 526, 1998.

[49] S. Bertolucci et al. The CDF central and endwall hadron calorimeter. Nucl. Instrum. Meth. A, 267(2-3):301-314, 1988.

[50] G. Ascoli et al. CDF central muon detector. Nucl. Instrum. Meth. A, 268(1):33 $-40,1988$.

[51] C.M. Ginsburg. Eur. Phys. J, 33, Supplement 1:s1002-s1004, 2004.

[52] D. Acosta et al. The performance of the CDF luminosity monitor. Nucl. Instrum. Meth. A, 494(1-3):57-62, 2002.

[53] Abe, F. et al. Measurement of the antiproton-proton total cross section at $\sqrt{s}=546$ and $1800 \mathrm{GeV}$. Phys. Rev. D, 50(9):5550-5561, 1994.

[54] R. Downing et al. Track extrapolation and distribution for the CDF-II trigger system. Nucl. Instrum. Meth. A, 570(1):36 - 50, 2007.

[55] B. Ashmanskas et al. The CDF Silicon Vertex Trigger. Nucl. Instrum. Meth. A, 518(1-2):532 - 536, 2004.

[56] R. Brun and F. Rademakers. ROOT - An object oriented data analysis framework. Nucl. Instrum. Meth. A, 389(1-2):81 - 86, 1997.

[57] R. Colombo et al. The CDF Computing and Analysis System: First Experience. In Proceedings CHEP2001.

[58] P. Murat. STNTUPLE: ROOT-based microDST analysis system for CDF. In Proceedings CHEP2001.

[59] F.D. Snider. Tracking at CDF: Algorithms and experience from Run I and Run II. Nucl. Instrum. Meth. A, 566(1):133 - 141, 2006.

[60] C. Hays et al. Inside-out tracking at CDF. Nucl. Instrum. Meth. A, 538(1-3):249 $-254,2005$. 
[61] F. Abe et al. Topology of three-jet events in $p \bar{p}$ collisions at $\sqrt{s}=1.8 \mathrm{TeV}$. Phys. Rev. D, 45(5):1448-1458, 1992.

[62] A. Bhatti et al. Determination of the jet energy scale at the Collider Detector at Fermilab. Nucl. Instrum. Meth. A, 566(2):375 - 412, 2006. and referecnes therein.

[63] H1 Collaboration, C. Adloff et al.,. Z. Phys. C, 74:221-235, 1997.

[64] OPAL Collaboration, G. Abbiendi et al.,. Eur. Phys. J. C, 12:567-586, 2000.

[65] A. Mehta. Jet Resolution Studies of the H1 Method to Combine Tracks and Calorimeter Towers. CDF internal note 7468 (unpublished).

[66] CDF and D0 Collaborations. Results of the Tevatron Higgs Sensitivity Study. http://tevewwg.fnal.gov/higgs/hss03d0cdf.pdf.

[67] A. Apresyan, D. Bortoletto, F. Margaroli, O. Gonzalez, S. M. Wang. Tag Rate Matrix method to estimate QCD background from data in VH search in $\mathrm{MET}+b$ - jets signature. CDF internal note 9275 (unpublished).

[68] B. Kilminster, B. Parks, R. Hughes, B. Winer. Using track-based discriminants for distinguishing Higgs from QCD backgrounds. CDF internal note 8978 (unpublished).

[69] D. Acosta, et al. Measurement of the $t \bar{t}$ production cross section in $p \bar{p}$ collisions at $\sqrt{s}=1.96 \mathrm{TeV}$ using lepton + jets events with secondary vertex $b$-tagging. Phys. Rev. D, 71(5):052003, Mar 2005.

[70] A. Abulencia, et al. Measurement of the $t \bar{t}$ production cross section in $p \bar{p}$ collisions at $\sqrt{s}=1.96 \mathrm{TeV}$ using lepton + jets events with jet probability b-tagging. Phys. Rev. D, 74:072006, October 2006.

[71] A. Bhatti et al. The CDF level 2 calorimetric trigger upgrade. Nucl. Instrum. Meth. A, 598(1):331-333, 2009.

[72] D. Acosta, et al.,. CDF internal note 7410 (unpublished).

[73] J. Antos et al. Data processing model for the CDF experiment. IEEE Trans. Nucl. Sci., 53:2897-2906, 2006.

[74] Viktor Veszpremi. Search for the standard model Higgs boson in protonantiproton collisions at a center-of-mass energy of $1.96 \mathrm{TeV}$. FERMILABTHESIS-2007-30.

[75] O. Gonzalez, M. Vidal. Trigger efficiency of the MET+JETS sample for the $2 \mathrm{fb}^{-1}$ dataset. CDF internal note 9355 (unpublished).

[76] O. Gonzalez, M. Vidal. Official Recovery of Events with Saturated Towers using the JET100 sample (for the MET dataset). CDF internal note 9231 (unpublished).

[77] Torbjorn Sjostrand et al. High-energy-physics event generation with PYTHIA 6.1. Comput. Phys. Commun., 135:238-259, 2001. 
[78] K. A. Assamagan et al. The Higgs working group: Summary report 2003. 2004.

[79] D. Acosta et al. (CDF Collaboration). First Measurements of Inclusive $W$ and $Z$ Cross Sections from Run II of the Fermilab Tevatron Collider. Phys. Rev. Lett., 94:091803, 2005.

[80] A. Abulencia et al. (CDF Collaboration). Measurement of the $b$ jet cross section in events with a $Z$ boson in $p \bar{p}$ collisions at $\sqrt{s}=1.96 \mathrm{TeV}$. Phys. Rev. D, 74:032008, 2006.

[81] J. M. Campbell and R. K. Ellis,. Phys. Rev. D, 62:114012, 2000.

[82] J. Alwall et al. MadGraph/MadEvent v4: the new web generation. J. High Energy Phys., 2007(09):028, 2007.

[83] CDF collaboration. Combination of CDF top quark pair production cross section measurements with $2.8 \mathrm{fb}^{-1}$. CDF public note 9448,2008.

[84] Z. Sullivan. Understanding single-top-quark production and jets at hadron colliders. Phys. Rev. D, 70(11):114012, Dec 2004.

[85] B. W. Harris et al. Fully differential single-top-quark cross section in next-toleading order QCD. Phys. Rev. D, 66(5):054024, Sep 2002.

[86] W. McCulloch and W. Pitts. A logical calculus of the ideas immanent in nervous activity forms. Bulletin of Methematical Biophysics, 5:115-133, 1943.

[87] S. Haykin. Neural Networks: A Comprehensive Foundation (2nd Edition). Prentice Hall, July 1998.

[88] A. Hocker et al. TMVA - Toolkit for Multivariate Data Analysis. arXiv:physics/0703039v4.

[89] Chakravarti, Laha and Roy. Handbook of Methods of Applied Statistics. I:392394, 1967.

[90] Brandon Scott Parks. Search for the Higgs Boson in the $Z H \rightarrow v v b b$ Channel at CDF Run II. FERMILAB-THESIS-2008-18.

[91] H. L. Lai et al. Global QCD analysis of parton structure of the nucleon: CTEQ5 parton distributions. Eur. Phys. J., C12:375-392, 2000.

[92] Alan D. Martin, R. G. Roberts, W. James Stirling, and R. S. Thorne. Parton distributions: A New global analysis. Eur. Phys. J., C4:463-496, 1998.

[93] A. L. Read. Presentation of search results: the CLs technique. Journal of Physics G, 28(10):2693-2704, 2002.

[94] J. Heinrich et al. Interval estimation in the presence of nuisance parameters. 1. Bayesian approach. arXiv:physics/0409129, 2004.

[95] Thomas Junk. Confidence level computation for combining searches with small statistics. Nucl. Instrum. Meth. A, 434(2-3):435 - 443, 1999.

[96] J. Heinrich. Bayesian limit software: multi-channel with correlated backgrounds and efficiencies. CDF public note $758 \%$. 
[97] The TEVNPH Working Group. Combined CDF and DØ Upper Limits on Standard-Model Higgs-Boson Production with $4.2 \mathrm{fb}^{1}$ of data.

[98] A precision measurement of the mass of the top quark. Nature, 429(6229):638642, 062004.

[99] A. Apresyan, D. Bortoletto, F. Margaroli, K. Potamianos. First measurement of the single top production cross section in the MET plus jets sample. CDF public note 9650.

[100] T. Aaltonen (The CDF Collaboration). First Observation of Electroweak Single Top Quark Production. arXiv.org:0903.0885, 2009.

[101] A.Apresyan, D.Bortoletto, F.Margaroli, K.Potamianos, O. Gonzalez, M.Vidal, S. M. Wang. Event selection for Higgs search in the met plus jets sample. CDF internal note 9358 (unpublished). 
APPENDICES 


\section{Appendix A: Missing $E_{T}$ and Additional Triggers}

The MET35_\&_TWO_JETS that was used to collect the data in this analysis was first implemented on $22^{\text {nd }}$ of July, 2002. The requirements of this trigger were:

- MET35_\&_TWO_JETS:

- L1 : Require $\not_{T}>25 \mathrm{GeV}$.

- L2 : Two clusters with $E_{T}>10 \mathrm{GeV}$

- L3 : Require $\not_{T}>35 \mathrm{GeV}$.

Due to the improvements achieved by the Tevatron, the initial instantaneous luminosities delivered to CDF experiment were continuously increasing. As a result, the trigger rates at L2 were also increasing, due to large growth terms in jet based triggers, as was shown in Eq. 4.1. In order to maintain acceptable trigger rates, it was required to modify the trigger selections in 2005, when initial instantaneous luminosities above $10^{32} \mathrm{~cm}^{-2} \mathrm{~s}^{-1}$ became regular. The requirements for MET35_\&_TWO_JETS trigger changed on March 27, 2005, with the revised requirements:

- MET35_\&_CJET_\&_JET:

- L1 : Require $\notin_{T}>25 \mathrm{GeV}$.

- L2 : Two clusters with $E_{T}>10 \mathrm{GeV}$, with one cluster in $\eta<1.1$, i.e. central

- L3 : Require $\notin_{T}>35 \mathrm{GeV}$.

To preserve bandwidth, starting from September 2006 the MET35_\&_CJET_\&_JET trigger was collecting data only when instantaneous luminosity was below $1.9 \times 10^{32}$ $\mathrm{cm}^{-2} \mathrm{sec}^{-1}$, and from April 2007 this trigger was operating with a dynamic prescale ${ }^{1}$. Additional $\not_{T}$-based triggers used at CDF are the following:

- MET35_BJET (turned off above $1.5 \times 10^{32} \mathrm{~cm}^{-2} \mathrm{sec}^{-1}$ ):

\footnotetext{
${ }^{1} \mathrm{~A}$ trigger is referred to as operating at a prescale with a rate $\mathrm{P}$, if only $1 / \mathrm{P}$ fraction of events are recorded. Dynamic prescale: a feedback system that adjusts the prescale of triggers throughout a store according to the total trigger rate.
} 
- L1 : One cluster with $E_{T}>5 \mathrm{GeV}, \not_{T}>15 \mathrm{GeV}$ and two tracks with $p_{T}>2 \mathrm{GeV} / \mathrm{c}$.

- L2 : Two displaced tracks with $p_{T}>2 \mathrm{GeV}$ and minimum $\Delta \phi$ between tracks of $2^{\circ}$. One cluster with $E_{T}>15 \mathrm{GeV}$ that matches to a displaced track.

- L3: $\not_{T}>20 \mathrm{GeV}$ and two displaced tracks with $p_{T}>2 \mathrm{GeV}$

- MET45:

$-\mathrm{L} 1: \not \oplus_{T}>25 \mathrm{GeV}$

- L2: $\notin_{T}>35 \mathrm{GeV}$

- L3: $\notin_{T}>45 \mathrm{GeV}$

- MET25:

$-\mathrm{L} 1: \mathbb{E}_{T}>25 \mathrm{GeV}$

- L2 : prescale factor, which has increased throughout CDF II running from $P=100$ to $P=400$

- L3 : no requirements

Triggers used for the MET+Jets trigger efficiency studies:

- MUON_CMPU18:

- L1 : one muon candidate with $p_{T}>6 \mathrm{GeV} / \mathrm{c}$ and a matched track with $p_{T}>4 \mathrm{GeV} / \mathrm{c}$

- L2 : one muon candidate track with $p_{T}>15 \mathrm{GeV} / \mathrm{c}$

- L3 : one muon candidate with CMU and CMP hits and $p_{T}>18 \mathrm{GeV} / \mathrm{c}$

- JET20:

- L1 : one cluster with $E_{T}>5 \mathrm{GeV}$, prescale $P=50$

- L2 : one cluster with $E_{T}>15 \mathrm{GeV}$, prescale $P=25$ 
- L3 : one jet with $E_{T}>20 \mathrm{GeV}$

- JET50:

- L1 : one cluster with $E_{T}>5 \mathrm{GeV}$, prescale $P=50$

- L2 : one cluster with $E_{T}>40 \mathrm{GeV}$, prescale $P=2$

- L3 : one jet with $E_{T}>50 \mathrm{GeV}$

As it was described in Section 4.2.4, due to a hardware problem at Level 1 of the MET+Jets trigger, certain events with a single tower above $127 \mathrm{GeV}$, that should have been recorded by the trigger, were in fact discarded. We recover these events using the JET100 trigger:

- JET100:

- L1 : one cluster with $E_{T}>20 \mathrm{GeV}$

- L2 : one cluster with $E_{T}>90 \mathrm{GeV}$

- L3 : one jet with $E_{T}>100 \mathrm{GeV}$

The efficiency of the JET100 triggered sample is $100 \%$ for events which contain a tower with $E_{T}>127 \mathrm{GeV}$ at Level 1 . 


\section{Appendix B: Additional Distributions in Control Regions}

We utilize control regions to test our ability to model the background composition in the MET+Jets sample. Several control regions were defined in Sections 6.1 and 7.3. The modelling of different types of physics processes is tested in these control regions, allowing us to validate our model of the backgrounds that are expected to contribute to the signal region. Comparisons of the background model with the observed data in control regions were shown in Figures 6.1-6.6. In this chapter we show additional distributions of kinematic variables in the control regions. 

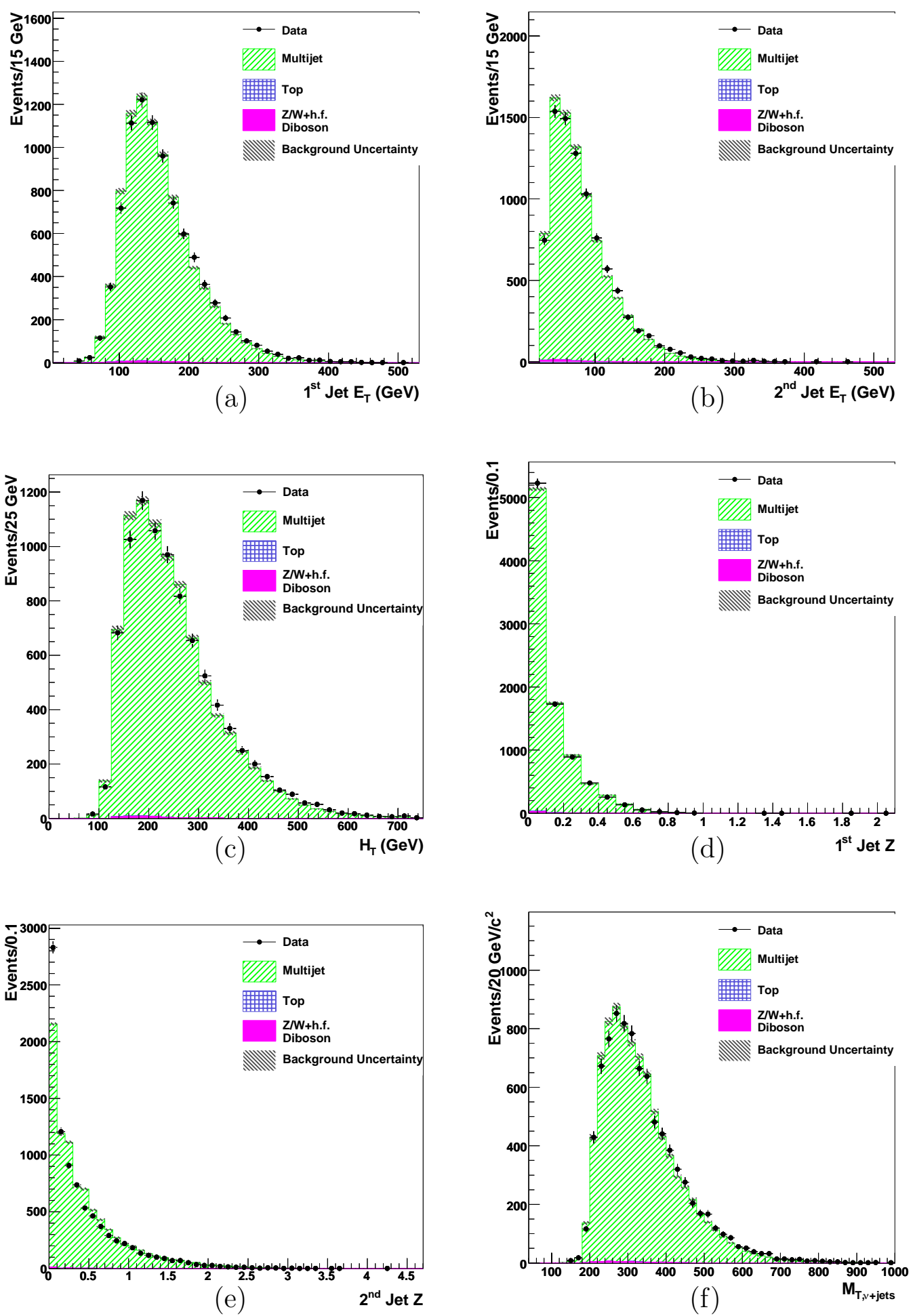

Figure B.1.: Kinematic distributions in CR1 for events with single $b$-tag: (a) $1^{\text {st }}$ jet $E_{T}$, (b) $2^{\text {nd }}$ jet $E_{T}$, (c) event $H_{T}$, (d) $1^{\text {st }}$ jet fragmentation function $Z$, (e) $2^{\text {nd }}$ jet fragmentation function $Z$, (f) invariant transverse mass of all jets and $\mathbb{E}_{T}$ 



(d)
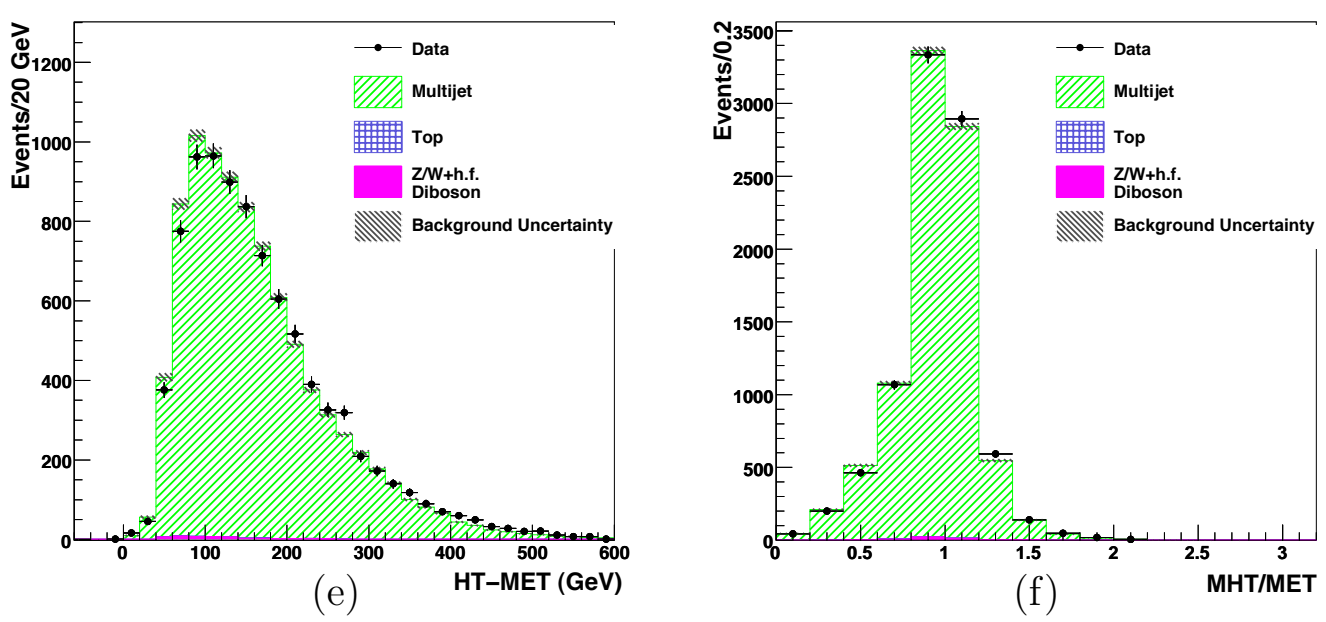

Figure B.2.: Kinematic distributions in CR1 for events with single b-tag: (a) minimum of the difference in $\phi$ between $\overrightarrow{\not p_{T}^{t r}}$ and each jet, (b) minimum of the difference in $\phi$ between $\vec{\epsilon}_{T}$ and each jet, (c) maximum of the difference in $R$ space between any two jets, (d) $H_{T}-\#_{T}$, (e) $H_{T}-\notin_{T}$, (f) $\not H_{T} / \notin_{T}$ 

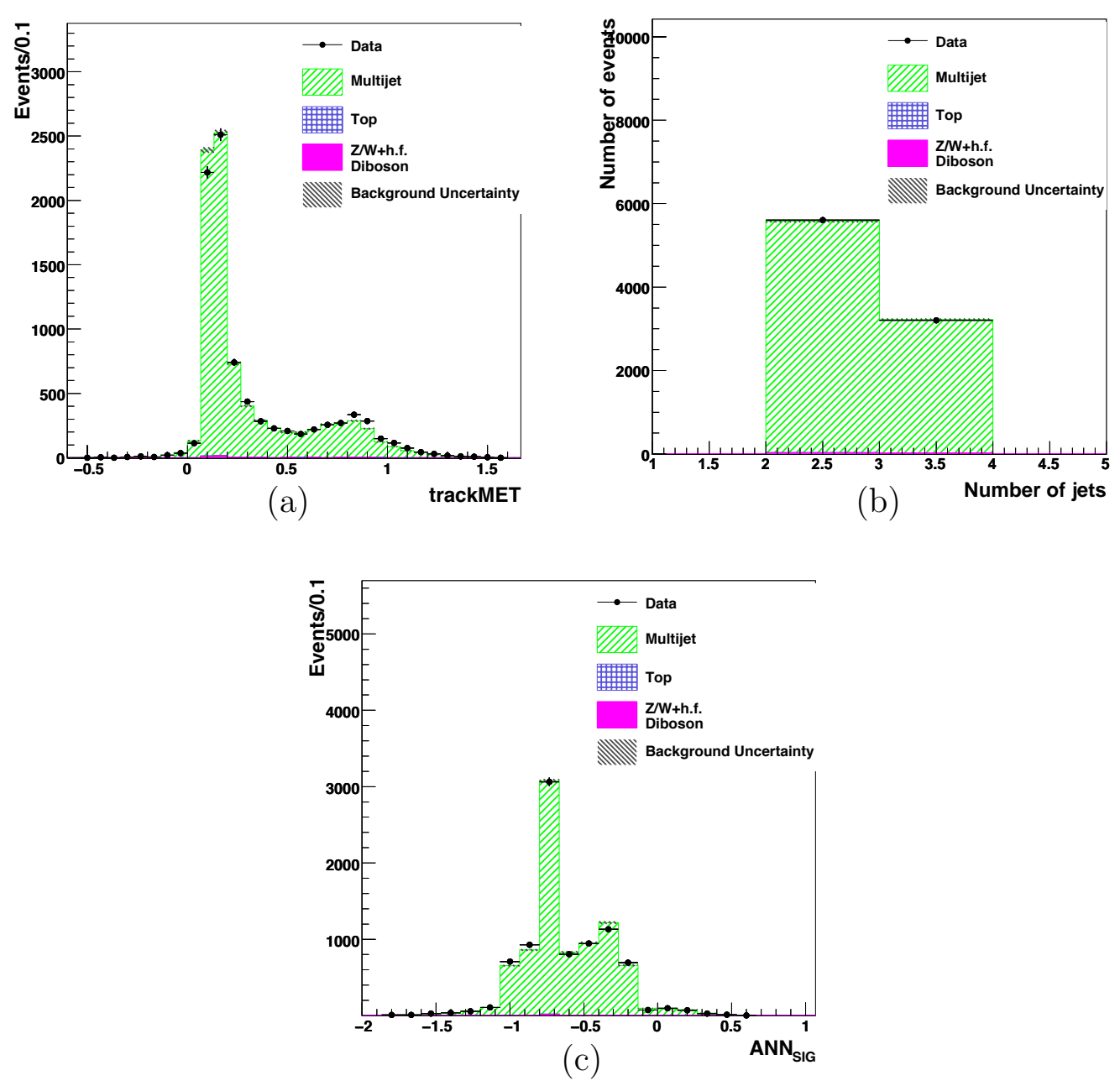

Figure B.3.: Kinematic distributions in CR1 for events with single $b$-tag: (a) output distribution of $\mathrm{ANN}_{\text {trackMET }}$, (b) number of jets, (d) output distribution of $\mathrm{ANN}_{\mathrm{SIG}}$ 



Figure B.4.: Kinematic distributions in CR2 for events with single b-tag: (a) $1^{\text {st }}$ jet $E_{T}$, (b) $2^{\text {nd }}$ jet $E_{T}$, (c) event $H_{T}$, (d) $1^{\text {st }}$ jet fragmentation function $Z$, (e) $2^{\text {nd }}$ jet fragmentation function $Z$, (f) event $\notin_{T}$ 


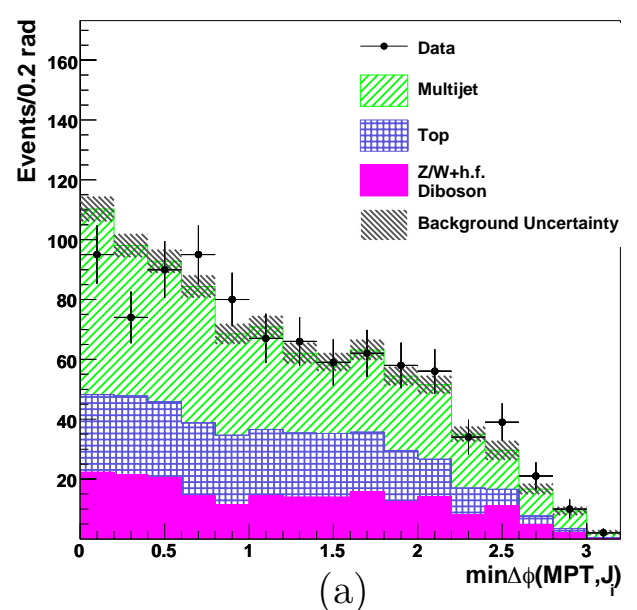

(a)
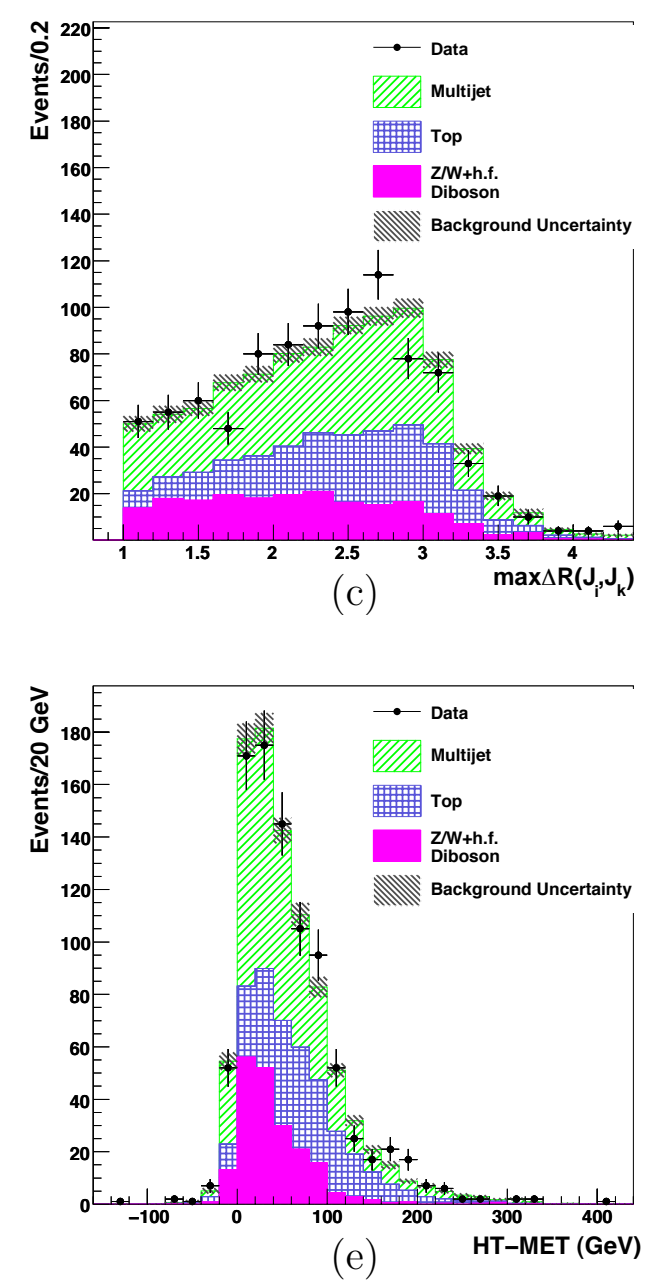
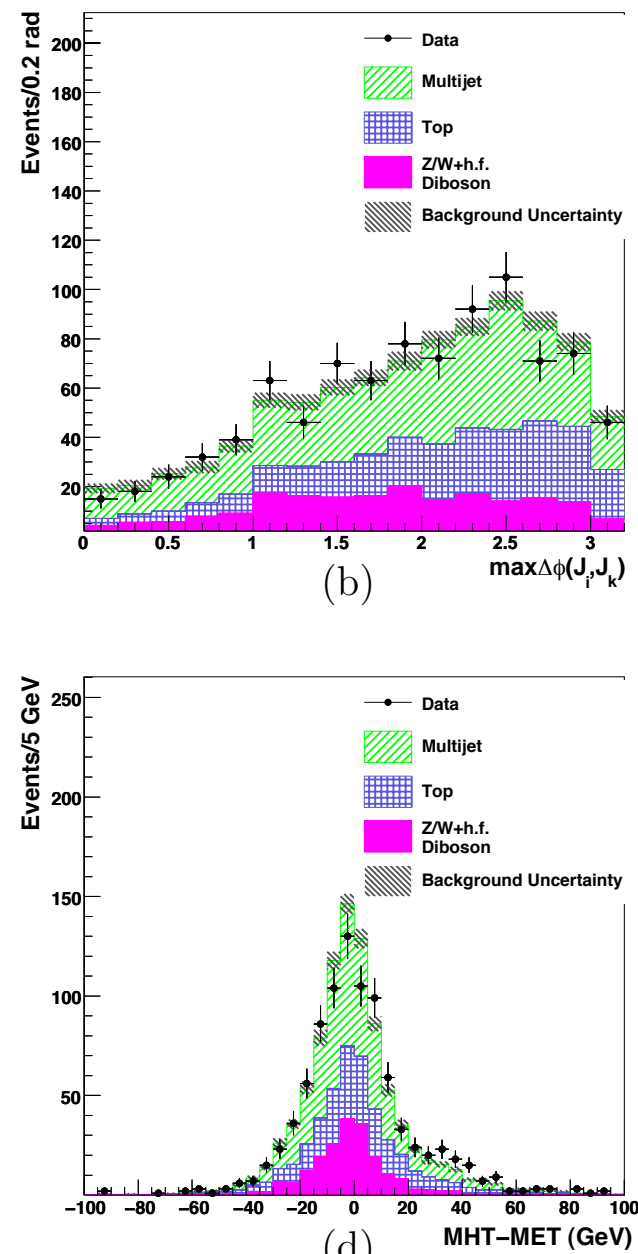

(d)

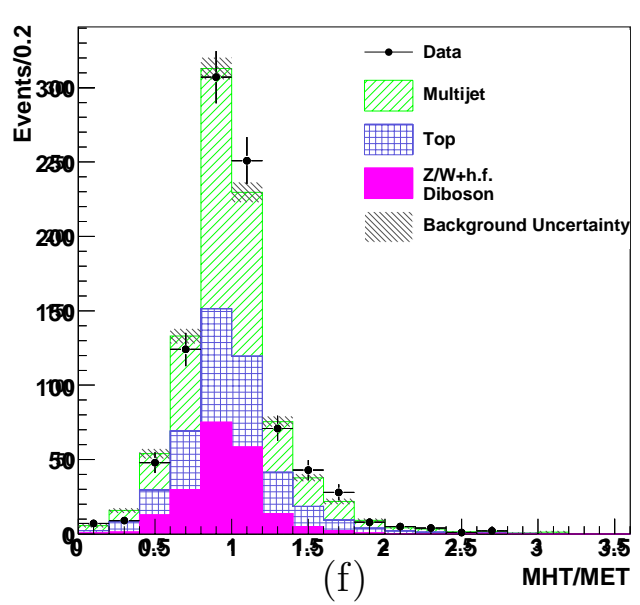

Figure B.5.: Kinematic distributions in CR2 for events with single b-tag: (a) minimum of the difference in $\phi$ between $\overrightarrow{\not P_{T}^{t r}}$ and each jet, (b) maximum of the difference in phi between two jets directions, (c) maximum of the difference in $R$ space between any two jets, (d) $\not_{T}-\not_{T}$, (e) $H_{T}-\mathscr{E}_{T}$, (f) $\not_{T} / \mathscr{E}_{T}$ 


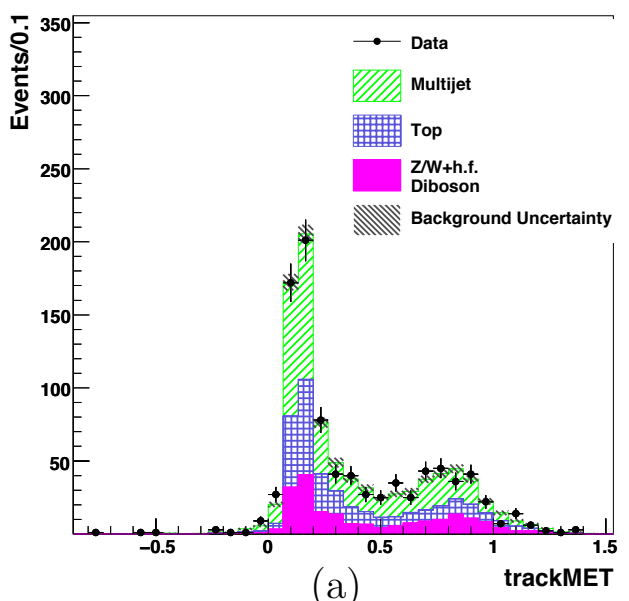

(a)

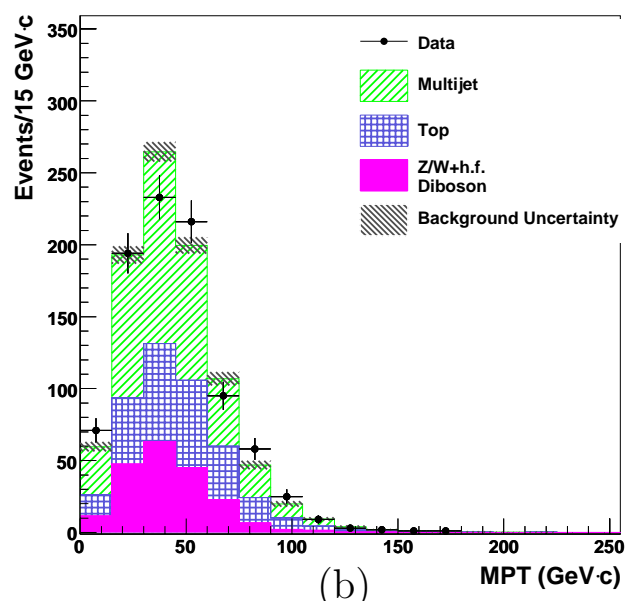

(b)

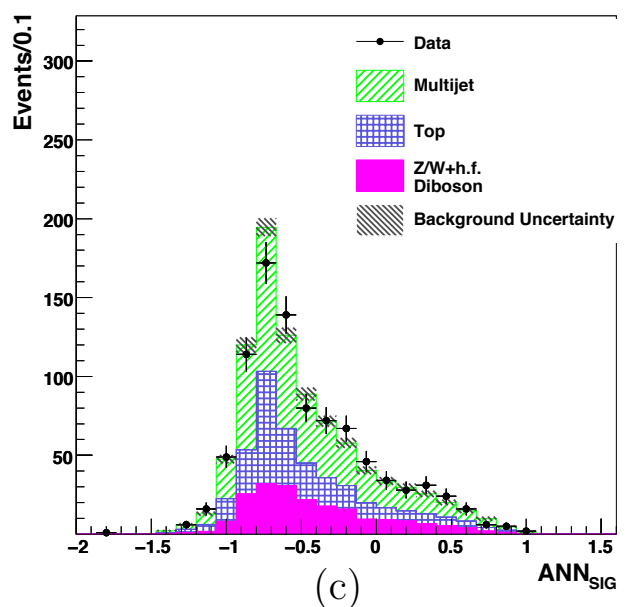

Figure B.6.: Kinematic distributions in CR2 for events with single b-tag: (a) output distribution of $\mathrm{ANN}_{\text {trackMET, }}$ (b) event $\not P_{T}^{t r}$, (d) output distribution of $\mathrm{ANN}_{\mathrm{SIG}}$ 

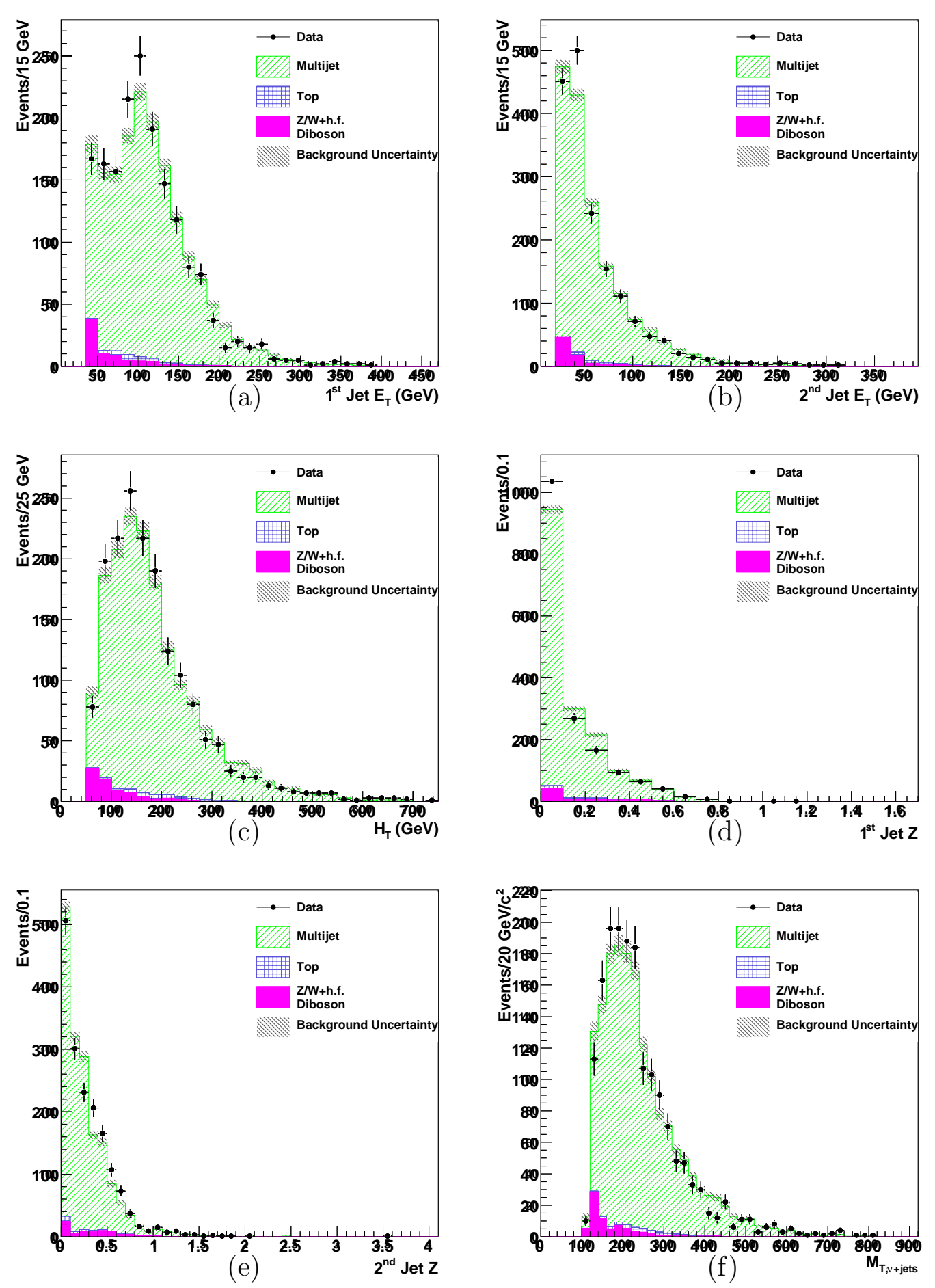

Figure B.7.: Kinematic distributions in CR3 for events with single $b$-tag: (a) $1^{\text {st }}$ jet $E_{T}$, (b) $2^{\text {nd }}$ jet $E_{T}$, (c) event $H_{T}$, (d) $1^{\text {st }}$ jet fragmentation function $Z$, (e) $2^{\text {nd }}$ jet fragmentation function $Z$, (f) invariant transverse mass of all jets and $\not_{T}$ 

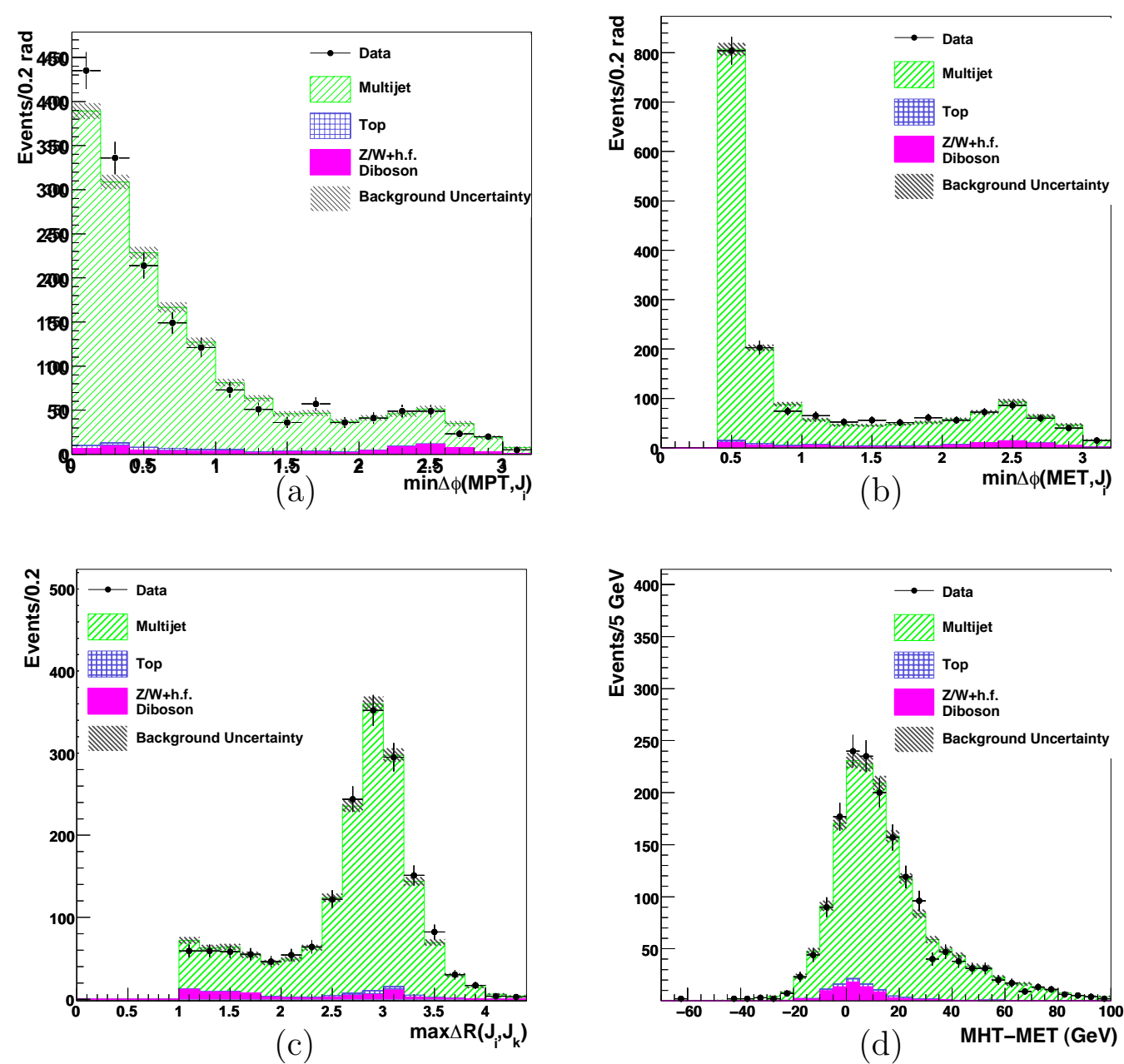

(d)
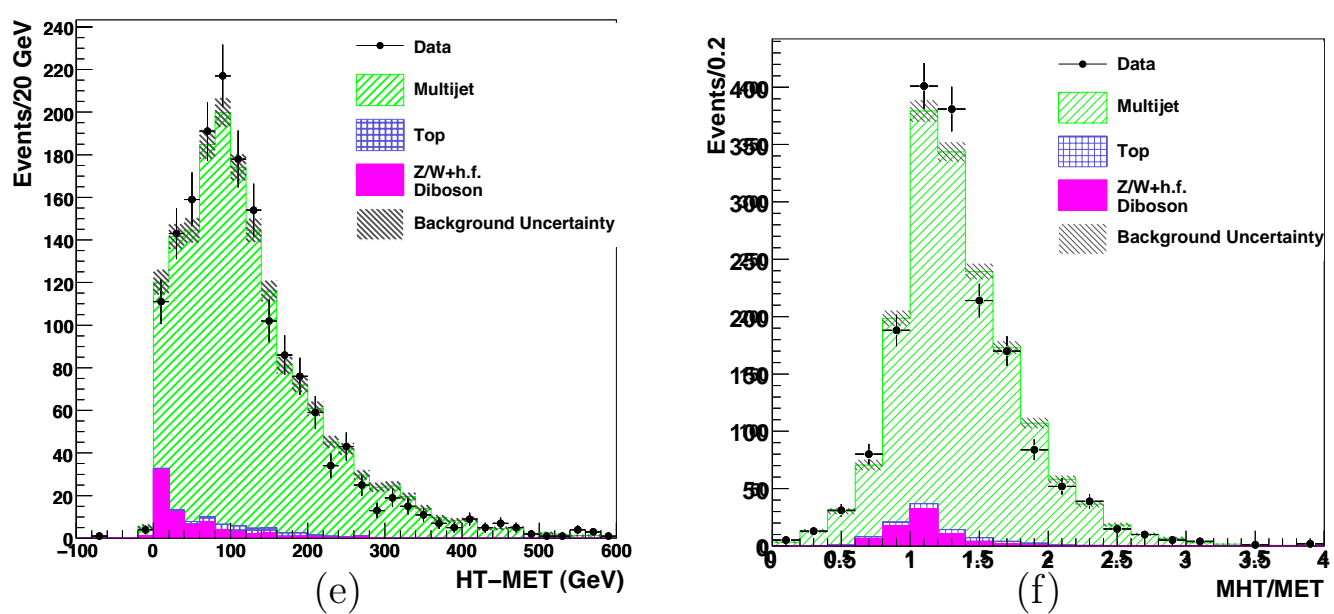

Figure B.8.: Kinematic distributions in CR3 for events with single b-tag: (a) minimum of the difference in $\phi$ between $\overrightarrow{\not P_{T}^{t r}}$ and each jet, (b) minimum of the difference in $\phi$ between $\vec{E}_{T}$ and each jet, (c) maximum of the difference in $R$ space between any two jets, (d) $H_{T}-\#_{T}$, (e) $H_{T}-\notin_{T}$, (f) $\not H_{T} / \notin_{T}$ 

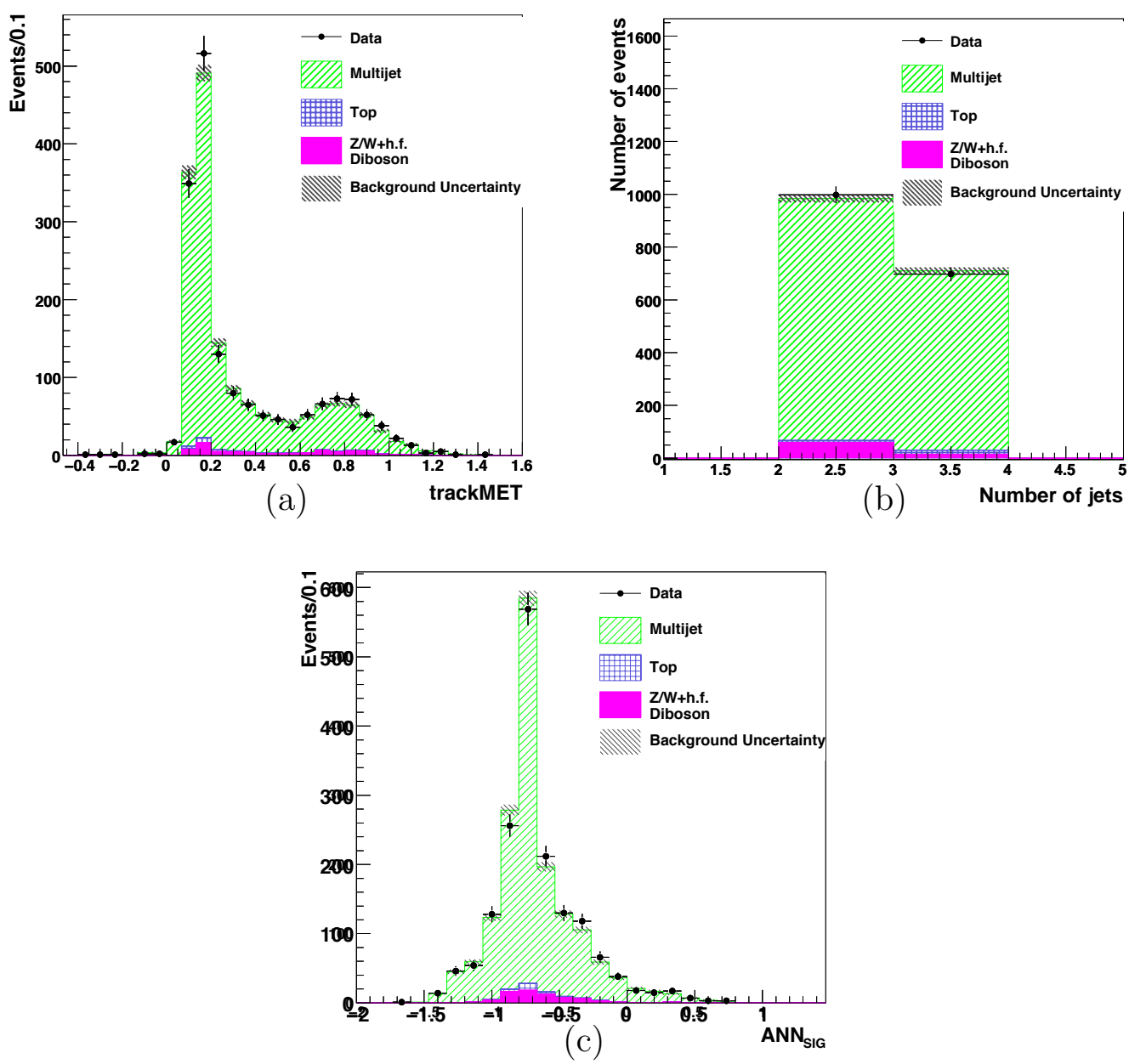

Figure B.9.: Kinematic distributions in CR3 for events with single b-tag: (a) output distribution of $\mathrm{ANN}_{\text {trackMET, }}$, (b) number of jets, (d) output distribution of $\mathrm{ANN}_{\mathrm{SIG}}$ 

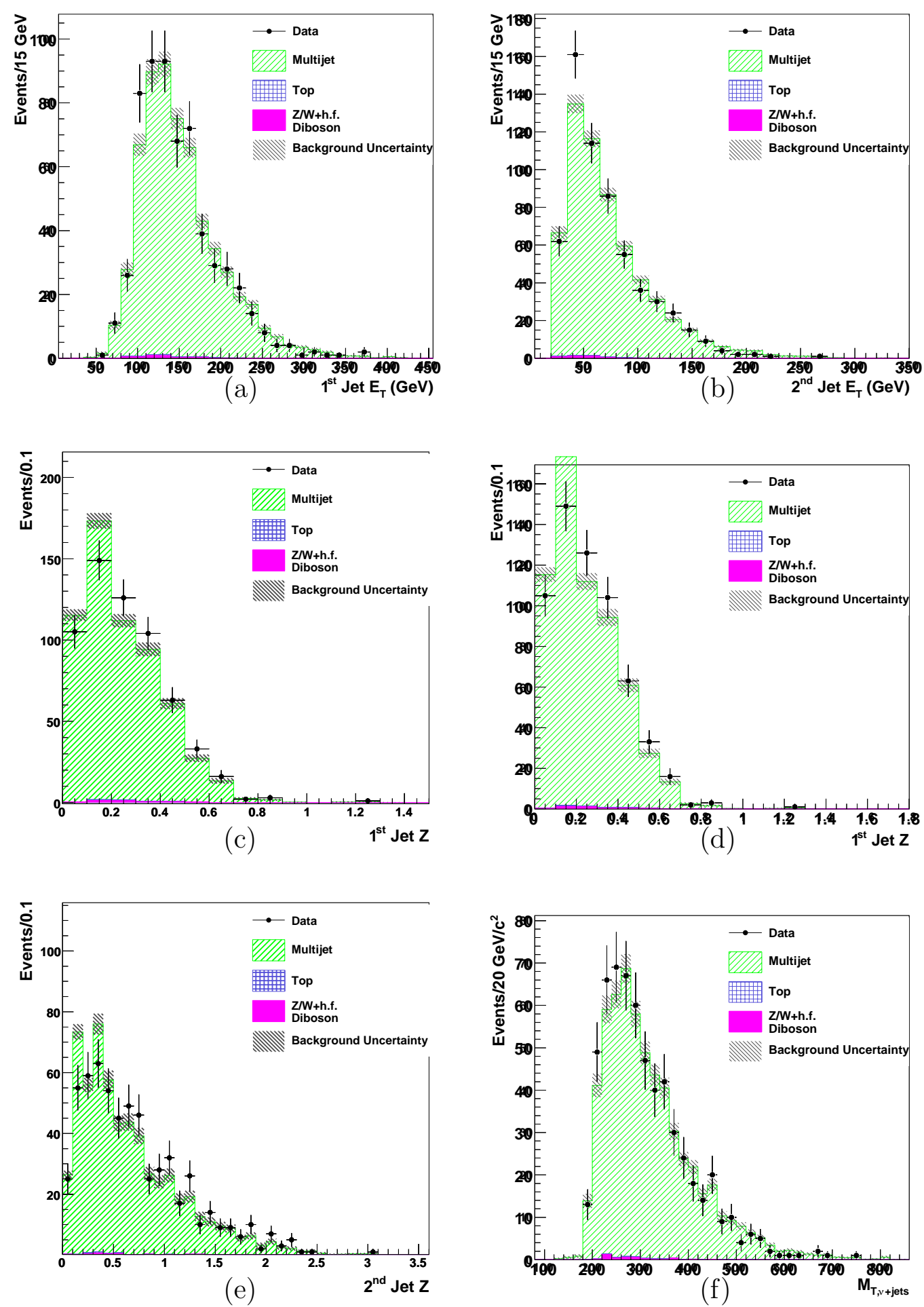

Figure B.10.: Kinematic distributions in CR1 for $\mathrm{ST}+\mathrm{ST}$ events: (a) $1^{\text {st }}$ jet $E_{T}$, (b) $2^{\text {nd }}$ jet $E_{T}$, (c) event $H_{T}$, (d) $1^{\text {st }}$ jet fragmentation function $Z$, (e) $2^{\text {nd }}$ jet fragmentation function $Z$, (f) invariant transverse mass of all jets and $\notin_{T}$ 

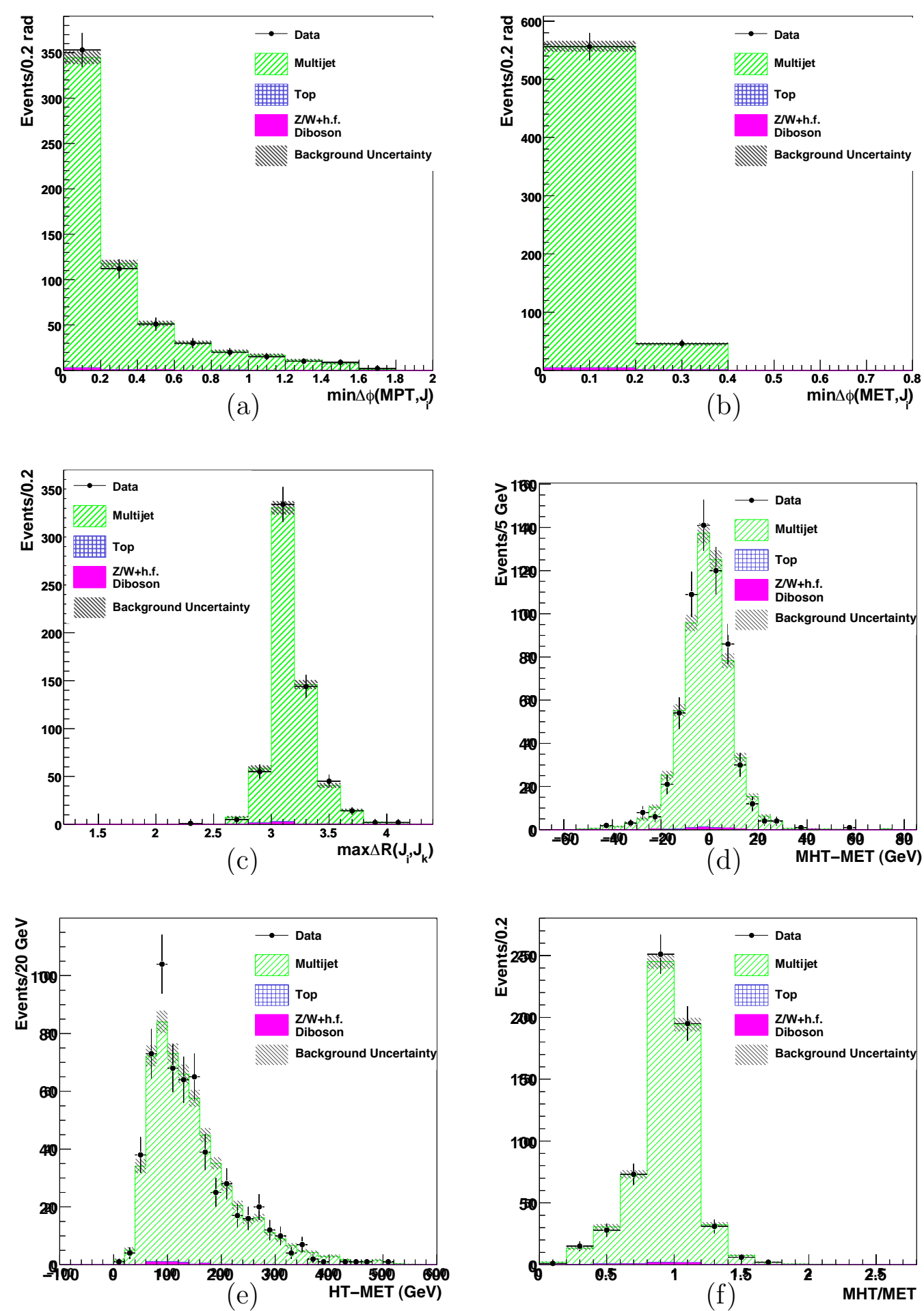

Figure B.11.: Kinematic distributions in CR1 for ST+ST events: (a) minimum of the difference in $\phi$ between $\overrightarrow{\not p_{T}^{t r}}$ and each jet, (b) minimum of the difference in $\phi$ between $\overrightarrow{E_{T}}$ and each jet, (c) maximum of the difference in $R$ space between any two jets, (d) $\not H_{T}-\not_{T}$, (e) $H_{T}-\not_{T}$, (f) $\not H_{T} / \mathscr{E}_{T}$ 

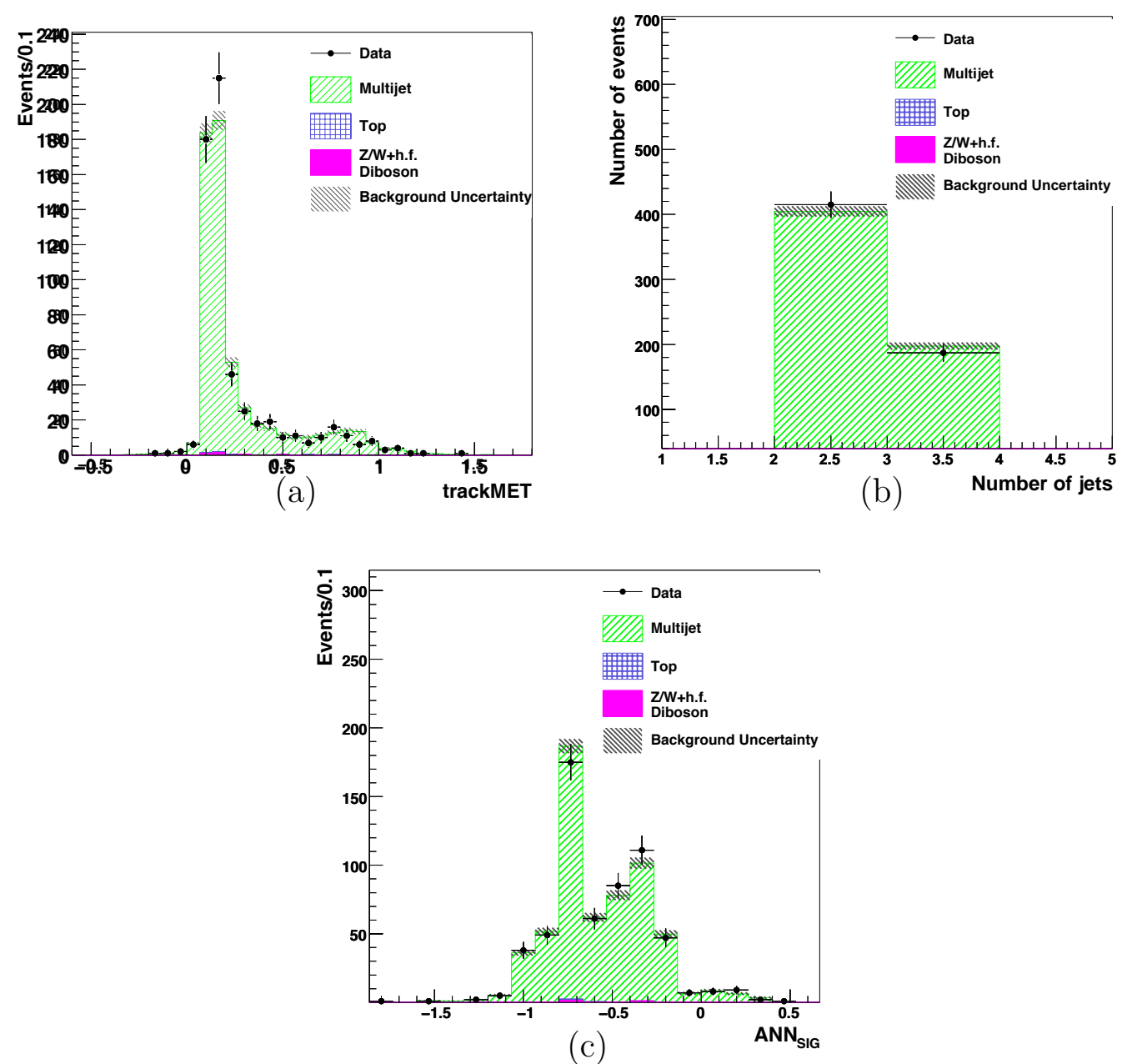

Figure B.12.: Kinematic distributions in CR1 for ST+ST events: (a) output distribution of $\mathrm{ANN}_{\text {trackMET }}$, (b) number of jets, (d) output distribution of $\mathrm{ANN}_{\mathrm{SIG}}$ 

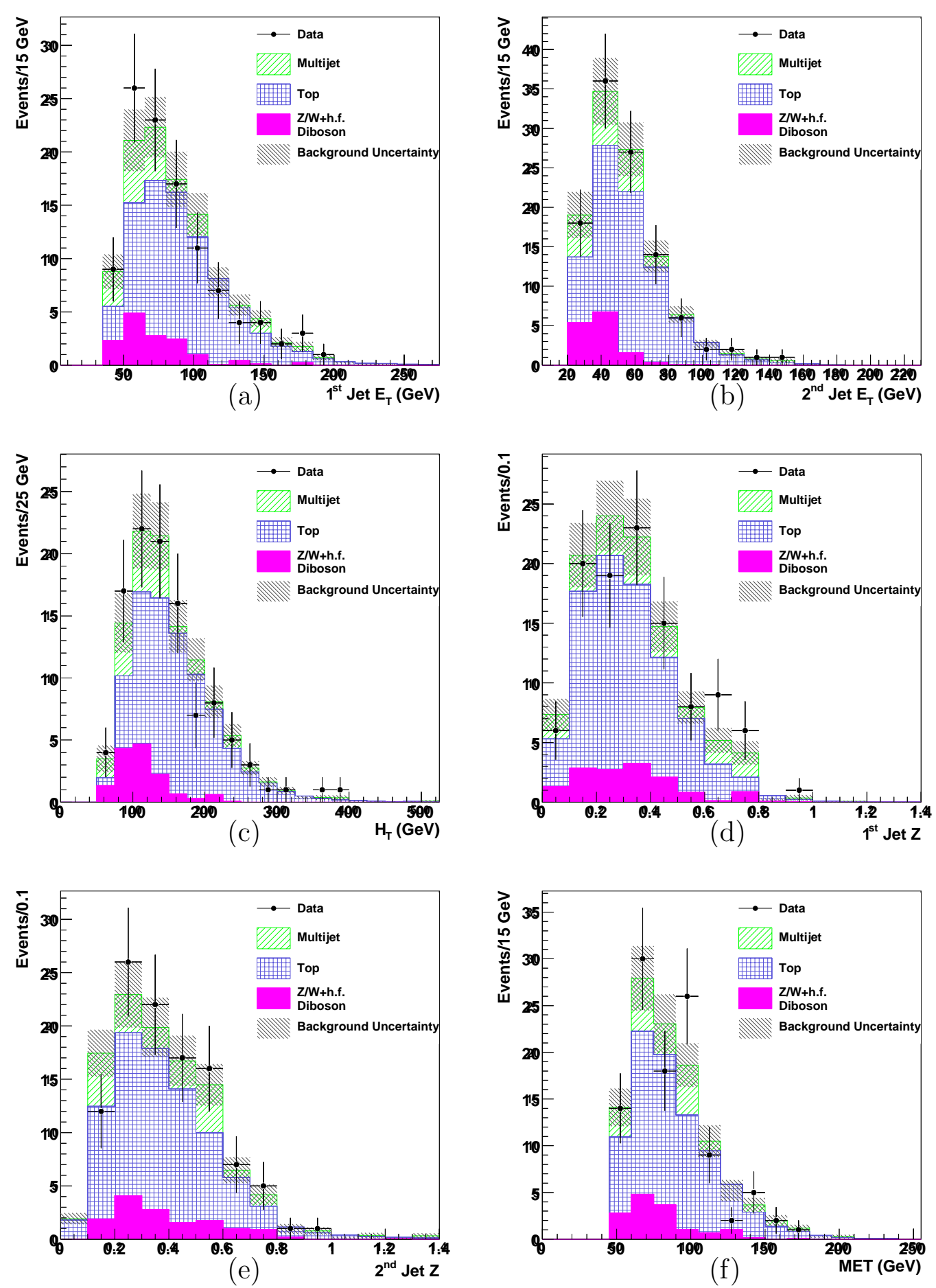

Figure B.13.: Kinematic distributions in CR2 for ST+ST events: (a) $1^{\text {st }}$ jet $E_{T}$, (b) $2^{\text {nd }}$ jet $E_{T}$, (c) event $H_{T}$, (d) $1^{\text {st }}$ jet fragmentation function $Z$, (e) $2^{\text {nd }}$ jet fragmentation function $Z$, (f) event $\notin_{T}$ 


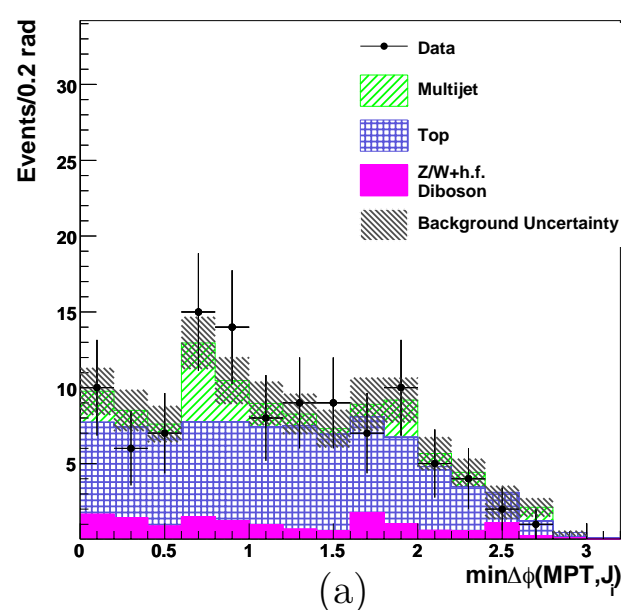

(a)
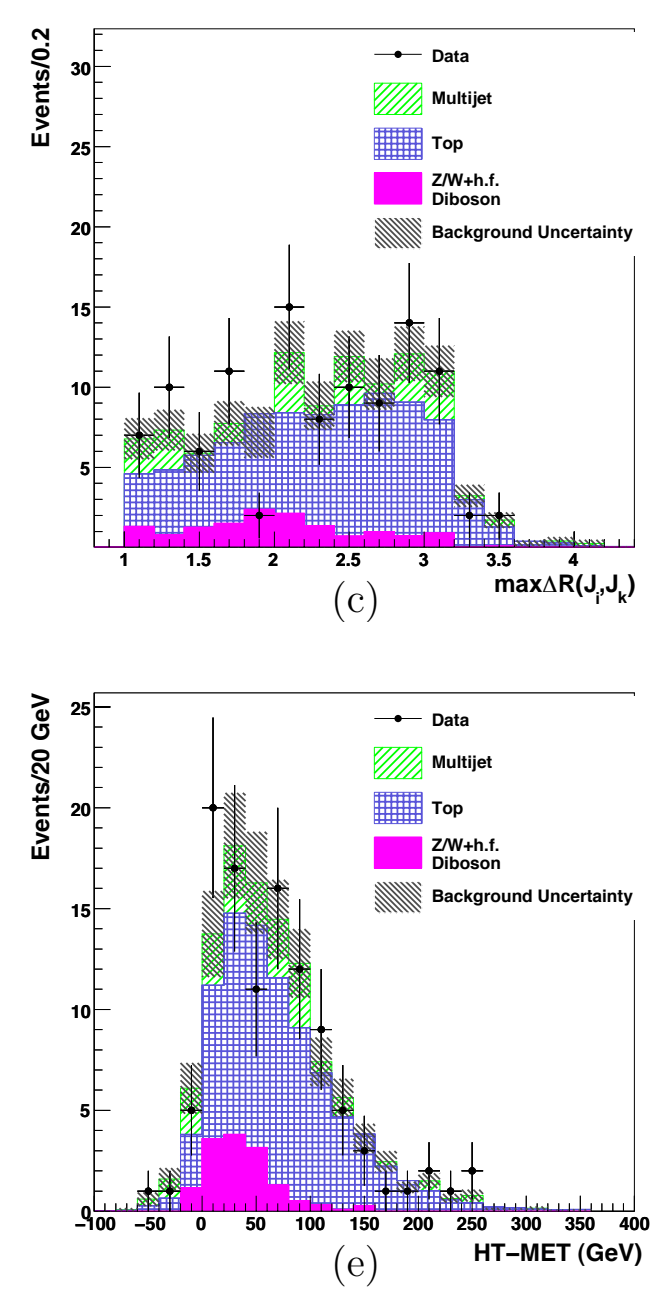
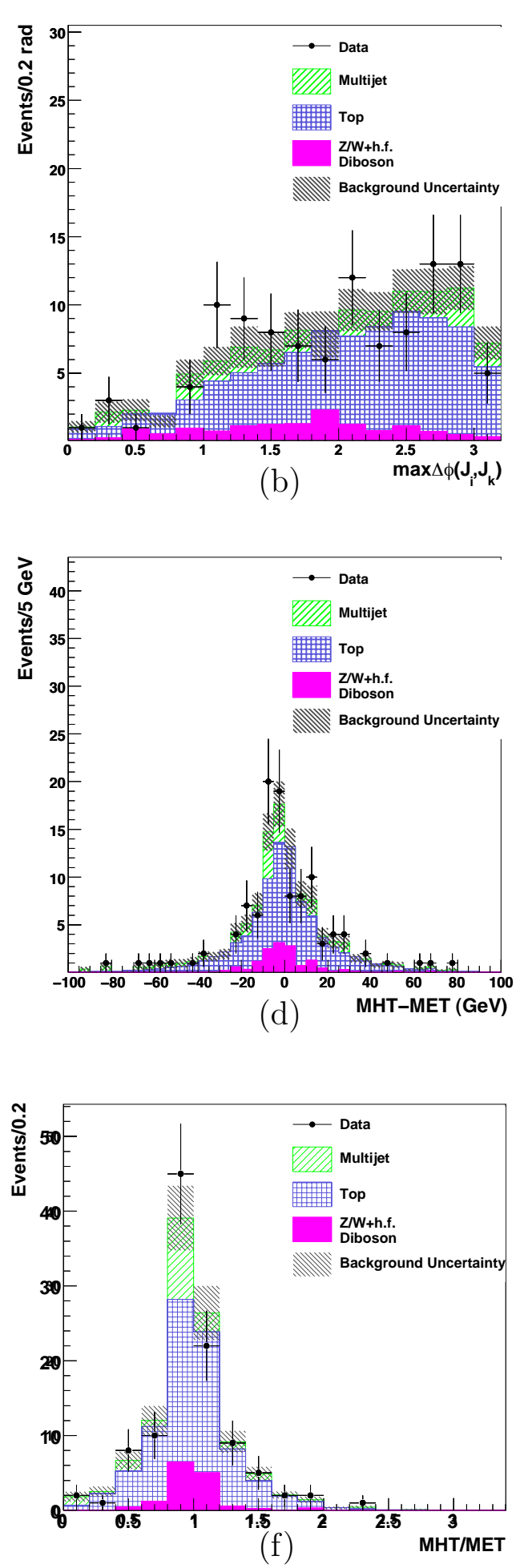

Figure B.14.: Kinematic distributions in CR2 for ST+ST events: (a) minimum of the difference in $\phi$ between $\overrightarrow{P_{T}^{t r}}$ and each jet, (b) maximum of the difference in phi between two jets directions, (c) maximum of the difference in $R$ space between any two jets, (d) $\not_{T}-\not_{T}$, (e) $H_{T}-\mathscr{E}_{T}$, (f) $\not_{T} / \mathscr{E}_{T}$ 

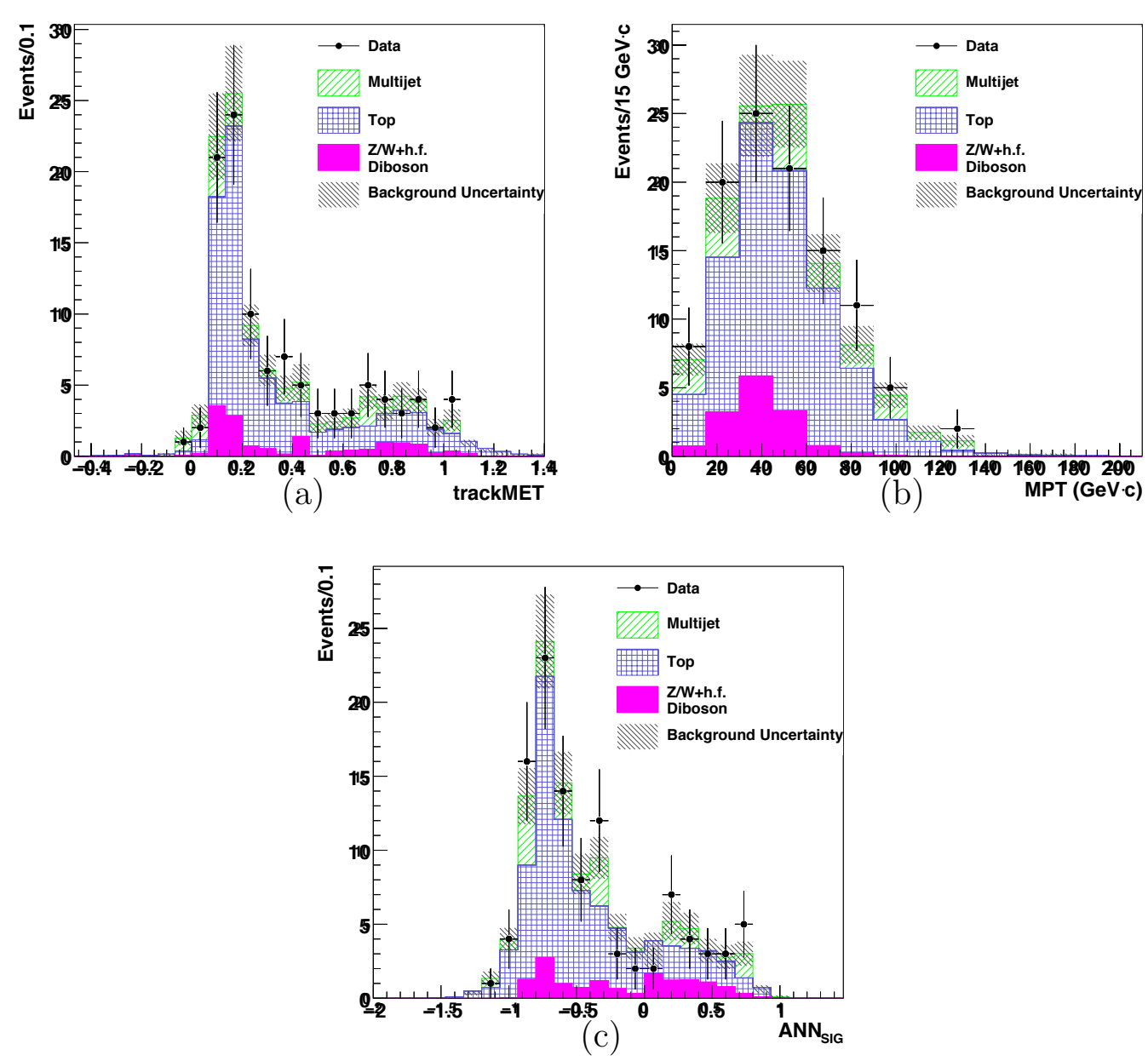

Figure B.15.: Kinematic distributions in CR2 for ST+ST events: (a) output distribution of $\mathrm{ANN}_{\text {trackMET }}$, (b) event $\not P_{T}^{t r}$, (d) output distribution of $\mathrm{ANN}_{\mathrm{SIG}}$ 

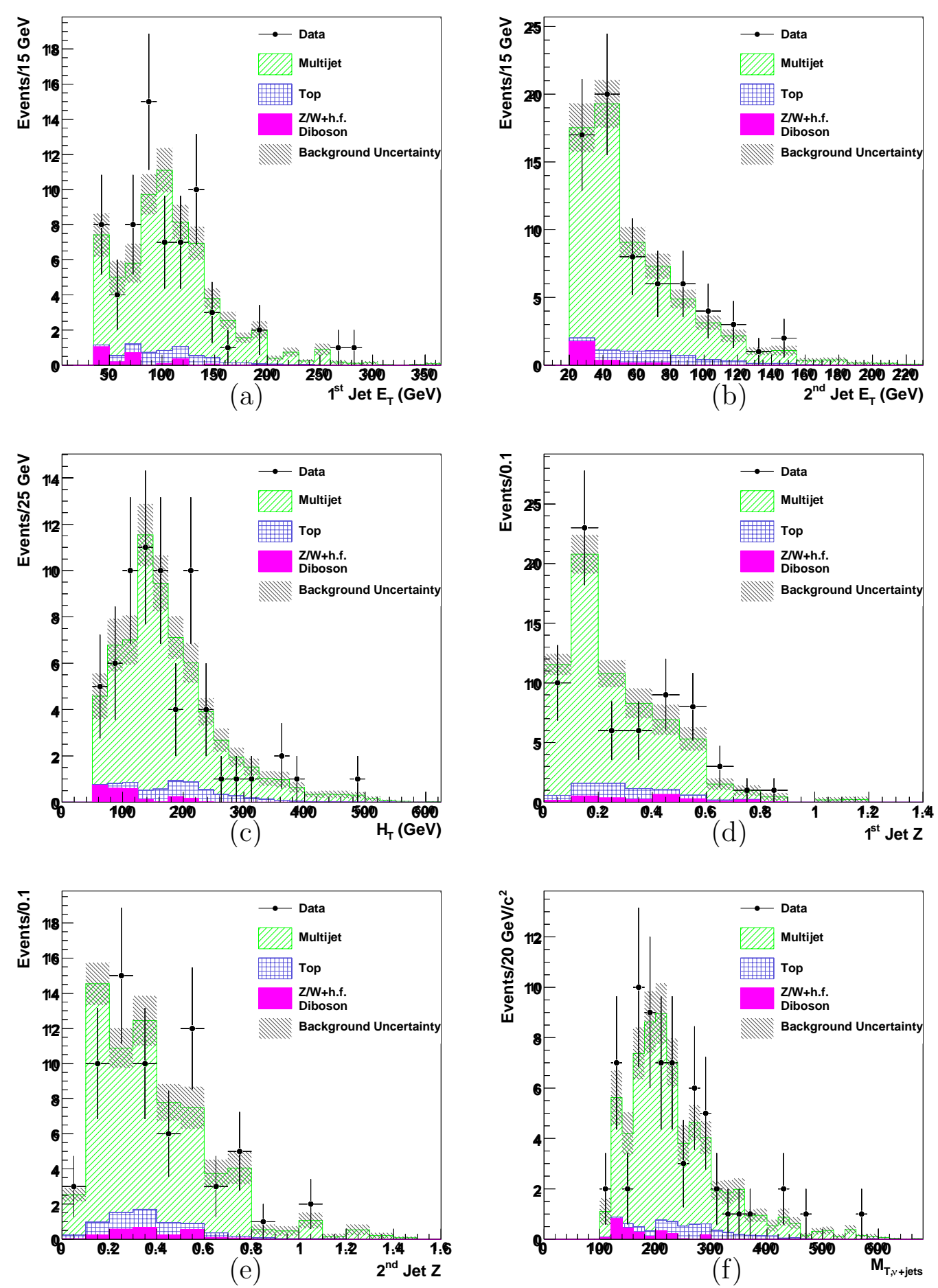

Figure B.16.: Kinematic distributions in CR3 for ST+ST events: (a) $1^{\text {st }}$ jet $E_{T}$, (b) $2^{\text {nd }}$ jet $E_{T}$, (c) event $H_{T}$, (d) $1^{\text {st }}$ jet fragmentation function $Z$, (e) $2^{\text {nd }}$ jet fragmentation function $Z$, (f) invariant transverse mass of all jets and $\notin_{T}$ 

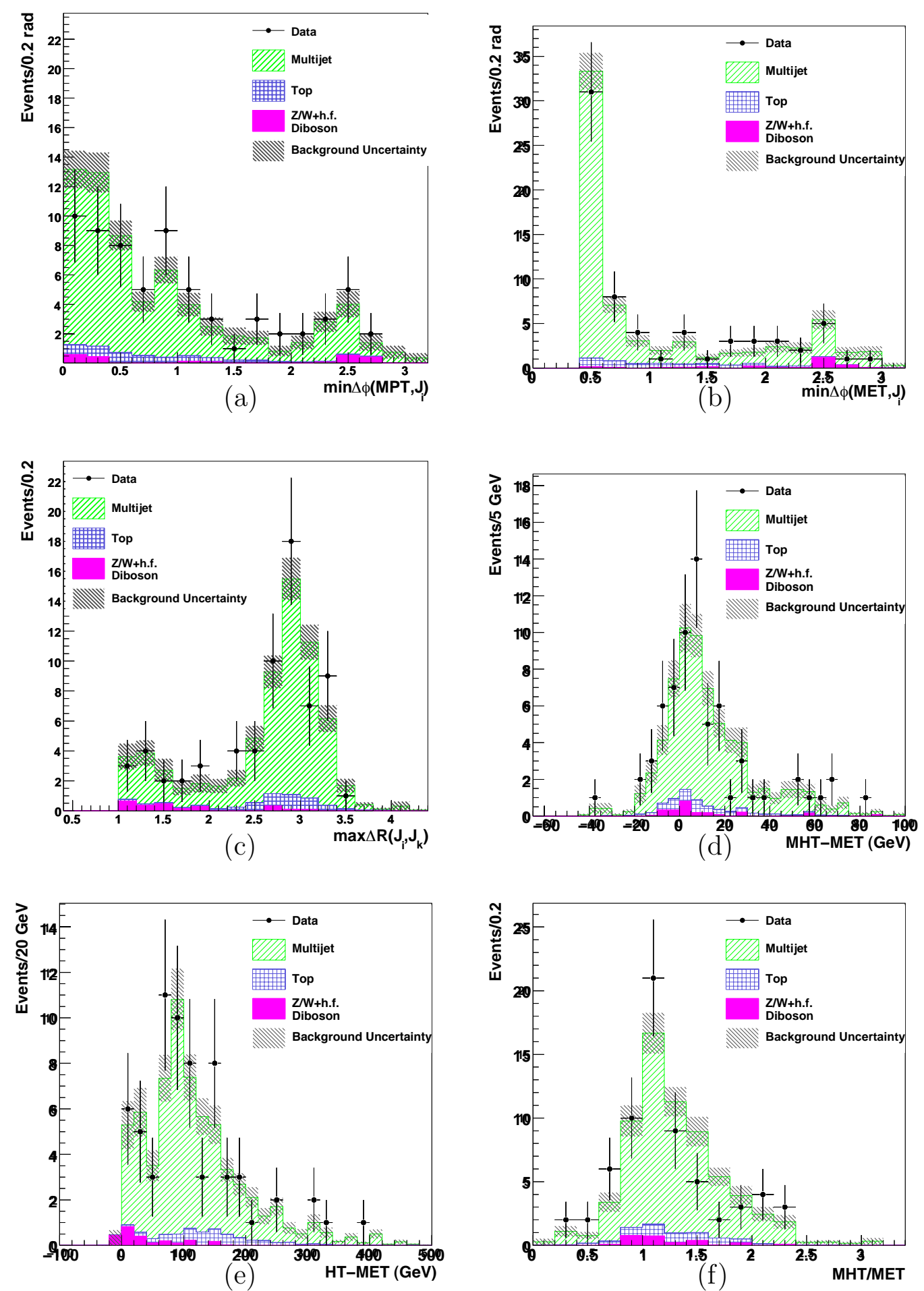

Figure B.17.: Kinematic distributions in CR3 for ST+ST events: (a) minimum of the difference in $\phi$ between $\overrightarrow{\not P_{T}^{t r}}$ and each jet, (b) minimum of the difference in $\phi$ between $\overrightarrow{E_{T}}$ and each jet, (c) maximum of the difference in $R$ space between any two jets, (d) $\not H_{T}-\not_{T}$, (e) $H_{T}-\not_{T}$, (f) $\not H_{T} / \mathscr{E}_{T}$ 

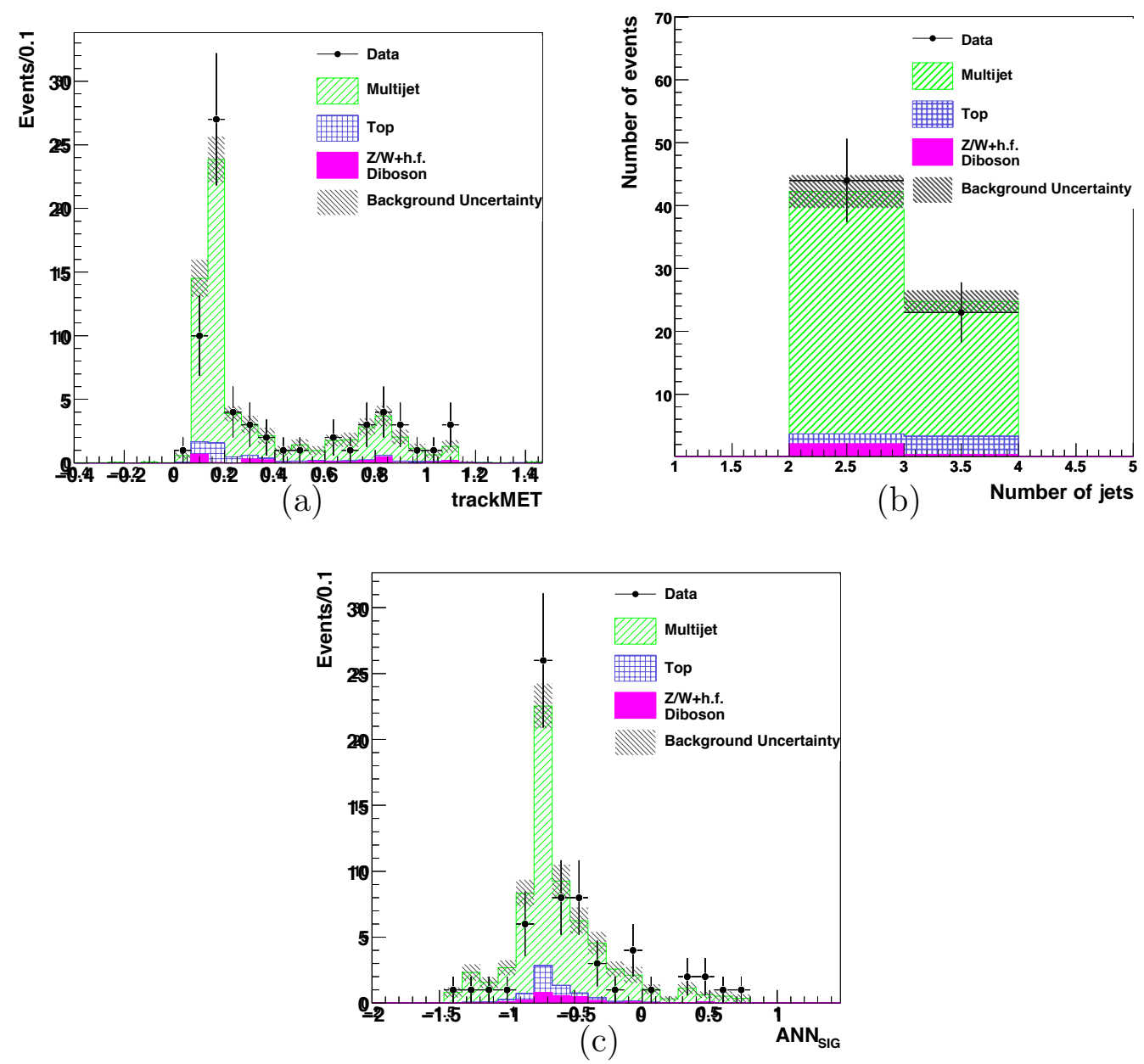

Figure B.18.: Kinematic distributions in CR3 for ST+ST events: (a) output distribution of $\mathrm{ANN}_{\text {trackMET, }}$ (b) number of jets, (d) output distribution of $\mathrm{ANN}_{\mathrm{SIG}}$ 

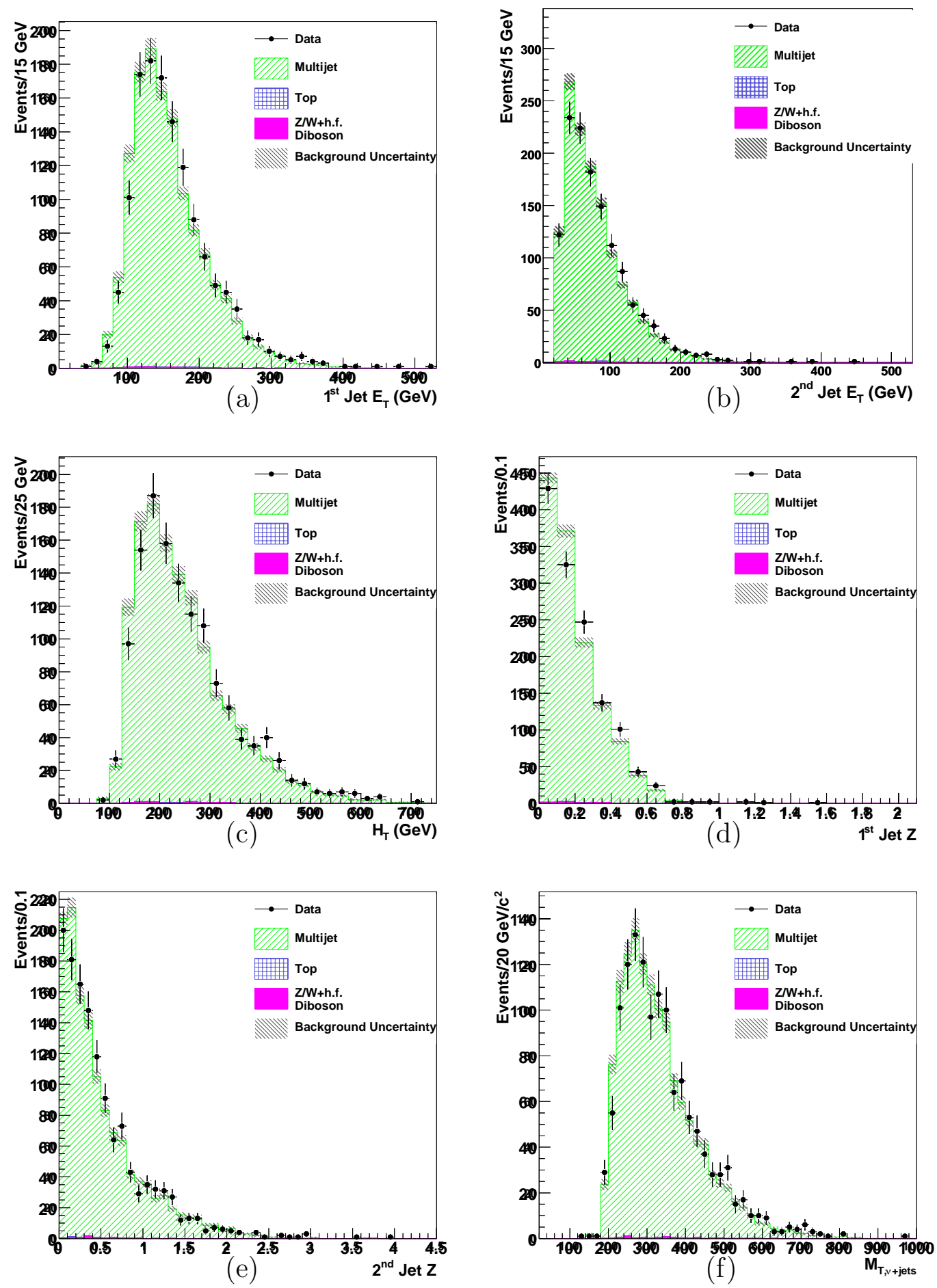

Figure B.19.: Kinematic distributions in CR1 for ST+JP events : (a) $1^{\text {st }}$ jet $E_{T}$, (b) $2^{\text {nd }}$ jet $E_{T}$, (c) event $H_{T}$, (d) $1^{\text {st }}$ jet fragmentation function $Z$, (e) $2^{\text {nd }}$ jet fragmentation function $Z$, (f) invariant transverse mass of all jets and $\notin_{T}$ 

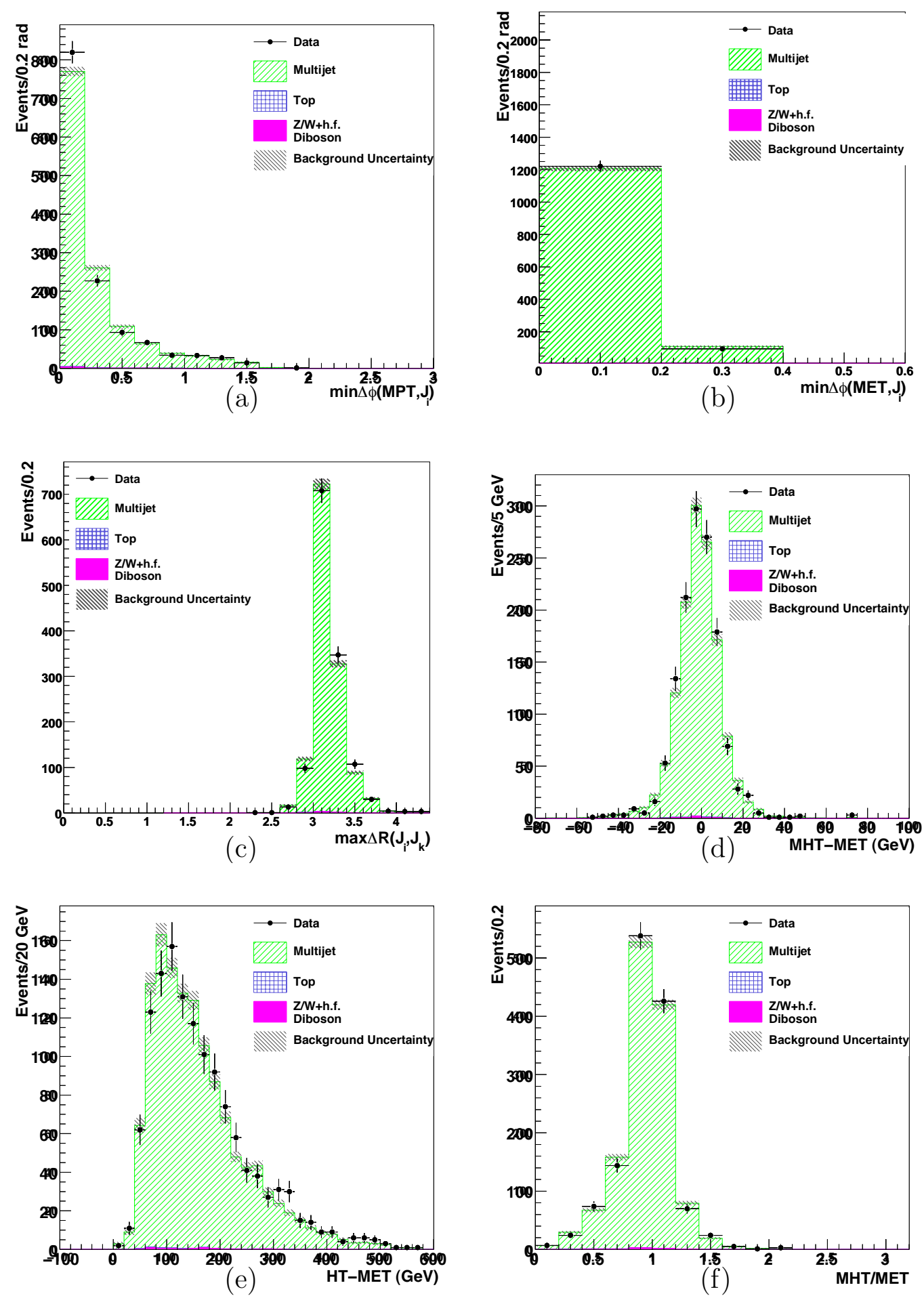

Figure B.20.: Kinematic distributions in CR1 for ST+JP events: (a) minimum of the difference in $\phi$ between $\overrightarrow{\not P_{T}^{t r}}$ and each jet, (b) minimum of the difference in $\phi$ between $\overrightarrow{E_{T}}$ and each jet, (c) maximum of the difference in $R$ space between any two jets, (d) $\not H_{T}-\not_{T}$, (e) $H_{T}-\not_{T}$, (f) $\not H_{T} / \mathscr{E}_{T}$ 

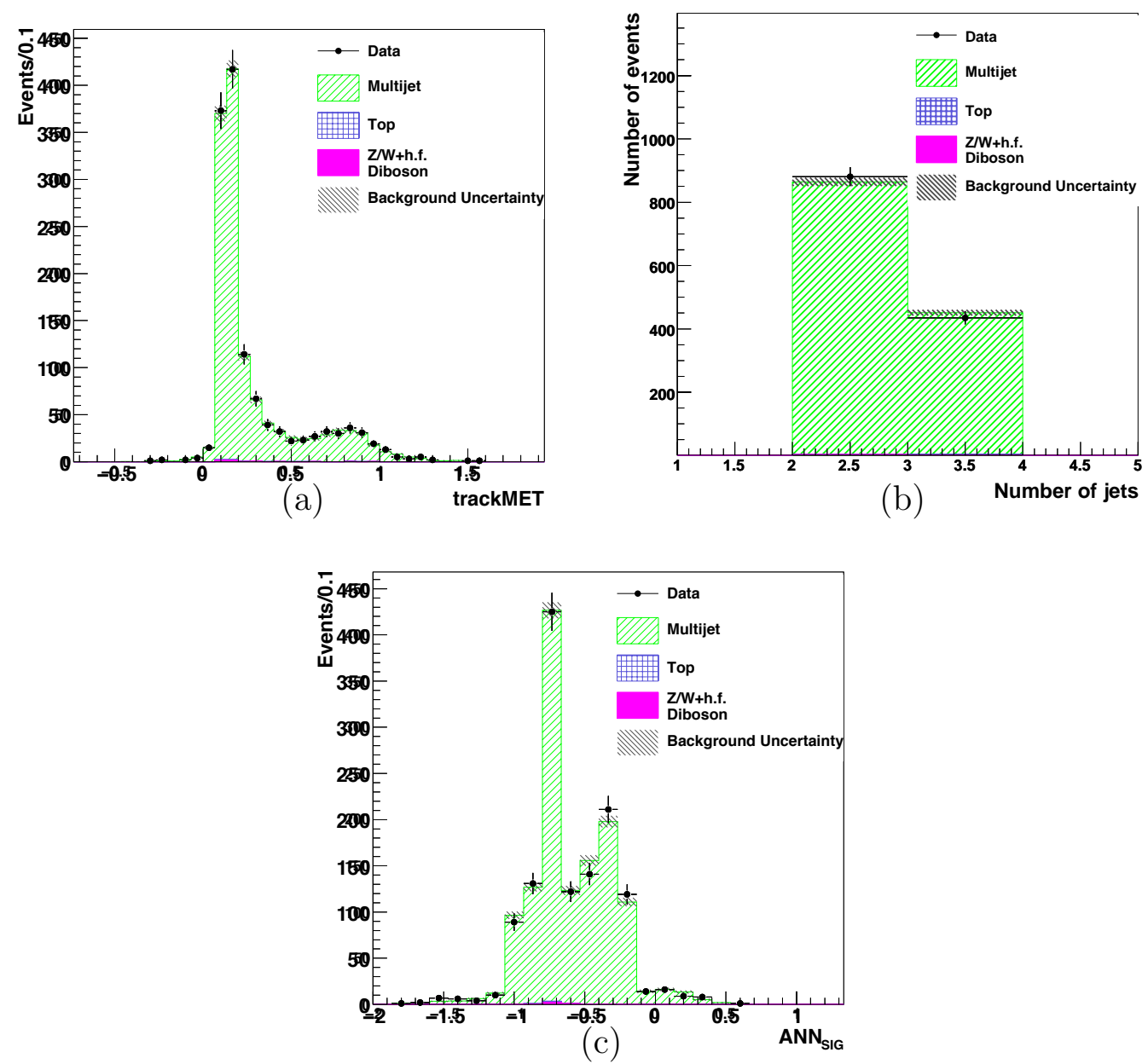

Figure B.21.: Kinematic distributions in CR1 for ST+JP events: (a) output distribution of $\mathrm{ANN}_{\text {trackMET, }}$ (b) number of jets, (d) output distribution of $\mathrm{ANN}_{\mathrm{SIG}}$ 

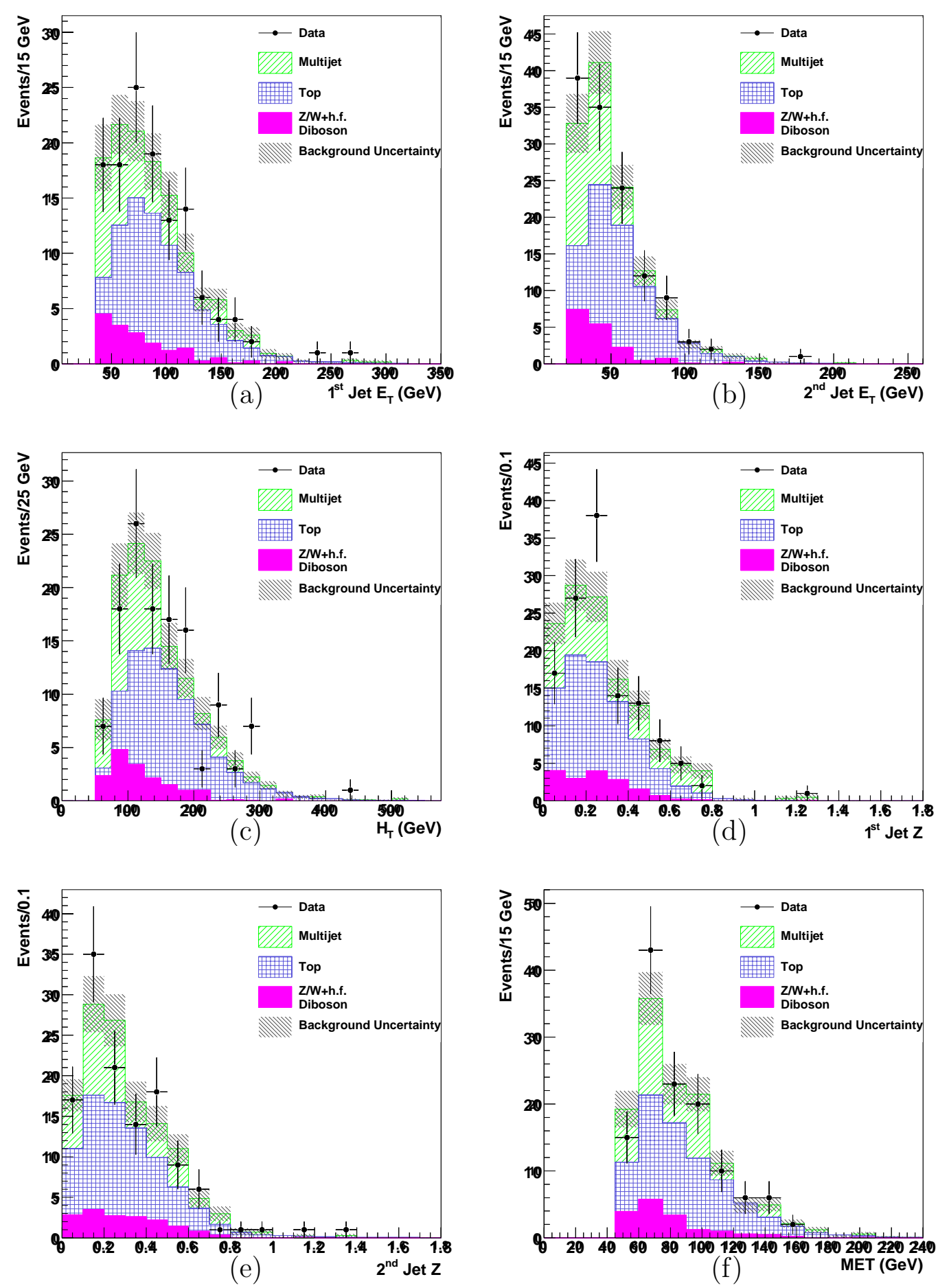

Figure B.22.: Kinematic distributions in CR2 for ST+JP events: (a) $1^{\text {st }}$ jet $E_{T}$, (b) $2^{\text {nd }}$ jet $E_{T},(\mathrm{c})$ event $H_{T}$, (d) $1^{\text {st }}$ jet fragmentation function $Z$, (e) $2^{\text {nd }}$ jet fragmentation function $Z$, (f) event $\notin_{T}$ 


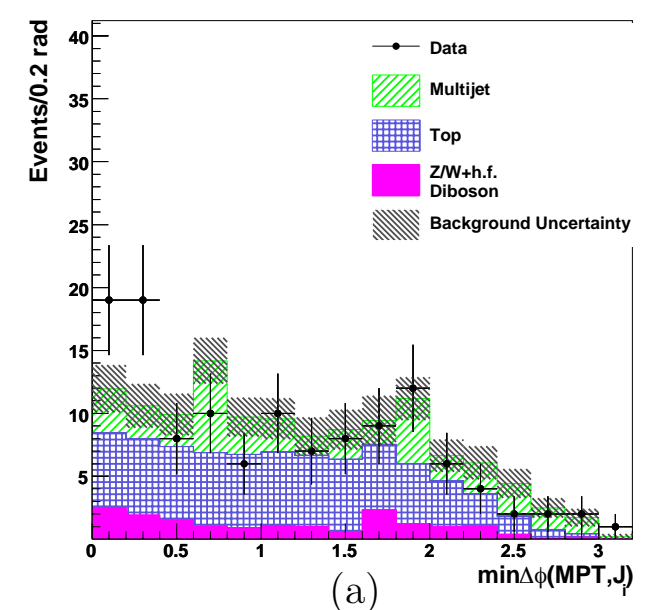

(a)
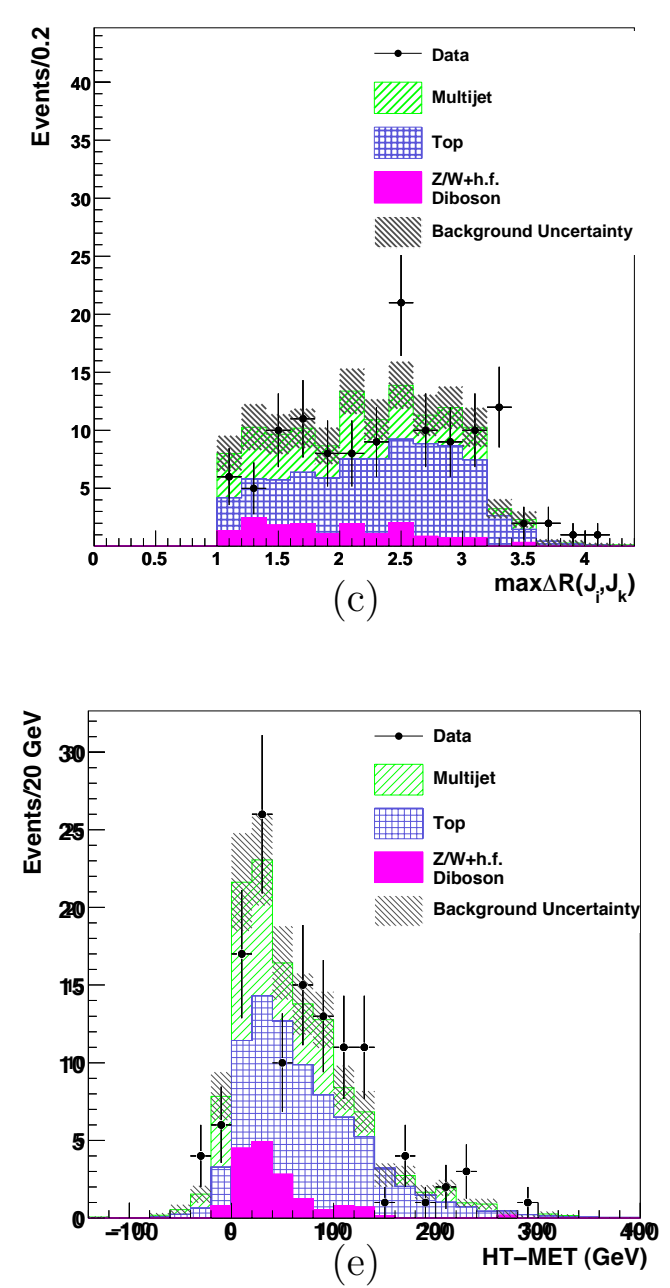
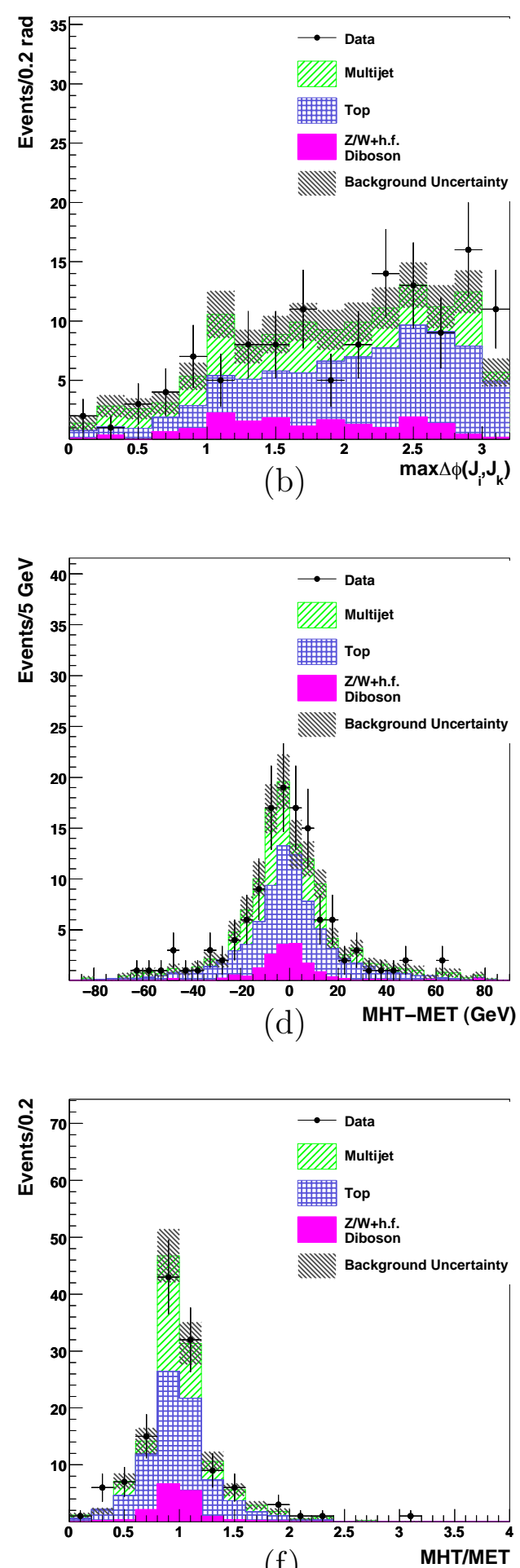

(f)

Figure B.23.: Kinematic distributions in CR2 for ST+JP events: (a) minimum of the difference in $\phi$ between $\overrightarrow{P_{T}^{t r}}$ and each jet, (b) maximum of the difference in phi between two jets directions, (c) maximum of the difference in $R$ space between any two jets, (d) $\not_{T}-\not_{T}$, (e) $H_{T}-\mathscr{E}_{T}$, (f) $\not_{T} / \mathscr{E}_{T}$ 

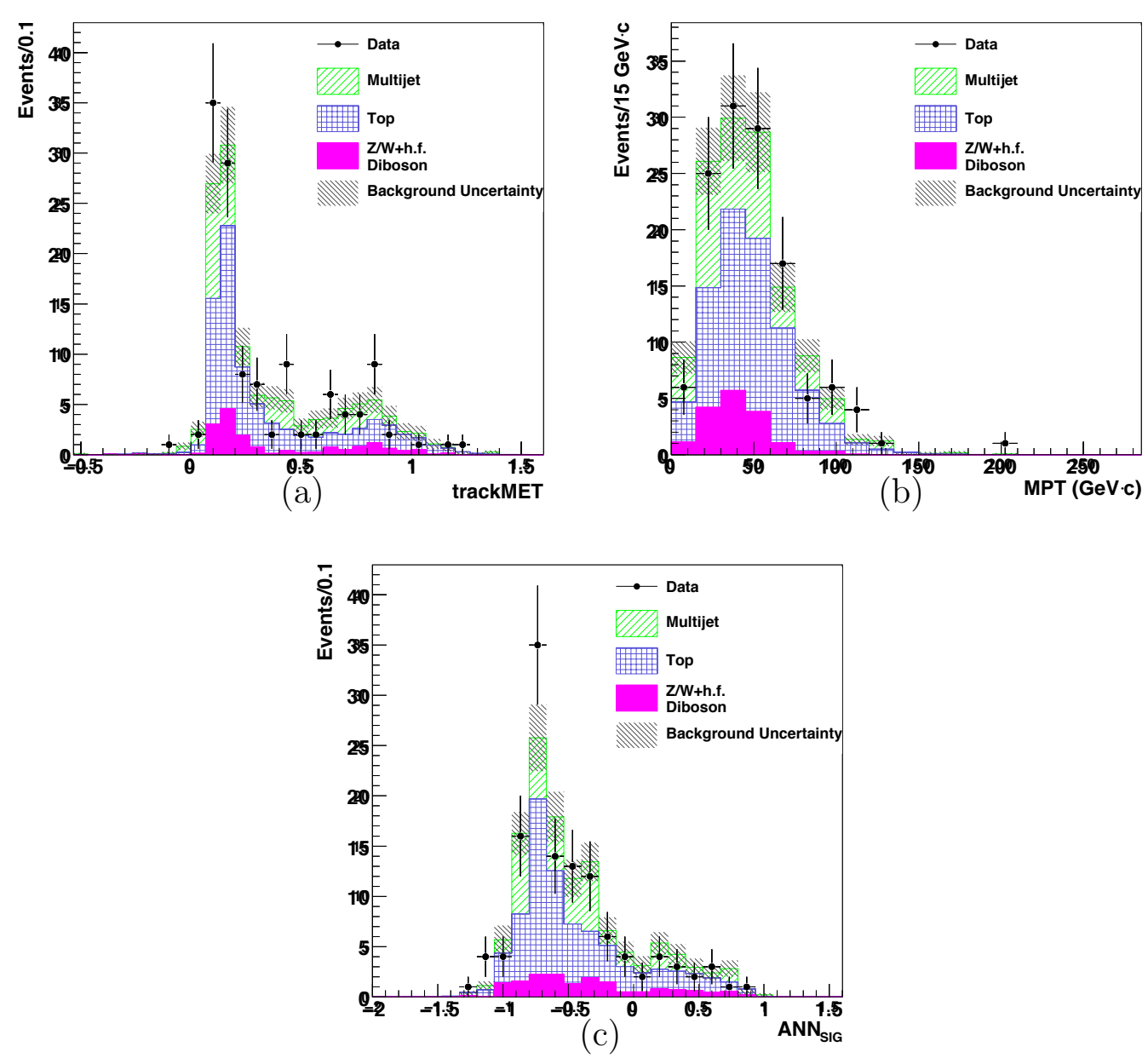

Figure B.24.: Kinematic distributions in CR2 for ST+JP events: (a) output distribution of $\mathrm{ANN}_{\text {trackMET }}$, (b) event $\not P_{T}^{t r}$, (d) output distribution of $\mathrm{ANN}_{\mathrm{SIG}}$ 

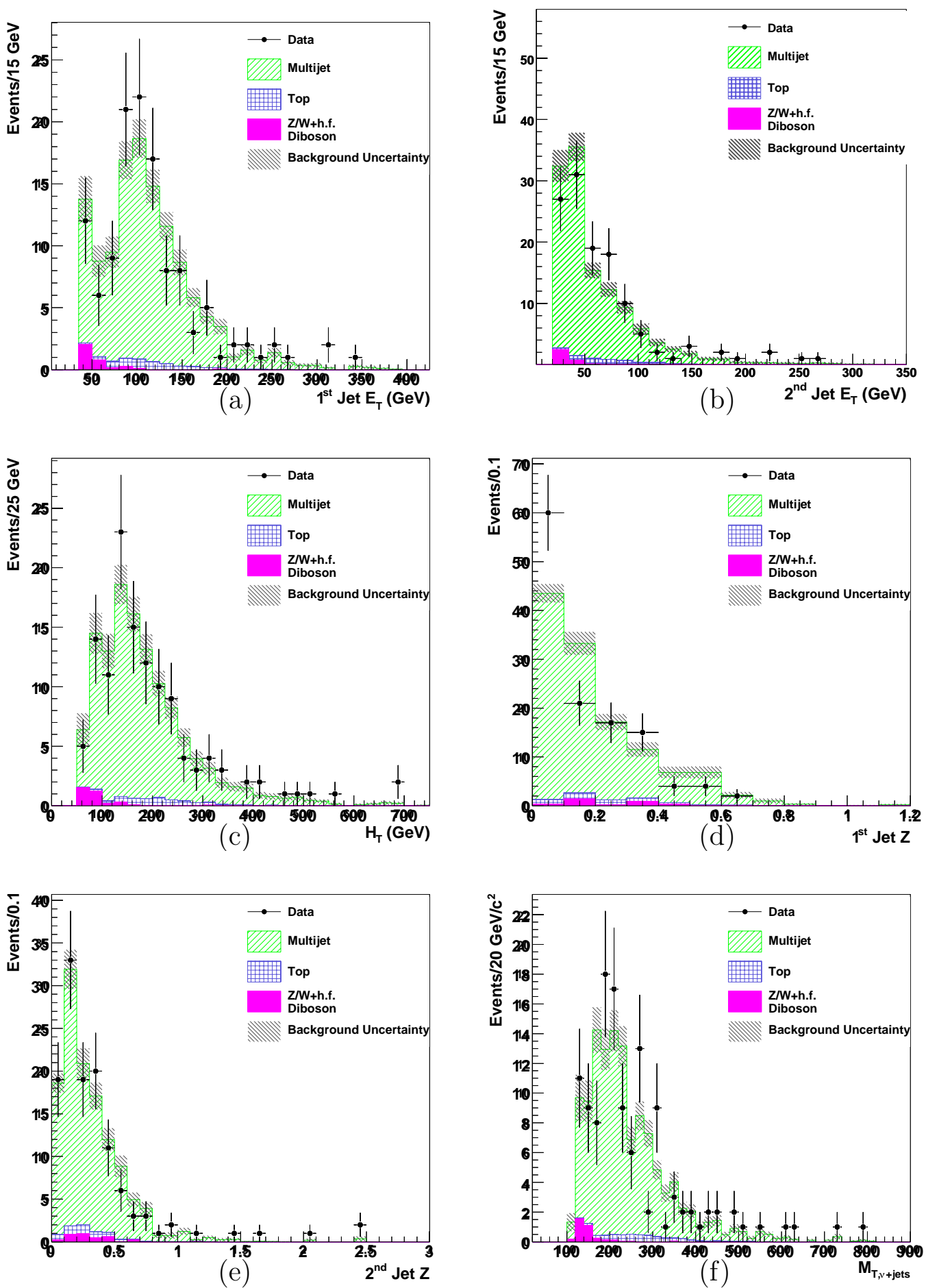

Figure B.25.: Kinematic distributions in CR3 for ST+JP events: (a) $1^{\text {st }}$ jet $E_{T}$, (b) $2^{\text {nd }}$ jet $E_{T}$, (c) event $H_{T}$, (d) $1^{\text {st }}$ jet fragmentation function $Z$, (e) $2^{\text {nd }}$ jet fragmentation function $Z$, (f) invariant transverse mass of all jets and $\notin_{T}$ 

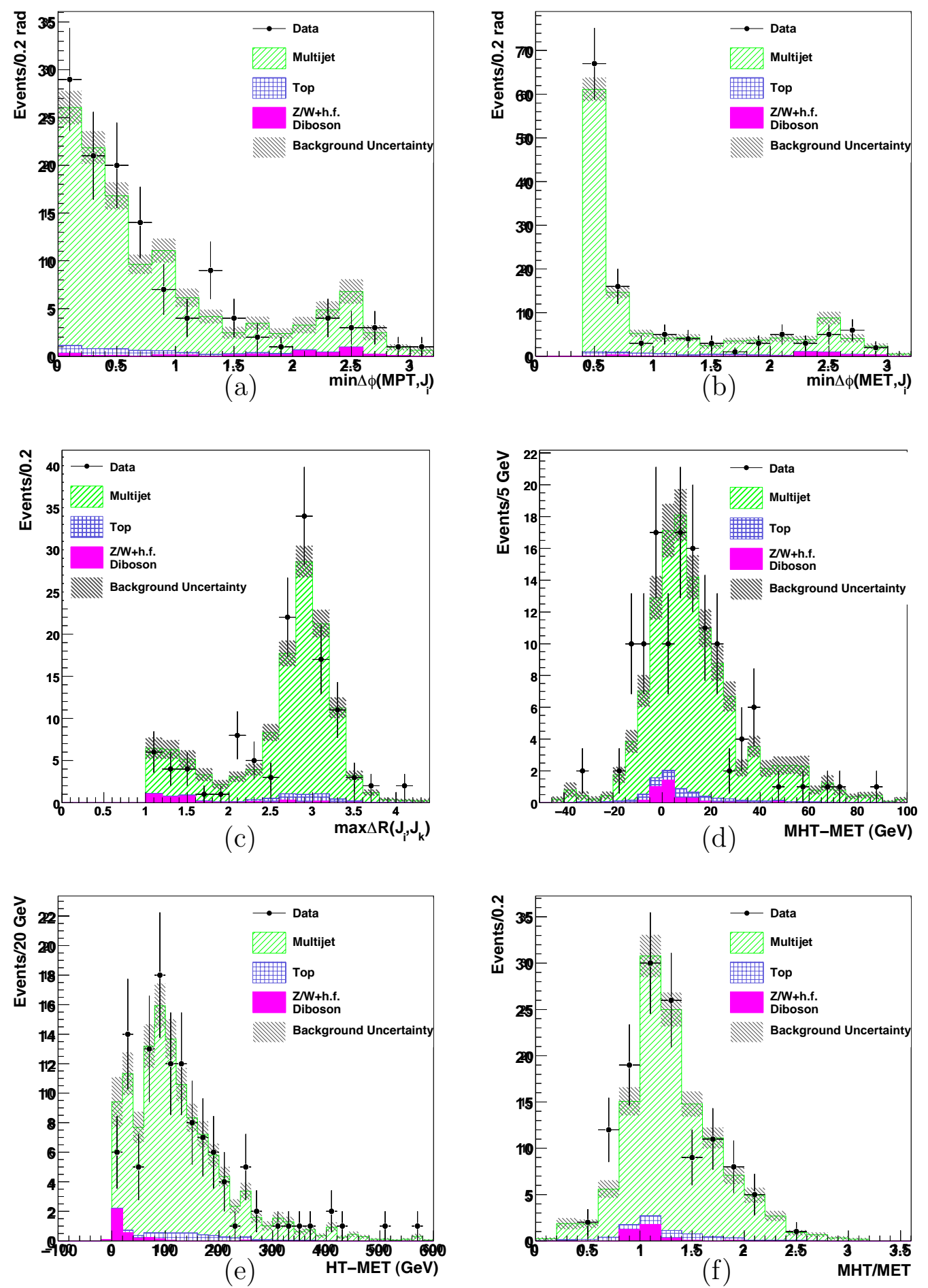

Figure B.26.: Kinematic distributions in CR3 for ST+JP events: (a) minimum of the difference in $\phi$ between $\overrightarrow{\not_{T}^{t r}}$ and each jet, (b) minimum of the difference in $\phi$ between $\overrightarrow{\mathbb{E}_{T}}$ and each jet, (c) maximum of the difference in $R$ space between any two jets, (d) $\not H_{T}-\not_{T}$, (e) $H_{T}-\not_{T}$, (f) $\not H_{T} / \mathscr{E}_{T}$ 

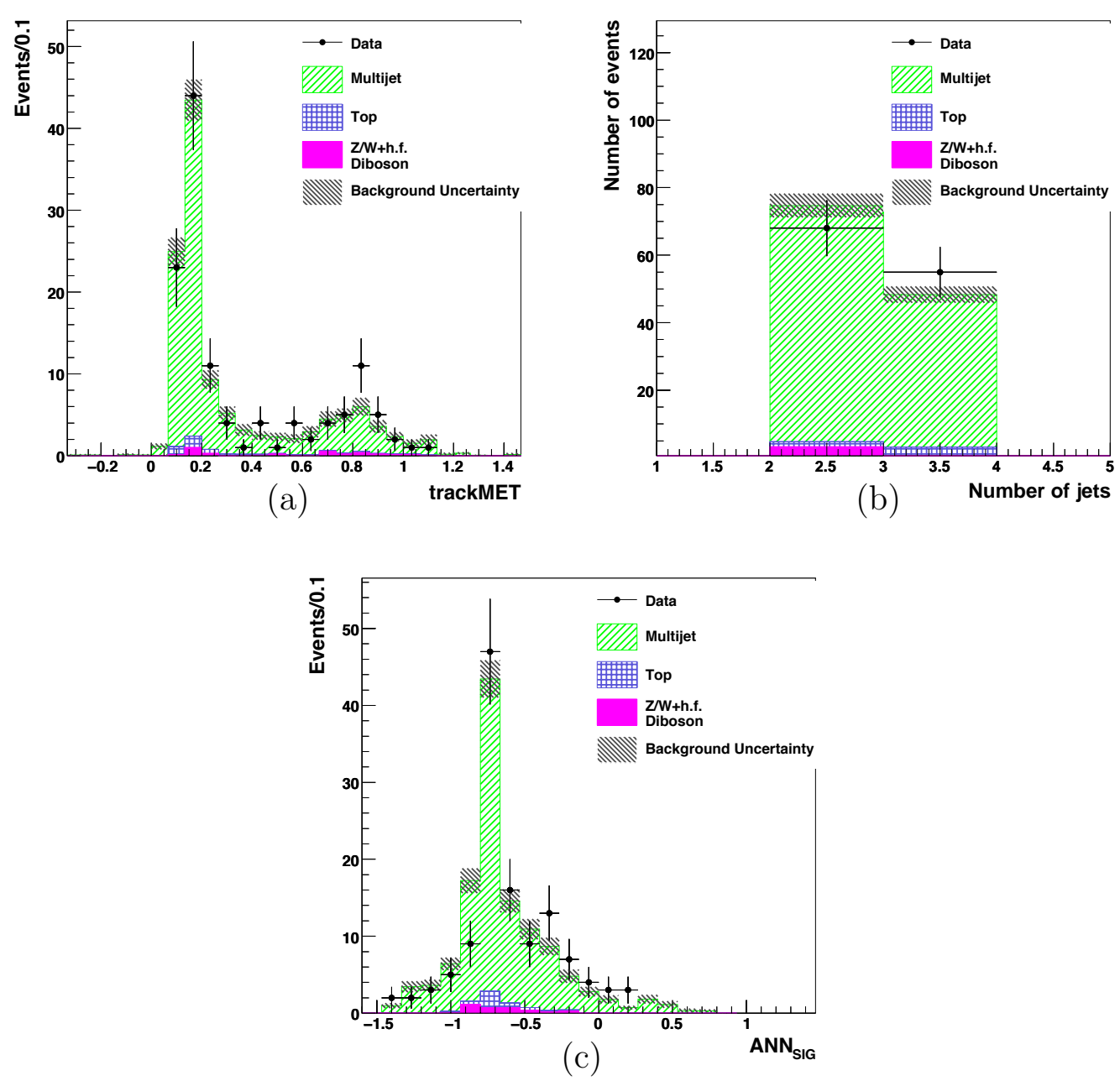

Figure B.27.: Kinematic distributions in CR3 for ST+JP events: (a) output distribution of $\mathrm{ANN}_{\text {trackMET }}$, (b) number of jets, (d) output distribution of $\mathrm{ANN}_{\mathrm{SIG}}$ 


\section{Appendix C: Correlations in Control Regions}

In order to achieve greater sensitivity, we use two ANN's in this analysis. ANN's allow the use of more information available in the events compared to the traditional cut-based event selection. However, careful checks need to be made, to make sure that all of the inputs to the ANN, as well as the correlations between them, are well modelled. Otherwise, the output of the ANN may be biased.

In this chapter we show distributions of correlation coefficients (Eq. 6.1) in control regions. The comparisons are shown for some of the variables that are used as inputs to $\mathrm{ANN}_{\mathrm{MJ}}$ and $\mathrm{ANN}_{\mathrm{SIG}}$. Additional comparisons are available in [101]. 
MinDPhiMET_Ji_vs_mpt, CR1, Exclusive ST

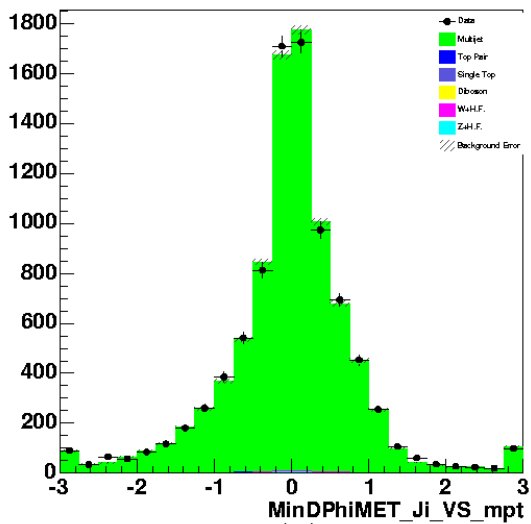

(a)

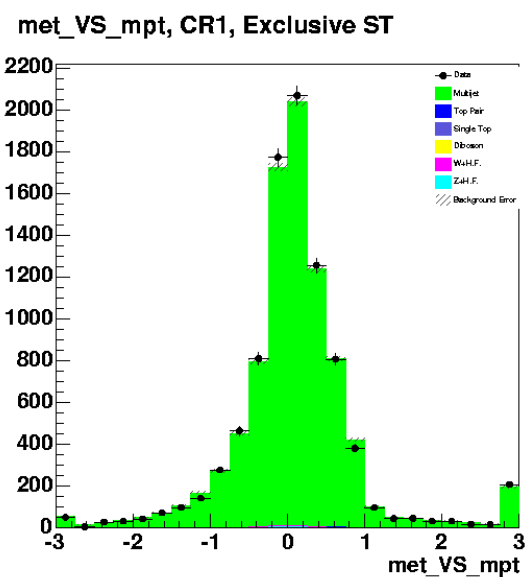

(c)

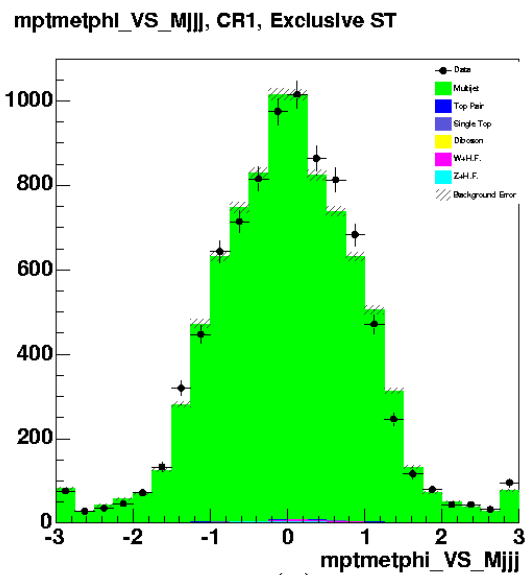

(e)
met_vS_MaxDPhIJs, CR1, Exclusive ST

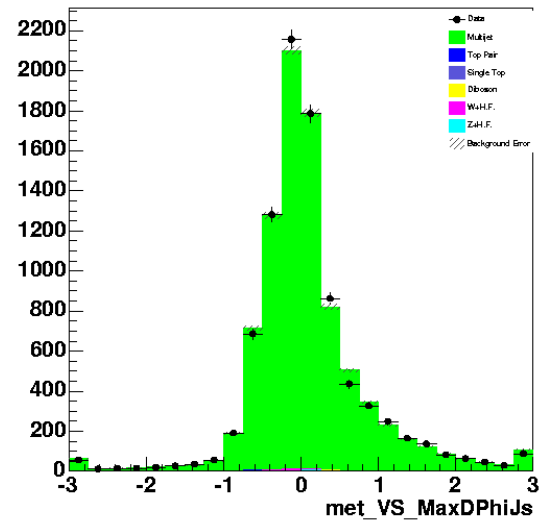

(b)

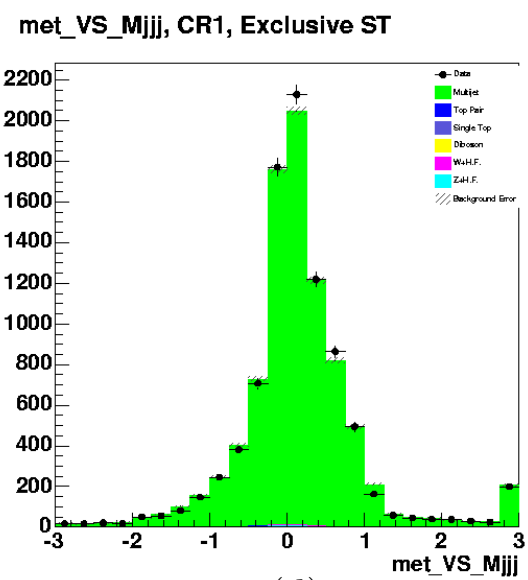

(d)

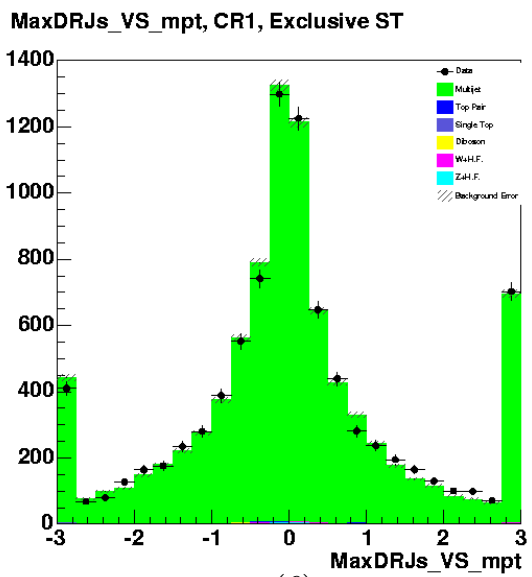

(f)

Figure C.1.: Correlations between some of the important variables in CR1, Exclusive ST events: (a) $r\left(\min \left(\Delta \phi\left(\vec{E}_{T}, \vec{J}_{i}\right)\right), \not \not_{T}^{t r}\right),(\mathrm{b}) r\left(\max \left(\Delta \phi\left(\vec{J}_{i}, \vec{J}_{k}\right)\right), \notin_{T}\right)$, (c) $r\left(\not_{T}, \not_{T}^{t r}\right)$, (d) $r\left(\mathscr{E}_{T}, M_{j j}\right)$, (e) $r\left(\Delta \phi\left(\overrightarrow{\mathscr{E}_{T}}, \overrightarrow{\not_{T}^{t r}}\right), M_{j j}\right)$, (f) $r\left(\max \left(\Delta R\left(\vec{J}_{i}, \vec{J}_{k}\right)\right), \not_{T}^{t r}\right)$. 


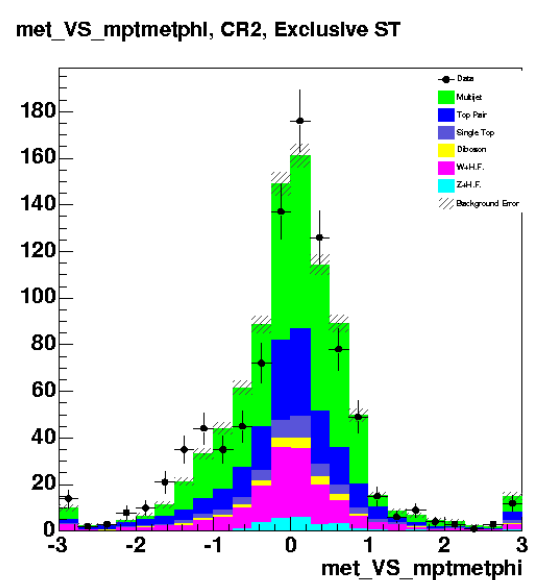

(a)

met_vs_mpt, CR2, Exclusive ST

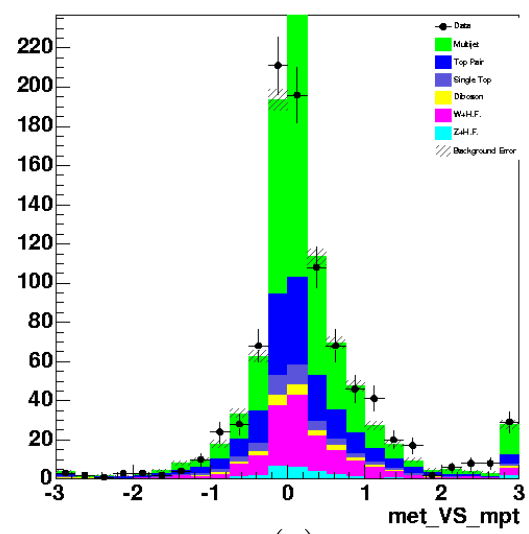

(c)

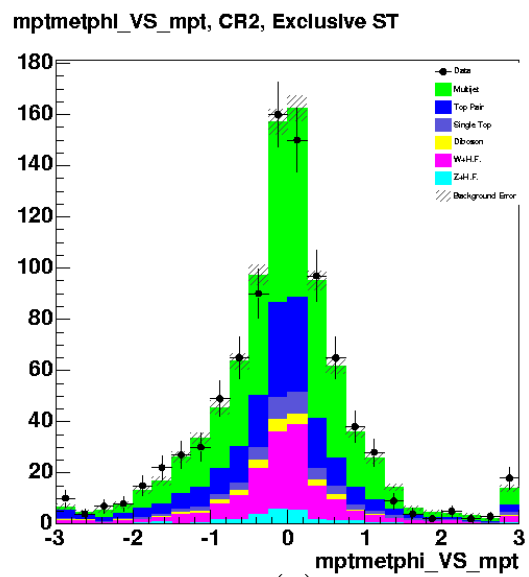

(e)
MinDPhiMET_Ji_vs_Mjjj, CR2, Exclusive ST

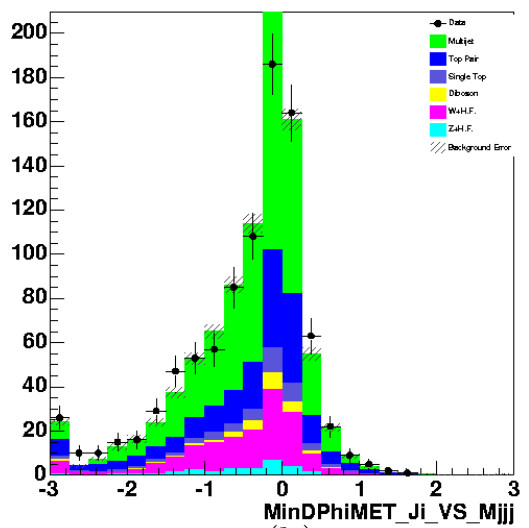

(b)

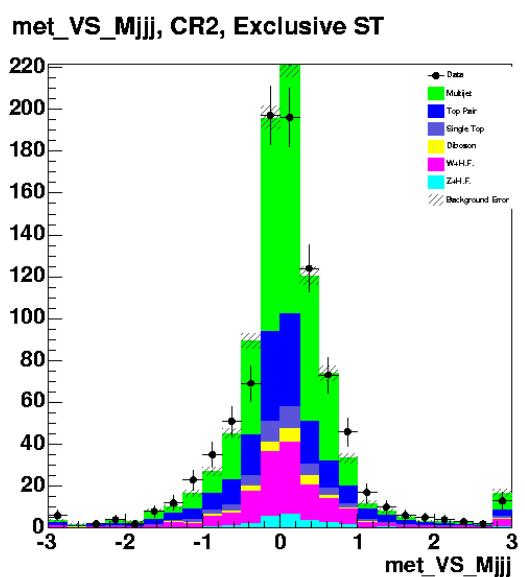

(d)

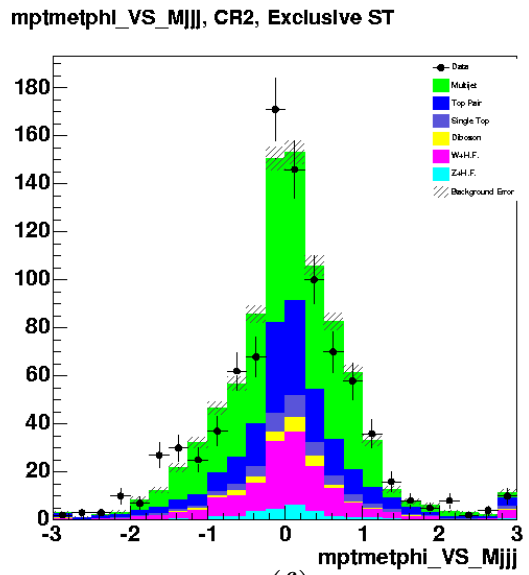

(f)

Figure C.2.: Correlations between some of the important variables in CR2, Exclusive ST events: (a) $r\left(\Delta \phi\left(\overrightarrow{\mathbb{E}_{T}}, \overrightarrow{P_{T}^{t r}}\right), \mathbb{E}_{T}\right)$, (b) $\min \left(\Delta \phi\left(\overrightarrow{\mathscr{E}_{T}}, \overrightarrow{J_{i}}\right), M_{j j}\right)$, (c) $r\left(\mathscr{E}_{T},\left.\not\right|_{T} ^{t r}\right)$, (d) $r\left(\mathscr{E}_{T}, M_{j j}\right),(\mathrm{e}) r\left(\Delta \phi\left(\overrightarrow{\mathbb{E}_{T}}, \overrightarrow{\not_{T}^{t r}}\right), \not_{T}^{t r}\right)$, (f) $r\left(\Delta \phi\left(\overrightarrow{\mathbb{E}_{T}}, \overrightarrow{\phi_{T}^{t r}}\right), M_{j j}\right)$. 
MinDPhiMET_Ji_vS_mpt, CR1, ST+ST

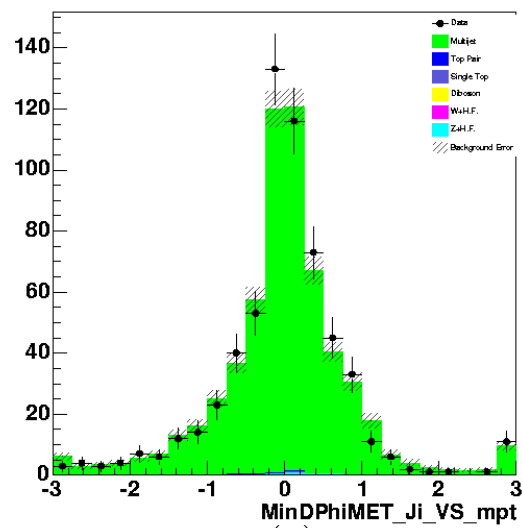

(a)

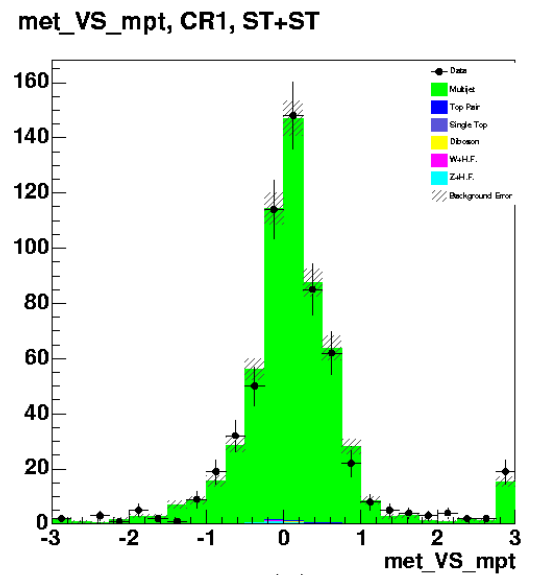

(c)

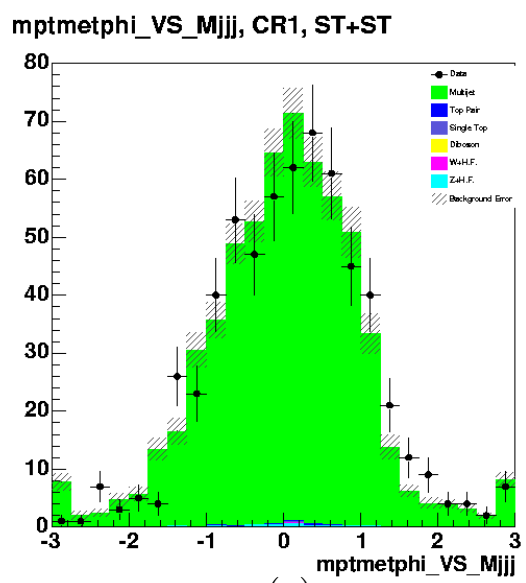

(e)
met_VS_MaxDPhiJs, CR1, ST+ST

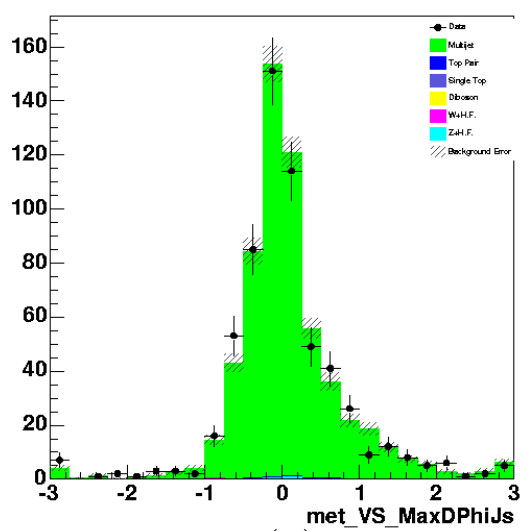

(b)

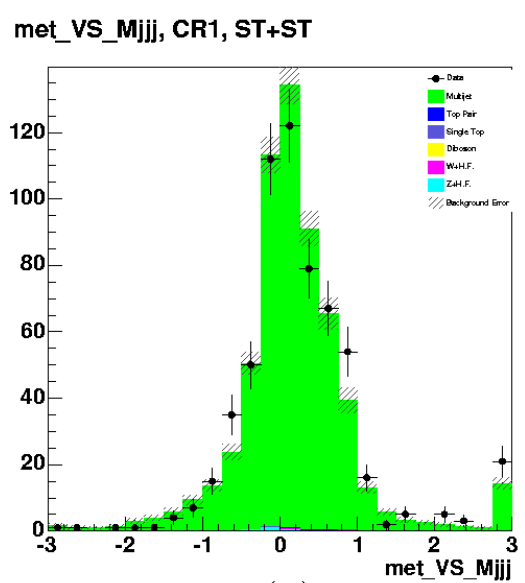

(d)

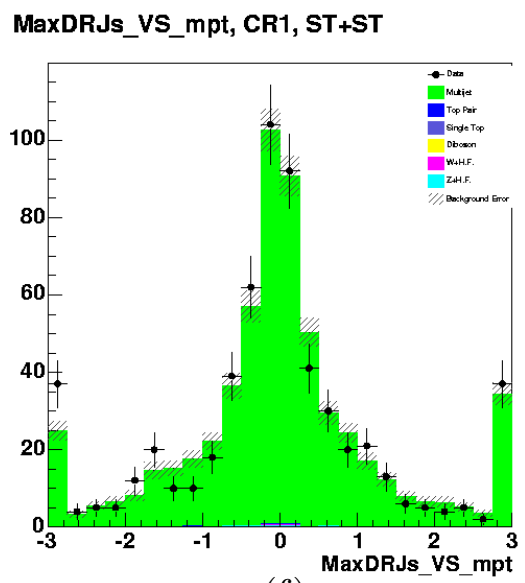

(f)

Figure C.3.: Correlations between some of the important variables in CR1, ST+ST events: (a) $r\left(\min \left(\Delta \phi\left(\overrightarrow{\mathscr{E}_{T}}, \overrightarrow{J_{i}}\right)\right), \not_{T}^{t r}\right)$,

(b) $r\left(\max \left(\Delta \phi\left(\vec{J}_{i}, \vec{J}_{k}\right)\right), \notin_{T}\right)$,

(c) $r\left(\notin_{T}, \not P_{T}^{t r}\right)$, (d) $r\left(\mathscr{E}_{T}, M_{j j}\right)$, (e) $r\left(\Delta \phi\left(\overrightarrow{\mathscr{E}_{T}}, \overrightarrow{\not_{T}^{t r}}\right), M_{j j}\right)$, (f) $r\left(\max \left(\Delta R\left(\vec{J}_{i}, \vec{J}_{k}\right)\right), \not_{T}^{t r}\right)$. 


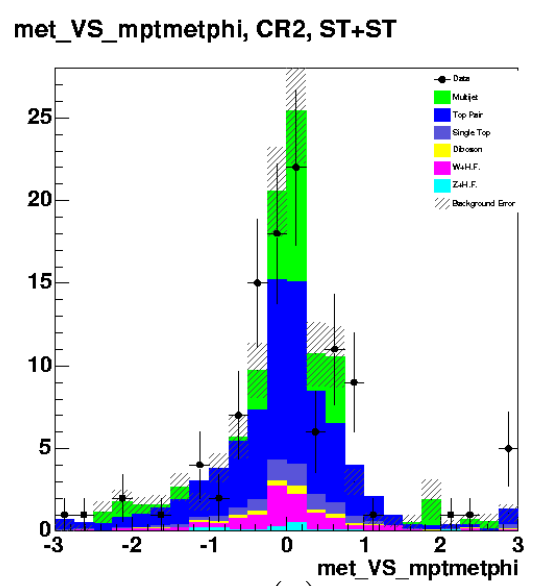

(a)

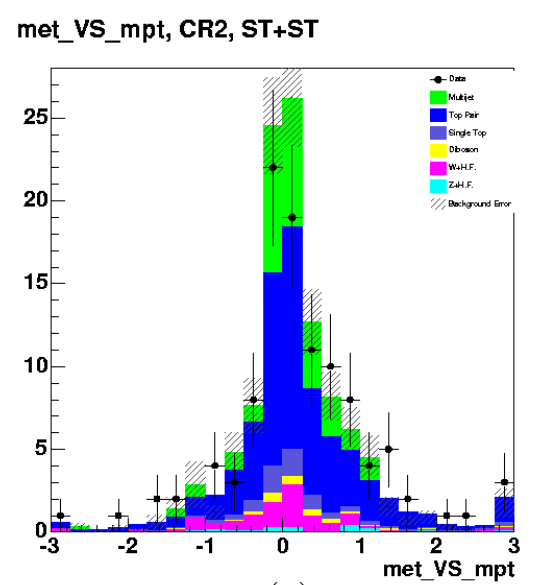

(c)

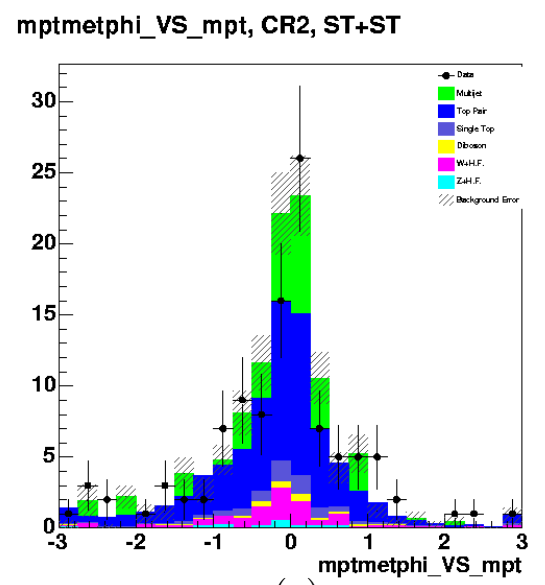

(e)
MinDPhiMET_Ji_vS_Mjjj, CR2, ST+ST

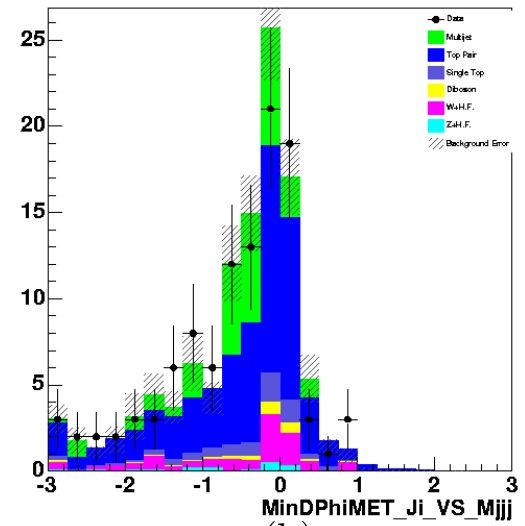

(b)

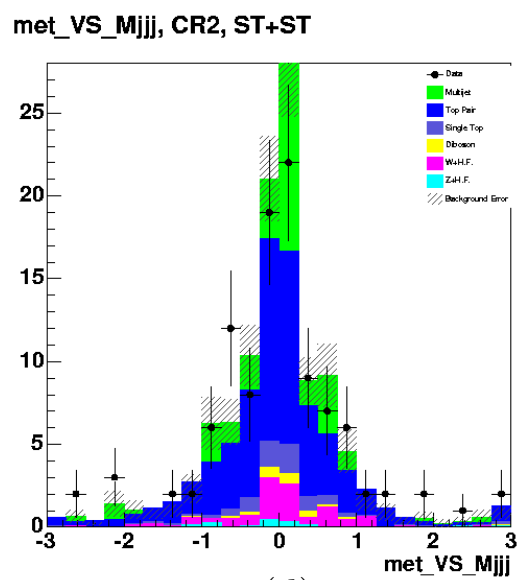

(d)

mptmetphi_VS_Mjjj, CR2, ST+ST

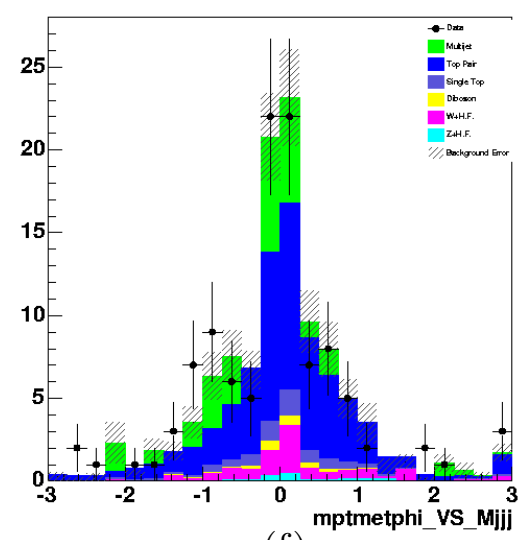

(f)

Figure C.4.: Correlations between some of the important variables in CR2,

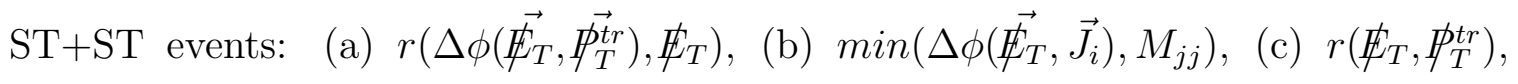
(d) $r\left(\mathscr{E}_{T}, M_{j j}\right)$, (e) $r\left(\Delta \phi\left(\overrightarrow{\mathscr{E}_{T}}, \overrightarrow{\not_{T}^{t r}}\right), \not_{T}^{t r}\right)$, (f) $r\left(\Delta \phi\left(\overrightarrow{\mathbb{E}_{T}}, \overrightarrow{\not_{T}^{t r}}\right), M_{j j}\right)$. 
MinDPhiMET_Ji_vS_mpt, CR1, ST+JP

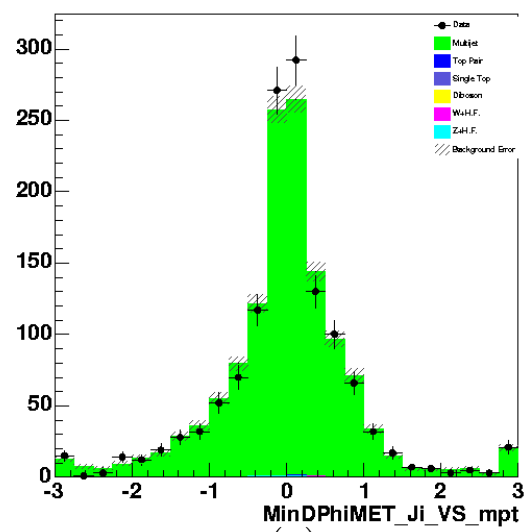

(a)

met_VS_mpt, CR1, ST+JP

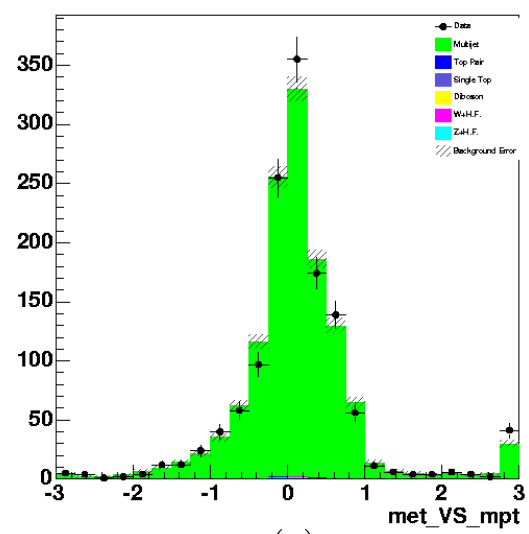

(c)

mptmetphi_VS_Mjjj, CR1, ST+JP

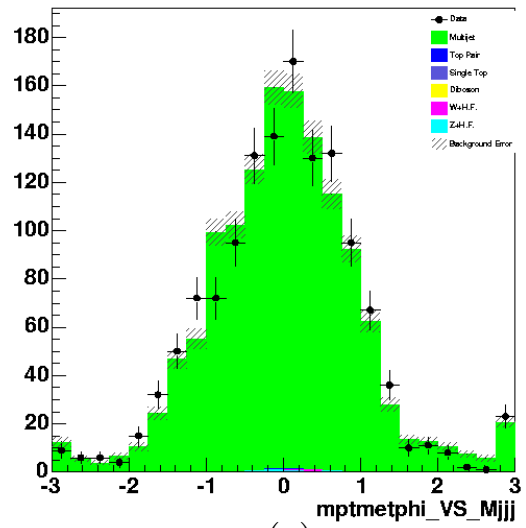

(e)
met_VS_MaxDPhiJs, CR1, ST+JP

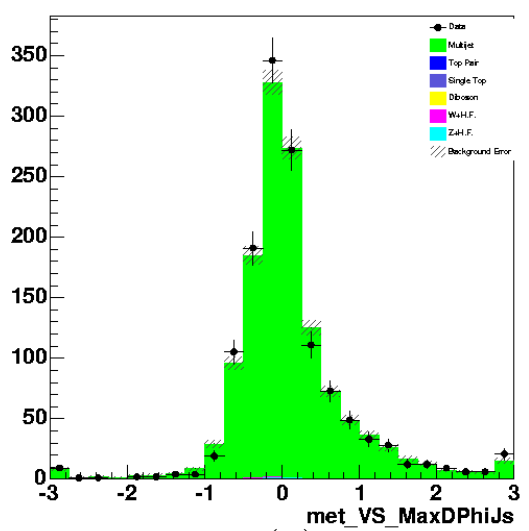

(b)

met_VS_Mjjj, CR1, ST+JP

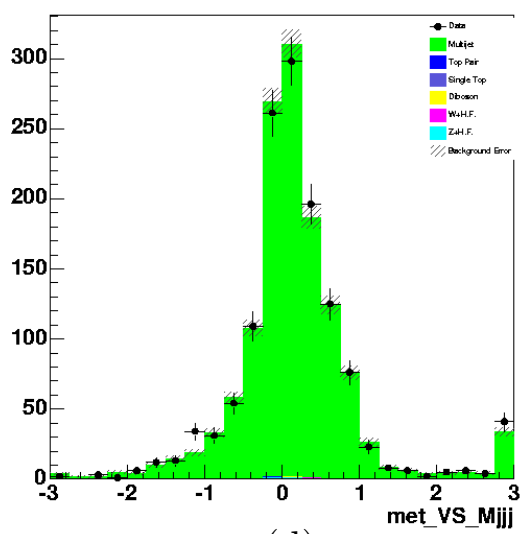

(d)

MaxDRJs_VS_mpt, CR1, ST+JP

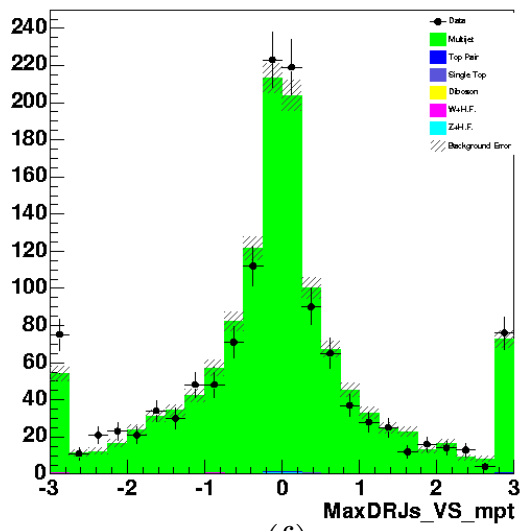

(f)

Figure C.5.: Correlations between some of the important variables in CR1, ST+JP events: (a) $r\left(\min \left(\Delta \phi\left(\overrightarrow{\mathbb{E}_{T}}, \overrightarrow{J_{i}}\right)\right), \not_{T}^{t r}\right)$,

(b) $r\left(\max \left(\Delta \phi\left(\vec{J}_{i}, \vec{J}_{k}\right)\right), \notin_{T}\right)$,

(c) $r\left(\notin_{T}, \not P_{T}^{t r}\right)$, (d) $r\left(\mathscr{E}_{T}, M_{j j}\right)$, (e) $r\left(\Delta \phi\left(\overrightarrow{\mathscr{E}_{T}}, \overrightarrow{\not_{T}^{t r}}\right), M_{j j}\right)$, (f) $r\left(\max \left(\Delta R\left(\vec{J}_{i}, \vec{J}_{k}\right)\right), \not_{T}^{t r}\right)$. 
met_VS_mptmetphi, CR2, ST+JP

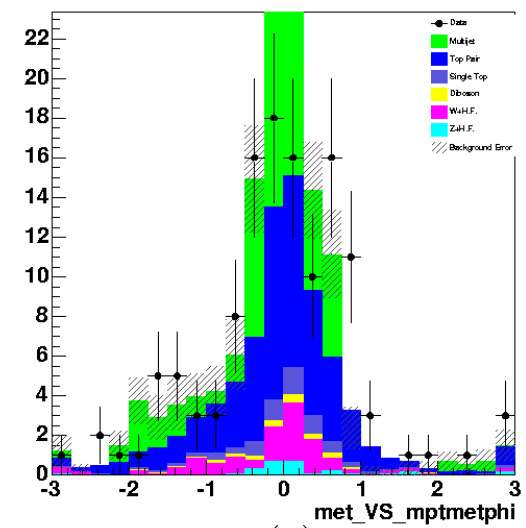

(a)

met_VS_mpt, CR2, ST+JP

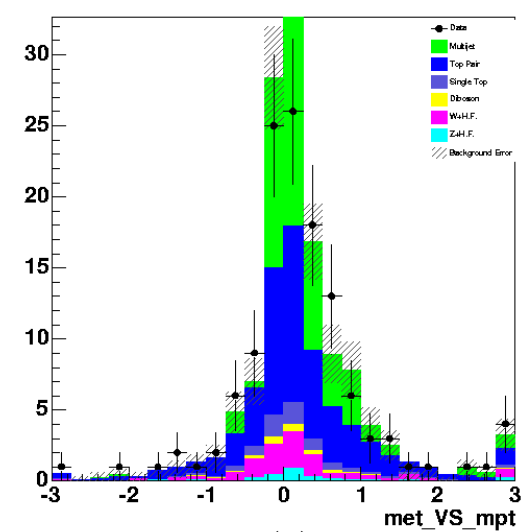

(c)

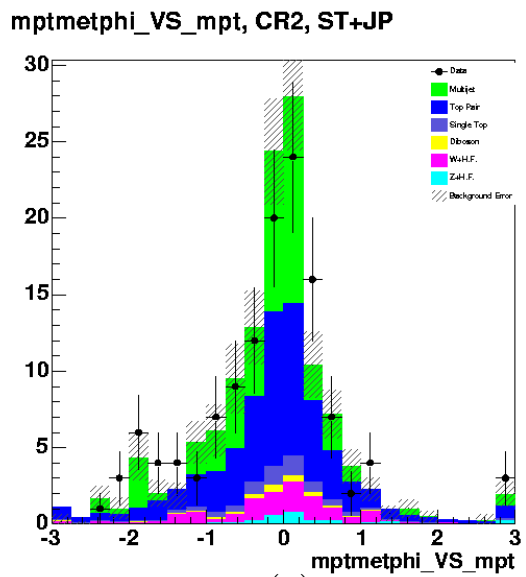

(e)
MinDPhiMET_Ji_vs_Mjij, CR2, ST+JP

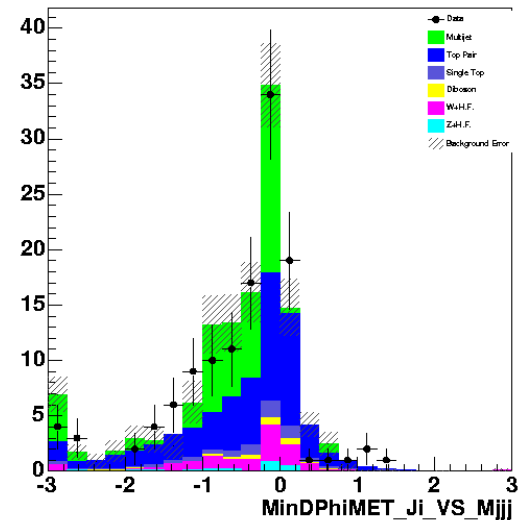

(b)

met_VS_Mjjj, CR2, ST+JP

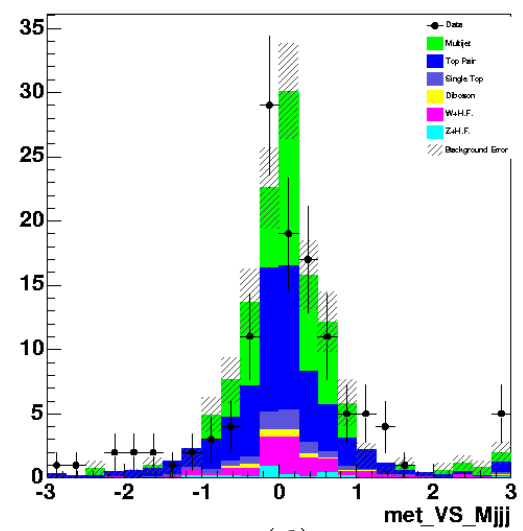

(d)

mptmetphi_VS_Mjjj, CR2, ST+JP

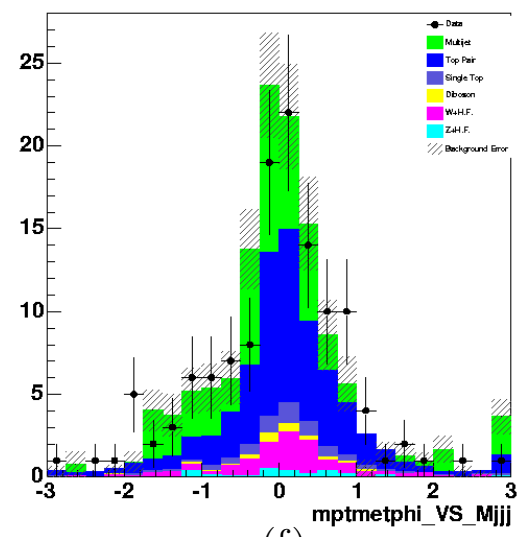

(f)

Figure C.6.: Correlations between some of the important variables in CR2, ST+JP events: (a) $r\left(\Delta \phi\left(\overrightarrow{\mathscr{E}_{T}}, \overrightarrow{\not_{T}^{t r}}\right), \notin_{T}\right), \quad(\mathrm{b}) \min \left(\Delta \phi\left(\overrightarrow{\mathscr{E}_{T}}, \overrightarrow{J_{i}}\right), M_{j j}\right), \quad$ (c) $r\left(\mathscr{E}_{T}, \not_{T}^{t r}\right)$, (d) $r\left(\mathscr{E}_{T}, M_{j j}\right)$, (e) $r\left(\Delta \phi\left(\overrightarrow{\mathscr{E}_{T}}, \overrightarrow{\not_{T}^{t r}}\right), \not_{T}^{t r}\right)$, (f) $r\left(\Delta \phi\left(\overrightarrow{\mathbb{E}_{T}}, \overrightarrow{\not_{T}^{t r}}\right), M_{j j}\right)$. 


\section{Appendix D: Additional Plots for ANNs}

As can be seen from Figure D.1, the signal significance after requiring $\mathrm{ANN}_{\mathrm{MJ}}>0$ increases by about $20 \%$. While we could have chosen to place the cut on $\mathrm{ANN}_{\mathrm{MJ}}$ at higher value, which would reduce the signal significance, it would also reduce the amount of signal events. We chose to keep more signal events and discriminate from the backgrounds at a later stage of the analysis.

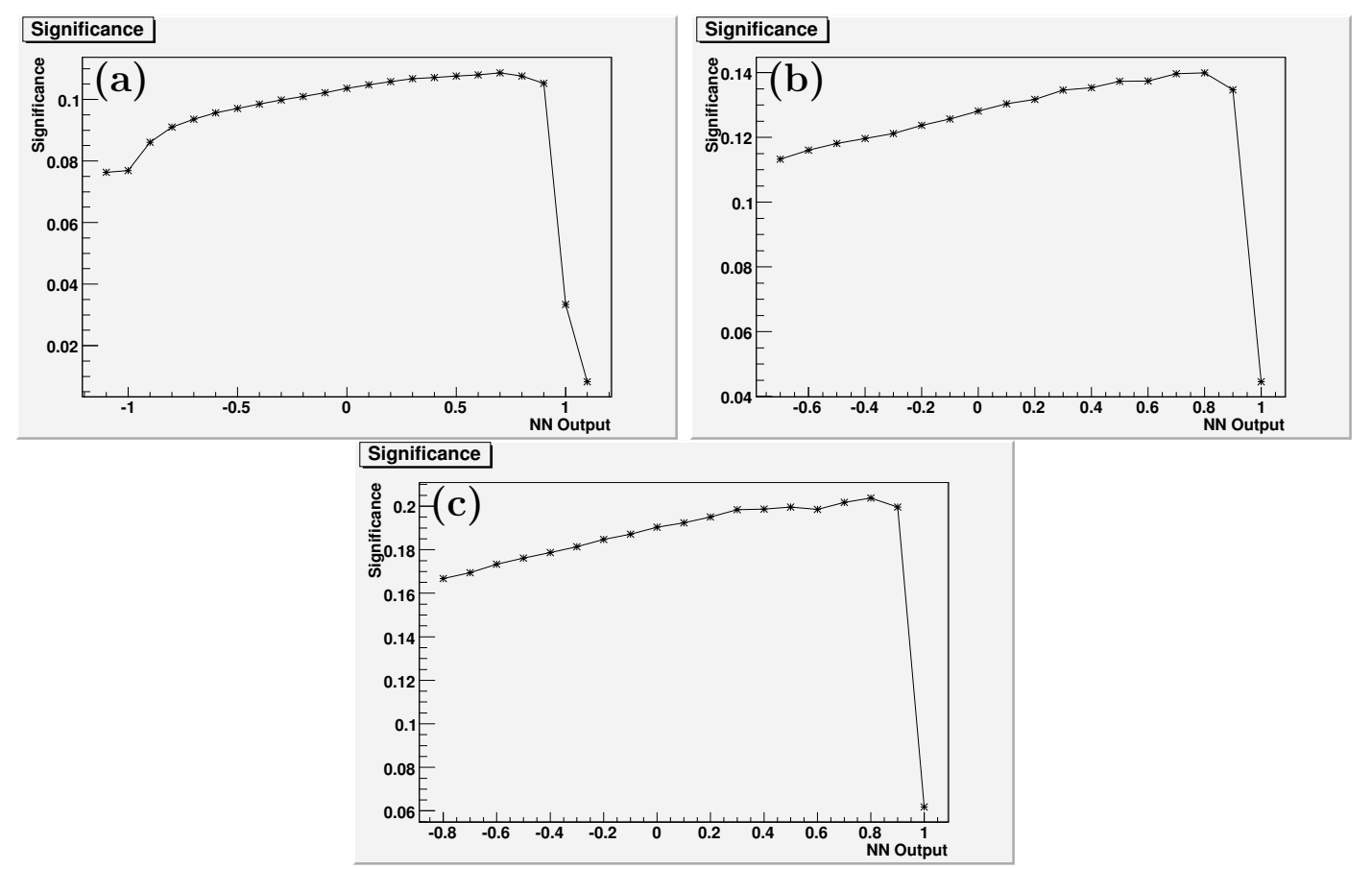

Figure D.1.: Significance of the sample as a function of the cut on $\mathrm{ANN}_{\mathrm{MJ}}$ output: (a) Exclusive ST, (b) ST+ST, (c) ST+JP.

During the ANN training, the classification algorithm utilizes the discriminating power of not only the individual kinematic variables, but also the correlations between them. This can be seen from the introduction to ANNs in Section 7.1.1. Each successive layer in an ANN is connected to the input variables through a set of weights $w_{i j}$ in Eq. 7.1. The weights of an input layer to a hidden node for correlated variables will also be correlated, and equivalent in terms of their influence on the ANN's output. Therefore, it is desirable to remove highly correlated input variables 
from the ANN, since their addition does not increase the discriminating capabilities of the classifier. Additionally, the correlation patterns that are different between background and signal samples, provide additional discriminating power, similar to kinematic variables.

The correlation patterns for the background QCD MC sample used for the training of $\mathrm{ANN}_{\mathrm{MJ}}$ are shown in Fig. D.2(a), while the ones for the signal sample are shown in Fig. D.2(b). As it can be seen from these figures, the correlation patterns are different for the two samples. The names of the variables and the order as they appear on the correlation plots is shown in Tab. D.1.

\begin{tabular}{|c|c|}
\hline Kinematic variable & Notation \\
\hline Missing transverse momentum $\not P_{T}^{t r}$ & $\mathrm{MPT}$ \\
\hline Ratio of missing $H_{T}$ and missing transverse energy $\not_{T}$ & MET \\
\hline Maximum of the difference in phi between any two jets & $\max \left(\Delta \phi\left(J_{i}, J_{k}\right)\right)$ \\
\hline Minimum of the difference in $\phi$ between $\overrightarrow{\not P_{T}^{t r}}$ and any jet & $\min \left(\Delta \phi\left(M P T, J_{i}\right)\right)$ \\
\hline Minimum of the difference in $\phi$ between $\vec{E}_{T}$ and any jet & $\min \left(\Delta \phi\left(M E T, J_{i}\right)\right)$ \\
\hline Event $H_{T}$ & $\mathrm{HT}$ \\
\hline Maximum of the difference in the $R$ space between any two jets & $\max \left(\Delta R\left(J_{i}, J_{k}\right)\right)$ \\
\hline Difference in $\phi$ between $\overrightarrow{\mathscr{E}_{T}}$ and $\overrightarrow{p_{T}^{t r}}$ & $\Delta \phi(M E T, M P T)$ \\
\hline Event $\notin_{T}$ & MET \\
\hline
\end{tabular}

Table D.1: Variables used in Fig. D.2, as they appear from top to bottom (left to right) on the correlation comparison plots. 


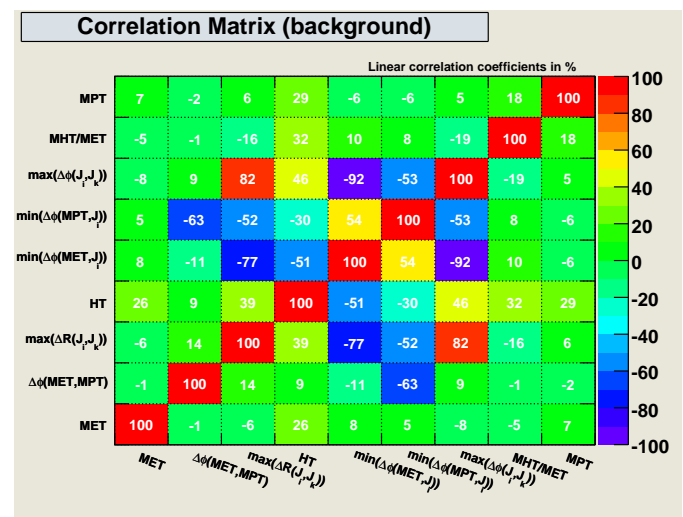

(a)

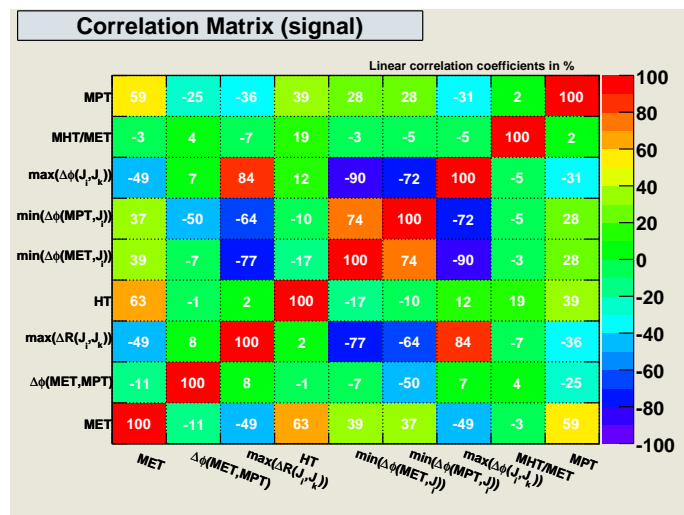

(b)

Figure D.2.: Correlations between the $\mathrm{ANN}_{\mathrm{MJ}}$ input variables, for (a) background and (b) signal samples. 
Figures D.3-D.4 show the different correlation pattern between the input variables to the $\mathrm{ANN}_{\mathrm{SIG}}$ for the signal and background training.

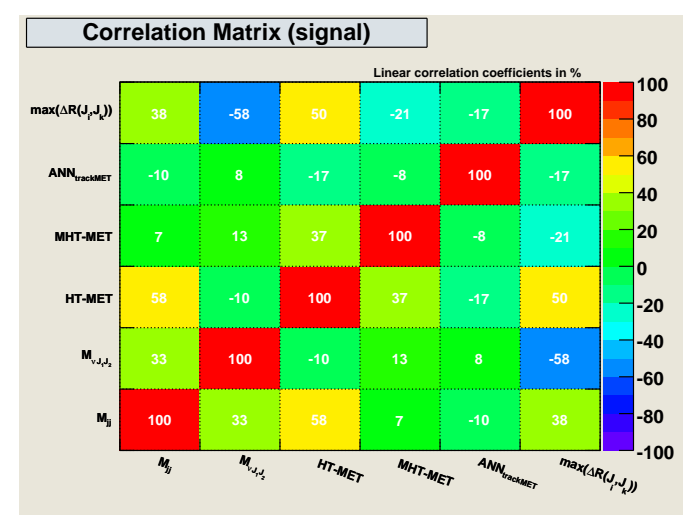

(a)

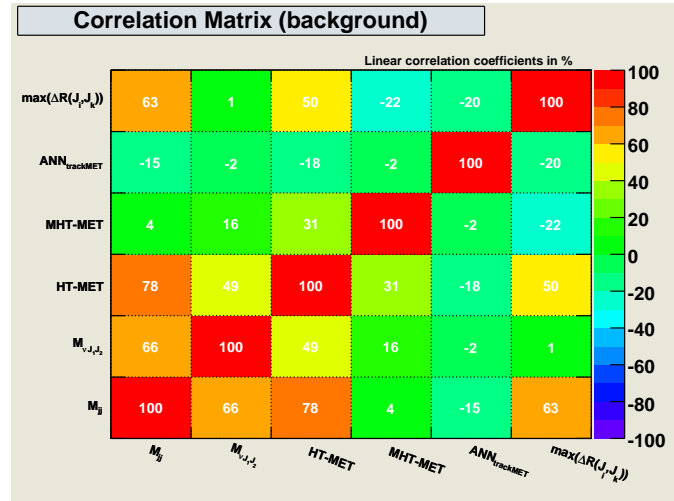

(b)

Figure D.3.: Correlations between the $\mathrm{ANN}_{\mathrm{SIG}}$ input variables for 2 jet events, for (a) background and (b) signal samples.

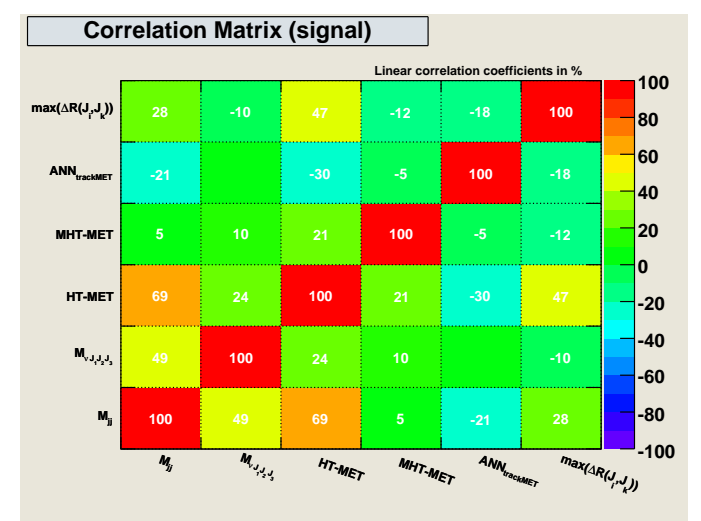

(a)

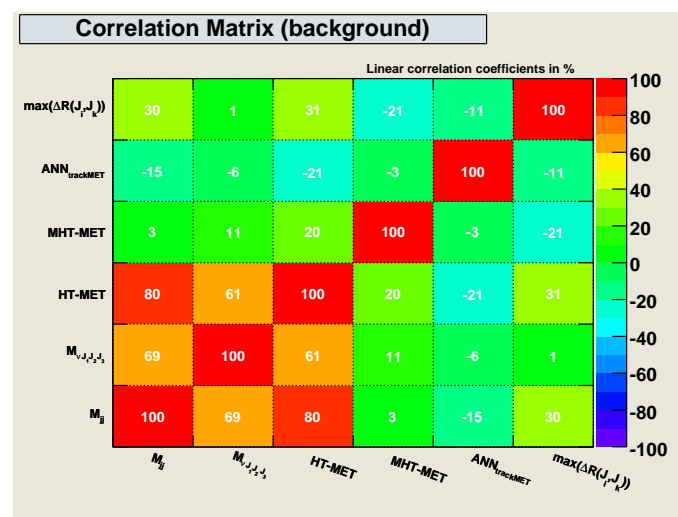

(b)

Figure D.4.: Correlations between the $\mathrm{ANN}_{\mathrm{SIG}}$ input variables for 3 jet events, for (a) background and (b) signal samples. 


\begin{tabular}{|l|c|}
\hline Kinematic variable & Notation \\
\hline \hline Maximum of the difference in the $R$ space between any two jets & $\max \left(\Delta R\left(J_{i}, J_{k}\right)\right)$ \\
Output of the TRACKMET neural network & $\mathrm{ANN}_{\text {trackMET }}$ \\
Difference between missing $H_{T}$ and missing transverse energy $\notin_{T}$ & $\mathrm{MHT}-\mathrm{MET}$ \\
Difference between $H_{T}$ and missing transverse energy $\notin_{T}$ & $\mathrm{HT}$-MET \\
Transverse invariant mass of all jets and $\notin_{T}$ & $M_{\nu, J_{1}, J_{2}, J_{3}}$ \\
Invariant mass of the two leading jets & $M_{j j}$ \\
\hline
\end{tabular}

Table D.2: Variables used in Fig. D.3-D.4, as they appear from top to bottom (left to right) on the correlation comparison plots. 
VITA 


\section{VITA}

Artur Apresyan was born in Yerevan, Armenia on July 6, 1980. He completed his high school education at No. 38 "Belinsky" school in Yerevan in 1997. He attended the Yerevan State University between Fall of 1997 and Spring 2001 where he earned his B.S. in Physics. Subsequently, he entered the graduate school at Yerevan State University to pursue the Masters degree, which he received in Spring 2003. In the Fall of 2003 he entered the graduate program at Purdue University, where he received the Ross Fellowship. He joined the CDF Collaboration at Fermilab in November 2005 as a student of Daniela Bortoletto where he conducted his thesis research until receiving his Ph.D in Physics in May 2009. 